Nenro

endocrinology

\title{
Abstracts of the
}

\section{2th Annual ENETS Conference for the Diagnosis and Treatment of Neuroendocrine Tumor Disease}

March 11-13, 2015, Barcelona, Spain

Guest Editors

Nick Reed, Glasgow

Anne Couvelard, Paris

Philippe Ruszniewski, Clichy 
Basic Science - mTOR and Other Pathways, Signalling, Receptors

Abstracts A1-A15

Basic Science - Genetics, Epigenetics, miRNAs

Abstracts B1-B9

Basic Science - In vitro Models, Tumor Growth, CTCs

Abstracts C1-C6

Epidemiology/Natural History/Prognosis - Registries,

Nationwide and Regional Surveys

Abstracts D1-D7

Epidemiology/Natural History/Prognosis - Prognosis

Abstracts E1-E12

Epidemiology/Natural History/Prognosis - Descriptive Epidemiology

Abstracts F1-F9

Pathology, Grading, Staging

Abstracts G1-G8

Biomarkers

Abstracts $\mathrm{H} 1-\mathrm{H} 16$

Imaging (Radiology, Nuclear Medicine, Endoscopy)

Abstracts I1-I19

Medical Treatment - Chemotherapy

Abstracts J1-J12

Medical Treatment - SMS Analogues, Interferon

Abstracts K1-K11

Medical Treatment - Targeted Therapies

Abstracts L1-L16

Medical Treatment - Others

Abstracts M1-M6

PRRT-Ablative Therapies-Endoscopic Treatment

Abstracts N1-N7

Surgical Treatment

Abstracts 01-016

Non Digestive NETs (Bronchial, MTC, Pheochromocytoma)

Abstracts P1-P6

Clinical Cases/Reports

Abstracts Q1-O28

... None of the Below

Abstracts R1-R11

Author Index 


\section{Basic Science - mTOR and Other Pathways, Signalling, Receptors}

\begin{abstract}
A1

\section{Abrogation of Autophagy by Chloroquine in Neuroendocrine Tumor Cells Treated with mTOR Inhibitors Induces Apoptosis, While Reduction of Cell Proliferation is Due to a Chloroquine, Autophagy Unrelated, Lysosomal Effect}

\author{
Avniel-Polak S., Leibowitz G., Glaser B., Gross D.J., \\ Grozinsky-Glasberg S. \\ Hadassah-Hebrew University Medical Center, Jerusalem, \\ Israel
}

Introduction: The therapy options for patients with advanced NETs are limited. The mTOR inhibitors (mTORi), Torin1 and NVPBEZ235, are known to suppress cell proliferation in NETs. However, cancer cells may use mTORi-induced autophagy to prolong survival, evading the anti-cancer effect. Chloroquine (CQ) and hydroxychloroquine (HCQ) have been shown to inhibit autophagy. Aim(s): To explore the mechanisms underlying the effect of CQ/HCQ and mTORi on cell proliferation, apoptosis and autophagy in a NET cell model. Materials and Methods: BON-1 cells were treated with Torin1, BEZ235, HCQ, CQ. Cell proliferation was examined by XTT and Ki67 staining. Flow cytometry and Western blot were used to assess drugs effect on cell cycle, apoptosis, PI3K/Akt/mTOR and autophagy. siRNA against ATG5 and ATG7 was used to genetically inhibit autophagy. Results: Torin 1/BEZ235 combination with HCQ/ CQ significantly reduced cell viability compared to each drug alone. Torin1 or BEZ235 combination with CQ induced a higher degree of apoptosis and accumulation of LC3-II. Since CQ is known to have pleiotropic effects on cells, we used siRNA against ATG5 and ATG7 in order to understand the mechanism underlying the inhibitory effects. Our latest results indicate that the inhibition of cell proliferation results from CQ effects on lysosomes. The induction of apoptosis, however, depends on the CQ ability to inhibit autophagy. Conclusion: CQ/HCQ potentiates the effect of mTORi, promoting apoptosis and suppressing cell proliferation in this NET cell model. Keywords: nets, autophagy, mtori.

\author{
A2 \\ p27kip1 Is Involved in the Resistance of \\ Human Bronchial Carcinoids to mTOR \\ Inhibitors \\ Benfini K., Gagliano T., Gentilin E., Falletta S., \\ Di Pasquale C., Degli Uberti E., Zatelli M.C. \\ University of Ferrara, Ferrara, Italy
}

Introduction: Bronchial carcinoids $(\mathrm{BC})$ are still orphan of medical therapy. We previously demonstrated that the typical $\mathrm{BC}$ human cell line NCI-H727 is sensitive to Everolimus (E), in terms of cell viability reduction, with a G0 cell-cycle arrest, while the atypical human BC cell line NCI-H720 is not. The mechanisms underlying this phenomenon have not been clarified. $\operatorname{Aim}(\mathbf{s})$ : Our aim is to investigate the mechanisms of resistance to mTOR inhibitors in BC cells, in order to identify new therapeutic approaches. Materials and Methods: Protein profiling of the main complexes regulating cellcycle progression (CyclinE/CDK2, CyclinD1/CDK4, CDK-inhibitor p27) was performed, before and after treatment with E. Results: $\mathrm{BC}$ cell lines showed a different basal protein profile. Basal levels of CyclinE/CDK2, CyclinD1/CDK4, p27 and pSer10 p27 are higher in the resistant $\mathrm{BC}$ cell line as compared to the sensitive one, suggesting a reduction in the inhibitory function of $\mathrm{p} 27$. After treatment with $\mathrm{E}$, a reduction in $\mathrm{CycD} 1 / \mathrm{CDK} 4, \mathrm{p} 27$ and pSer10 p27 (but not in $\mathrm{CycE} / \mathrm{CDK} 2$ ) protein levels is observed in the resistant cell line despite the cells continue to proliferate. On the contrary, in the human $\mathrm{BC}$ sensitive cell line, E reduces CycD1 levels, without influencing the other cell cycle proteins, suggesting that other mechanisms are involved in G0 arrest in this cell line. Conclusion: Our data indicate that p27 is probably involved in the resistance to mTOR inhibitors in BC cells which escape G0 arrest, likely due to an impaired p27 function. Keywords: rad001, bc, p27kip1, cell cycle.

\section{A3 \\ mTOR Down-Stream Signaling Pattern May Predict pNET Response to Everolimus \\ Falletta S. , Gagliano T. , Gentilin E. a Di Pasquale C. ${ }^{\text {, }}$ Benfini K. ${ }^{a}$, Polenta V. ${ }^{b}$, Falconi M. ${ }^{\text {, }}$ Partelli S. ${ }^{\text {, }}$ Degli Uberti E. a Zatelli M.C. ${ }^{a}$ \\ aUniversity of Ferrara, Ferrara, Italy; bUniversity of Marche Region, Ancona, Italy}

Introduction: NETs are rare neoplasms arising from neuroendocrine cells spread in the respiratory and gastro-entero-pancreatic epithelium. The mammalian target of rapamycin (mTOR) pathway plays a central role in the regulation of cell growth, metabolism, and 
apoptosis representing a novel molecular target. This pathway is constitutively activated in pancreatic NETs (pNETs) providing the basis for the development of specific mTOR inhibitors as new therapeutic tools. Aim(s): To investigate the effect of Eve, a mTOR inhibitor, on cell viability and apoptosis activation in pNETs and identify possible markers of the efficacy of mTOR inhibitors that may help in identifying the patients who may benefit from treatment with mTOR inhibitors. Materials and Methods: 20 pNET primary cultures were treated with IGF1 and/or Eve. Cell viability and caspase activity were evaluated. Alpha Screen Assays for PI3K/AKT/mTOR pathway components were performed. Results: In 6 pNET Eve reduced cell viability by $30 \%$ with a concomitant increase in apoptosis. Eve blocked IGF-1 proliferative and anti-apoptotic effects. In 14 pNET Eve and IGF-1 did not affect cell viability and apoptosis. IGF1R, AKT, mTOR and 4EBP1 phosphorylation was significantly $(\mathrm{p}<$ 0.001 ) higher in the Responder as compared to the Non Responder group. Conclusion: Eve reduced cell viability in a sub-group of pNETs. The data suggest that IGF1R and mTOR down-stream signaling is hampered in 'Non Responder' pNETs, possibly predicting treatment outcome. Keywords: pnet, everolimus, mtor.

\section{A4 \\ Role of TGF Beta-1 in Regulating Pancreatic Neuroendocrine Tumor Cell Viability}

\section{Falletta S. a, Gagliano T. , Gentilin E. a, Di Pasquale C. , Benfini K. ${ }^{a}$, Polenta V. ${ }^{\mathrm{b}}$, Falconi M. ${ }^{\mathrm{b}}$, Partelli S. ${ }^{\mathrm{b}}$, Degli Uberti E. ${ }^{\text {, }}$ Zatelli M.C. ${ }^{\text {a }}$

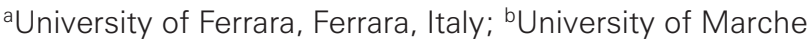 Region, Ancona, Italy}

Introduction: Neuroendocrine tumors (NETs) are heterogeneous neoplasms arising from neuroendocrine cells spread in the respiratory and gastro-entero-pancreatic epithelium. The role of transforming growth factor beta-1 (TGF b-1) in NET biology is largely unknown. TGF b-1 signaling pathway is tumor suppressive in most non-transformed epithelial cell lines. In contrast, many human carcinomas are refractory to the growth-inhibitory effects of TGF b-1. Aim(s): To investigate if TGF b-1 may modulate cell viability and apoptosis in NET of the pancreas (pNET) and to understand whether TGF b-1 may influence the effects of therapeutic molecules currently used in the management of pNETs. Materials and Methods: 20 primary cultures obtained from surgical samples of pNETs were treated with TGF b-1 and/or Everolimus, a mTOR inhibitor. Cell viability and caspase activity were evaluated. Results: In 4 pNETs TGF b- 1 reduced cell viability by $50 \%$ with a concomitant increase in apoptosis. In 16 pNETs TGF b-1 induced an increase in cell viability by $30 \%$ with a concomitant decrease in apoptosis. In the first group, the combination of TGF b-1 and Everolimus induced a significant decrease in cell viability by $65 \%$, which was not observed under treatment with Everolimus alone. Conclusion: In conclusion, TGF b-1 reduces cell viability of a pNET sub-group. In addition, TGF beta-1 may cooperate with Everolimus in inducing growth arrest. Further studies are necessary to understand TGF b-1 related functional context in PCC. Keywords: pnet, tgf-b1, everolimus.

\section{A5}

\section{Dual Inhibition of PI3K and mTORC1/ \\ C2 by PKI-587 (PF-05212384) as a \\ Promising Therapeutic Option for Gastroenteropancreatic Neuroendocrine Tumor Disease and Its Effect on AKT-Signaling}

\author{
Freitag H., Briest F., Christen F., Grass I., Lewens F., \\ Siegmund B., Grabowski P. \\ Charité, Berlin, Germany
}

Introduction: Gastroenteropancreatic neuroendocrine neoplasms (GEP-NEN) are heterogeneous in their clinical behavior and therapeutic options are still not satisfactory. The 'crosstalk' of different signaling pathways in NEN cells appears to be more complex as known already. PKI-587 is a highly potent novel dual inhibitor of PI3K and mTORC1/C2. Aim(s): Therefore, we assessed the effects of PKI-587 in different GEP-NEN cell lines compared to the established mTORC1 inhibitor RAD001. Materials and Methods: We treated the cell lines BON, QGP-1, KRJ-1 and LCC-18 with increasing concentrations of the inhibitor PKI-587, compared to RAD001 and DMSO. We performed WST-1 assay to determine efficacy and growth inhibition. The induction of apoptosis was shown by caspase $3 / 7$ activation and cell cycle was analyzed by FACS. We determined alterations of signaling mediators by phosphor-specific Western Blot analysis. Results: In all cell lines PKI-587 inhibited dose-dependently proliferation with an IC50 of 25-250 nM, whereas RAD001 was less effective. Treatment with PKI-587 led to cell cycle arrest and induction of apoptosis. Protein analysis showed decreased mTORC2 and PI3K mediated activation of AKT. Moreover, the reduction of activity of direct mTORC1 targets 4E-BP and S6K by dual inhibition was much stronger than by sole mTORC1 inhibition. Conclusion: $\mathrm{PI} 3 \mathrm{~K} / \mathrm{mTOR}$ dual targeting is a promising new therapeutic approach in neuroendocrine tumor disease, what should be evaluated in further clinical trials. Keywords: dual inhibition, pi3k, mtor, pki-587, rad001, signaling.

\section{A6}

Receptor Tyrosine-Kinases Inhibition: New Insights on Therapeutic Implications of EGFR and IGF1R in Human Bronchopulmonary NET

\author{
Gagliano T., Benfini K., Gentilin E., Falletta S., \\ Di Pasquale C., Caneva E., Riva E., Balboni F., \\ Degli Uberti E., Zatelli M.C. \\ University of Ferrara, Ferrara, Italy
}

Introduction: The main treatment for bronchopulmonary NET (BP-NET) is surgery, which is not feasible for infiltrating and metastatic disease, where medical therapy is tried. Identifying new therapeutic targets is important to provide adequate medical treatment for patients with BP-NET. Sunitinib, a multi-targeted receptor tyrosine kinase inhibitor (TKI), is mainly described to inhibit VEGF. $\operatorname{Aim}(\mathbf{s})$ : To verify if IGF1R and EGFR could be involved in Sunitinib 
mechanism of action in BP-NET. Materials and Methods: Human BP-NET cell lines and human BP primary cultures were treated with sunitinib and/or EGF, IGF1, or VEGF. Cell viability and caspase 3/7 activation were measured after $48 \mathrm{~h}$ of treatment. Receptor expression was detected by Western blot in cell lines and primary cultures. IGF1R and EGFR phosphorylation was analysed by alphascreen sure fire assay. Results: Sunitinib inhibits cell viability (by 50\%) and activates caspase $3 / 7$ (by 100\%) in BC cell lines and primary cultures. Both events are counteracted by EGF and IGF-1, but not by VEGF. Cell lines and tissues express IGF1R and EGFR, while VEGFR are not expressed in all samples. Treatment with Sunitinib decreased the phosphorylation of both EGFR and IGFR1, in both cell lines and primary cultures. Conclusion: These data indicate that EGFR and IGF1R expression is important for Sunitinib activity in BP-NET. The effects of Sunitinib on BP-NET cell viability could be a result of a double inhibition of EGFR and IGF1R. (208). Keywords: rtk, bpnet, igflr, egfr.

\section{A7 \\ Role of Amplifications in the PIK3/Akt/ mTOR-Pathway in Neuroendocrine Tumors of the Small Intestine}

Hoffmeister M. ${ }^{a}$, Sperveslage J. ${ }^{\mathrm{b}}$, Anlauf M. ${ }^{c}$, Petersen K. ${ }^{a}$, Gress T. ${ }^{\mathrm{d}}$, Moll R. ${ }^{\mathrm{e}}$, Arsenic R. ${ }^{\mathrm{f}}$, Pape U.F. ${ }^{\mathrm{g}}$ Sipos B. ${ }^{\mathrm{a}}$

alnstitute for Pathology and Neuropathology, University Hospital of Tübingen, Tübingen, Germany; ${ }^{\text {b Gerhard- }}$ Domagk-Institute for Pathology, University Hospital of Münster, Münster, Germany; 'Institute for Pathology and Cytology, St. Vincenz Hospital Limburg, Limburg, Germany; dDepartment of Gastroenterology, Endocrinology and Metabolism, Philipps-University Marburg, Marburg, Germany; 'Department of Pathology, Philipps-University Marburg, Marburg, Germany; fInstitute for Pathology, Charité University Medicine, Berlin, Germany; ' Department of Hepatology and Gastroenterology, Charité University Medicine, Berlin, Germany

Introduction: Amplifications in the PIK3/Akt/mTOR pathway can lead to increased proliferation and reduced apoptosis, resulting in the development of various cancers. Recently, amplifications of Akt1/2, PDGFR $\alpha / \beta$ and mTOR in small intestine neuroendocrine tumors (siNET) have been shown. Aim(s): We sought to identify the distribution and biological relevance of amplifications of Akt1/2, PDGFR $\alpha / \beta$ and mTOR in small intestine neuroendocrine tumors analyzing a large cohort. Materials and Methods: Gene amplifications were assessed by Fluorescence-in-situ-Hybridization (FISH). Tissue microarrays consisting of 217 siNET samples of 135 different patients were used. Amplification was determined by the ratio of Akt1/2, PDGFR $\alpha / \beta$, mTOR specific signals and of reference chromosome signals. Samples showing $>2$ signals in $>30 \%$ of cells were referred to as amplified. Results: $49 \%$ of the patients (56/115) had amplifications in the mTOR pathway. Significantly more amplifications $(\mathrm{p}=0.001)$ were detected when comparing more progressed tumor stages $\mathrm{T} 3+\mathrm{T} 4(64 \%, 49 / 77)$ with earlier tumor stages $\mathrm{T} 1+\mathrm{T} 2$
(29\%, 10/34). Similarly, UICC stage IIIB tumors $(35 \%, 13 / 37)$ had significantly less amplifications than stage IV tumors (66\%, 35/53; $\mathrm{p}=0.0052$ ). Patients with advanced siNET (IIIB+IV) showed significantly shorter overall survival when having an amplification of the mTOR gene compared to patients with no amplification $(p=0.0251)$. Conclusion: Amplifications in the mTOR pathway are associated with more advanced tumor stages and adverse prognosis. Keywords: net, gene amplification, mtor pathway.

\section{A8 \\ Co-Expression of Somatostatin and CXCR4 Receptors as Targets for Diagnostics and Treatment in Intestinal Neuroendocrine Neoplasms \\ Kaemmerer D. ${ }^{a}$, Träger T. ${ }^{\mathrm{b}}$, Hoffmeister M. ${ }^{\mathrm{c}}$, Sipos B. ${ }^{\mathrm{c}}$, Hommann M. ${ }^{a}$, Sänger J. ${ }^{d}$, Schulz S. ${ }^{b}$, Lupp A. ${ }^{b}$ \\ aZentralklinik Bad Berka, Bad Berka, Germany; ' University Hospital Jena, Jena, Germany; 'University Hospital Tübingen, Tübingen, Germany; 'Laboratory of Pathology and Cytology Bad Berka, Bad Berka, Germany}

Introduction: Somatostatin receptors (SSTR) are widely distributed in well-differentiated neuroendocrine tumors (NET) and serve as primary targets for diagnostics and treatment. An overexpression of the chemokine receptor CXCR4, in contrast, is considered to be present mainly in highly proliferative and advanced carcinomas. Aim(s): Comparative data are still lacking, however, for neuroendocrine carcinomas (NEC). Materials and Methods: SSTR subtypes (1, 2A, $3,5)$ and CXCR4 expression was evaluated in G1 $(n=31), G 2(n=$ 47) and low (G3a; Ki-67: 21-49\%) and highly proliferative (G3b; Ki-67: $>50 \%)$ G3 $(n=43)$ neuroendocrine neoplasms by means of immunohistochemistry using novel monoclonal rabbit anti-human anti-SSTR and anti-CXCR4 antibodies and correlated to clinical data. Results: Both CXCR4 and SSTR were widely expressed in all intestinal neuroendocrine neoplasms. The CXCR4 expression differed significantly between G1 and G3; and even between G3a and G3b specimens. CXCR4 expression was directly correlated to Ki-67 (rs: $0.39 ; \mathrm{p}<0.001)$. SSTR2A exhibited an inverse association to Ki-67 (rs: $-0.5 ; \mathrm{p}<0.001$ ) and was highly expressed in G1 and G2 tumors, whereas G3 carcinomas displayed a significantly reduced expression. Conclusion: For the first time a co-expression of the SSTR-subtypes and CXCR4 were investigated. With increasing malignancy an elevation of the CXCR4 but a decrease of the SSTR2A expression was seen. The high CXCR4 expression in G3 carcinomas recommends the CXCR4 as a new target target for diagnostics and treatment in this entity. Keywords: sstr. 
A9

\section{Significance of ATRX/DAXX Expression and Alternative Lengthening of Telomeres in Insulinomas and Neuroendocrine Tumors of Small Intestine}

\author{
Neumayer B. ${ }^{a}$, Normand L. ${ }^{b}$, Hoffmeister M. ${ }^{a}$, Friemel J. ${ }^{c}$, \\ Perren A. ${ }^{\mathrm{b}}$, Gress $T^{\mathrm{d}}{ }^{\mathrm{d}}$, Sipos $B{ }^{\mathrm{a}}$ \\ anstitute for Pathology and Neuropathology, University

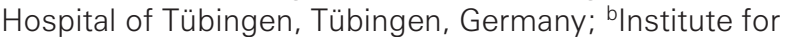 \\ Pathology, University Hospital of Bern, Bern, Schweiz; \\ Institute for Pathology, Klinikum Bremen Mitte, Bremen, \\ Germany; dDepartment of Gastroenterology, \\ Philipps-University Marburg, Marburg, Germany
}

Introduction: ATRX and DAXX mutations associated with alternative lengthening of telomeres (ALT) phenotype have been shown as one of the major altered pathways in pancreatic neuroendocrine tumors (PanNET). Aim(s): We investigated the relevance of these alterations in a large set of insulinomas and small intestine neuroendocrine tumors (siNET). Materials and Methods: In our study we characterized 78 insulinomas (65 with benign and 13 with malignant behaviour) and 137 siNET ( 54 with benign and 84 with malignant behaviour). We examined the loss of expression of DAXX and ATRX by immunohistochemistry. Further we analysed them for ultrabright signals by telomere specific fluorescence in situ hybridisation as an evidence for an ALT phenotype. Results: We detected a loss of expression of DAXX or ATRX in 6 of 78 insulinomas. 4 insulinomas showed an ALT phenotype, all with evidence of metastasis. One case of 137 siNET had a loss of expression of DAXX, which was negative for ALT. We classified 2 siNET as ALT positive, none with loss of DAXX expression. Conclusion: DAXX and ARTX mutations are correlated with the ALT pathway in insulinoma and occurred in malignant cases only. Patients with ALT positive malignant insulinomas were larger ( $3 \mathrm{~cm}$, StDev 2.61) than malignant insulinomas without ALT phenotype ( $1.35 \mathrm{~cm}$, StDev 0.5). In siNET the ALT phenotype and loss of ARTX and DAXX expression play a minor role. Keywords: daxx, atrx, alternative lengthening of telomeres, neuroendocrine tumor, insulinoma.

\section{A10 \\ mTORC1 Complex Is Significantly Over-Activated in SDHx-Mutated Paragangliomas}

Oudijk L. ${ }^{a}$, Papathomas T. ${ }^{\mathrm{a}}$, De Krijger R. Gimenez-Roqueplo A.P. , Mannelli M. , Rapa I. e, Robledo M. ${ }^{f}$ Smid M. ${ }^{\text {, }}$ Papotti M. ${ }^{\mathrm{e}}$, Volante M. ${ }^{\mathrm{e}}$

aDepartment of Pathology, Erasmus MC-University Medical Center, Rotterdam, The Netherlands;

bDepartment of Pathology, Reinier de Graaf Hospital, Delft, The Netherlands; 'Université Paris Descartes, Sorbonne, Paris, France; dDepartment of Clinical Physiopathology, University of Florence, Florence, Italy; eUniversity of Turin, Department of Oncology, Orbassano, Turin, Italy; fSpanish National Cancer Research Centre (CNIO) and ISCIII Center for Biomedical Research on Rare Diseases (CIBERER), Madrid, Spain; ' Department of Medical Oncology, Erasmus MC-University Medical Center, Rotterdam, The Netherlands

Introduction: The activation patterns of mTOR pathway in sporadic and hereditary pheochromocytomas (PCC) and paragangliomas (PGL) are poorly recognized. Aim(s): To test mTOR pathway-related molecules in a series of 178 PCC and 44 PGL, already characterized for the presence of germline mutations in VHL, RET, NF-1, MAX, SDHA, SDHB, SDHC, SDHD and somatic mutations in VHL, RET, HRAS and MAX. Materials and Methods: Cases were included into five TMAs and tested using immunohistochemistry for mTOR and Rictor, and the phosphorylated forms of mTOR, p70S6K, AMPK, AKT, 4E-BP1, S6 and Raptor. Results: Total mTOR, p-S6K and p-S6 and mTORC1-associated molecules p-Raptor and p-AMPK were all significantly over-expressed in PGLs rather than in PCCs, and in head and neck-located as compared with abdominal PGLs. None of the markers, except the low expression of p-mTOR, was associated to malignancy. Concerning genotype-to-phenotype correlations, Cluster 1 had higher total mTOR, p-Raptor and p-S6 expression than Cluster 2 PCC/PGL. In contrast, p-mTOR and mTORC2-associated molecule Rictor were significantly over-expressed in Cluster 2 tumors. Within Cluster 1, molecules active in the mTORC1 complex were significantly over-expressed in SDHx- as compared to VHLmutated tumors. Conclusion: The mTOR pathway is activated in a high proportion of PCC/PGLs, with a preferential over-activation of mTORC1 complex in PGLs of the head and neck and/or harbouring SDHx mutations. Keywords: pcc, pgl, mtor, sdhx. 
A11

\section{Platelet Serotonin But Not Dopamine Concentrations Are Lower in Pancreatic Neuroendocrine Tumor and Renal Cell Carcinoma Patients Compared to Healthy Individuals}

Peters M., Van Faassen M., Van Asselt S., Bouma G., Meijer C., Walenkamp A., De Vries E., Kema I., Oosting S.

University Medical Center Groningen, Groningen, The Netherlands

Introduction: Platelets carry VEGF-A, serotonin and dopamine. Serotonin stimulates and dopamine inhibits tumor angiogenesis. We hypothesized that platelets are activated in tumor vasculature and release angiogenic factors, resulting in lower platelet serotonin and dopamine. Aim(s): To compare platelet biogenic amine levels in pancreatic neuroendocrine tumor (pNET) and renal cell carcinoma (RCC) patients with controls and to explore a possible correlation between platelet biogenic amine and VEGF-A levels. Materials and Methods: In a cross-sectional case control study, serotonin and dopamine levels were analyzed in platelet rich and platelet poor plasma of metastatic pNET and RCC patients and controls using high performance liquid chromatography-tandem mass spectrometry. Platelet VEGF-A was analyzed with ELISA. Results: Compared to controls, platelet serotonin level was lower in 20 pNET patients (median $283 \mathrm{ng} / \mathrm{mL}$, range $135-596 \mathrm{ng} / \mathrm{mL}$ versus $162 \mathrm{ng} / \mathrm{mL}$, 45.1-703 ng/mL, $\mathrm{P}=0.009$ ) and $20 \mathrm{RCC}$ patients (median $266 \mathrm{ng} /$ $\mathrm{mL}$, range $80.9-703 \mathrm{ng} / \mathrm{mL}$ versus $159 \mathrm{ng} / \mathrm{mL}, 27.3-336 \mathrm{ng} / \mathrm{mL}, \mathrm{P}<$ 0.001). Platelet dopamine level was similar in patients and controls. Platelet biogenic amine levels did not correlate with VEGF-A levels. Conclusion: Platelets of metastatic pNET and RCC patients contain lower serotonin but similar dopamine levels compared with controls. Future studies are warranted to evaluate possible causes and consequences of low platelet serotonin levels in pNET and RCC patients. Keywords: serotonin, dopamine, pancreatic neuroendocrine tumor, renal cell carcinoma.

\section{A12 \\ Expression and Role of the CXCR4/CXCL12/ CXCR7 Axis and Crosstalk with the mTOR Pathway in Neuroendocrine Tumors (NETs)}

\author{
Sciammarella C. ${ }^{\text {, }}$ Circelli L. b, Guadagno E. a, Tafuto S. ${ }^{\text {, }}$ \\ Del Basso De Caro M.L. a, Pezzullo L. b, Marotta V.a, \\ Ramundo V. ${ }^{\mathrm{a}}$, Tatangelo F. ${ }^{\mathrm{b}}$, Losito N. ${ }^{\mathrm{b}}$, Izzo F. ${ }^{\mathrm{b}}$, Colao A. ${ }^{\mathrm{a}}$, \\ Scala S. ${ }^{\text {, }}$ Faggiano A. $^{\text {a }}$ \\ aUniversity of Naples 'Federico II', Naples, Italy; ' $N a t i o n a l$ \\ Cancer Institute, 'Fondazione G. Pascale', Naples, Italy
}

Introduction: Chemokine receptor CXCR4 has been shown to signal on mTOR pathway in gastric and renal cancer. CXCR4 interacts with the chemokine CXCL12 to exert proliferative and chemotactic effects. Aim(s): To evaluate expression and prognostic role of the CXCR4-CXCL12-CXCR7 axis and its relationship with the mTor pathway in NET. Materials and Methods: 61 human NETs were included: 40 GEP-NETs and 21 MTC were analyzed for expression of CXCR4/CXCL12/CXCR7 and mTOR pathways by immunohistochemistry (IHC). CXCR4/CXCR7 was evaluated on NCIH727 and BON cell lines by qRT-PCR and Western-Blot. The effect of mTOR and CXCR4 inhibitors (RAD001, AMD3100) was assessed by MTT assay. Results: The IHC score of CXCR4, mTOR, p-mTOR, p-4EBP1, p-S6K1 were significantly higher in G1/ G2 tumors. CXCR4, CXCR7, mTOR, p-mTOR and p-4EBP1 significantly correlated with poor prognosis, while CXCL12 $(\mathrm{p}<0.01)$ with favorable prognosis. In BON cells, CXCL12 increased pP70S6K and p4EBP1 and AMD3100 inhibited this induction. CXCL12 induced pERK 1/2, p-P38 and pAkt, while AMD3100 inhibited CXCL12induced. RAD001 modestly impaired CXCL12-mediated pERK1/2, p-P38 and pAkt induction. In both cell lines, RAD001 and AMD3100 reduced cell viability. The addition of AMD3100 $(\mathrm{P}<0.05)$ improved RAD001 anticancer activity. Conclusion: CXCR4/CXCL12/ CXCR7 axis and mTOR pathway may be reliable prognostic indicators in NET. Moreover the concomitant inhibition of CXCR4 and mTOR significantly decreased human NET cell growth compared to single drug treatment. Keywords: chemokine, mtor, nets, clinical outcome.

\section{A13 \\ mTOR Pathway Inhibition Sensitizes \\ Insulinoma Cells to Streptozotocin Induced Apoptosis}

\begin{abstract}
Vercherat C. ${ }^{a}$, Walter T. ${ }^{\mathrm{b}}$, Massoma-Peh P., Bollard J. ${ }^{\mathrm{a}}$
Lacheretz-Bernigaud A. ${ }^{a}$, Colombé M. ${ }^{a}$, Goddard I. ${ }^{\text {, }}$

Gadot N. ${ }^{a}$, Hervieu V. ${ }^{\mathrm{b}}$, Scoazec J.Y. ${ }^{\mathrm{c}}$, Cordier-Bussat M. ${ }^{\mathrm{a}}$,

Roche $\mathrm{C}^{\text {a }}$

${ }^{a}$ CRCL U1052, Lyon, France; ${ }^{b}$ Hospices Civils de Lyon,

Lyon, France; 'Institut Gustave Roussy, Villejuif, France
\end{abstract}

Introduction: Pancreatic neuroendocrine tumors (pNETs) are generally chemoresistants probably due to low proliferation rate and defects in apoptotic pathway. Targeted therapies are new encouraging options for pNETs however; clinical trials show limited objective response. Combination of chemotherapy and targeted therapy could be a new solution in therapeutic care. Streptozotocin (STZ) is the 1 st line of therapy for unresectable pNETs. $\operatorname{Aim}(\mathbf{s})$ : Based on the literature, we hypothesized that mTOR pathway over-activation could lead to resistance to STZ. Materials and Methods: To evaluate this, we combined mTOR pathway inhibitors to STZ in in vitro and in vivo models. 4 mTOR pathway inhibitors (Everolimus, MK2206, BKM120 and BEZ235) were tested. Cell viability, proliferation and apoptosis were assessed in INS-1E cells (insulinoma cell line). Development of tumor nodules was analyzed in an intrasplenic xenograft model. Results: We show that all 4 combinations have synergistic effect in vitro. These combinations lead to heterogeneous mTOR pathway inhibition and increased apoptosis. In vivo, combinations lead to decreased tumor dissemination: decreased of total hepatic tumor surface and of mean of tumor nodule size. Conclusion: These results suggest that combination of mTOR pathway inhibitors and STZ should be assessed in clinical trial. This work 
is supported by Ligue contre le cancer Savoie, Lyric: GRANT INCADGOS-4664, Groupe d'étude des Tumeurs Endocrines. Keywords: neuroendocrine, therapy, streptozotocin, mtor pathway, combination, pre-clinical study.

\section{A14 \\ Filamin-A Is Required for Somatostatin Receptor 2 (SST2) Stabilization, Signaling and Angiogenesis Regulation in Gastroenteropancreatic Neuroendocrine Tumors}

\author{
Vitali E. ${ }^{a}$, Cambiaghi V. ${ }^{\mathrm{a}}$, Loiarro F. ${ }^{\mathrm{a}}$, Zerbi A. ${ }^{\mathrm{b}}$, Colombo P., \\ Spada A. ${ }^{d}$, Mantovani G. ${ }^{d}$, Carnaghi C. ${ }^{e}$, Lania A. ${ }^{\dagger}$ \\ aLaboratory of Cellular and Molecular Endocrinology, \\ IRCCS Clinical and Research Institute Humanitas,

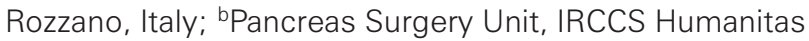 \\ Clinical Institute, Rozzano, Italy; ' Department of \\ Pathology, IRCCS Humanitas Clinical Institute, Rozzano, \\ Italy; ${ }^{d}$ Endocrine Unit, Department of Clinical Sciences \\ and Community Health, Fondazione IRCCS Ospedale \\ Maggiore Policlinico, University of Milan, Milan, Italy; \\ eMedical Oncology and Hematology Unit, Cancer Center, \\ Humanitas Clinical and Research Center, Rozzano, Italy; \\ fLaboratory of Cellular and Molecular Endocrinology, \\ BIOMETRA Department, University of Milan, \\ Neurosurgery Department, IRCCS Humanitas Clinical \\ Institute, Rozzano, Italy
}

Introduction: Somatostatin receptor type 2 (SST2) is the main pharmacological target for GEP-NETs. A subset of patients is resistant to somatostatin analogs (SSa), although the molecular mechanisms responsible for resistance are poorly understood. Several studies identified cytoskeleton protein as determinant in receptor anchoring and signaling. Aim(s): Since SST2 was demonstrated to associate with ubiquitous cytoskeleton protein Filamin A (FLNA), the aim was to investigate the role of FLNA in SST2 expression, signaling and angiogenesis in GEP-NETs and QGP1 cell line. Materials and Methods: We analyzed FLNA and SST2 expression by IHC and western blot and the role of FLNA by gene silencing. Results: We found no correlation between FLNA and SST2 expression in GEP-NETs, confirming this, FLNA knockdown did not induce changes in SST2 levels in QGP1 cells. On the contrary, the reduction in cyclin D1 levels $(-44 \pm 22 \%, \mathrm{p}<0.05 \mathrm{vs}$. basal) and ERK1/2 phosphorylation $(-59 \pm 18 \% \mathrm{p}<0.05$ vs. basal) induced by SST2 agonist (BIM23120) were abolished in FLNA lacking QGP1 cells. Similarly, BIM23120 inhibited forskolin-stimulated cAMP accumulation in control cells and this effect was abrogated in FLNA silenced cells. Moreover, FLNA knock-down reduced SST2 expression after agonist exposure $(-49 \pm 17 \%, p<0.05$ vs. basal). In addition, the inhibition of VEGF secretion by SST2 was abolished in FLNA silenced cells. Conclusion: In conclusion, these results demonstrate that FLNA is involved in SST2 stabilization, signaling and angiogenesis in GEPNETs cells. Keywords: flna, sst2, gep-net.

\section{A15 \\ Autophagy as a Possible Mechanism of Resistance in pNET Treatment}

Wiedmer T., Marinoni I., Tschan M., Perren A.

Institute of Pathology, University of Bern, Bern, Switzerland

Introduction: Pancreatic neuroendocrine tumor ( $\mathrm{pNET}$ ) patients often display primary or secondary resistance to Sunitinib, an antiangiogenic multi-receptor tyrosine kinase inhibitor which has been approved for the treatment of late stage pNETs. In late cancer stage autophagy often has a tumor promoting role. Sunitinib might additionally activate autophagy through inhibition of mTOR or induction of hypoxia. Aim(s): To assess whether autophagy acts as a mechanism of resistance in late stage pNETs and if autophagy inhibition increases therapy efficacy. Materials and Methods/Results: In pNET cell lines we show that Sunitinib induces autophagy and that the combined treatment of Sunitinib with the autophagy inhibitor chloroquine (CQ) leads to a synergistic effect in reducing cell viability. While Sunitinib and CQ alone have only minor impact on apoptosis, their combination leads to increased caspase-3/7 activity, which could explain the synergistic effect. Furthermore, in the colony formation assay Sunitinib-treated cells form as many colonies as control-treated cells, while the addition of autophagy inhibitors leads to reduction of recovery by $50 \%$. On human pNETs tissue we observe that the number of autophagosomes measured by LC3 is significantly increased in liver metastasis compared to matched primary tumors, suggesting a role of autophagy in metastasized pNETs in-vivo. Conclusion: These data indicate that in pNETs autophagy might act as a mechanism of resistance and represent an additional therapy target. Keywords: pnet, autophagy, sunitinib, resistance. 


\section{Basic Science - Genetics, Epigenetics, miRNAs}

\section{B1 \\ Cyclin-Dependent Kinase Inhibitor 2A (CDKN2A/P16) Polymorphisms and Risk of Pancreatic Neuroendocrine Tumors}

\begin{abstract}
Campa D. a, Rizzato C. ${ }^{\text {a }}$ Capurso G. ${ }^{\text {b }}$, Delle Fave G. ${ }^{\text {b, }}$ Gazouli M. ', Landi S. ', Büchler M.W. ', Malecka-Panas E. ${ }^{\dagger}$ Boggi U. ${ }^{g}$, Andriulli A. ${ }^{\text {h, }}$ Scarpa A. ${ }^{\text {, }}$ Pasquali C. ${ }^{\mathrm{j}}$, Canzian F. ${ }^{\mathrm{k}}$

${ }^{a}$ German Cancer Research Center (DKFZ) and University of Pisa, Heidelberg/Pisa, Germany/ltaly; ' bapienza' University, Rome, Italy; 'University of Athens, Athens, Greece; dUniversity of Pisa, Pisa, Italy; eUniversity Hospital Heidelberg, Heidelberg, Germany; ${ }^{f}$ Medical University of Lodz, Lodz, Poland; IUniversity Hospital of Pisa, Pisa, Italy; hIRCCS Scientific Institute and Regional General Hospital 'Casa Sollievo della Sofferenza', San Giovanni Rotondo, Italy; 'University and Hospital Trust of Verona, Verona, Italy; iUniversity of Padua, Padua, Italy; ${ }^{k}$ German Cancer

Research Center (DKFZ), Heidelberg, Germany
\end{abstract}

Introduction: Pancreatic neuroendocrine tumors (PNETs) represent only $1 \%$ of all pancreatic neoplasms by incidence, but $10 \%$ by prevalence. Family history of cancer is the only established risk factor so far. The impact of germline genetic variability on PNET risk is poorly understood. Aim(s): In this study we analyzed the genetic polymorphisms of the cyclin-dependent kinase inhibitor $2 \mathrm{~A}$ $(\mathrm{CDKN} 2 \mathrm{~A} / \mathrm{p} 16)$ gene with respect to the risk of developing the disease. Materials and Methods: We genotyped 18 single nucleotide polymorphisms (SNPs) belonging to the CDKN2A gene region in 324 PNET cases and 2126 controls recruited within the PANcreatic Disease ReseArch (PANDoRA) consortium, a well established multicentric study. Results: We found that carriers of the minor allele of SNP rs2518719 were at increased risk of developing the disease $(\mathrm{OR}=1.33 ; 95 \%$ CI $0.85-2.07 ; \mathrm{P}=0.19$ for heterozygotes; $\mathrm{OR}=$ 2.26 ; $95 \%$ CI $0.86-5.90 ; \mathrm{P}=0.09$ for homozygotes of the rare allele; Ptrend $=0.006$ ). This association is potentially important since CDKN2A/p16 plays a key role in the regulation of cell cycle and since both rare mutations and common SNPs in the gene region have been associated with the risk of developing multiple cancer types. Conclusion: In conclusion we performed the largest study to date on the genetic susceptibility of PNETs and we highlight the association between a common genetic variant of a pleiotropic gene and an increased risk of developing the disease. Keywords: pancreatic neuroendocrine tumor, p16, cdkn2a, genetic polymorphisms, snp, association, susceptibility.
B2

\section{Molecular Profiling of Small Intestinal} Neuroendocrine Tumours (SINETs)

Karpathakis A. ${ }^{a}$, Feber A. ${ }^{a}$, Morris T. ${ }^{a}$, Dibra H. ${ }^{\text {a, }}$ Pipinikas C. ${ }^{\text {, }}$ Francis J. ${ }^{\mathrm{b}}$, Mandair D. ${ }^{\mathrm{c}}$, Toumpanakis C. ${ }^{\mathrm{c}}$, Meyer T. ${ }^{a}$ Luong T. ${ }^{\mathrm{c}}$, Caplin M. ${ }^{\mathrm{c}}$, Meyerson M. ${ }^{\mathrm{b}}$, Beck S. ${ }^{\mathrm{a}}$, Thirlwell $C$. $^{\text {a }}$

aUCL Cancer Institute, London, UK; bThe Broad Institute, Boston, Mass., USA; ' Royal Free Hospital NHS Trust, London, UK

Introduction: Aberrant DNA methylation plays an role in the pathogenesis of many cancers, however little is known about its role in SINET development. Aim(s): We report the first unbiased genomewide DNA methylation analysis of a large cohort of SINET, aiming to identify epimutations specific to SINET which may contribute to tumorigenesis. Materials and Methods: HumanMethylation450 Array analysis was performed on DNA from SINET tumours $(\mathrm{n}=$ $49)$ and normal SI $(n=21)$. Publicly available methylation data on $>600$ intestinal adenocarcinoma was also assessed for comparison. Gene expression was determined using the DASL array on RNA from SI NET tumours $(n=32)$ and normal SI $(n=6)$. Analysis was performed using ChAMP and limma $\mathrm{R}$ packages. A Bonferroni adjusted significance threshold of $p<0.05$ was used throughout. Results: Comparison of SINET with normal SI identified a total of 130,083 Methylation Variable Positions, whilst 626 genes were found to be have $>3$ fold differential expression. Integrated analysis identified a group of 11 candidate genes where altered methylation and expression was significant (downregulated: CDX1, FBP1, C20orf54, GATA5; upregulated: PTPRN, PCSK1, PRLHR, CELSR3, GIPR, LMX1B, SCGN). Hypermethylation of GIPR affected $92 \%$ of SINETs and was sensitive for the detection of SINET with an AUC of 0.99. Conclusion: This is the first comprehensive analysis of the epigenetic profile of SINET and identifies hypermethylation of GIPR as a potential novel biomarker. Keywords: epigenetics, methylation, gipr, biomarker.

B3

Genomic Profiling of Metastatic

Gastroenteropancreatic Neuroendocrine Tumor (GEP-NET) Patients in Precision-Medicine Era

Kim S., Yoo K., Jung K., Lee S., Lee J., Park S., Park J., Lim H., Kang W., Park Y.

Samsung Medical Center, Seoul, South Korea

Introduction: We have conducted molecular profiling through high-throughput molecular test as part of clinical practice for patients with advanced gastrointestinal (GI) cancer, or rare cancer including gastroenteropancreatic neuroendocrine tumors (GEP-NETs). Aim(s): Herein, we reported the molecular characterization for 15 metastatic GEP-NET patients. Materials and Methods: We conducted the Ion AmpliSeq Cancer Hotspot Panel v2 (detecting 2,855 oncogenic muta- 
tions in 50 commonly mutated genes) and nCounter Copy Number Variation Assay (designed a 21-genes based on available targeted agents) as high throughput genomic platform in 14 patients with metastatic GEP-NETs. Results: Among the 14 GEP-NET patients analyzed in this study, 5 patients had grade III neuroendocrine carcinoma (NEC) and 9 grade I/II NET. Primary sites included pancreas $(\mathrm{n}=3)$, small intestine and ascending colon $(\mathrm{n}=3)$, distal colon and rectum $(n=5)$, and unknown primary origin $(n=3)$. Most common metastatic site was liver. Of 14 patients available for mutational profiling, the common aberrations were as follows: SMARCB1 mutation $(\mathrm{n}=2)$; TP53 mutation $(\mathrm{n}=2)$; STK11 mutation $(\mathrm{n}=1)$; RET mutation $(\mathrm{n}=1)$; and BRAF mutation $(\mathrm{n}=1)$. Gene amplification by nCounter was detected in only 1 patient, showing CCNE1 amplification. There was no any mutation and amplification of gene in 7 patients. Conclusion: This high throughput genomic test may be useful to identify new drug targets in metastatic GEP-NET patients. Keywords: molecular profiling, gep-net.

\section{B4}

\section{A Novel Human Cell Line from Familial Medullary Thyroid Carcinoma}

Pfragner R. ${ }^{a}$, Schwach G. ${ }^{a}$, Hoeger H. ${ }^{\text {, }}$ Ghaffari Tabrizi-Wizsy N. ", Tam Amersdorfer C. ${ }^{\text {, }}$ Leitinger G. ${ }^{\mathrm{c}}$, Bock E. ', Zeitlhofer P. , Haas O.A. ${ }^{\mathrm{d}}$, Beichler C. ${ }^{\mathrm{e}}$, Speicher M.R. ${ }^{\mathrm{e}}$, Rinner B. ${ }^{\mathrm{f}}$, Niederle B. ${ }^{\mathrm{g}}$, Strob/ H.

alnstitute of Pathophysiology and Immunology, Medical University of Graz, Austria; 'b Core Unit of Biomedical Research, Division of Laboratory Animal Science and Genetics, Medical University of Vienna, Austria; Institute of Cell Biology, Histology and Embryology and Core Facility Ultrastructure Analysis, Medical University of Graz, Austria; ${ }^{d}$ medgen.at $\mathrm{GmbH}$, Vienna, Austria; eInstitute of Human Genetics, Medical University of Graz, Austria; ${ }^{f}$ Center for Medical Research, Medical University of Graz, Austria; ' Endocrine Surgery, Division Geneal Surgery, Medical University of Vienna, Austria

Introduction: Cell lines from hereditary medullary thyroid carcinomas (MTC) are very rare, therefore, there is a substantial need to establish new cell lines. Here, we present a novel familial MTC (FMTC) cell line, designated SCHWE. Aim(s): The aim of this study was to establish and characterize an in vitro and in vivo model of FMTC. Materials and Methods: Tissue was obtained from a primary tumor of a 50 year old male. DNA was isolated from peripheral blood and from tumor tissue. Sequence analysis revealed a RET-mutation in Exon 13, Codon 781 (CAG>CGG) and Codon 769 (CTT $>$ CTG). SCHWE cells were long term cultured and analyzed by microscopy, electron microscopy, immunofluorescence, FACS analysis and Array-CGH. To proof the tumorigenicity immumnodeficient NODscid gamma mice were injected with SCHWE cells. Results: In culture, the SCHWE cells formed monolayers of epithelial cells, population doubling time was approximately 10 days. Cells exhibited the characteristic ultrastructure of MTC, were calcitonin, chromogranin A and gastrin-releasing peptide positive, showed a tetraploid level and chromosomal imbalances. SCHWE cells were tumorigenic, rapid tumor growth was observed in xenografts. Conclusion: The SCHWE cell line represents a specific model for studies of hereditary MTCs. This new in vitro model and the appropriate mouse model may contribute to a better understanding of cell biology, genetics, tumor progression and can also serve as as preclinical model for the development of anticancer agents. Keywords: novel mtc cell line, familial mtc, xenografts.

\section{B5}

\section{Comparative Proteomic Analysis of Human Insulinoma and Its Clinical Implications in Pancreatic Neuroendocrine Tumors}

\author{
Qiao X.W. ${ }^{\text {, }}$ Song Y.L. ${ }^{a}$, Bai C.M. ${ }^{\text {, }}$, Zhu L.M. ${ }^{a}$, Lu C.M. ${ }^{a}$, \\ Xiao Y. ${ }^{\mathrm{a}}$, Chen J. ${ }^{\mathrm{b}}$, Wang Y.H. ${ }^{\mathrm{b}}$, Tang L. ${ }^{\mathrm{c}}$, Yu R. ${ }^{\mathrm{d}}$, Chen Y.J. ${ }^{\mathrm{a}}$ \\ aDepartment of Gastroenterology, Peking Union Medical \\ College Hospital, Peking Union Medical College, Chinese \\ Academy of Medical Science, Beijing, China; 'bepartment \\ of Gastroenterology, The First Affiliated Hospital of Sun \\ Yat-Sen University, Guangzhou, China; ' Department of \\ Pathology, Memorial Sloan-Kettering Cancer Center, \\ New York City, N.Y., USA; dDepartment of Endocrinology, \\ Cedars-Sinai Medical Center, UCLA, Los Angeles, Calif., \\ USA
}

Introduction: Insulinoma is the most common functional PNET. Its molecular tumorigenesis is largely unknown and proteomic study on insulinoma has not been reported. Aim(s): To identify the differential expression of proteins in insulinoma and normal pancreas by comparative proteomic analysis, to evaluat the clinicopathologic implications of certain proteins expressions in insulinomas and PNET. Materials and Methods: Proteins isolated from 5 fresh frozen insulinoma tissues and 4 normal pancreatic tissues were used for Nanospray LC/MS/MS analysis. 294 PNETs from 286 pts were enrolled and 230 pts $(80 \%)$ were followed up. The expressions of protein UCH-L1, p53BP1, MAP2, PDX-1, and $\alpha$-internexin (INX) were validated in insulinoma and other PNET by IHC and western blot. Expressions of both UCH-L1 and INX were correlated with clinicopathologic features and prognosis. Results: More than 1000 proteins were differentially expressed, 1942 proteins were specifically expressed in insulinomas and 249 proteins were found in normal pancreatic tissues. The expressions of 5 proteins UCH-L1, MAP2, PDX-1, p53BP1 and INX were confirmed in validation group. The expression of both UCH-L1 and INX protein in PNETs was significantly associated with lower stage $\mathrm{P}=1.6 \times 10-4$, better overall survival and disease free survival, $\mathrm{P}=0.006$ and $\mathrm{P}=1.4 \times 10-4$, respectively. Conclusion: A number of proteins differentially expressed in PNET might contribute to their tumorigenesis. The protein UCH-L1 and INX could be prognostic biomarker of PNET. Keywords: proteomics, pnet, uch-11, $\alpha$-internexin, prognosis. 


\section{B6}

\section{Functional Role of miR-196a in Neuroendocrine Tumor Cells}

\author{
Shi H. ${ }^{a}$, Li S.C. ${ }^{a}$, Khan M. ${ }^{b}$, Caplin M. ${ }^{b}$, Meyer T. ${ }^{c}$, Öberg K. ${ }^{a}$ \\ Giandomenico V. ${ }^{\text {a }}$ \\ a Uppsala University, Uppsala, Sweden; broyal Free \\ Hospital, London, UK; ' University College London Cancer \\ Institute, London, UK
}

Introduction: An exclusive miRNA profiling of small intestinal neuroendocrine tumors (SI-NETs) was published in 2013; and nine miRNAs were selected for further investigations. We chose miR-196a to elucidate its target genes and biological functions, by using NET cells as in vitro models. Aim(s): To identify miR-196a target genes and explore their biological functions. Materials and Methods: MiR-196a targets prediction analysis suggested four target genes for further investigation. We used anti-miR-196a to silence CNDT2.5 and NCI-H727 cells. Target genes expression and encoded proteins were analyzed by quantitative real-time PCR and western blot analyses. Furthermore, migration assay was performed. Results: Four target genes, HOXA9, HOXB7, LRP4 and RSPO2, are significantly increased in miR-196a silenced CNDT2.5 and NCI-H727 cells at transcriptional and translational levels. In addition, six downstream genes, BMP4, ETS-1, CTNNB1, FZD5, LRP5 and LRP6, are also upregulated at transcriptional level in miR-196a silenced NET cells. Conclusion: We have identified 4 target genes and 6 downstream genes regulated by miR-196a in NET cells. MiRNA target genes functional study is requested to elucidate SI-NET progression mechanisms and provide potential therapeutic targets. Keywords: neuroendocrine tumors, mirna target genes, mirna silencing.

\section{B7 \\ Exclusive Hotspot Mutation of YY1 Gene in Insulinomas and Extensive Mutation of DAXX in PNETs}

\author{
Song Y.L. ${ }^{a}$, Qiao X.W. ${ }^{a}$, Bai C.M. ${ }^{a}$, Lu C.M. ${ }^{\text {a }}$, Chen J. ${ }^{\text {, }}$ \\ Yu R. ${ }^{\text {, }}$ Tang L. ${ }^{d}$, Chen Y.J. ${ }^{\text {a }}$ \\ aDepartment of Gastroenterology, Peking Union Medical \\ College Hospital, Chinese Academy of Medical Sciences, \\ Beijing, China; 'bepartment of Gastroenterology, The First \\ Affiliated Hospital, Sun Yat-sen University, Guangzhou, \\ China; 'Department of Endocrinology, Cedars Sinai \\ Medical Center, Los Angeles, Calif., USA; 'Department of \\ Pathology, MSKCC, New York City, N.Y., USA
}

Introduction: Somatic mutation of YY1 was shown in sporadic insulinoma, the main type of functional PNET. DAXX mutation occurred mainly in NF-PNET. However, YY1 and DAXX mutations in non-insulinomas and insulinomas, respectively, have not been well elucidated. Aim(s): To assess somatic mutations of YY1 and DAXX in PNET. Materials and Methods: DNA from 132 tumor tissues (37 NF, 77 insulinomas, 11 gastrinomas, 5 glucagonomas, 2 VIPomas) were extracted. YY1 gene was sequenced in all 132 PNET,
DAXX gene was sequenced in 81 (45 insulinomas and 36 non-insulinomas). IHC staining of YY1 was performed in all PNET. Data were correlated with clinicopathologic features. Results: Hotspot mutation of YY1 (c.C1115G/p.T372R) was found in 14 of 77 insulinomas but only in 1 of 55 non-insulinomas ( $18 \%$ vs. $1.8 \%, p=0.002)$. In 81 PNETs, DAXX mutations were found in 17 of 45 insulinomas and in 22 of 36 non-insulinomas ( $38 \%$ vs. $61 \%, p=0.015)$. YY1 mutation were shown in 7 of 45 insulinomas and none in 36 non-insulinomas $(p=0.046)$. Two tumors had both genes mutations. YY1 expression was shown in all PNETs with/wo mutated YY1. DAXX mutation was more common in female $(\mathrm{P}=0.044)$ while more $\mathrm{YY} 1$ mutation shown in older pts $(\mathrm{P}=0.02)$. Neither YY1 nor DAXX mutation was associated with tumor size, grade and stage. Conclusion: Half of insulinoma had either YY1 or DAXX mutation while YY1 mutation was rare in non-insulinoma. Our findings suggest DAXX and YY1, a target of mTORC1, could be important in insullinoma tumorigenesis, and potential therapeutic target. Keywords: yy1, daxx, mutation, pnet.

B8

\section{Epigenetic Manipulation of the Somatostatin Receptor Type 2 in Neuroendocrine Tumor Cells}

Veenstra M.J. ${ }^{a}$, Van Koetsveld P.M. ${ }^{\text {a }}$ Dogan Fa,

Farrell W.E. b, Vitale G. ${ }^{c}$, Lamberts S.W.J. ${ }^{\text {, }}$ De Herder W. ${ }^{\text {, }}$ Hofland L.J. ${ }^{\text {a }}$

aDepartment of Internal Medicine, Division of Endocrinology, Erasmus Medical Center, Rotterdam, The Netherlands; 'bepartment Human Disease and Genomics Group, Institute of Science and Technology in Medicine, School of Medicine, Keele University, Stoke on Trent, Staffordshire, UK; ' Laboratory of Endocrine and Metabolic Research, Istituto Auxologico Italiano IRCCS, Department of Clinical Sciences and Community Health (DISCCO), Milan, Italy

Introduction: The somatostatin receptor type 2 (sst2) is a target for treatment in patients with neuroendocrine tumors (NET). However, variability in tumoral sst 2 expression might lead to variability in response. Epidrugs could increase sst2 levels and improve response to somatostatin analogues (SSA). Aim(s): Determine the effect of the DNA demethylating drug 5-aza-2'-deoxycytidine (5-aza-dC) and histone deacetylase inhibitor Valproic Acid (VPA) on sst2 mRNA, internalization and cAMP signaling in NET cell lines BON and QGP. Materials and Methods: After 7 days treatment with 5-aza-dC, VPA or both, sst2 mRNA values were determined by Q-RT-PCR, as well as the effect on uptake of [125I-Tyr3]octreotide and in BON on the inhibition of forskolin-stimulated cAMP expression by octreotide. Results: In BON both compounds stimulated sst2 mRNA expression, while in QGP 5-aza-dC was stimulatory and VPA inhibitory. Both drugs significantly increased uptake of [125ITyr3]octreotide in BON and QGP. In BON both drugs increased the potency of octreotide to inhibit cAMP levels (lowered IC50). Conclusion: In BON and QGP we found variable response to epidrug treatment, stressing a difference between BON and QGP that 
might resemble NET heterogeneity. We showed that treatment with epidrugs enhanced cAMP response to SSA, as well as internalization of radiolabeled octreotide. Such effects can be clinically relevant in terms of hormonal control by SSA, as well as for PRRT. Keywords: net, sst2, epidrug, signaling.

B9

\section{Androgens Regulate SMAD Ubiquitination Regulatory Factor-1 Expression and Prostate Cancer Cell Invasion}

\author{
Xiaokun G., Guixia W. \\ Department of Endocrinology and Metabolism, \\ Changchun, China
}

Introduction: Prostate cancer is the most commonly diagnosed male cancer and is a hormone-driven disease. Androgens have been recognized as a major promoter of $\mathrm{PCa}$ development and progression. However, the mechanism of androgen action in $\mathrm{PCa}$, especially in $\mathrm{PCa}$ cell invasion remains largely unclear. SMAD ubiquitination regulatory factor-1 (SMURF1) is a C2-WW-HECT-domain E3 ubiquitin ligase that plays important roles in cancer cell metastasis. Whether there is a relationship between androgens and SMURF1 express is not known. Aim(s): Identification of novel therapeutic targets for treatment of advanced PCa. Materials and Methods: The effect of androgens on the expression of SMURF1 in PCa cell lines was examined by Western blot and RT-PCR. The binding of AR to the SMURF1 gene enhancer was determined by chromatin immunoprecipitation (ChIP) assay, CHIP-Seq and RNA-Seq analysis. Cell migration and invasion was measured by wound healing and Matrigel invasion assays, respectively. Results: The expression of SMURF1 is upregulated by androgens in PCa cell lines and this effect is mediated through androgen receptor (AR). Androgens regulate SMURF1 expression at transcriptional level and AR transcriptionally activates SMURF1 by binding to its enhancer that contains a canonical half androgen responsive element (ARE). SMURF1 is important for androgen-induced invasion of PCa cells. Conclusion: SMURF1 is a bona fide target gene of the AR. Our findings also suggest a potential role of SMURF1 in PCa metastasis. Keywords: androgen, ar, smurf1, prostate cancer, invasion.

\section{Basic Science - In vitro Models, Tumor Growth, CTCs}

\author{
C1 \\ Investigation of the Effects of Sunitinib on \\ Pheochromocytoma and Paraganglioma \\ Primary Cultures \\ Bellio M., Gagliano T., Feo C., Balboni F., Gentilin E., \\ Degli Uberti E., Zatelli M.C. \\ University of Ferrara, Ferrara, Italy
}

Introduction: The main treatment for Pheochromocytoma and Paraganglioma is surgery. However these tumors are highly vascularized, suggesting the possible role for anti-angiogenic agents in the medical therapy. Sunitinib is a multi-targeted receptor tyrosine kinase inhibitor (TKI), mainly described to inhibit VEGFR. Aim(s): To verify the effects of Sunitinib on human Pheochromocytoma and Paraganglioma primary cultures. Materials and Methods: 5 primary cultures (4 Pheochromocytoma and 1 Paraganglioma) were obtained from patients, followed in our centre, whose data about age, genetic alteration, histological features, and clinical events had been collected. Cell viability and apoptosis were measured by ATPlite and Caspase 3/7 assay. Results: Patients ( 2 females and 3 males) were $52.6 \pm 4.4$ years old and were hypertensive; 2 displayed germ-line RET mutations (and had a medullary thyroid carcinoma). All lesions measured $>1 \mathrm{~cm}$ diameter. Sunitinib was capable of inhibiting cell viability ( $-40 \%$ vs. ct $\pm 20 \%$ ), and activating caspase $3 / 7$ ( $+100 \%$ vs. ct $\pm 20 \%$ ). Conclusion: In our series Sunitinib was capable of reducing cell viability and activating the apoptotic process, independently of genetic alterations. Even if more data are needed to support the efficacy of this treatment, our preliminary data suggest a possible role for Sunitinib in Pheochromocytoma and Paraganglioma medical treatment. Keywords: sunitinib, pheochromocytoma, paraganglioma. 
C2

\section{Zebrafish as a New in vivo Model to Study Angiogenesis in Neuroendocrine Tumors (NET)}

\author{
Gaudenzi G. ${ }^{\text {a }}$ Dicitore A. ${ }^{\text {b }}$, Cotelli F. ${ }^{\mathrm{c}}$, Ferone D. ${ }^{\mathrm{d}}$, \\ Persani L. ${ }^{a}$, Vitale G. $^{\text {a }}$ \\ aDepartment of Clinical Sciences and Community \\ Health, University of Milan, Milan, Italy; 'baboratory \\ of Experimental Endocrinology, Istituto Auxologico \\ Italiano IRCCS, Milan, Italy; ' Department of Biosciences, \\ University of Milan, Milan, Italy; ${ }^{d}$ Department of Internal \\ Medicine and Medical Specialties, University of Genoa, \\ Genoa, Italy
}

Introduction: Zebrafish is a powerful model to study human diseases, including cancer. Aim(s): A novel in vivo angiogenesis assay based on the injection of human NET cell lines (TT, MZ-CRC1, DMS79 and PC3) and primary NET cell cultures has been developed in $\operatorname{Tg}($ fli1:EGFP)y1 zebrafish embryos, in which the fluorescent protein EGFP is expressed in the vascular tree. Materials and Methods: NET cells were stained with red fluorescent dye and injected in the proximity of the developing sub intestinal vessel (SIV) plexus in $\mathrm{Tg}$ (fli1:EGFP)y1 embryos. The vasoproliferative response has been detected by both fluorescence microscopy and alkaline phosphatase staining at 24-48 hours post injection. Results: While uninjected embryos (control group) displayed a normal SIV plexus, the injection of NET cells stimulated the sprout of new vessels from the SIV toward the tumor graft. Due to the easy and rapid visualization of the tumor angiogenesis, we are currently using this model to test the effects of antiangiogenic compounds on NET induced vascularization. In addition, the use of fluorescently labelled NET cells provides to investigate their metastatic behavior after transplantation. Indeed the transparency of the zebrafish embryos allows to follow the very early steps of invasion, circulation of tumour cells in blood vessels and metastasis formation in real-time. Conclusion: This model may represent in future an innovative treatment assay able to provide information for the most appropriate and personalized treatment in patients with NET. Keywords: zebrafish, net.

\section{C3 \\ Mitotane, Adrenolitic Drug, Inhibits Cell Survival and Function of Several Pituitary Cytotypes}

Gentilin E., Gagliano T., Benfini K., Di Pasquale C., Falletta S., Ambrosio M.R., Degli Uberti E., Zatelli M.C.

Department of Medical Sciences, Section of

Endocrinology, Ferrara, Italy

Introduction: Mitotane (DDD) is an adrenolytic agent that is used for the treatment of adrenocortical carcinoma. We previously demonstrated that DDD affects thyrotrope cell viability and function. These data represent a possible explanation of the biochemical picture consistent with central hypothyroidism in patients undergo- ing DDD therapy. DDD also inhibits corticotrope cell viability by inducing caspase-mediated apoptosis and reduces POMC expression as well as basal and CRH-induced ACTH secretion. Cells originating from tissues different from pituitary are not sensitive to the inhibitory effects of DDD. Our data suggest that DDD inhibits cell survival and function of many pituitary cytotypes, acting with a generalized, but specific, toxic effect. The majority of male patients undergoing adjuvant DDD therapy show a clinical picture of hypogonadism, characterized by low free testosterone and unmodified LH concentration. $\operatorname{Aim}(\mathbf{s})$ : We investigated whether mitotane may affect gonadotroph cells. Materials and Methods: We evaluated cell viability, apoptosis, cell cycle and hormonal secretion. Results: We found that mitotane reduces cell viability, induces caspase $3 / 7$ activity, modifies cell cycle phase distribution and LH/FSH secretion of gonadotroph cells. Conclusion: The present data strengthen previous evidence showing a mitotane hypopituitary effect and represent a possible explanation for the lack of LH increase following free testosterone decrease in patients undergoing adjuvant mitotane therapy. Keywords: mitotane, adrenocortical cancer, pituitary.

\section{C4 \\ Cancer Stem Cells in Small Intestine Neuroendocrine Cell Line P-STS: Isolation and Molecular Characterisation}

\author{
Kleinegger F., Meier-Allard N., Fuchs R., Hölzl M., \\ Krump C., Passegger C., Pfragner R., \\ Ghaffari Tabrizi-Wizsy N. \\ Institute of Pathophysiology and Immunology, Medical \\ University of Graz, Graz, Austria
}

Introduction: Cancer stem cells (CSCs) represent a small subpopulation of tumour cells responsible for invasive tumour growth. Even though the presence of CSCs in neuroendocrine tumours is documented, their role in neuroendocrine tumourigenesis and metastasis is not defined as yet. $\mathbf{A i m}(\mathbf{s})$ : The aim of this study was first to characterise the SI-NET cell line P-STS regarding CSC characteristics and second, to test whether CSC capacity impacts tumourigenesis and invasive behaviour of the cells. Materials and Methods: Aldehyde dehydrogenase (ALDH) activity was detected using the Aldefluor kit. Further potential CSC markers (CD133, CD24, CD44, CD117, CD15, Nanog, Sox2, Oct4, MSI1, BMI1, ID-1 and Nestin) were analysed by flow cytometry and/or RT-PCR. A spheroid-forming assay was performed in order to simulate tumour like conditions in vitro. The invasiveness of CSCs was assessed in vivo using the chick chorioallantoic membrane (CAM) assay. Results: A small population (1.8\%) with high ALDH activity was detected in the P-STS cell line. P-STS cells expressed CSC specific genes, which mostly were up-regulated in spheroids. The invasion rate of the cells was enhanced due to $3 \mathrm{D}$ cultivation. Conclusion: Our data provide first evidence that the P-STS cell line exhibit various characteristics of CSC cells. Thus, the P-STS cell line represents a valuable model for studying cells with $\mathrm{CSC}$ characteristics in SI-NET research. The role of the ALDH+ fraction needs to be defined in future experiments. Keywords: cancer stem cells, tumor growth, tumorigenesis \& metastasis. 
C5

\section{The Impact of Pharmacological-Mediated Depletion of Macrophages in Pancreatic Neuroendocrine Neoplasms}

Krug S. ${ }^{a}$, Abbassi R. ${ }^{a}$, Rinke A. ${ }^{a}$, Griesmann H. ${ }^{\mathrm{b}}$, Gress T. Michl P.

aDepartment of Gastroenterology and Endocrinology, University of Marburg, Germany; 'bepartment of Gastroenterology and Endocrinology, University Hospital of Marburg, Germany

Introduction: Pancreatic neuroendocrine tumors (PNETs) are a heterogeneous group of neuroendocrine neoplasms with distinct biological behaviour and response to treatment. Previous reports indicate that the infiltration of tumor-associated macrophages (TAMs) in PNETs strongly correlates with proliferation and metastases. Aim(s): Evaluation of the impact of the liposomal clodronate (clodro) as pharmacological tool for macrophage depletion in-vitro and in-vivo. Materials and Methods: The effect of clodro on cell-viability was analysed in J774 and RAW myeloid cells and isolated murine bone macrophages as well as CD11b+ cells of RIP1-Tag2 pancreata which were evaluated by FACS. RIP1-Tag2 mice were treated with either clodro or liposomes alone to evaluate tumor progression, proliferation, angiogenesis and macrophage infiltration by FACS and immunohistochemistry. Results: Clodro inhibited the proliferation of J774 and RAW cells and murine bone macrophages. In contrast, cellviability of Bon-1 and Ins1 cells was not affected by this treatment. FACS analyses of RIP1-Tag2 mice treated with clodro confirmed the solitary depletion of macrophages. In RIP1-Tag2 mice clodro reduced the evolution of invasive beta-cell tumors. Furthermore, proliferation of tumor cells and angiogenesis within the tumors were reduced. The mechanism by which the regulation of macrophages suppresses tumor hallmarks is currently under investigation. Conclusion: Clodro selectively depletes macrophages and disrupts tumor progression in the RIP1-Tag2 tumor model. Keywords: clodro, tam, rip1-tag2.

\section{C6 \\ Establishment of a New Human Pancreatic Neuroendocrine Tumour Cell Line}

\author{
Schrader J. ${ }^{a}$, Benten D. a, Rhotert C. ${ }^{\text {, }}$ Fahl M. ${ }^{\text {a }}$ Perez D. ${ }^{\text {, }}$ \\ Bockhorn M. ${ }^{\mathrm{b}}$, Izbicki J. ${ }^{\mathrm{b}}$, Lohse A. ${ }^{\mathrm{a}}$ \\ aDepartment of Internal Medicine, University Medical \\ Center Hamburg-Eppendorf, Hamburg, Germany; \\ bDepartment of General, Visceral and Thoracic Surgery, \\ University Medical Center Hamburg-Eppendorf, Hamburg, \\ Germany
}

Introduction: The development of new therapeutic strategies for patients with pancreatic neuroendocrine tumours is impaired by the paucity of suitable pre-clinical models. Although the two available tumour cell lines BON and QGP have a neuroendocrine phenotype, the proliferation rate is too high for a well differentiated neuroendocrine tumour. $\operatorname{Aim}(\mathbf{s})$ : Therefore the aim was to establish new neu- roendocrine cell lines from patient-derived tumour tissue. Materials and Methods: We here report the successful establishment and inital characterisation of a new neuroendocrine cell line (called NT-3) derived from a lymphnode metastasis from a patient with a pancreatic neuroendocrine tumour. Results: The NT-3 cells grow adherent in grape-like structures. They have been cultured for more than 10 passages with a stable neuroendocrine phenotype. The cells have been successfully cryo-preserved and thawed. The doubling time of NT-3 is more than 14 days, which can be shortened to 8 days by the addition of growth factors. This is in stark contrast to a doubling time of less than 2 days for BON and QGP cells. Chromogranin A expression is 20- and 200-fold higher in NT-3 compared to BON and QGP, respectively. In addition the new cell line has a more than 10 -fold higher expression of VEGF-A and VEGF-B than BON and QGP cells. Conclusion: In summary, we have established a new slow growing pancreatic neuroendocrine tumour cell line with a well differentiated phenotype and high expression of pro-angiogenic factors. Keywords: neuroendocrine, cell line, pancreas.

\section{Epidemiology/Natural History/ Prognosis - Registries, Nationwide and Regional Surveys}

D1

\section{The Belgian DNET Registry: A Prospective, National, Web-Based Registry of Digestive Neuro-Endocrine Tumours (NET). Status after 2 Years of Inclusion}

\begin{abstract}
Borbath I. a, Dresse D. ${ }^{\mathrm{b}}$, Verslype C. ${ }^{\mathrm{c}}$, Demolin G. ${ }^{\mathrm{d}}$, Rezai Kalantari H. e, Geboes K. ${ }^{\dagger}$, Moons V. ${ }^{\mathrm{g}}$, Polus M. h, Van Laethem J.L.', Peeters M.', Bols A. k, Demey W.', Lambrechts G. ${ }^{m}$, Van Cutsem E. ${ }^{\mathrm{c}}$

${ }^{a}$ Cliniques Universitaires Saint-Luc, Bruxelles, Belgium; ${ }^{b}$ CHR La Citadelle, Liège, Belgium; ' UZ Leuven, Leuven, Belgium; ${ }^{d} \mathrm{CHC}$ St-Joseph, Liège, Belgium; ${ }^{e} \mathrm{CH}$ Peltzer-La Tourelle, Verviers, Belgium; 'UZ Gent, Gent, Belgium; Imelda Hospital, Bonheiden, Belgium; ${ }^{h} \mathrm{CHU}$ Sart Tilman, Liège, Belgium; 'Hopital Erasme, Bruxelles, Belgium; iUZA, Antwerpen, Belgium; ' $A Z$ St. Jan Brugge, Brugge, Belgium; 'KLINA, Brasschaat, Belgium; mAZ Damiaan, Oostende, Belgium
\end{abstract}

Introduction: NET are rare neoplasms. The Belgian Group of Digestive Oncology created a web-based registry to have an overview on incidence, diagnosis and therapies performed in Belgian pts. The DNET registry was launched in 01/2012. Aim(s): We report data after 2 years of inclusion. Materials and Methods: Patients diagnosed with a NET after 01/01/2001 were included. As of 30/11/2014, 510 pts and more than 2000 visits were included in the registry, from 28 active sites. Results: Pts consisted of 267 men and 243 female. Mean age was $60 \pm 13$ y. ECOG PS was 0 in 245 pts (47.8\%), 1 in 133 $(26 \%), 2$ in $23(4.5 \%), 3$ in $23(4.5 \%)$ and not known in 85 . Primary 
tumours were mainly pNET $(\mathrm{n}=161,31.4 \%)$, midgut $(\mathrm{n}=165$, $32.3 \%$ ). CgA was tested in 294 patients and NSE in 177. Functional syndrome was present in $79 / 510$ pts $(15 \%)$, being carcinoid syndrome in $54(10 \%)$. Pathology was available for $89 / 165$ midgut pts $(54 \%)$ and $117 / 161$ pNET (73\%). Midgut pts were mainly WHO 2010 NETG1 $(51 / 89,57 \%)$ or NET G2 $(32 / 89,36 \%)$. pNET pts were NEC G3 (29/117, 24\%), NET G2 (60/117, 51\%) and NET-G1 (29/117, 25\%). Surgery ( 270 answers as of $30 / 11 / 2014)$ was performed in a curative intent in $217 \mathrm{pts}$, for palliative reasons in $31 \mathrm{pts}$. Initial medical therapy was chemotherapy for $39 \mathrm{pts}$, SMS analogues for $120 \mathrm{pts}$ and targeted therapy for $12 \mathrm{pts}$. During follow-up, $26 \mathrm{pts}$ died, mainly from PD, after a median $12 \mathrm{~m}(2-105)$. Conclusion: The DNET registry is recruiting well, with a $20 \mathrm{pts} / \mathrm{m}$ inclusion rate. More data will be made available at the time of congress. Keywords: registry, belgium.

\section{D2 \\ United Kingdom NET Patient Survey Quality of Life Results}

Bouvier C. ${ }^{a}$ Sissons M. ${ }^{a}$, Srirajaskanthan R. ${ }^{b}$

aNET Patient Foundation, Oxford, UK; ' ${ }^{\text {Kings College }}$ London, London, UK

Introduction: QoL is of paramount importance in patients diagnosed with cancer, this is especially true in patients with NETs since the median survival from diagnosis is 7 years. Aim(s): This survey was conducted to assess the impact of NETs on the QoL of patients. Materials and Methods: INCA in conjunction with Novartis has performed the first global survey of patients with NETs. Hall \& Partners fielded and analysed the results. The UK data reported in this abstract forms part of a much larger global survey which recruited $>1900$ patients. The UK data reported is based on 156 patients; average age $=57,83 \%$ from England. Results: Almost all patients felt their QoL was negatively affected by the diagnosis of NET. Key negative effects on QoL: are energy levels (70\%), limiting travel due to symptoms and emotional health (56\% and $51 \%$ respectively). $87 \%$ of patients had at least 1 or more symptom. Majority of these symptoms are GI symptoms, followed by anxiety and depression. Nearly all patients had to make lifestyle changes; dietary adjustments $(62 \%)$, decreasing physical activity (51\%). The median age of diagnosis was 57 year in the UK and $>2 / 3$ were of working age at time of diagnosis. Of patients not currently working, $94 \%$ had to stop working due to illness. Patients would like more information regarding long term impact of NET on their QoL and also access to support and expertise. Conclusion: It is important to take a holistic view to patient care and address the QoL issues. Keywords: quality of life, net.

\section{D3 \\ Incidence of Neuroendocrine Neoplasms in Norway: A Report of 16258 Cases from 1993 through 2010}

Boyar Cetinkaya R. ${ }^{\text {a }}$ Aagnes B. ${ }^{\mathrm{b}}$, Thiis-Evensen E. ${ }^{\mathrm{a}}$, Tretli S. ${ }^{b}$, Bergestuen D.S. ${ }^{a}$, Hansen S. ${ }^{b}$

aDepartment of Gastroenterology, Rikshospitalet, Oslo University Hospital, Oslo, Norway; ${ }^{b}$ Cancer Registry of Norway, Institute of Population-Based Cancer Research, Oslo, Norway

Introduction: Studies show an increasing trend in the incidence of neuroendocrine neoplasms (NENs). A significant number of NENs occur in less common primary sites, but they are often excluded from the population based studies. Aim(s): We studied the incidence trends of NENs in Norway according to different primary sites, including rare cases. Materials and Methods: Our analyses were based on cancer cases diagnosed from 1993 through 2010 and reported to the national population-based Cancer Registry of Norway (CRN). A total of 70 morphological codes were identified as neuroendocrine and stratified into 3 different groups of aggressiveness; low, intermediate and high. Results: We identified 16258 NENs of which $49.4 \%$ were in women. Median age at diagnosis was 65 years. The most common primary sites were the lung $(47.6 \%)$ and the gastroenteropancreatic system $(17.8 \%)$. Stage was local in $45 \%$ of the cases, regional in $16 \%$, distant in $39 \%$ and the distribution was stable throughout the study period. Age standardized (European) incidence rate (per 100,000 person years) increased from 13.5 to 21.6 from 1993 to 2010 with an estimated annual increase of $5.1 \%$ in women and $2.1 \%$ in men. The increase was most pronounced for the intermediate aggressive tumors from 3.3 in 1993 to 7.3 in 2010 with the largest increases for the adrenal gland, pancreas and lung. Conclusion: The incidence of NENs is increasing, but there are differences according to primary site, gender and tumor aggressiveness. Keywords: neuroendocrine, incidence, epidemiology, registry.

D4

\section{NETs in Hospital Registry of Russian Cancer Center}

Komarova L., Arion A., Patyutko Y., Gachramanov A.

N.N. Blokhin Russian Cancer Research Center, Moscow, Russia

Introduction: For the last $10-15$ years NETS are a large area of clinical and scientific investigation. Aim(s): To describe the frequency of NETs patients (pts), treatment and follow up of pts based on medical records in hospital archives. Materials and Methods: Retrospective observational study between 1990-2013 years. Results: Four hundred and eighty six pts with NETs were documented: GEP-NETs-264 pts, BNETs-149 pts. The most common tumor types were non-functional pancreatic tumors $-38.6 \%$. Primary tumors couldn't have determined in $6.2 \%$ of pts. The max increase in incidence for the last 10 years occurred for pancreas and bronchial 
NETS. We have detected 38 cases of small intestine NETs out of 486 pts. The median age for pancreatic NETs (139 pts) was 50.1 years; there was female preponderance (60.4\%). For the period 2007-2013 the pancreatic NETs accounts $2.4-2.9 \%$ of all pancreatic neoplasms in our clinic. Thirty six (29.5\%) had local, 8 (6.7\%) regional and 78 (64\%) distant disease at diagnosis. About $74 \%$ of pts underwent surgery. Metastases were detected in $31.4 \%$ most commonly in the liver and in $46.1 \%$ in the liver and other organs. The overall 1 year survival of the study population was $87.3 \%(95 \% \mathrm{Cl}$ [87.3-92.9]\%), 5 and 10 year survival were $67.6 \%(95 \% \mathrm{Cl}[49.6-81.5] \%)$ and $59.5 \%(95 \% \mathrm{Cl}$ $[26.0-86.5] \%)$. Conclusion: The study shows the relevant information regarding the prevalence of NETs in our Centre and current practices of management of patients in clinical departments. Keywords: nets, hospital registry.

\section{D5 \\ Survival and Predictors of Death for Patients with Bronchopulmonary Carcinoids in a Danish Tertiary NET Center}

\section{Kornerup L.S., Dam G., Grønbæk H. \\ Department of Medicine $V$, Aarhus University Hospital, Aarhus, Denmark}

Introduction: Bronchopulmonary carcinoids (BC) represent $1 \%$ of lung cancers and comprise typical (TC) and atypical carcinoids (AC). Aim(s): We present characteristics and mortality in all patients with TC and AC referred to a Danish tertiary NET Center between 2000-2014. Materials and Methods: Data was obtained from medical records, CT-scans, and pathology reports. Mortality was studied using Kaplan-Meier survival analysis. Results: We included 69 patients, 25 men and 44 women. 55 were diagnosed with TC (80\%) and 14 with AC (20\%). Mean age was 54 years for women and 57 years for men (NS). In patients with TC $60 \%$ were current/former smokers compared to $71 \%$ with AC (NS). $74 \%$ of TC patients and $79 \%$ of AC patients had non-metastatic disease at diagnosis (NS). Median follow-up was 5.8 years. Twelve patients died; 11 were smokers and 9 had a KI67 index $>2$. Among men $26 \%$ died compared to only $18 \%$ of women. $42 \%$ of patients with AC died compared to $15 \%$ with TC. Kaplan-Meier curves showed that metastatic disease, smoking and male gender had the strongest association with 5-year survival ( $\log$ rank, $\mathrm{P}=0.002, \mathrm{P}=0.03$ and 0.05 ), followed by KI67 index $>2(\mathrm{P}=0.11)$. No association with tumor type or $\mathrm{pCgA}$ levels. Conclusion: We found similar age and gender distribution as previously described in $\mathrm{BC}$. We observed that metastatic disease at diagnosis, smoking, and male gender are strong predictors of mortality within 5 years, while tumour type and $\mathrm{pCgA}$ levels were not associated with mortality. Keywords: bronchopulmonary carcinoid, typical, atypical, neuroendocrine, ki67 index.
D6

\section{Epidemiology and Classification of Gastroenteropancreatic Neuroendocrine Neoplasms Using the WHO 2010 Criteria: A Cohort Study in a Defined Norwegian Population}

Sandvik O. a, Søreide K. ${ }^{\text {, }}$ Kvaløy J.T. ${ }^{\mathrm{b}}$, Gudlaugsson E. ${ }^{\mathrm{a}}$, Søreide J.A. ${ }^{a}$

aStavanger University Hospital, Stavanger, Norway;

bUniversity of Stavanger, Stavanger, Norway

Introduction: Incidence figures of gastroenteropancreatic neuroendocrine neoplasia (GEP-NEN) still remain uncertain. $\operatorname{Aim}(\mathbf{s})$ : We evaluate the epidemiology of GEP-NEN in a well-defined Norwegian population. Materials and Methods: Patients diagnosed between 2003 and 2014 with GEP-NEN at Stavanger University Hospital, the only hospital for a population of 350000 , were included. A crosslinked search for appropriate diagnostic codes (ICD-9/10), morphology codes (SNOMED) and specific imaging (e.g. SRI) was used to identify eligible patients. Incidence rates were calculated by gender and age groups matched for population by Statistics Norway. Classification and staging was done according to the WHO 2010 criteria. Results: We identified 204 patients $(\mathrm{M}: \mathrm{F}=1.27: 1)$, with a median age of 61 (range 10-94) years. The annual overall crude incidence was 5.83/100.000 inhabitants. The adjusted overall incidence rate (Norwegian population) was 6.6/100 000; M 7.4 and F 5.8. Small intestine $(29.4 \%)$, appendix (23.5\%), pancreas (16.2\%) and rectum $(8.8 \%)$ were the most frequent tumour locations. Most gastrointestinal NENs were well-differentiated (59\% G1 and $21 \%$ G2, with $20 \%$ G3). The corresponding proportions for pancreatic NENs were $30 \%$, $47 \%$ and $23 \%$. UICC stage I was encountered in $38 \%$, stage II $8 \%$, stage III $18 \%$ and stage IV $35 \%$. In stage IV patients $76 \%$ had a G2 or G3 tumor. Conclusion: The incidence of GEP-NENs in southwestern Norway for the study period is among the highest reported. WHO grade correlates well with UICC stage group. Keywords: gepnen, epidemiology, who.

\section{D7 \\ European Information Network on Rare Cancers (RARECAREnet)}

Van der Zwan J.M. ${ }^{\text {, }}$ Trama A. ${ }^{\text {, }}$, Capoccacia R. ${ }^{\text {, }}$, Gatta G. ${ }^{\text {, }}$ Siesling $S{ }^{a}$

alKNL, Utrecht, The Netherlands; ' Instituto Nazionale dei Tumori, Milan, Italy; Instituto Superiore di Sanita, Rome, Italy

Introduction: NETs are considered as a rare cancer. Because of the complexity and the different disciplines involved, a multidisciplinary approach is desirable. The referral to centres of expertise $(\mathrm{CoE})$ is indicated as the optimal strategy for effective management. $\operatorname{Aim}(\mathbf{s})$ : Providing accurate information on rare diseases like NETS to all Eu citizens is clearly stated by the Eu Commission. In this perspective the Eu project called 'RARECARENet' is put in place. 
This project aims at building an information network for CoE and to provide comprehensive information for the community at large. Materials and Methods: During an expert meeting the ENETS criteria for $\mathrm{CoE}$ were followed to select criteria considered as feasible for cancer registries (CRs) to collect data on. A high resolution study is put in place, a data collection protocol and data entry tool was developed. Finally the data was checked for inconsistencies, this to ensure the quality of the data gathered. Results: CRs went back to the hospital files and pathological reports to collect additional data. The first results of this study performed in at least 4 countries are expected end 2014 and can presented at the conference. Differences between hospitals and the value of the indicators in their role of defining CoE will be presented. Conclusion: Information on stage, diagnosic- and therapeutic procedures were available but not always included in the standard datas. We concluded that only data on GEPNET $(n=2589)$ were of enough quality for analyses. Further conclusions will follow. Keywords: registry.

\section{Epidemiology/Natural History/ Prognosis - Prognosis}

\author{
E1 \\ Prognosis of Stage IV \\ Gastroenteropancreatic Neuroendocrine \\ Tumors (GEP-NET) According to the WHO \\ Classification and the Primary Tumor \\ Location \\ Carmona-Bayonas A. ${ }^{\mathrm{a}}$, Jiménez-Fonseca P. ${ }^{\mathrm{b}}$, Vieitez J.M. ${ }^{\mathrm{b}}$, \\ Menendez Prieto M.D. ${ }^{\mathrm{b}}$, Soli M.P. ${ }^{\mathrm{b}}$, Sanchez M.L. ${ }^{\mathrm{b}}$, \\ Rodriguez D. ${ }^{\mathrm{b}}$, Ruiz A.L. ${ }^{\mathrm{b}}$, Faez L. ${ }^{\mathrm{b}}$, Li W. $^{\mathrm{b}}$, Uriol E. ${ }^{\mathrm{b}}$, \\ Crespo G. ${ }^{c}$, Castaño A. ${ }^{\mathrm{d}}$, Garcia-Carbonero R. ${ }^{\mathrm{e}}$ \\ aDepartment of Oncology. Morales Meseguer University \\ Hospital, Murcia, Spain; 'bepartment of Oncology. \\ Asturias Central University Hospital, Oviedo, Spain; \\ 'Department of Oncology. Burgos University Hospital, \\ Burgos, Spain; dDepartment of Pathology. Fuenlabrada \\ University Hospital, Madrid, Spain; eDepartment of \\ Oncology. Virgen del Rocío University Hospital, Sevilla, \\ Spain
}

Introduction: WHO classification of GEP-NET is used to predict prognosis, yet survival within each grade shows wide variation. We hypothesized that primary tumor location may be partially responsible of this variability. Aim(s): To evaluate the prognosis of stage IV GEP-NET according to WHO classification and primary tumor location. Materials and Methods: We present a subset analysis from RGETNE (registry of Spanish NET Group (GETNE)). The impact of grade was assessed using Cox regression method. Covariates in the model were chromogranin, primary tumor size/location (gastrointestinal, pancreatic or unknown), functionality, ECOG PS and number of metastatic sites. gastrointestinal and grade 1 tumors were used as references categories. Results: 136 stage IV GEP-NET collected at a single center from 2005-2012 were analyzed. For each primary tumor location, the WHO grade was predictive of mortality. On Cox regression analysis, tumor grade was an independent predictor of survival [grade 2 (hazard ratio [HR], $1.63 ; 95 \% \mathrm{CI}, 1.05-2.52$ ); and grade 3 (HR, 4.65; 95\% CI, 2.71-7.97)]; as well as primary tumor location [pancreatic (HR, 0.69; 95\% CI, 0.45-1.06); unknown site (HR, 2.71; 95\% CI, 1.26-5.83)]. Bootstrap bias-corrected Harrell's C-index was 0.69 (95\% CI, 0.647-0.736) for tumor grade, and 0.71 (95\% CI, 0.657-0.753) for a model combining grade and location. Conclusion: The classification of GEP-NETs might be improved by incorporating covariates that reflect the underlying tumor biology, such as the primary site. Keywords: gep-net, who classification, grade, survival.

\section{E2}

\section{Follow-Up of $\leq 2 \mathrm{~cm}$ Non Functioning Pancreatic NETs in Patients with MEN1 Treated with Conservative Approach}

\author{
Davi M.V.a , Cosaro E. ${ }^{a}$, Malpaga A. ${ }^{\text {, }}$, Butturini G. ${ }^{\text {, }}$ \\ Ortolani S. ', Cingarlini S. c, Scarpa A. ', Francia G. ${ }^{a}$ \\ ${ }^{a}$ Medicine, Verona, Italy; 'bSurgery, Verona, Italy; \\ 'Oncology, Verona, Italy; ${ }^{\mathrm{d}}$ Pathology, Verona, Italy
}

Introduction: There is a current trend for conservative treatment of non functioning pancreatic NET (NF-PNETs), $\leq 2 \mathrm{~cm}$, in patients with MEN1, which are reported to be associated with low risk of metastasis and death. $\mathbf{A i m}(\mathbf{s})$ : The aim of our study was to asses the growth rate and occurrence of new NF-PNETs in a series of patients with MEN1 prospectively followed at University Hospital in Verona, Italy, between January 1998 and October 2014. Materials and Methods: Fourteen MEN1 patients with $\leq 2 \mathrm{~cm}$ NF-PNETs (median age at diagnosis 40.5 years, range 17-72) did not undergo surgery on the basis of institutional experience and literature. All patients performed abdomen CT or MRI and Octreoscan or Ga-68DOTATOC PET/CT, at diagnosis and CT or MRI during follow-up. Results: NF-PNET was single in 7 and multiple in 7 patients. The median size of the largest tumor was $16 \mathrm{~mm}$ (range 7-20 mm). The median follow-up after diagnosis was 4.5 years (range 1-16 yrs). In 10 patients $(71.4 \%)$ the size of tumors was stable. Two patients (14.2\%) were operated on because of an increase in tumor diameter after 1 and 7 years form PNET diagnosis. One or more new lesions $(\leq 1 \mathrm{~cm})$ was identified in 2 patients. Conclusion: The majority of MEN1 patients with NF-pNETs of $\leq 2 \mathrm{~cm}$ showed stable disease after a median follow up of 4.5 years while only 2 patients needed surgery for tumor progression. A conservative approach seems to be feasible for patients with small NF-PNETs which should be periodically followed with appropriate imaging. Keywords: pancreatic neuroendocrine tumor, men1. 
E3

\section{Gastric Neuroendocrine Neoplasias-Outcome Predictors-ENETS Staging and Grading System and Treatment}

\author{
Felder S. ${ }^{a}$, Jann H. ${ }^{\text {a }}$, Gerlach U. ${ }^{\text {, }}$ Pascher A. ${ }^{\text {, }}$ Denecke T. ${ }^{c}$, \\ Pschowski R. ${ }^{a}$, Prasad V. ${ }^{d}$, Arsenic R. ${ }^{e}$, Wiedenmann B. ${ }^{a}$, \\ Pavel M. ${ }^{\dagger}$ Pape U.F. \\ a Medizinische Klinik für Hepatologie und \\ Gastroenterologie (einschl. Arbeitsbereich \\ Stoffwechselerkrankungen), Charité-Universitätsmedizin,

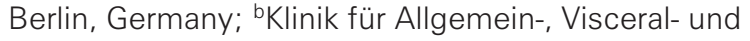 \\ Transplantationschirurgie, Charité-Universitätsmedizin, \\ Berlin, Germany; 'Klinik für Radiologie, Charité- \\ Universitätsmedizin, Berlin, Germany; ${ }^{d K}$ Kinik für \\ Nuklearmedizin, Charité-Universitätsmedizin, \\ Berlin, Germany; eInstitut für Pathologie, Charité- \\ Universitätsmedizin, Berlin, Germany; ${ }^{\dagger}$ Medizinische \\ Klinik für Hepatologie und Gastroenterologie (einschl. \\ Arbeitsbereich Stoffwechselerkrankungen), Charité- \\ Universitätsmedizin Berlin, Berlin, Germany
}

Introduction: Detailed analysis to guide management decisions in gNEN is rare. Aim(s): Characterize gNEN, identify prognostic factors, determine clinical outcome. Materials and Methods: Retrospective analysis of gNEN treated from 1988-2014. Descriptive and survival analysis with SPSS 19.0. Results: Of 141 cases 85 (61\%) were type-1, 7 (0.5\%) type-2, 24 (17\%) type-3 and $24(17 \%)$ type- 4 gNEN. 55\% were female, mean age at diagnosis was 58 years. WHO-grading was G1 in 49\%, G2 in 31\% and G3 in 20\%. cTNMstage was 0 in $18 \%$, I in $43 \%$, II in $12 \%$, III in $8 \%$ and IV in $20 \%$; pTNM staging (93\% of cTNM-stages) was comparable. While $1 / 85$ type- 1 was metastatic, there were 20/24 (42\%) stage IV in type- 3 and $15 / 24(63 \%)$ in type-4 gNEN. Median overall survival was significantly different between all types of gNEN (not reached in type-1, 8.9 in type- 3 and 2.9 years in type- $4 \mathrm{gNEN}$ ), between stages $0 / \mathrm{I}$, II/III and IV $(17.1,10.9,2.9$ years $)$ and between gNET-G1/2 and gNEC-G3 (3.0 years; $p<0.001)$. Resection, either surgical (SR, 43/33\%) or endoscopic (ER, 93/69\%), resulted in 5-\&10-year survival rates (YSR) of $62 \& 54 \%$ in SR and $89 \& 72 \%$ in ER with siginificantly better results in curative resections (CR, 5-YSR: SR 87\%). Chemotherapy (CTx) was frequently used in metastatic type-3 gNET (57\%) and type-4 gNEC (95\%) resulting in 2- and 5-YSR of 78 and $40 \%$. Conclusion: Our data support the prognostic role of clinicopathologic, WHO and TNM-staging of gNEN and the significant influence of both CR in early and palliative CTx in advanced disease. Keywords: nen, stomach, grading, tnm, outcome, surgery.

\section{E4}

Recurrent Disease after Curative Pancreatic Resection for Patients with Non-Functional Neuroendocrine Tumor; Identify the High Risk Patient

\author{
Jilesen A. ${ }^{a}$, Van Eijck $C .{ }^{b}$, Van Kemenade $F^{b}$,

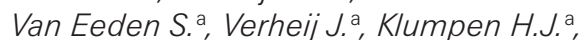 \\ Busch O. a, Bisschop P. , Dieren S. ${ }^{\text {, }}$ Gouma D. ${ }^{\text {, }}$ \\ Nieveen van Dijkum $E^{a}$ \\ academic Medical Center, Amsterdam, The Netherlands; \\ bErasmus Medical Center, Rotterdam, The Netherlands
}

Introduction: Since the overall favourable survival, detection of recurrent disease is one of the main goals during follow up in patients with resected pancreatic neuroendocrine tumors (pNET). Aim(s): The aim of this study was to analyse the risk for recurrent disease in an selective group of patients with non-functional pNET (NF-pNET). Materials and Methods: Retrospectively all resected NF-pNET from 1997-2013 of two academic institutions were included. Patients with distant metastases or hereditary syndromes were excluded. Pathology is revised according WHO classification 2010. Primary outcome was recurrent disease. Recurrent disease was defined as local tumor recurrence in the pancreas, localization in lymph nodes or distant metastases. An nomogram was made to predict recurrent disease within 5 year after curative resection. Results: Overall 107 patients were suitable for analysis and 63 had tumor grade 1, 36 tumor grade 2 and 8 patients had tumor grade 3 . After a median follow up of 52 months (IQR 29-81), 31 (30\%) patients developed recurrent disease of which 12 patients died due to tumor progression. Risk factors for recurrent disease were tumor location, tumor size $>2 \mathrm{~cm}$, tumor grade, positive lymph nodes, perineural and vascular invasion. Patients with a nomogram score $\geq 8$ were high risk patients for recurrent disease. The 5 and 10 year disease specific survival is respectively $91 \%$ and $77 \%$. Conclusion: High risk patients for recurrent disease should be monitored more closely and they may benefit from adjuvant treatment after resection. Keywords: nf.

\section{E5 \\ GastroEnteroPancreatic and Thoracic Neuroendocrine Tumors and the Ectopic Adrenocorticotropin Syndrome}

\author{
Kamp K., Alwani R.A., De Herder W., Feelders R.A. \\ Erasmus Medical Center, Rotterdam, The Netherlands
}

Introduction: Ectopic Adrenocorticotropin (ACTH) Syndrome (EAS) is associated with a variety of malignancies, predominantly of neuroendocrine origin. Several series report on the relative contribution of EAS in the spectrum of Cushing's syndrome. However, information on the incidence/prevalence and prognosis of EAS in the setting of patients diagnosed with Thoracic or GEP-NETs is virtually absent. Aim(s): Screening of a large consecutive series of thoracic and GEP-NET patients for the presence/occurrence of EAS in a retrospective case-record study in a tertiary academic referral center. 
Materials and Methods: 918 patients diagnosed with thoracic or GEP-NETs between 1993-2012 were studied. MEN1 \& SCLC were excluded. We differentiated between synchronous, metachronous and cyclic occurence of EAS. Synchronous EAS was defined as 6 months before and 6 months after first diagnosis of thoracic or GEP-NET. Overall survival was analyzed using a KM curve. Results: Of 918 patients with thoracic and GEP-NETs [469 males, 449 females; median age 58.7 years (range: $17.3-87.3)] 29$ patients $(3.2 \%)$ had EAS [10 males, 19 females; median age 48.1 years (range: 24.777.9)]. Synchronous EAS $(n=23)$, metachronous EAS $(n=4)$, cyclic $(n=2)$. NETs causing EAS: bronchial $(n=9)$, pancreatic $(n=9)$, thymic $(n=4)$, unknown $(n=4)$. Median OS: 41.4 months (range: $2.2-$ 250.9). Conclusion: The incidence of EAS in patients with thoracic and GEP-NETs is very low (3.2\%). Thoracic and Pancreatic NETs are the most common NETs causing EAS. Keywords: gep-net, thoracic, acth, cushing.

\section{E6}

\section{Appendiceal Goblet Cell Carcinoids: Prognostic Factors and Selection of the Most Appropriate Adjuvant Management in a Retrospective Series}

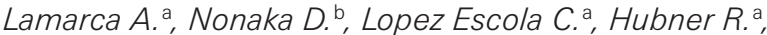 \\ Fulford P. ${ }^{\mathrm{c}}$, Chakrabarty B. ${ }^{\mathrm{b}}$, Valle J. ${ }^{\mathrm{a}}$ \\ The Christie NHS Foundation Trust: Medical Oncology, \\ Manchester, UK; ${ }^{b}$ The Christie NHS Foundation Trust: \\ Pathology, Manchester, UK; ${ }^{c}$ The Christie NHS Foundation \\ Trust: Surgery, Manchester, UK
}

Introduction: Appendiceal goblet cell carcinoids (GCC) are rare tumours with low-quality data regarding its management $\operatorname{Aim}(\mathbf{s})$ : Identification of prognostic factors. Materials and Methods: Patients (pts) with GCC at our institution (1996-2014) were reviewed for overall survival (OS, primary end-point), disease-(DFS)/progression-free survival (PFS) and relapse rate (RR). Results: Seventyfour of $97 \mathrm{pts}$ were eligible after pathology review (median follow-up 27.6 mo (range 3.8-217.3)): 54\% female: median age 56.4 yrs (range 25.9-83.1); stage I (3\%), II (39\%), III (16\%), IV (38\%); Tang group A $(45 \%)$, B $(42 \%)$, C $(7 \%)$. Most pts $(56 ; 76 \%)$ were treated with curative intent $(\mathrm{CI})$ (surgery \pm cytoreductive surgery followed by hyperthermic intraperitoneal chemotherapy (HIPEC) \pm adjuvant chemotherapy; $36 \%, 20 \%$ and $9 \%$ pts treated with CI received adjuvant HIPEC, chemotherapy or both, respectively; $23 \%$ received palliative treatment (PT) (chemotherapy or best supportive care) only. Median OS (all pts), DFS (CI pts) and PFS (PT pts) was 52.1 (95\% CI 29.490.3), 75.9 (26.6-NR) and $5.3(0.6-5.7)$ mo. There was no difference in OS $(\mathrm{p}=0.07)$ between the adjuvant subgroups; $p$ ts with HIPEC had a lower RR $(p=0.001)$. Stage $(p<0.001)$ and age $(p=0.032)$ were independent prognostic factors for OS; Tang showed a trend for OS ( $\mathrm{p}=0.059$, not statistically significant likely due to a lack of power). Conclusion: Stage and Tang are confirmed prognostic factors for OS; the optimal adjuvant approach remains unclear - prospective studies are needed. Keywords: goblet cell, appendix.
E7

'Wait and Watch' Approach for Small Pancreatic Neuroendocrine Tumors (pNETs): Prognosis and Survival in 51 Consecutive Patients

Massironi S., Rossi R.E., Zilli A., Conte D., Ciafardini C., Peracchi $M$.

1Fondazione IRCCS Ca' Granda Ospedale Maggiore Policlinico, Gastroenterology and Endoscopy Unit, Milan, Italy

Introduction: The most appropriate management for small nonfunctioning pancreatic neuroendocrine tumors (pNETs) is lacking and whether all these lesions should be routinely resected is still debatable. Aim(s): Endpoints of the study were the overall survival (OS) and the progression free survival (PFS) of pNETs, according to their clinical management. Materials and Methods: From 2009 to 2014 51 patients were newly diagnosed as having pNET. Among them, 15 patients with small $(\leq 20 \mathrm{~mm})$ pNETs underwent a 3-month follow-up for the first year and then biannual (FU). TNM stage was I in all but one patient (stage IIA). 21 patients underwent surgical resection (SR): TNM stage was I, IIA, IIIB, IV in two, nine, one, nine cases, respectively. 15 patients received systemic therapy (ST) due to advanced disease or contraindications to surgery; of them five, two and eight were at stage IIA; IIB and IV, respectively. Results: Median followup of the entire cohort was 56 months. OS was similar in FU and SR groups, whilst it was significantly worst in ST subset (log-rank test $\mathrm{P}=0.014$ ). Five-year survival rate was $100 \%$ in the FU group, $90 \%$ in the SR, and $61 \%$ in the ST group $(p<0.0001)$. PFS did not differ in the three groups as disease remained stable in all but one patient in the FU group, whilst six patients (28\%) in the SR group and five (31\%) in the ST group showed disease progression. Conclusion: The "wait and watch" approach appears to be safe in early stage pNETs, though larger and prospective studies are needed. Keywords: pancreatic neuroendocrine tumor, prognosis.

\section{E8}

\section{Years of Experience in a Single Centre on Neuroendocrine Pancreatico-Duodenal Tumors in Multiple Endocrine Neoplasia Type 1 Syndrome}

\author{
Milanetto A.C. ${ }^{\text {a }}$ Lico V. ${ }^{\mathrm{a}}$, Alaggio R. ${ }^{\mathrm{b}}$, Martini C. ${ }^{\mathrm{c}}$, \\ Pedrazzoli S. ${ }^{a}$, Pasquali $C{ }^{\text {a }}$ \\ aPancreatic and Digestive Endocrine Surgical Unit, Padua,

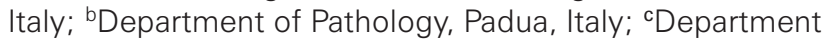 \\ of Endocrinology, Padua, Italy
}

Introduction: Multiple Endocrine Neoplasia type 1 (MEN1) syndrome has an autosomal dominant transmission. Combination of tumors: parathyroid, pituitary and pancreatico-duodenal (PD) NETs. PD NETs in MEN1 syndrome show propensity for malignant degeneration. Aim(s): We evaluated occurrence of PD, therapeutic strategies and follow up (FU) of PD NETs in MEN1 patients observed in 
our Unit. Materials and Methods: From 1975 to 2013: 322 PD NETs, 40/322 had a MEN1 syndrome. Average 40.5 years (range 13-69 yrs), $20 \mathrm{M} / 20 \mathrm{~F}$. Only patients with MEN1 syndrome and a PD NET diagnosed by imaging or histology were included. FU to December 2013. Results: Among the 40 MEN1 patients: 20 nonfunctioning (NF) NETs, 16 Zollinger-Ellison Syndrome, 3 insulin secretin tumors. All but one MEN 1 patients had hyperparathyroidism, $40 \%$ pituitary tumors, $25 \%$ adrenal involvement, $20 \%$ carcinoids. 11/40 malignant disease: 5 liver metastases, and 6 as late onset after a median time of 7 years. Only 4/11 died for disease related cause (2 disease progression). 23/40 PD disease as first involvement of MEN1 syndrome. 29/40 underwent surgical resection of the primary PD NET, $3 / 29$ with associated liver metastasectomy. FU: 32 alive (median FU 9 yrs; range 0.5-29 yrs). 8 deaths: 7 for disease related causes, 1 for other unrelated disease (median FU 9.5 yrs; range 0.5-28 yrs). Conclusion: Most MEN1 patients have as first involvement a PD disease (57.5\%). Patients die for in/direct effects of desease. Distant metastases occur up to $29 \mathrm{yrs}$ from diagnosis. Keywords: net, men1 syndrome, pancreatic tumor.

\section{E9}

\section{Describing NENs: Just Clinical/Biologic Features or Actual Prognostic Factors?}

Ribeiro C., Gomes F., Semedo M., Filipe F., Oliveira S., Barata P., Custodio P., Costa L., Sousa M., Mascarenhas L., Luz R., Batarda L.

CHLC, Lisbon, Portugal

Introduction: Neuroendocrine neoplasias (NENs) are a rare group of diseases, with several possible locations and manifestations, often representing a challenging diagnosis. It is essential to improve tools that accurately predict aggressiveness and prognosis. Aim(s): To assess differences in prognosis of gastrointestinal NENs according to location, histology and grade. Materials and Methods: Retrospective analysis of clinical records of patients with NENs, during 10 years in Centro Hospitalar de Lisboa (CHLC). Results: We reviewed 86 cases of NENs diagnosed between Jan 2004 and Jan 2013. 47.9\% were females. The median age was 70 years $(12,100) .75 \%$ were NETs, $17 \%$ MANETs and $8 \%$ were NECs. Differentiation grade distributed as follows: $48 \% \mathrm{G} 1,32 \% \mathrm{G} 2$ and $20 \%$ G3. Regarding location, colon was the most common $(25 \%)$, followed by stomach $(23 \%)$, small bowel $(21 \%)$, rectum $(19 \%)$ and pancreas (12\%). The median follow up time was 75.3 months (25.1, $133.4)$, with a median survival of 45.6 months $(0,133.4)$. To date, $60.4 \%$ of patients had died, with a median survival of 12.9 months $(0.1,100.5)$ in this group. Apart from metastatic disease, no other feature was associated with survival on the univariate analylis. However, on a multivariate model, age, histological subtype, location, neuroinvasion, grade, Ki67 and interation of metastatic disease/surgery were all significantly associated with prognosis. Conclusion: Though a small sample, this results may be important orienting larger, prospective studies. Keywords: neuroendocrine neoplasias, prognosis.

\section{E10 \\ Ki67 as a Prognostic Marker in Neuroendocrine Tumours: A Systematic Review of the Literature and Quantitative Synthesis of 5 Year Survival Data}

\author{
Richards-Taylor S., Tilley C., Hu J.H., Pearce N., \\ Armstrong T., Jaynes E., Nolan L., Cave J. \\ University Hospital Southampton NHS Foundation Trust, \\ Southampton, UK
}

Introduction: The ENETS guidelines classify Gastroenteropancreatic Neuroendocrine Tumours (GEP NETs) according to the histological assessment of tumour mitotic count or Ki67 labelling index (LI) on paraffin-embedded tissue, because proliferation rate has been shown to strongly correlate with prognosis. Since the guidelines were published, Ki67 has been established as the more reproducible measure, and further large case series have been reported. Aim(s): To systematically review articles which report the prognosis of patients by Ki67 LI. The aim of this study is to improve the ability of clinicians to prognosticate for their patients. Materials and Methods: Medline was searched using the terms 'neuroendocrine' AND 'tumors OR tumours OR tumor OR tumour' AND 'Ki67 OR Ki67 OR mib1 OR mib-1' AND 'prognosis OR survival OR mortality'. 265 abstracts were identified. Articles were excluded if they described non-human specimens, were non-English language, or were published prior to 2000. Abstracts were reviewed to include only papers that related Ki67 and survival outcome, and exclude those reporting non GEP NETs, sub-groups selected by treatment modality, and small series ( $<20$ cases). 11 papers were identified and included. Results: Published 5 year survival rates will be summarised, with additional data from our own institution included. Conclusion: Clear guidelines regarding grade have facilitated direct comparisons between case series. There is now a substantial body of evidence linking ki67 and survival. Keywords: gep-net, prognosis, ki67.

\section{E11 \\ Comparative Marker Assessment in Small Cell Lung Cancer with Good and Poor Prognosis \\ Schmidt L. a, Specht E. ${ }^{a}$, Reimann C. ${ }^{a}$, Kaemmerer D. ${ }^{\mathrm{b}}$,

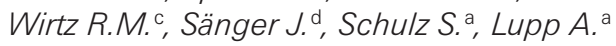 \\ aDepartment of Pharmacology and Toxicology, Jena University Hospital, Jena, Germany; 'bepartment of General and Visceral Surgery, Zentralklinik Bad Berka, Bad Berka, Germany; 'Stratifyer Molecular Pathology, Köln, Germany; 'Laboratory of Pathology and Cytology, Bad Berka, Germany}

Introduction: Small cell lung cancers (SCLC) are highly aggressive and poorly differentiated neuroendocrine neoplasms which comprise $\sim 15 \%$ of all lung malignancies. $\operatorname{Aim}(\mathbf{s})$ : In a panel of patients with diagnosed SCLC with poor and good prognosis we investigated the prognostic impact by analyzing protein and mRNA 
expression levels of 9 molecular markers. Furthermore we studied the influence of therapeutic interventions on the prognosis. Materials and Methods: Formalin-fixed, paraffin-embedded tumor samples of 27 SCLC patients with good prognosis (survival $\geq 30$ months) and 19 patients with poor prognosis (survival $<30$ months) were analyzed for the receptors SSTR1, 2A, 3, 4, 5, CXCR4 and the proliferation markers Ki-67, TOP2A and RacGAP1 by means of immunohistochemistry and RT-qPCR. Results: SSTR2A, 5 and CXCR4 were expressed most frequently. High levels of all proliferation markers were observed. In case of CXCR4 and RacGAP1 different expression levels could be shown in dependence on the survival. The combination of chemotherapy, radiation and surgery is associated with the best prognosis. Conclusion: The high expression levels of SSTR2A, 5 and CXCR4 in SCLC open up the possibility for targeted diagnostics and therapies. High proliferation rates suggest the use of antiproliferative agents, not least because of the positive outcome of the patients treated with the combination therapy. In addition, two markers showed prognostic influence. Keywords: sclc, sstr, cxcr4, proliferation.

\section{E12}

\section{Management and Clinical Outcome of Patients with MEN 1 Disease Presenting Non Functioning Pancreatic Neuroendocrine Neoplasms (NF-pNEN) $\leq \mathbf{2} \mathbf{c m}$}

\author{
Tamburrino D. ${ }^{a}$, Partelli S. ${ }^{\mathrm{b}}$, Pasquali C. ${ }^{\mathrm{c}}$, Bartsch D. , \\ Valente R. ${ }^{a}$, Milanetto A.C. ${ }^{c}$, Albers M. ${ }^{\text {, }}$ Toumpanakis C. , \\ Lopez C. ${ }^{\text {, }}$ Fusai G. ${ }^{a}$, Caplin M. ${ }^{\text {a }}$ Falconi M. ${ }^{\mathrm{b}}$ \\ aRoyal Free Hospital, London, UK; bUniversity Hospital \\ Ancona, Ancona, Italy; 'University of Padua, Padua, Italy; \\ dUniversity of Marburg, Marburg, Germany
}

Introduction: The role of surgery for NF-pNEN $\leq 2 \mathrm{~cm}$ in MEN1 patients is still controversial. Aim(s): To evaluate outcomes of patients affected by MEN1 syndrome with NF-PNET $\leq 2 \mathrm{~cm}$. Materials and Methods: This was a retrospective multicenter study that involved 4 European institutions. All MEN1 patients with NF-pNEN $\leq 2 \mathrm{~cm}$ were enrolled. Results: Overall, 61 patients were included. Of those, 27 patients (44\%) were treated conservatively at diagnosis whereas the remaining 34 patients (56\%) underwent upfront surgery. Among 27 patients conservatively managed, 9 patients underwent surgical resection during follow-up. At a median follow-up of 50 months, all patients conservatively treated did not develop metastases during follow-up. Three patients (5\%) developed liver and nodal metastases. All of these patients previously underwent pancreatic resection. Only one patient died after surgical resection for surgery-related complications. Disease-specific mortality was nil. Conclusion: NF-pNEN $\leq 2 \mathrm{~cm}$ in MEN1 syndrome is an indolent disease. A conservative approach for these forms is feasible and oncologically safe. Keywords: men1 syndrome, pancreatic neuroendocrine tumor, treatment.

\section{Epidemiology/Natural History/ Prognosis - Descriptive Epidemiology}

\author{
F1 \\ Ectopic Cushing's Syndrome (ECS) in \\ Patients with Neuroendocrine Neoplasms \\ Alexandraki K., Boutzios G., Papaoikonomou E., \\ Chatzellis E., Chrysochoou M., Tsoli M., Kaltsatou M., \\ Nikolopoulos G., Moschouris P., Bouki A.E., Kaltsas G. \\ Endocrine Oncology Unit, Section of Endocrinology, \\ Department of Pathophysiology, National and \\ Kapodestrian University of Athens Medical School, \\ Athens, Greece
}

Introduction: Ectopic Cushing syndrome (ECS) has been described in patients with neuroendocrine neoplasms (NENs). $\operatorname{Aim}(\mathbf{s})$ : The prevalence of ECS in NENs has not been extensively studied. Materials and Methods: We evaluated 152 patients (69 females, mean age $49 \pm 17$ yrs) with NENs: 10 gastric, 9 duodenal, 65 pancreatic, 13 small intestinal, 25 appendiceal, 5 colonal, 9 lung, 1 thymic, 9 unknown primary origin (UPO), 4 other; 2 harboured 2 NENs simultaneously. Results: 36 (24\%) patients had sporadic functioning NEN (11 carcinoid syndrome, 8 gastrinoma, 7 insulinoma, 6 ECS, 1 VIPoma, 1 ectopic GHRH, 1 PTH-rP, 1 5HIAA secretion) and 116 non-functioning NENs. 6 patients $(54 \pm 18 \mathrm{yrs}, 4$ males, $4 \%$ of total and 17\% among functioning NENs) had ECS: 2 atypical lung (stage 1), 1 atypical thymic (stage 4), 1 UPO (stage 4, grade 2), 2 females with typical lung (stage 3, stage 1 , respectively). Among the 9 patients with lung NENs the prevalence of ECS was $44 \%$ or $13 \%$ among UPO NENs. 5/6 neoplasms originated from the foregut. 1 patient had subtle disease, 5 had overt CS. The patient with thymic NEN succumbed 3.5 years after diagnosis; 4 patients with lung NENs and 1 with UPO NEN are still alive with good control of hypercortisolemia and mean follow-up $62 \pm 42$ months. Conclusion: $4 \%$ of NENs patients developed ECS that rose to $25 \%$ among foregut and $44 \%$ among lung NENs. Although this is a relatively small series influenced by referral bias these findings could suggests that patients with foregut or more particularly lung NENs should be evaluated for subtle or overt CS. Keywords: ecs. 


\section{F2}

\section{Pancreatic Neuroendocrine Tumors in Denmark and Introduction of Cancer Package Pathways}

\author{
Christensen C.U., Grønbæk H. \\ Department of Hepato - and Gastroenterology, Aarhus \\ University Hospital, Aarhus, Denmark
}

Introduction: New referral procedures were introduced into the Danish healthcare system on Jan 1, 2009 in malignant diseases. Cancer-package pathways purposed to streamline diagnostics and treatment specifically to reduce the waiting time from diagnosis to initiation of specialized treatment. Aim(s): This study aimed to determine if time between diagnosis and first outpatient visit is reduced under the new directives. Materials and Methods: PNET patients treated in our center from 1993 to August 20, $2014(\mathrm{n}=92)$ were included and stratified with diagnosis before or after January 1 st, 2009. Primary endpoint was time between histopathological diagnosis and first visit post-diagnosis. We used Students t-test for group comparison and Kaplan-Meier curves and log-Rank test for survival analysis. Results: 53 patients before and 39 after January 1st, 2009 were included correlating with incidenses of 0.17 and $0.36 / 100.000 /$ year, respectively. Mean duration between diagnosis and first visit was 59 (95\% CI: 37-92) days in the group diagnosed before 2009 and 21 (95\% CI: 15-28) days in the group diagnosed from 2009-2014; the duration was reduced by 38 days after introduction of cancerpackage pathways $(\mathrm{p}<0.001)$. 2-year survival was insignificantly increased from $68 \%$ before 2009 to $84 \%$ with diagnosis after 2009 $(p=0.19)$. Conclusion: PNET incidence is increasing. Cancer package pathways and diagnosis and treatment centralization are beneficial for patient flow. Further, cancer package pathways correlate with improved survival. Keywords: pnet, incidence, referral, delay.

\section{F3}

\section{Trends in Neuroendocrine Tumor in Friuli Venezia Giulia in the Last 20 Years}

\author{
Grimaldi F., Cipri C., Motta C., Pellegrini M.A., Piussi S., \\ Purinan A., Tonutti L., Vescini F. \\ Endocrinology and Metabolism Unit, Udine, Italy
}

Introduction: Epidemiological study conducted on patients (pts) with neuroendocrine tumors (NEN) at the regional referral center for Endocrinology and Metabolic Diseases of the Udine Hospital, Italy. Aim(s): Data collected on 344 pts between 1990-2013 on residence, NEN type, age and metastases at diagnosis, survival, clinical course length, diagnostic tools, specialist visits, therapy. Survival and progression-free survival (PFS) analyzed by sex, NEN type, metastases at diagnosis. Materials and Methods: Mean age at diagnosis was 59.8 yrs (19-87 yrs), 50.3\% males. Lungs and pancreas most affected; primarily non-functioning of the pancreas and typical carcinoid. Diagnoses of NEN/yr increased gradually to $>30$ cases $/ y r$ from 2009 on, greater in GEP-NEN. Between 2003-2013 there were 19.7 cases/100000 inhabitants; $20.8 \%$ had liver metastases at diagnosis.
Survival was 1 mo - 23.7 yrs; $31.6 \%$ of pts died, $71.1 \%$ due to NEN. Results: Comparing the clinical course of pts seen before 2006 (year a Network started training the general practitioners on NEN) with those afterwards, $44.9 \%$ saw an endocrinologist within 3 mos. of diagnosis vs. $68.8 \%$. Computed Tomography was the prevalent test (63.1\%) for diagnosis; $44.5 \%$ of pts had no therapy; of the rest, $40.7 \%$ had somatostatin analogues. Survival and PFS were higher in women, in pts without liver metastases at diagnosis and those with typical carcinoid who had the longest survival time. Conclusion: Data confirm the increased NEN incidence in our Region and provide information on the NEN epidemiology. Keywords: survival.

\section{F4}

\section{The Clinical Oncology Society of Australia (COSA) SIGNETURe Neuroendocrine Tumor (NET) Registry of Australia: First Report of Patient Characteristics and Patterns of Care}

Price T. ${ }^{a}$, Pavlakis N. ${ }^{\mathrm{b}}$, Karapetis C. ${ }^{\mathrm{C}}$, Michael M. ,

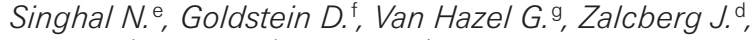
Hicks R. ${ }^{\text {, }}$ Yeend S. ', Chua Y.J.'

aThe Queen Elizabeth Hospital and University of Adelaide, Adelaide, Australia; 'boyal North Shore Hospital, Sydney, Australia; 'Flinders Medical Centre, Adelaide, Australia; 'Peter MacCallum Cancer Institute, Melbourne, Australia; eRoyal Adelaide Hospital, Adelaide, Australia; fPrince of Wales Hospital, Sydney, Australia; '9Sir Charles Gardiner

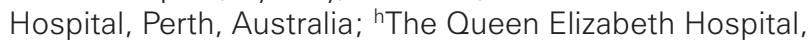
Canberra, Australia; 'Canberra Hospital, Canberra, Australia

Introduction: NETs remain a relatively rare malignancy. Clinical trials are often hindered by the limited number of patients available and thus registries provide important information about patterns of care and patient outcomes. $\operatorname{Aim}(\mathbf{s})$ : With this in mind the SIGNETURe registry was developed to record patient characteristics, therapies used and outcomes for patients with NETs in Australia. Materials and Methods: Patients seen at specialist NET referral centres were offered participation in the registry. Both newly diagnosed cases (incident cases) and those with a prior established diagnosis (prevalent cases) were included. The registry was reviewed by an appropriate Ethics committee in each state of Australia. An electronic web based database was used for data collection (DATATRAKC). Results: From 9/2009 until 9/2014, 228 patients were enrolled from 9 centres. The median age 65 years (range $18-87$ years). 57\% were male. Sites of disease were as follows; $24 \%$ ileum to distal jejunum, $22 \%$ pancreas, $15.4 \%$ unknown primary, $7 \%$ colon or rectum, $5.3 \%$ appendix and $5.3 \%$ gastric. There was one anal and ovarian primary respectively. The primary had not been entered in $21 \%$ of cases. Distribution of primary was not significantly altered by sex. Reporting of mitotic rate was as follows, $<221.5 \%, 3-2019 \%,>20$ $1.5 \%$. For $58 \%$ of cases the mitotic rate was entered as unknown. $63 \%$ had advanced disease. Conclusion: These first data describe a cross section of NET patients referred to centres with NET interest in Australia. There remain patients who are n. Keywords: registry. 
F5

\section{Risk and Protective Factors for Midgut Carcinoid Tumours: A Case-Control Study of Prospectively Evaluated Patients}

\author{
Rinzivillo M. a, Capurso G. a, Campana D.b, Fazio N.c, \\ Panzuto F., Spada F. ${ }^{\mathrm{a}}$. Cicchese N. ${ }^{\mathrm{a}}$, Tomassetti P. \\ Falconi M. ${ }^{d}$, Delle Fave . $^{\text {a }}$ \\ aDigestive and Liver Disease Unit, Sapienza University \\ of Rome, Rome, Italy; bepartment of Internal Medicine \\ and Gastroenterology, University of Bologna, Bologna, \\ Italy; 'IEO, European Institute of Oncology, Milan, Italy; \\ dSurgical Pancreas Unit, Università Politecnica delle \\ Marche, Ancona, Italy
}

Introduction: The incidence of midgut carcinoid tumours is on the rise, but few studies have investigated risk factors for their occurrence. Aim(s): We therefore aimed at clarifying risk factors for the occurrence of sporadic midgut carcinoid tumours. Materials and Methods: Multicentre case-control (C-C) study. Patients prospectively evaluated at partecipating centres. Controls with non-neoplastic/non-chronic disorders seen at GI outpatients clinics, matched (4:1) for sex and age. Results: 215 MCTs and 860 controls enrolled. At the multivariate analysis, fh of colorectal cancer (OR 1.70; 95\% $1.01-3.02, \mathrm{p}=0.05$ ), fh of breast cancer (OR 2.23; 95\% CI $1.27-$ $3.91, \mathrm{p}=0.008)$ and heavy smoking (OR 2.14; $95 \%$ CI $1.44-3.19$, $\mathrm{p}=0.0001$ ), were associated with an increased risk of carcinoid occurence, while the use of aspirin resulted a protective factor (OR $0.19 ; 95 \%$ CI $0.05-0.65, p=0.007)$. Conclusion: Family history of colorectal and breast cancer, and heavy smoking are risk factors for the development of midgut carcinoids, while aspirin is a protective factor. These factors are similar to some of these associated with colorectal cancer, thus suggesting a possible overlap with risk factors for exocrine intestinal carcinogenesis. Moreover, the elucidated risk factors for MCTs are different from those previously associated with pancreatic neuroendocrine tumours (family history of PDAC, high alcohol intake, recent onset diabetes, chronic pancreatitis) underlining different mechanisms of carcinogenesis for endocrine cells in different sites. Keywords: midgut carcinoid tumours.

F6

\section{Cardiac Evaluation as Part of the Initial Extension Studies in Neuroendocrine Tumors. Experience in a Multidisciplinary Team. ARGENTUM Group}

\author{
Santos D. ${ }^{\mathrm{a}}$ O'Connor J.M. ${ }^{\mathrm{a}}$, Pesce V. ${ }^{\mathrm{b}}$, Bestani C. ${ }^{\mathrm{b}}$, Belli S. ${ }^{\mathrm{a}}$, \\ Domenichini E. ${ }^{a}$, Marmissolle F. ${ }^{\mathrm{c}}$, Roca E. ${ }^{\mathrm{b}}$ \\ alexander Fleming Institute, CABA, Argentina; \\ ${ }^{\mathrm{b}}$ Gastroenterology Hospital B. Udaondo, CABA, Argentina; \\ cSITE, La Plata, La Plata, Argentina
}

Introduction: Carcinoid heart disease (CHD) is a complication of metastatic neuroendocrine tumors and is screened for using echocardiography. Published case series showed that when cardiological evaluation is done at least $50 \%$ of the patients have CHD, and $33 \%$ had surgical indication. Aim(s): To describe clinical cardiological visit and echocardiographic assessment as a screening at the time of diagnosis of carcinoid syndrome (CS) in our Group. Materials and Methods: 28 patients were referred to the Department of cardio-oncology at the diagnosis of carcinoid syndrome (CS) for a prospective evaluation. Population characteristics were described. The endpoint was time to surgical indication and the differences between causes of A) dead during follow up and B) alive during follow up. Cardiac involvement was evaluated by clinical symptoms and echocardiographic findings. Results: Patients with CHD: 28. During follow up: 8 had events (deaths). 4 cardiac: 1 with surgery, 3 under medical treatment, 4 because of disease progression. Median follow up was 37 months (6-78) Median age in the group of patients with events (death) was 65 years. Higher value of serum $\mathrm{CgA}$ in patients with events, $337 \mathrm{~nm} / 1$ compares with alive patients, $258 \mathrm{nmol} / 1$ p 0.004 . Score was higher in a first group of patient 12 versus 6 . Conclusion: Cardiac evaluation is an important part of the followup in carcinoid tumors. Medical treatment is important in prevent and delay clinical course of the disease (CHD). Keywords: cardiac heart disease, neuroendocrine tumors.

F7

Preliminary Results on the Diagnosis of NETs in Portugal - The Cross Sectional TNE-ETC Survey

Santos A.P., Bugalho M.J. ${ }^{b}$, Lopes J.M. ${ }^{c}$, Claro I. ${ }^{\text {, }}$

Sanches A.C. ${ }^{a}$, Gomes L. ', Catarino A.L. ${ }^{\text {, }}$ Preto J. ${ }^{\dagger}$, Fernandes I. ${ }^{\mathrm{g}}$, Pereira B. ${ }^{\mathrm{h}}$, Marques A.P., Rodrigues F.

alPOFG-Porto, Porto, Portugal; ' bIPOFG-Lisboa, Lisboa, Portugal; ' $I P A T I M U P / H S J$, Porto, Portugal; ${ }^{d} \mathrm{CHUC}$, Coimbra, Portugal; eHBA/H.Luz, Lisboa, Portugal; 'HSJ, Porto, Portugal; ${ }^{9} \mathrm{CHLN}$, Lisboa, Portugal; ${ }^{\mathrm{h}} \mathrm{HGO}$, Almada, Portugal; 'ULS Matosinhos, Matosinhos, Portugal; 'JPOFGCoimbra, Coimbra, Portugal

Introduction: NETs are increasing worldwide. Epidemiological data is lacking in Portugal. The NETs Study Group of the Portuguese Endocrine Society is committed to develop a National Registry. $\operatorname{Aim}(\mathbf{s})$ : To perform a multicenter cross-sectional study, to characterize demographic and clinical features of digestive NETs in Portugal. Materials and Methods: Cases identified from 15 participating centers over a period of 18 months; histological diagnosis mandatory for inclusion. Data collected in an electronic platform using PHP and JavaScript technologies, stored in a MySQL database. Demographic, clinical, histopathological and diagnostic data collected. Ethical principles concerning national legislation fulfilled. Statistical analysis performed with SPSSv15.0. Results: 314 pts identified; 21 excluded due to absent inclusion criteria; 293 analysed (age: $56.6 \pm 13.6 \mathrm{y} ; \mathrm{F} / \mathrm{M}$ : $54.6 / 45.4 \%), 3$ hereditary syndromes. Main primary sites: pancreas (31.1\%), ileum (21.1\%) and stomach (13.7\%); 2\% unknown; grading: NETG1 (55.6\%), NETG2 (19.5\%) and NEC (9.9\%), 81\% with immunohistochemistry. There was a trend for older age at diagnosis of NEC and association between grading and staging. Octreoscan ${ }^{\circledR}$ $(37.5 \%)$ and $\mathrm{CT}(71.4 \%)$ were the main staging techniques. At diag- 
nosis LN (37.2\%); distant mets (29.4\%), mainly the liver (33.7\%). Conclusion: Our findings fit with reports of NETs from other countries. These preliminary results reinforce the need of national clinical framework and reference centres to improve diagnosis, clinical management and outcome of NET patients in Portugal. Keywords: Portugal, net.

\section{F8}

\section{Epidemiologic Analysis of Neuroendocrine Tumors in Cancer Center: Tianjin, China}

\author{
Wang J. ${ }^{a}$, Sun Y.S. ${ }^{\text {, }}$ Wang X. ${ }^{\text {a }}$, Hao J. ${ }^{a}$ \\ aDepartment of Pancreatic cancer, Cancer Hospital of \\ Tianjin Medical University, Tianjin, China; 'Pathology \\ Department, Cancer Hospital of Tianjin Medical University, \\ Tianjin, China
}

Introduction: An increased incidence of neuroendocrine tumors (NETs) has been reported worldwide. $\operatorname{Aim}(\mathbf{s})$ : As the third biggest cancer center in China, we here report the epidemiologic characteristics of NETs in our hospital. Materials and Methods: A retrospective cohort study was conducted at cancer Hospital of Tianjin Medical University. Patients with a NET diagnosis from Jan-2000 to Jun-2014 were included. Results: Totally, nine hundred fifty-seven NET cases were identified. The overall incidence of NETs increased from 0.06 (2000), 0.58 (2010) to 1.37 (2013) per 100,000 per year. The incidence is increasing at a rate of $10 \%$ per year. All the cases include $61.2 \%$ male with average age 56.1 and $38.8 \%$ female with average age 51.8. NETs occur most frequently in bronchopulmonary system $(25.2 \%)$ with the second most common location in pancreas $(21.0 \%)$, followed by gastric $(18.0 \%)$, rectum $(12.1 \%)$, colon and appendix $(6.6 \%)$, intestine $(2.1 \%)$, ovaries $(1.2 \%)$ and hepato-biliary system (1.1\%). Given the overall incidence of the disease, the prevalence of GEP-NETs is the most common. All cases were separated into G1 $(17.9 \%), \mathrm{G} 2(8.6 \%)$ and $\mathrm{G} 3(73.6 \%)$ depending on the prognostic value of $\mathrm{Ki}-67 \%$ and mitotic count. G1-G2 occur most frequently in pancreas. Metastases were found in $36.9 \%$ after the initial diagnosis. Conclusion: The incidence of NETs has markedly increased over the course of 13 years. This is the first study to provide epidemiology characteristics of Chinese. Keywords: neuroendocrine tumors, epidemiology, china.
F9

\section{Epidemiological Characteristics from a Single Centre Database. Cohort of 1301 Neuroendocrine Tumor Patients: Is There an Association between Age and Grade of NET? \\ Yalchin M., Garcia-Hernandez J., Mohmaduvesh M., Toumpanakis C., Caplin $M$. \\ Neuroendocrine Tumour Unit, ENETS Centre of \\ Excellence, Royal Free Hospital, London, UK}

Introduction: There is limited data related to epidemiology of NETs and patient survival. For many cancers younger age of diagnosis can be associated with worse survival. $\operatorname{Aim}(\mathbf{s})$ : To provide a breakdown of the different characteristics of NET presenting to a tertiary referral centre and examine the association between age of diagnosis and survival. Materials and Methods: Retrospective analysis of 1301 NETs from the database (2000-2013). Demographic data, primary site, grade and age at diagnosis were collected. Survival data was calculated from date of diagnosis to date of death in 166 cases. Results: $49 \%$ male and $51 \%$ female. $45 \%$ midguts $(51.8 \%$ G1, 15\% G2, 1.7\% G3), 23\% pancreatic (37.7\% G1, 25.5\% G2, $3.3 \% \mathrm{G} 3), 9 \%$ bronchial ( $48.7 \% \mathrm{G} 1,24.8 \% \mathrm{G} 2,4 \% \mathrm{G} 3), 7 \%$ appendiceal $(87.9 \%$ G1, $2 \%$ G2, no G3), $6.9 \%$ gastric, $5.4 \%$ hindgut and $4 \%$ other. Overall, $49.4 \%$ patients had G1, $17.6 \%$ had G2 and $2.5 \%$ had G3. $7.5 \%$ were diagnosed under the age of $30,38.1 \%$ between $30-55$ years, and $54.3 \%$ were over 55 . There was no evidence to support a relationship amongst high grade NETs and age at diagnosis, $r$ $(1301)=0.039, \mathrm{p}=-0.161 .166$ patients had died; 87 midgut patients (median survival 59 months), 47 pancreatic (42), 10 bronchial (36), and 11 hindguts (26). Higher grade tumours had a lower median survival. Conclusion: No evidence to support a relationship between age and grade of tumours. Grade 1 tumours were diagnosed more frequently than grade 2 and 3 combined. Midgut tumours have a higher median survival than pancreatic $>$ bronchial $>$ and $>$ hindgut tumours. Keywords: survival.

\section{Pathology, Grading, Staging}

\section{G1 \\ Interlaboratory Variability of MIB1 Staining in Well Differentiated Pancreatic Neuroendocrine Tumors}

Blank A., Wehweck L., Marinoni I., Boos L., Schmitt A., Perren A.

University of Bern, Institute of Pathology, Bern, Switzerland

Introduction: In neuroendocrine tumors (NET) grading and staging are the only routinely performed procedures to judge prognosis. Proliferation index using MIB1 staining has been introduced 
to assess grading. There are discussions on cut-off definitions, automated counting and inter-observer variability. $\mathbf{A i m}(\mathbf{s})$ : To assess Inter-lab differences, we performed MIB1 staining in 3 different University Hospital based pathology laboratories on a TMA of a well characterized patient cohort, containing pancreatic NET of 61 patients. Materials and Methods: To calculate the proliferation index, number of positive tumour nuclei was divided by the total number of tumour nuclei. Labelling index was compared to mitotic counts in whole tissue sections and to outcome. Linear regression analysis and ROC analysis were performed. Results: Inter-lab differences regarding intensity and number of positive cells in MIB1 staining were significant. ROC-analysis found 3 different 'optimal' cut offs for 3 different institutes $(2.1 \%, 0.79 \%$ and $0.4 \%)$. Every calculated cut off stratified the patient cohort to a significant extent for the underlying staining ( $\mathrm{p}=0.0009 ; 0.0010$ and 0.0020 ) but showed no or lesser significance when applied to the other stainings. Especially low proliferating tumours were affected by those differences. Conclusion: Significant inter-lab differences for MIB1 exist. Since the MIB1 proliferation index influences grading, local cut-offs or external standardization should be introduced to achieve reliability and reproducibility. Keywords: mib1, ki67, grading, net.

\section{G2 \\ Correlation between the 18F-FDG Uptake and Pathological Data in Well-Differentiated Digestive and Pulmonary Neuroendocrine Tumors (NET)}

Bucau M., Laurent-Bellue A., Cros J., Hentic O., Rebours V., Bedossa P., Ruszniewski P., Lebtahi R., Couvelard A.

AP-HP, HUPNVS Beaujon, Bichat \& Université Paris Diderot, Clichy, France

Introduction: The 18F-FDG uptake assessed by PET/CT is correlated to prognosis in most tumors. $\operatorname{Aim}(\mathbf{s})$ : The aim of our study was to compare the $18 \mathrm{~F}-\mathrm{FDG}$ uptake with tumor proliferation (Ki67) and expression of molecules related to cell metabolism [hypoxia (VHL), glucose transport (GLUT-1), cellular pH (CA9)] in well differentiated NET. Materials and Methods: This retrospective study included 22 patients. 18F-FDG uptake was assessed by the SUVmax value (Standardized Uptake Values). An immunohistochemical study was performed with Ki67, VHL, GLUT-1 and CA9 antibodies. Results: NET were of pancreatic (15), midgut (3), esophageal (1), rectal (1), pulmonary (1), undetermined origin (1). 7, 9 and 6 tumors were of grade 1, 2 and 3 respectively; the average/median Ki67 was $15.86 \% / 12 \%$ [1-60\%]. The mean/median SUVmax was 6.26/5.09 [1.47 to 18.70$]$. Two tumor with SUVmax $<2$ were considered as negative at PET scan. Grade 1, 2 and 3 tumors exhibited a median SUVmax 4.45 [1.62 to 6.6$] ; 5.79$ [1.46 to 18.7$]$ and 6.7 [3.05 to $9.61]$ respectively. The SUVmax correlated with high tumor size $(\mathrm{p}=$ $0.009)$, high Ki-67 ( $p=0.03)$ and low VHL expression $(\mathrm{p}=0.08)$. Conclusion: Our study highlights that well-differentiated NETs, even of grade 1, are frequently positive at PET scan. The accumulation of $18 \mathrm{~F}-\mathrm{FDG}$ increases with tumor size and cell proliferation and seems to be correlated with the expression of effectors of cellular metabolism. Keywords: neuroendocrine tumors, pet-scan, 18-fdg, ki67, vhl, metabolism.

\section{G3 \\ Significance of Ectopic Lymph Node-Like Structures in Neuroendocrine Tumors of the Small Bowel}

\author{
Cives M., Strosberg J., Simons S., Coppola D. \\ H. Lee Moffitt Cancer Center and Research Institute, \\ Tampa, Fla., USA
}

Introduction: The formation of extranodal tertiary lymphoid follicles and lymphoid cell aggregates has been described in nonmalignant conditions including autoimmune diseases and chronic inflammation as well as in cancer. Tumor-induced lymph node-like structures (LLS) participate in the host's antitumor immune response by driving intratumoral immune cell priming. $\operatorname{Aim}(\mathbf{s})$ : To evaluate the prognostic relevance and biologic significance of an adaptive immune reaction to small bowel NETs. Materials and Methods: Host immune response was evaluated by IHC in 60 well differentiated small bowel NETs. A miRNA microarray analysis was performed to identify a molecular signature of LLS presence. Results: In 13/60 samples the immune response was organized as ectopic LLS. When present, LLS were localized at the invasive edge of the tumors and contained germinal centers in different stages of maturation. CD21+ dendritic cells were present within the follicular germinal centers, while $\mathrm{CD} 3+\mathrm{T}$ cells were dispersed in the parafollicular cortex zone surrounding the CD20+ B cell area of the follicles. Time to progression of metastatic tumors was compared in an exploratory cohort of 5 patients with strong evidence of LLS vs. 5 patients with no evidence of LLS and was substantially longer in the former group (94 vs. 28 months; $p=0.15$ ). Updated survival and miRNA microarray analysis will be presented at the meeting. Conclusion: LLS may be useful for prognostic stratification and prediction of response to immune-based therapies. Keywords: immune microenvironment, lymph node-like structures.

\section{G4 \\ Expression and Clinical Significance of Merkel Cell Polyomavirus Large T Antigen in Merkel Cell Carcinoma}

\section{Delektorskaya V., Orlova K., Smirnova E., Demidov L. \\ Russian Cancer Research Centre, Moscow, Russia}

Introduction: Merkel cell carcinoma (MCC) is a rare and aggressive neuroendocrine skin cancer that is often associated with Merkel cell polyomavirus (MCPyV) infection. MCPyV expression and its relationship with clinical features and prognosis in MCC remain unclear. $\mathbf{A i m}(\mathbf{s})$ : The aim of the study was to investigate the frequency and pattern of the MCPyV expression in primary and 
metastatic tumors from patients with MCC and evaluate the role of $\mathrm{MCPyV}$ in prognosis of MCC. Materials and Methods: Merkel cell polyomavirus Large $\mathrm{T}$ antigen (MCPyV) expression was evaluated by immunohistochemistry with the monoclonal antibody CM2B4 in 32 pathologically confirmed MCC cases and correlated with clinicopathological parameters of patients. Results: MCPyV large $T$ antigen was expressed in various degrees in $24(75 \%)$ of 32 MCCs. Nuclear staining with heterogeneous intensity was identified in $19(59 \%)$, and $5(16 \%)$ specimens which showed positive labeling with CM2B4 in 50 to $100 \%$, and 10 to $50 \%$ of tumor cells, respectively. $8(25 \%)$ of 32 MCCs showed no nuclear positivity. There was no difference in MCPyV large $\mathrm{T}$ protein positivity between primary and metastatic lesions. MCPyV large $\mathrm{T}$ antigen expression showed only marginal association with longer progression-free survival in MCCs $(\log -r a n k p=0.050)$. Conclusion: MCPyV large T protein may represent a useful molecular marker with the potential to play an important role in the pathological diagnosis of MCC as well as improve its treatment. Keywords: merkel cell carcinoma, merkel cell polyomavirus, immunohistochemistry, prognosis.

\section{G5}

\section{Neuroendocrine Carcinomas of the Prostate}

\author{
Dema A. ${ }^{\text {, }}$ Taban S. ${ }^{\text {a }}$, Anderco D. ${ }^{\mathrm{b}}$, Bardan R. ${ }^{\mathrm{a}}$, \\ Daminescu L. ${ }^{\mathrm{b}}$, Cornea R. ${ }^{\mathrm{a}}$ \\ aUniversity of Medicine and Pharmacy, Timisoara, \\ Romania; 'b County Hospital, Timisoara, Romania
}

Introduction: Since focal neuroendocrine (NE) differentiation in prostate carcinomas represents a frequent phenomenon when NE markers are used for documentation, NE carcinomas are extremely rare form of highly aggressive prostate tumors. $\operatorname{Aim}(\mathbf{s})$ : This paper presents all cases of NE prostate carcinomas diagnosed in a Pathology Service from the western part of Romania. Materials and Methods: All the NE carcinomas of the prostate confirmed by immunohistochemistry in a period of 10 years were identified. Results: There were diagnosed 4 cases of NE prostate carcinomas in patients aged between 62 and 76 years: 1 case of NE small cell carcinoma (SCC) whose bladder or prostate origin was impossible to establish; 2 cases to whom, after previous hormonal treatment, was noticed transdifferentiation of primary conventional prostatic carcinoma in NE SCC and 1 case of mixed, conventional and NE SCC carcinoma. The NE differentiation was demonstrated by $\mathrm{CgA}$ positivity ( 3 cases) and/or Syn positivity (4 cases). In 3 of the 4 cases, the NE SCC component (from the prostate level or from a metastatic liver lesion) presented a positive reaction for TTF1. Conclusion: NE carcinomas represent an extremely rare subtype of prostate malignant tumors. Our findings draw attention to the possibility of NE transdifferentiation as a result of hormone deprivation therapy in patients with prostate cancer. TTF1-positivity in NE SCC of prostatic origin limits the value of this marker in determining the origin of a metastatic NE SCC. Keywords: neuroendocrine, carcinoma, prostate.

\section{G6 \\ Gastro-Entero-Pancreatic Mixed Adenoneuroendocrine Tumors. Report of Four Cases}

\author{
Koletsa T., Iliadis A., Tzioufa V., Karayannopoulou G. \\ Aristotle University of Thessaloniki, Thessaloniki, Greece
}

Introduction: Mixed adenoneuroendocrine carcinoma (MANECs) is an uncommon entity with ill-defined clinicopathologic characteristics. Aim(s): To present four cases of MANECs located on different sites of gastrointestinal tract. In addition, we aimed to investigate immunohistochemically the expression of epithelial-neuroendocrine markers, the proliferative index Ki67/MIB1 and MMR proteins. Materials and Methods: Four cases of tumors located on the gastro-esophageal junction, sigmoid, appendix and pancreas were included. Immunohistochemical study for epithelial, neuroendocrine markers, Ki67/MIB1 proliferative index, was performed. The microsattelite instabillity pathway (MSI) was immunohistochemically investigated, as well. Results: Histological examination of the tumors revealed mixed adenoneuroendocrine carcinomas. In all cases, immunohistochemical study helped to reach the precise diagnosis. MMR proteins were not expressed by neuroendocrine cells. Conclusion: MANECs can be found in any site of gastrointestinal tract and share common histological features. The neuroendocrine component of these presented MANECs did not express a variety of hormones. Microsatellite instabillity does not seem to play an important role in most MANECs. Keywords: mixed adenoneuroendocrine tumors, immunohistochemistry, mmr proteins, msi.

\section{G7 Clinico-Pathologic and Survival Analysis of 211 GastroEnteroPancreatic G3 Neuroendocrine Carcinomas (GEP-NECs)

\author{
Milione M. ', Spada F. ${ }^{\mathrm{b}}$, Maisonneuve P. ${ }^{\mathrm{b}}$, Pusceddu S. ${ }^{\mathrm{c}}$, \\ Spaggiari P. ${ }^{\mathrm{d}}$, Barberis M. ${ }^{\mathrm{b}}$, Albarello L. e , Vanoli A. ${ }^{\mathrm{f}}$, \\ Manzoni M.F.', Carnaghi C. ${ }^{\mathrm{d}}$, Fazio N. ${ }^{\mathrm{b}}$, Mazzaferro V. \\ Capella C.R. ${ }^{\mathrm{g}}$, La Rosa S. ${ }^{\mathrm{g}}$ \\ alRCCS Foundation National Cancer Institute, Milano, \\ Italy; 'bJEO, Milan, Milan, Italy; ' IRCCS Foundation National \\ Cancer Institute, Milan, Italy; ${ }^{\mathrm{I}} \mathrm{CH}$, Milan, Milan, Italy; \\ eSan Raffaele Hospital, Milan, Milan, Italy; fUniversity of \\ Pavia, Pavia, Italy; ${ }^{9}$ Ospedale di Circolo and University of \\ Insubria, Varese, Italy
}

Introduction: GEP-NECs show Ki67 $>20 \%$ and/or $>20$ mitoses x10 HPF. Mixed AdenoNeuroendocrine Carcinomas (MANECs) are mixed exocrine-neuroendocrine neoplasms. NECs are associated with poor prognosis but recent findings have suggested that there is a fraction of patients showing a better than expected survival rate. Aim(s): The present study correlates the prognosis of GEP-NECs/MANECs with clinicopathologic features. Materials and Methods: 211 NECs or MANECs were evaluated by 8 expert pathologists and stratified into the following subgroups: NECs vs. MANECs; 'low prolifer- 
ative' (LP) NECs (mitoses $<20$ and Ki67 between $20 \%$ and $55 \%$ ) vs. 'highly proliferative' (HP) NECs (mitoses $>20$ and Ki67>55\%) vs. MANECs. Prognosis was studied through Median Overall Survival (MOS). Results: 75 neoplasms were MANECs and 136 were NECs: 42 LP-NECs and 94 HP-NECs. The median follow-up time was 14 months. MOS was 12 months in NECs and 18 months in MANECs log-rank $\mathrm{P}=0.38$ ). However, when NECs were further stratified into LP and HP subgroups, a statistically different MOS was found. MOS was 29 months in LP-NECs, 6 months in HP-NECs and 18 months in MANECs $(p=0.002)$. At 6 months, the survival rate was $94 \%$ in the LP-NECs, $51 \%$ in the HP-NECs and $81 \%$ in MANECs. At 12 months it was $91 \%, 36 \%$ and $68 \%$, while at 36 months was $32 \%, 12 \%$ and $18 \%$, respectively. CD117 expression and angioinvasion were associated with poor prognosis. Conclusion: LP-NECs showed a better MOS than HP-NECs and MANECs. No differences in MOS were observed between MANECs and NECs. Keywords: nec, ki67, manec, prognosis.

\section{G8 \\ Histological Classification of Pancreatic Neuroendocrine Tumours: Optimising the Ki67 System}

\author{
Mills L. a, Ramage J. ${ }^{\mathrm{b}}$, Prachalias A. ${ }^{\mathrm{c}}$, Srinivasan P.

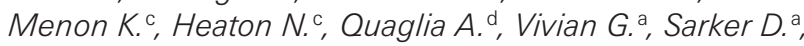 \\ Ross P. ${ }^{a}$ Basuroy R. ${ }^{\text {a }}$ Srirajaskanthan R. ${ }^{\mathrm{e}}$ \\ aENETS Centre of Excellence, Institute of Liver Studies, \\ King's College Hospital, London, UK; ' Department of \\ Gastroenterology, Hampshire Hospitals NHS Trust, \\ Basingstoke, UK; cHepatopancreatobiliary Surgery, \\ Institute of Liver Studies, King's College Hospital, \\ London, UK; d Department of Histopathology, Institute \\ of Liver Studies, King's College Hospital, London, UK; \\ eDepartment of Gastroenterology, University Hospital \\ Lewisham, London, UK
}

Introduction: Pancreatic neuroendocrine tumours are graded using the Ki67 antigen in accordance with the WHO 2010 classification. Despite the system's widespread use, a wide variety of tumour behaviours have been described leading to various proposed revisions. Aim(s): To investigate: a) the use of 5\% rather than $2 \%$ as the boundary between Grades 1 and 2, b) the use of 55\% rather than $20 \%$ as the boundary between Grades 2 and 3, c) the subdivision of Grade 3 using poorly vs. well-differentiated appearances. Materials and Methods: Of 223 patients who visited our institution between 2004 and 2013, Ki67 values were known for 168 . Where data was available for the pancreas and liver metastases, the highest value was used. The significance of each system was investigated using Kaplan-Meier survival curves and specific Ki67 values searched for using ROC. Results: a) G1 vs. G2 prognostic significance was not improved using $5 \%$ instead of $2 \%$. G3 vs. G1/G2 remained significant ( $p<$ $0.001)$. b) G2 vs. G3 remained significant using 55\% ( $<<0.001)$. G1 vs. G2 significance improved compared to $20 \%$ ( $\mathrm{p}=0.019$ vs. 0.193 ). ROC analysis showed that $\mathrm{Ki} 67=55 \%$ had $100 \%$ specificity, but that Ki67 values between $10 \%$ and $20 \%$ offer the best specificity/ sensitivity balance. c) Defining G3 using poor-differentiation rather than $>20 \%$ improved G1 vs. G2 ( $p=0.133$ vs. 0.195$)$. Conclusion: 1) Prognostic relevance of Grades 2 and 3 may be improved by using either $55 \%$ or poor-differentiation as criteria. 2) There is no evidence to suggest that $5 \%$ offers increased prognostic relevance for Grade 1. Keywords: g3.

\section{Biomarkers}

\section{H1 \\ Neuroendocrine Gene Transcript Analysis of Blood Identifies Stable or Progressive NET Disease Treated with Somatostatin Analogs}

\author{
Alaimo D., Callahan S., Lepensky C., Jonzzon S., \\ Teixeira N., Bodei L., Drozdov I., Kidd M., Modlin I. \\ Wren Laboratories, Branford, USA
}

Introduction: An issue in NET management is defining stable or progressive disease and effective treatment. An alternative to RECIST criteria is a sensitive, reproducible blood biomarker test. Somatostatin analogs (SSA) are a chief therapy but efficacy is subjective and based on symptom control and imaging. Aim(s): Investigate the utility of a PCR-based 51 gene signature (NETest) and CgA as biomarkers to define SSA efficacy. Materials and Methods: GEP-NETs $(\mathrm{n}=$ 35) M:F 12:23; median age 58, small intestine $\mathrm{n}=29$, pancreas $\mathrm{n}=$ 3 , rectum $n=3$ were evaluated. qRT-PCR measured transcript levles and ELISA (DAKO) for CgA. Clinical status and imaging was assessed. NETest defines disease activity risk scaled $0-100 \%$; low and high activity cutoff at $47 \%$. Results: SSA therapy: Sandostatin, $\mathrm{n}=33$, octreotide, $\mathrm{n}=2$, pasireotide, $\mathrm{n}=1$, dose range $20-60 \mathrm{mg}$. In stable disease group (SD) $\mathrm{n}=25,71 \%$ NETest was significantly lower $(32 \pm 3.7 \% ; \mathrm{n}=25)$ than progressive disease group (PD: $82 \pm$ $4.3 \%, \mathrm{n}=10 ; \mathrm{p}<0.0001)$. The accuracy for determining SD from PD was $92 \%$, with sensitivity $100 \%$ and specificity $88 \%$. CgA levels were insignificant $(30.6 \pm 7.8 \mathrm{U} / \mathrm{L}$ vs. $93.5 \pm 73 \mathrm{U} / \mathrm{L}, \mathrm{p}=0.4)$; accuracy for identifying SD from PD was $60 \%$; sensitivity and specificity $60 \%$. The NETest significantly outperformed CgA with AUCs $0.97 \pm$ 0.025 , and $0.60 \pm 0.06$ respectively (difference 0.37, Z-statistic 3.36, $\mathrm{p}=0.0008)$. Conclusion: NET transcript analysis correctly identified SD and PD activity with SSA treatment. Blood-based NETest will enable early detection of progression and define SSA efficacy. Keywords: biomarker, ssa. 
$\mathrm{H} 2$

\section{An Isolated Abnormal Chromogranin B Is Associated with Pancreato-Duodenal NETs and the Presence of Liver Metastases}

\author{
Basuroy R., Sherwood R., Srirajaskanthan R., Ramage J. \\ KHP ENETS Center of Excellence, King's College Hospital, \\ London, UK
}

Introduction: Chromogranin $\mathrm{B}(\mathrm{CgB})$ is a soluble secretory protein like chromogranin $\mathrm{A}(\mathrm{CgA})$ that has been reported to be elevated in patients with neuroendocrine tumours (NETs) but is not routinely assessed. Aim(s): This study explores whether an abnormal CgB paired with a normal $\mathrm{CgA}$ provides any useful clinical information in NET patients. Materials and Methods: A prospective NET database (01/2007-11/2014) was interrogated for paired radioimmunoassay $\mathrm{CgA}$ (two assays; normal $<6 \mathrm{nmol} / \mathrm{L} \& 60 \mathrm{pmol} / \mathrm{L}$ ) and $\mathrm{CgB}$ (two assays, normal $<3 \mathrm{nmol} / \mathrm{L},<150 \mathrm{pmol} / \mathrm{L}$ ) results. Results: 1161 paired CgA \& $\mathrm{CgB}$ results (479 patients) were retrieved. 51 patients had an isolated abnormal paired $\mathrm{CgB}$ (79 results; mean $\mathrm{CgB}$ result $=$ $2.68 \mathrm{xULN}) ; 2$ patients were excluded as coding error. Primary sites were 24 pancreas, 9 small bowel, 4 rectal, 3 duodenal, 3 unknown, 2 duodenal-jejunal, 2 pre-sacral, 1 caecal and 1 lung. In 8/9 small bowel NETs, the $\mathrm{CgB}$ was raised in isolation on only one occasion on longitudinal testing. The tumour grades were $24 \mathrm{G} 1,18 \mathrm{G} 2,4$ G3 and 3 unknown. Liver metastases were present in 38/49 patients. Conclusion: An isolated abnormal paired $\mathrm{CgB}$ was observed in $11 \%$ of NET patients. In $59 \%$ of patients it was associated with a pancreatic or duodenal primary NET. In $78 \%$ liver metastases were present. Further assessment is required to establish the role of $\mathrm{CgB}$ when paired with $\mathrm{CgA}$ as a biomarker of clinical utility in the diagnosis, prognosis and therapeutic monitoring of NETs. Keywords: chromogranin a, chromogranin b, pancreas, duodenal, assay, liver metastases.
H3

Correlation of Serum Plasma Chromogranin
A with Survival and Disease Features in
Patients with Gastroenteropancreatic
Neuroendocrine Tumors. First Results in a
Large Database in Argentina. ARGENTUM
GROUP

Belli S. ${ }^{\mathrm{a}}, \mathrm{O}^{\prime}$ Connor J.M. ${ }^{\mathrm{b}}$, Oneto A. ${ }^{\mathrm{c}}$, Pesce V. ${ }^{\mathrm{d}}$, Bestani C. ${ }^{\mathrm{e}}$, Marmissolle F.', Domenichini E. 9, Roca E. ${ }^{\mathrm{e}}$

aAlexander Fleming Institute, Carlos G. Durand Hospital,

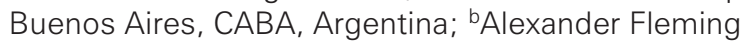
Institute, and Gastroenterology Hospital, B. Udaondo, Buenos Aires, CABA, Argentina; ' Carlos G. Durand Hospital, Buenos Aires, CABA, Argentina; dMarie Curie Hospital, Gastroenterology Hospital, B. Udaondo, Buenos Aires, CABA, Argentina; ' Gastroenterology Hospital, B. Udaondo, Buenos Aires, CABA, Argentina; 'SITE, La Plata, La Plata, Buenos Aires, Argentina; ${ }^{9}$ Alexander Fleming Institute, Buenos Aires, CABA, Argentina

Introduction: The plasma chromogranin $\mathrm{A}(\mathrm{CgA})$ is the best general biomarker in gastroenteropancreatic neuroendocrine tumors (GEP-NETs). CrA levels are elevated in a variety of GEP-NETs and are used to aid in diagnosis, prognosis, and monitoring of treatment effects in this population. Aim(s): To correlate the baseline value and change CrA levels with patient survival and tumor response in GEP-NETs. Materials and Methods: Serial CgA determinations were performed in 280 patients in different Centers in our Country. $\mathrm{CgA}$ level was analysed for correlation with the patient's clinical outcome, looking for survival as a primary end-point. CgA was measured at baseline and at regular intervals after diagnosis. Results: There was female preponderance (54\%). Median age of patients was 57 years, (range 44 to 64 years). Primary site was small bowel $31 \%$, pancreas $26 \%$ and unknown $17 \%$ among the most frequent primary location. Twenty nine percent of the patients had functional tumor. Median CgA level was $15 \mathrm{nmol} / \mathrm{l}$. (range: 1 to $8000 \mathrm{nmol} / \mathrm{l}$ ). Baseline plasma CgA above $10 \mathrm{nmol} / 1$ (normal value up to 10) was an independent prognostic factor HR: $2.70,95 \%$ CI $1.09-6.68$, p: 0.031 . The changes of the CgA level $(\triangle \mathrm{CgA})$ above $50 \mathrm{nmol} / \mathrm{l}$ shows correlation in terms of response rate HR: $0.44,95 \%$ CI $0.24-0.83$, $\mathrm{p}$ value: 0.011 ). Conclusion: In our GEP-NETs patients the baseline plasma $\mathrm{CgA}$ level showed a significant correlation with survival and $\triangle \mathrm{CgA}$ predicted treatment response in our study population. Keywords: neuroendocrine tumor, chromogranin a, prognostic factor. 


\section{H4 \\ Impact of VEGF and VEGFR Polymorphisms on Neuroendocrine Tumors of the Gastro-Entero-Pancreatic System (GEP-NET) Outcome}

\author{
Berardi R. ${ }^{a}$, Pagliaretta S. ${ }^{\text {a }, ~ R i n a l d i ~ S . ~}{ }^{\text {, }}$, Morgese F. \\ Partelli S. ${ }^{\mathrm{b}}$, Caramanti M. ${ }^{\mathrm{a}}$, Onofri A. ${ }^{\mathrm{a}}$, Polenta V. \\ Pasquini S. ${ }^{c}$, Falconi M. ${ }^{\text {, }}$ Cascinu S. ${ }^{a}$ \\ aUniversità Politecnica delle Marche-Clinica di Oncologia \\ Medica, Ancona, Italy; bUniversità Politecnica delle \\ Marche-Chirurgia del Pancreas, Ancona, Italy; 'Università \\ di Bologna-Facoltà di Scienze Statistiche, Ancona, Italy
}

Introduction: Improving our understanding of the molecular biology of GEP-NETs represents a key challenge in the treatment of these rare tumors. Aim(s): We aimed to analyze genotypes of VEGF-A, VEGFR2 and Flt4 in GEP-NETs and their potential correlation with the risk of these tumors and/or with the outcome. Materials and Methods: The genomic DNA of 28 consecutive patients with GEP-NETs treated at our Institution was extracted from paraffin-embedded tissue. We selected polymorphisms in the following genes: VEGF-A (rs2010963G>C, rs699947A>C), VEGFR-2 (rs2305948C $>$ T, rs $1870377 \mathrm{~T}>\mathrm{A})$ and VEGFR-3 (rs307826T $>\mathrm{C}$, rs307821C $>$ A). Gene polymorphisms were determined by RealTime PCR using TaqMan assays. Results: Median age was 58 (range 33-78), M/F ratio was 1/1;21 patients had NETs of the pancreas. The allele frequency of VEGFR2 rs2305948C and of VEGFA rs $2010963 \mathrm{G}$ showed a trend of lower frequency than in general population ( $82 \%$ vs. $92 \%$ and $60.3 \%$ vs. $68.8 \%$, respectively). The following factors significantly correlated with a poorer overall survival: VEGFA rs699947C, VEGFA rs2010963A, VEGFR-2 rs1870377T/A, VEGFR-2 rs2305948C/T, VEGFR-3 rs307821C and VEGFR-3 rs307826T. Conclusion: Our results suggest, for the first time, that inherited abnormalities in VEGF pathway influence the risk and aggressiveness of GEP-NETs. Within March 2015 final data about the enrolled 40 patients will be also available. These results should be validated prospectively in order to optimize the diagnosis and treatment of these patients. Keywords: angiogenesis, prognosis, neuroendocrine tumors.
H5

\section{Blood Gene Transcript Analysis Predicts 68Ga-SSA-PET/CT Imaging in Neuroendocrine Tumors and Defines Disease Status}

Bodei L. ${ }^{a}$, Kidd M. ${ }^{\mathrm{b}}$, Modlin I. ${ }^{\mathrm{b}}$, Drozdov I. ${ }^{\mathrm{b}}$, Prasad V. ${ }^{\mathrm{c}}$

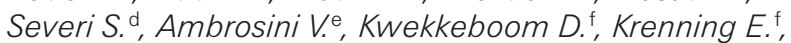
Baum R. ${ }^{g}$, Paganelli G. $^{\mathrm{d}}$

aDivision of Nuclear Medicine, European Institute of Oncology, Milan, Italy; 'Wren Laboratories, Branford, Conn., USA; 'Department of Nuclear Medicine, Charité University Hospital, Berlin, Germany; ${ }^{d}$ Nuclear Medicine and Radiometabolic Units, Istituto Scientifico Romagnolo per lo Studio e la Cura dei Tumori (IRST) IRCCS, Meldola, Italy; 'Nuclear Medicine Department, S.Orsola-Malpighi University Hospital, Bologna, Italy; ${ }^{f}$ Nuclear Medicine Department, Erasmus Medical Center, Rotterdam, The Netherlands; ${ }^{9}$ Theranostics Center for Molecular Radiotherapy and Imaging, Zentralklinik Bad Berka, Bad Berka, Germany

Introduction: Limitations of biomarkers and imaging are a concern in the identification of NET status and therapy response. $\operatorname{Aim}(\mathbf{s})$ : To identify if circulating molecular markers and imaging provided added value, we assessed the utility of $68 \mathrm{Ga}$-SSA-PET, $\mathrm{Ki}-67, \mathrm{CgA}$, and circulating NET transcripts (NETest). Materials and Methods: In two independent datasets (1, pre-PRRT, $n=27 ; 2$, follow up, $\mathrm{n}=22$ ) we evaluated SUVmax, Ki-67, CgA and NETest (regression analyses, generalized linear modeling, ROC analyses). Results: 68Ga-SSA-PET was positive in all. In 47/49 (96\%) NETest was positive. $\mathrm{CgA}$ was elevated $26 / 49(54 \%)\left(\chi^{2}=20.1, \mathrm{p}<2.5 \times 10\right.$ $6)$. NETest was predictive of imaging ( $>95 \%$ concordance). Most lesions $(\sim 80 \%)$ were Grade I/II. Ki-67 and $\mathrm{CgA}$ were unrelated and had no relationship to imaging parameters. Blood transcript levels significantly correlated with SUVmax with highest correlation for the genes MORF4L2 and SSTR1, 3, $5\left(\mathrm{R}^{2}=0.31\right.$ and 0.15 , $\mathrm{RMSE}=9.4$ and 9.5, respectively). Elevated quotients of MORF4L2 and SUVmax identified progressive disease $\left[(\mathrm{p}<0.03)\right.$, ROC-derived AUCs $\left(\mathrm{R}^{2}=\right.$ $0.7, p<0.05)]$. Conclusion: SUVmax measured in two centers was mathematically comparable and predicted by circulating transcript levels. NET disease stability/progress determined by imaging can be quantified by circulating transcripts. Such analyses may facilitate less frequent SSR PET/CT usage. Fusion of circulating molecular signatures and imaging parameters will provide added clinical utility by delineating tumor functional and genomic status. Keywords: 68gassa-pet, netest, transcripts, pcr. 
H6

\section{Measurement of Blood Gene Transcripts Defines PRRT Therapeutic Efficacy}

Bodei L. a, Paganelli G. b. Kidd M. ${ }^{c}$, Severi S. ${ }^{\text {, }}$, Lepensky C. ${ }^{c}$, Alaimo D.c, Callahan S. c, Drozdov I.', Kwekkeboom D. , Krenning E. d, Baum R. e, Modlin I. ${ }^{\dagger}$

aDivision of Nuclear Medicine, European Institute of Oncology; LuGenlum Consortium, Milan, Bad Berka,

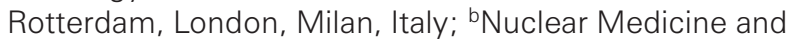
Radiometabolic Units, Istituto Scientifico Romagnolo per lo Studio e la Cura dei Tumori (IRST) IRCCS, Meldola, Italy; 'Wren Laboratories, Branford, Conn., USA; 'Nuclear Medicine Department, Erasmus Medical Center;

LuGenlum Consortium, Milan, Bad Berka, Rotterdam, London, Rotterdam, The Netherlands; 'Theranostics Center for Molecular Radiotherapy and Imaging, Zentralklinik Bad Berka; LuGenlum Consortium, Milan, Bad Berka, Rotterdam, London, Bad Berka, Germany; fWren Laboratories; LuGenlum Consortium, Milan, Bad Berka, Rotterdam, London, Branford, Conn., USA

Introduction: PRRT is an effective therapy for NETs since the majority over-express SSRs. Typically, well or moderately differentiated NETs (G1/G2) are treated. Imaging and $\mathrm{CgA}$ are used to evaluate response but both RECIST criteria and CgA have limitations. Aim(s): Assess efficacy of a multi analyte algorithmic (MAAA) blood gene transcript test and $\mathrm{CgA}$ in defining PRRT efficacy. Materials and Methods: NETs $(\mathrm{n}=32)$, M: F 22:10, median age 67, bronchial: $\mathrm{n}=$ 8, GEP-NET: $\mathrm{n}=22$, CUP: $\mathrm{n}=2$ were evaluated. $19 / 32$ were G1/G2, 19/29 were FDG+ve and 24/32 had progressive disease (PD). Status was assessed clinically and with imaging (RECIST). Cumulative Lu-PRRT activity was 4.6-27.8 GBq, median 18.5. Transcript analysis was by qRT-PCR and ELISA for CgA (EuroDiagnostica). The MAAA NETest mathematically delineates disease activity risk: scale 0-100\%; minimal $<20 \%$, low: $20-47 \%$, high $>47 \%$. Results: All patients had a positive NETest prior to PRRT. Average score was $34 \pm$ $5 \%$. CgA was elevated in 18/32 $(976 \pm 313 \mathrm{ng} / \mathrm{ml})$. NETest scores increased up to PRRT Cycle II $(47 \pm 5 \% ; \mathrm{p}<0.05)$. By Cycle IV, they significantly decreased $(-25 \%, \mathrm{p}<0.02)$. Individual scores correlated with outcome. Stable Disease/Partial Response: $(n=27: 22 \pm$ $2 \%)$ vs. Progressive Disease $(n=5: 71 \pm 4 \%, p<0.0001)$. CgA levels were overall unchanged $(p=0.43)$, remaining elevated. Conclusion: Blood gene transcript levels correlated with and defined PRRT efficacy while $\mathrm{CgA}$ was non-informative. Keywords: gene transcripts, netest, neuroendocrine, prrt.
H7

\section{The Association between Gastrin and Glucose Serum Concentration}

Boutzios G. ${ }^{\text {, }}$ Alexandraki K. ${ }^{a}$, Moschouris P.a , Chatzellis E. ${ }^{\text {, }}$ Evaggelatou A. ${ }^{\mathrm{b}}$, Velidaki $A .{ }^{\mathrm{b}}$, Kitsou E. ${ }^{\mathrm{b}}$, Kaltsas $\mathrm{G} .{ }^{\mathrm{a}}$

${ }^{a}$ Endocrine Oncology Unit, Section of Endocrinology,

Department of Pathophysiology, National and

Kapodestrian University of Athens Medical School,

Athens, Greece; 'bepartment of Nuclear Medicine, Laiko

University Hospital, Athens, Greece

Introduction: Gastrin is an early incretin candidate, since it is released by oral glucose and potentiates the glucose-induced insulin secretion. It has been shown that only in hypoglycemic or hyperglycemic conditions gastrin release is influenced by changes in blood glucose and insulin concentrations. Aim(s): To evaluate whether there is a relation between fasting serum glucose and serum gastrin concentrations. Materials and Methods: Gastrin and glucose levels and $\mathrm{HbA} 1 \mathrm{c}$ were measured in 134 blood samples. We studied gastrin serum concentrations dividing the samples in 3 subgroups with glucose concentrations $<100 \mathrm{mg} / \mathrm{dl}, 101-200 \mathrm{mg} / \mathrm{dl},>201 \mathrm{mg} / \mathrm{dl}$, and in 3 subgroups with $\mathrm{HbA} 1 \mathrm{c}<5.7 \%, 5.7-6.4 \%,>6.5 \%$. Results: No correlation was found between gastrin and glucose in the total population of samples studied. No difference was found in gastric concentration in the subgroups of normal, moderately increased, increased glucose concentrations $(454.7 \pm 333.5,422.4 \pm 313.2,210.6 \pm 62.5$, respectively), or in the subgroups of normal, prediabetic, diabetic range of HbAlc $(534 \pm 276.3,488.6 \pm 339,440.1 \pm 309.8$, respectively). Conclusion: Despite the previous studies which have shown some correlation between glucose and gastrin levels, no such association was seen in the specific study. Keywords: gastrin levels, glucose levels, hbalc.

\section{H8}

\section{Changes in Neuroendocrine Tumor Microenvironment with WHO Tumor Grading}

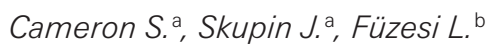

${ }^{a}$ Gastroenterology and Endocrinology, University Medicine Göttingen, Göttingen, Germany; 'bathology, University Medicine Göttingen, Göttingen, Germany

Introduction: The microenvironment of neuroendocrine neoplasias (NEN) in view of tumor grading (WHO classification of 2000 und 2010) has not yet been validated. Both classifications distinguish between well and poorly differentiated neuroendocrine tumors, including proliferation rate, mitotic index and histomorphology. The WHO classification of 2000 further evaluates tumor location, size, angioinvasion, invasion of the muscle layer, functionally active tumors and metastases. Aim(s): We analysed tumor and stromal markers in view of these classifications. Materials and Methods: 55 different primary tumors were analysed: 32 small intestinal, 13 colorectal and 10 pancreatic NEN. Immunohistochemistry was performed on paraffin sections. As tumormarker chromogranin, CD56 and S100 were used. 
Stromal markers were $\alpha$-SMA and Desmin. Markers for endothelial cells were CD34 and vWF. CD68 and KiM1P were used as markers for macrophages and fibrohistiocytes. Lymphocytes were stained with $\mathrm{CD} 3+$ for T-cells and CD20+ for B-cells. Analysis of cellcount and staining-intensity was computer-based and visually controlled. Results: Immunohistochemistry showed a decreased tumor stroma and reduced expression of tumor-differentiation markers with increased malignity. Fibrohistiocytic cells were the most common immune cell population, followed by CD3+ cells. The number of immune cells increased with WHO-grading. This difference was more explicit, when the WHO-classification of 2000 was applied. Conclusion: The microenvironment plays a role for NEN progression. Keywords: nen, stroma.

\section{H9 \\ The Neuron-Specific Enolase Assessment in Patients with Neuroendocrine Tumors with or without Carcinoid Syndrome}

Carsote M. a, Capatina C. ${ }^{\text {a }}$ Terzea D. ${ }^{b}$, Petris R. ${ }^{c}$, Goldstein A. ', Hortopan D. ', Dumitrascu A. ', Trifanescu R. ${ }^{\text {, }}$ Paun D. ${ }^{\text {a }}$ Poiana $C$. $^{\text {a }}$

${ }^{a}$ Carol Davila University of Medicine and Pharmacy, C.I. Parhon National Institute of Endocrinology, Bucharest, Romania; ${ }^{\mathrm{b}} \mathrm{Carol}$ Davila University of Medicine and Pharmacy, C.I. Parhon National Institute of Endocrinology, Monza Hospital, Bucharest, Romania; 'C C.I.Parhon National Institute of Endocrinology, Bucharest, Romania

Introduction: NeuroEndocrine Tumors (NETs) comprises a wide area of clinical presentations and neuroendocrine markers. $\operatorname{Aim}(\mathbf{s})$ : We focused on Neuron-Specific Enolase (NSE) assay in NETs. Materials and Methods: This is an observational study in patients with NETs, with at least 1 endocrine evaluation at C.I.Parhon National Institute of Endocrinology, Bucharest, between 2008-2014. Clinical evaluation (including the confirmation of carcinoid syndrome), neuroendocrine markers were assessed: chromogranin A (normal $<100 \mathrm{ng} / \mathrm{mL}$ ), serotonin (normal $<240 \mathrm{ng} / \mathrm{mL}$ ), and serum NSE (normal $<12 \mathrm{ng} / \mathrm{mL}$ ). Results: Out of 47 patients confirmed with NETs we were able to perform the NSE in 28. The female/ male ratio: 13/15. The age ranges: between 22 and 87 years at first presentation (median of 53). 18 patients had normal NSE. $50 \%$ of them had typical carcinoid syndrome. The grading was: G1/G2/G3 of $11 / 3 / 4$ patients in each group. 8 of 18 patients with normal NSE had high serum serotonin, and a number of 8 patients had abnormal chromogranin A. 10 patients with NSE above normal (median of 20.5 $\mathrm{ng} / \mathrm{mL}$; median of 54.5 years); only 1 patient $(10 \%)$ had normal levels of chromogranin A and serotonin. The number of patients was: G1-4, G2-4, G3-2. no NSE-chromogranin A or NSE-. Conclusion: Neuron-Specific Enolase might be a prognostic and follow-up marker useful in specific types of neuroendocrine tumors, especially those wthose with baseline abnormal level. Whether NSE is a general screening tool it is difficult to established. Keywords: neuron-specific enolase, neuroendocrine tumors.

\section{H10 \\ Chromogranin A (CgA) in \\ Pheochromocytoma and in Multiple \\ Endocrine Neoplasia Type 1}

Glinicki P. ${ }^{a}$ Jeske W.a, Bednarek-Papierska L. b,

Rosłonowska E. ', Zgliczyński W. ${ }^{\text {a }}$

aThe Centre of Postgraduate Medical Education, Department of Endocrinology, Warsaw, Poland; ' ${ }^{\mathrm{T}}$ The

Centre of Postgraduate Medical Education, Department of Endocrinology, Warsaw, Poland; 'Department of Internal

Diseases, Metabolism and Endocrinology, The Bielanski

Hospital, Warsaw, Poland

Introduction: Chromogranin $\mathrm{A}(\mathrm{CgA})$ as a main, nonspecific marker of neuroendocrine tumors (NET) has been applied for diagnostic purposes and for monitoring the treatment of NET. Pheochromocytoma and MEN-1 syndrome represent rare neuroendocrine tumours. $\mathbf{A i m}(\mathbf{s})$ : The aim of the study was to evaluate the usefulness of CgA measurements in patients with pheochromocytoma and multiple endocrine neoplasia type 1 . Materials and Methods: 20 patients with pheochromocytoma and 12 patients with MEN-1 syndrome. Chromogranin A was measured by immunoradiometric method (CIS bio, France). Results: In 17 of 20 of patients with pheochromocytoma plasma $\mathrm{CgA}$ levels were markedly elevated and ranged 180-1971 ng/ml (median $425 \mathrm{ng} / \mathrm{ml}$ ), but in 3 subclinical cases CgA levels were not elevated (79-131 ng/ml). The cut-off value established for plasma samples was $152 \mathrm{ng} / \mathrm{ml}$ ( $\mathrm{p}<0.0001$, AUC 0.9596 ), received $80 \%$ sensitivity and $93 \%$ specificity. In patients with MEN-1 $(\mathrm{n}=12)$, the median CgA concentration in serum was $158 \mathrm{ng} / \mathrm{ml}(34-1800 \mathrm{ng} / \mathrm{ml})$. The median CgA concentration in NET tumors forming part of the MEN 1 (including pancreatic gastrinoma and carcinoid) was $974 \mathrm{ng} / \mathrm{ml}(216-1800 \mathrm{ng} / \mathrm{ml})$, which resulted with $58 \%$ sensitivity and $100 \%$ specificity for the cut-off point of $100 \mathrm{ng} /$ $\mathrm{ml}(\mathrm{p}=0.0012$, AUC 0.8222). Conclusion: CgA might be a useful additional tumour marker of pheochromocytoma and multiple endocrine neoplasia type 1. Keywords: chromogranin a, cga, pheochromocytoma, multiple endocrine neoplasia, neuroendocrine tumors.

\section{H11 \\ Tumor Infiltrating Lymphocytes and PD-L1 Expression Differ in Low and High Grade Neuroendocrine Tumors}

Grabowski P. ${ }^{\mathrm{a}}$ Joehrens K. ${ }^{\mathrm{b}}$, Arsenic R. ${ }^{\mathrm{b}}$, Kaemmerer D. ${ }^{\mathrm{c}}$, Hummel M. ${ }^{\mathrm{b}}$, Scheibenbogen C. ${ }^{\mathrm{a}}$, Busse A. ${ }^{\mathrm{d}}$

${ }^{a}$ Charité-Institute of Immunology, Berlin, Germany; ${ }^{b}$ Charité-Institute of Pathology, Berlin, Germany;

cZentralklinik Bad Berka, Department of Visceral Surgery, Bad Berka, Germany; dCharité-Hematology, Oncology and Tumorimmunology, Berlin, Germany

Introduction: Although much progress has been made in the past 2-3 years in terms of understanding signaling pathways in gastroenteropancreatic neuroendocrine tumors (GEP-NENs), approved 
treatment options are still limited. Cancer Immunotherapy has been announced as the breakthrough of the year in 2013 and might become an integral part of the clinical management strategy for solid tumors including GEP-NENs. Therefore, to develop immunotherapeutic strategies against NENs we need to know which immune escape mechanisms are relevant in high and low grade NEC/NENs. $\operatorname{Aim}(\mathbf{s})$ : We aim to characterize the tumor microenvironment (TME) of NEN/NECs on the cellular level in order to define relevant immunophenotypes. Materials and Methods: TMAs of 70 poorly differentiated neuroendocrine carcinomas (PDECs) of all localizations and 20 well differentiated ilial neuroendocrine carcinomas (WDNET) of two different institutions (Charite-Universitätsmedizin Berlin and Zentralklinik Bad Berka) were stained for CD3 expression; 50 PDECs and 20 WDNETs were stained for PD-L1 expression. Results: More CD3 positive lymphocytes were seen in PDECs than in WDNET. In addition, PD-L1 expression was more frequently observed on tumor cells in 50 PDECs (of all locations) than in 20 WDNETs of ileal localization. More data and correlation with clinical data will be shown on the meeting. Conclusion: Our preliminary data indicate that PD1/PD-L1 may be promising targets for immunotherapy especially in poorly differentiated NECs. Keywords: immunophenotype, pd-11, poorly differentiated necs, immunotherapy.

\section{H12 \\ Circulating Gene Transcript Analysis of Pancreatic NETs}

Kidd M. a, Modlin I. ${ }^{a}$, Drozdov I. ${ }^{\text {, }}$ Alaimo D. ${ }^{\text {a }, ~ C a l l a h a n ~ S . ~}{ }^{\text {, }}$ Aslanian H. ${ }^{\text {b }}$ Lepensky C. ${ }^{\text {, }}$ Teixeira N. ${ }^{\text {a }}$, Bodei L. ${ }^{\text {a }}$

aWren Laboratories, Branford, USA; bYale University

School of Medicine, New Haven, USA

Introduction: PNETs are aggressive with poor survival. Therapeutic agents are available but efficacy determination is limited by imaging insensitivity and the lack of effective blood biomarkers. Aim(s): Examine the utility of a blood transcript signature in comparison to CgA measurement. Materials and Methods: We assessed 2 separate prospectively collected datasets. Set 1: Graded PNETs ( $\mathrm{n}=65 ; 25$ resections). Set 2 : unknown pancreas disease $(\mathrm{n}=61$ : pancreatitis $\mathrm{n}=4$, cyst $\mathrm{n}=31$, NET $\mathrm{n}=4$, adenoCa $\mathrm{n}=$ 22). Set 1 and 2: Gene expression (qPCR) and CgA (DAKO) were compared $(\chi 2$, non-parametric). Metrics were calculated for the entire cohort $(n=126)$. Status was assessed clinically + RECIST. NETest index defined disease activity scaled $0-100 \%$; minimal $<20 \%$, low $20-47 \%$, high $>47 \%$. Results: Set 1 : PCR positivity was $61 / 65(94 \%)$ and $\mathrm{CgA}$ positivity was $34 \%\left(\mathrm{Chi}^{2}=50.7, \mathrm{p}<\right.$ 10-13). PCR index in G1 or G2 tumors was similar (79.4 $\pm 6 \%$ vs. $72.5 \pm 6 \%$ ). Index was significantly higher in progressive (PD) compared to stable disease (SD) $(65 \pm 5 \%$ vs. $32 \pm 8 \%, \mathrm{p}<0.005)$. Palliative surgery reduced the score $(-29.4 \%, \mathrm{p}<0.02)$. Set 2 : PCR was positive in $7 / 61 ; 4$ PNETs, 2 IPNMs, 1 pancreatic Ca. False +ve rate $4.9 \%$. Metric analysis NETest $/ \mathrm{CgA}: 94 \% / 33 \%$, specificity $96 \% / 61 \%$, PPV $96 \% / 51 \%$ and NPV 93\%/43\%. Conclusion: The NETest is specific for PNETs. Levels correlate with cytoreduction and differentiate SD from PD. The test significantly outper- forms CgA. Circulating gene transcripts accurately diagnose PNETs and disease progress facilitating assessment of treatment efficacy. Keywords: pnet, netest, pcr.

H13

The Clinical Utility of Blood Derived Gene Cluster Analysis of Neuroendocrine Tumors Using Neoplasia Hallmark Criteria

Kidd M., Drozdov I., Modlin I.

Wren Laboratories, Branford, Conn., USA

Introduction: We developed a multianalyte blood (51 transcript) assay (MAAA) for GEP-NETs with a sensitivity (98\%) and specificity (97\%). Aim(s): Examine tissue expression levels to define, by cluster analysis, the biological 'hallmarks' of NET neoplasia and clinical utility. Materials and Methods: Group 1: Expression in 2 NET transcriptome databases $(n=15)$ assessed by GeneProfiler and by PCR (tissue with matched blood: $\mathrm{n}=24)$. Group $2(\mathrm{n}=$ 138): stable disease (SD): $\mathrm{n}=94$; progressive disease (PD): $\mathrm{n}=44$. Validation Groups: surgical resection $(n=27)$ and SSA treatment $(\mathrm{n}=35)$. Regulatory network analysis, PCA, and ROC were used to delineate and evaluate neoplasia 'hallmarks'. Results: Group 1: 45 $(92 \%)$ genes were elevated in NET transcriptomes; 50 genes $(98 \%)$ by qPCR (Fold change $>2, \mathrm{p}<0.05$ ). Gene expression was highly correlated $(\mathrm{p}<0.00001)$ between matched tissue and blood. Nine gene clusters were defined by $30(58 \%)$ genes; 7 clusters (proliferation, GF signaling, SSTR etc) were increased (FC $>2, p<0.05)$ in NET and closely correlated $(\mathrm{p}<0.0001)$ between tissue and blood. Merging cluster analysis and the MAAA identified significant differences in disease activity scores $(0-100 \%)$ between SD $(28.6 \pm$ $2.4 \%)$ and $\mathrm{PD}(83 \pm 3.2 \%, \mathrm{p}<0.0001)$. ROC AUC curve was $95 \pm$ $1.6 \%(\mathrm{p}<0.0001)$. Both SSA $(-26 \%, \mathrm{p}<0.05)$ and surgery $(-51 \%$, $\mathrm{p}<0.005)$ significantly reduced expression levels of six clusters. Conclusion: Measurements of blood transcripts reflect NET tissue values. Biologically relevant clusters differentiate disease stability and enable determination of tumor actiivty. Keywords: pcr. 


\section{H14}

\section{CA 19-9: A Novel Role in the Prognosis of Pancreatic Neuroendocrine Tumors}

\section{Mills L. ${ }^{a}$, Ramage J. ${ }^{\mathrm{b}}$, Quaglia A. ${ }^{\mathrm{c}}$, Basuroy R. ${ }^{\mathrm{a}}$,} Srirajaskanthan $R$. $^{\mathrm{d}}$

aENETS Centre of Excellence, Institute of Liver Studies, King's College Hospital, London, UK; b Department of Gastroenterology, Hampshire Hospitals NHS Trust, Basingstoke, UK; ' Department of Histopathology, Institute of Liver Studies, King's College Hospital, London, UK; ${ }^{\mathrm{d}}$ Department of Gastroenterology, University Hospital Lewisham, London, UK

Introduction: Prognostication is difficult for neuroendocrine tumours given their long survival. Staging, grading using Ki67 and receptor status are tools commonly used, but are not without their limitations. CA 19-9 is a tumour marker used in the diagnosis and follow-up of pancreatic adenocarcinomas despite multiple confounding factors, including jaundice. CA 19-9 is not useful in the diagnosis of PNETs having a sensitivity of only $19 \%$, but it is not known whether it has other roles in PNETs. Aim(s): To investigate the role of CA 19-9 in prognostication for PNETs. Materials and Methods: 103 PNET patients with serum CA 19-9 measured at diagnosis were studied. Histology was reviewed to confirm diagnosis and 3 cases of tumours with adenocarcinomatous features were excluded. Clinical notes were studied to identify jaundice, pancreatitis, cirrhosis and other parameters. Results: CA 19-9 was influenced by clinical jaundice $(p=0.007)$, digestive adenocarcinomas $(p=0.019)$ and Grade 3 histology $(\mathrm{p}<0.001)$. A multiple regression model was used to correct for these confounding variables $\left(\mathrm{p}<0.001, \mathrm{R}^{2}=0.25\right)$. After correction, elevated CA 19-9 levels were associated with a worse survival $(p=0.05)$. This association was most significant for Grade 3 tumours and it was verified that the effect was independent of Ki67 values (3 year survival 13\% vs. 74\%). Conclusion: CA 19-9 offers prognostic relevance for Grade 3 PNETs beyond that which can be achieved with Ki67. This result is independent of jaundice, Ki67, adenocarcinoma differentiation and tumour stage. Keywords: ca 19-9.

\section{H15 \\ High Thyrocalcitonin Serum Level in Neuroendocrine Tumors: Characteristics, Prognosis and Interest in Follow-Up?}

Nozière C. ${ }^{a}$, Chardon L. a , Lombard-Bohas C. ${ }^{a}$, Goichot B. ${ }^{b}$,

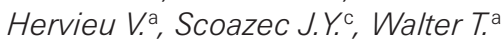

aHospices Civils de Lyon, Hôpital Edouard Herriot, Lyon, France; ' University Hospital, Endocrinology, Strasbourg, France; 'Hospices Civils de Lyon, Hôpital Edouard Herriot, France, France

Introduction: Thyrocalcitonin (TCT) is usually associated with medullary thyroid cancer. However, some neuroendocrine tumors (NETs) have also high serum TCT levels. Aim(s): The aims of this retrospective study were to assess a) characteristics and prognosis for
NETs producing TCT, and b) if this marker is useful for the follow-up of these tumors. Materials and Methods: All patients with NETs and whose serum TCT was determined between 2010 and 2012 were included. Clinical, biological, and histological characteristics were studied. Previous determinations of TCT were checked in patients with high TCT, defined as TCT $>100 \mathrm{ng} / \mathrm{L}$, and their levels were compared with biological and morphological follow-up. Results: Twenty-one of 176 patients with NETs (12\%) had high TCT, who were associated with bronchial and pancreatic NET $(\mathrm{p}<0.0001)$ and high grade tumors $(\mathrm{p}=0.0006)$. Medullary thyroid carcinomas (MTC) were excluded. TCT levels were not link with CEA levels. TCT staining by immunochemistry was shown in $5 / 6$ of high TCT NETs. Patients with high TCT levels, poorly diffentiated and grade 3 NETs were associated with poor prognosis after univariate analysis, but only the grade remains significant after multivariate analysis. Among the 22 patients with high TCT, 9 have functioning tumors: carcinoid syndrome $(n=6)$, VIPoma syndrome $(n=2)$ or Cushing syndrome $(n=1)$. Data on TCT changes during follow-up will be presented during the meeting. Conclusion: High TCT levels should also evoke a NET after excluding MTC, especially from a foregut primary. Keywords: thyrocalcitonin.

\section{H16 \\ Identification of Response Predictors to Temozolomide-Based Chemotherapy}

\author{
Strosberg J. ${ }^{\text {, }}$ Cives M. ${ }^{\text {, }}$ Brelsford M. ${ }^{\text {, }}$ Black M. ${ }^{\text {, }}$ \\ Meeker A. ${ }^{\mathrm{b}}$, Ghayouri M. ${ }^{\mathrm{a}}$ \\ ${ }^{a} \mathrm{H}$. Lee Moffitt Cancer Center and Research Institute, \\ Tampa, Fla., USA; bJohns Hopkins Kimmel Cancer Center, \\ Baltimore, Md., USA
}

Introduction: Capecitabine and temozolomide are active in the treatment of metastatic pNETs, with response rates ranging from $30 \%$ to $70 \%$. Several small retrospective series have suggested that MGMT deficiency may predict response to temozolomide, however expression of MGMT has not been validated as a predictive biomarker. Cytotoxic chemotherapy is thought to be most active in aggressive tumors, however the ki-67 index has not been formally evaluated as a predictive factor. It is unclear whether chromosomal instability (which correlates with alternate lengthening of telomeres) predicts response. $\operatorname{Aim}(\mathbf{s})$ : To identify predictive biomarkers of response to cape/tem in patients with pNETs. Materials and Methods: 144 patients with pNET who underwent treatment with cape/tem were retrospectively evaluated for radiographic response. The predictive role of ki-67\% and MGMT by IHC as well as ALT activation by FISH was assessed on up to 59 evaluable archival specimens. Frequently altered genes in pNETs were also sequenced and their status was correlated to the radiographic response. Results: The ORR was $63 \%$. Response to treatment was significantly higher in tumors with ki-67>5\% $(\mathrm{n}=28)$ as compared to tumors with ki-67 $\leq 5 \%(n=31)($ ORR: $64 \%$ vs. $29 \%$; $\mathrm{p}=0.006)$. MGMT status $(\mathrm{p}=0.358)$ and ALT pathway activation $(\mathrm{p}=0.174)$ were not predictive of response. Conclusion: pNETs with ki-67 $>5 \%$ are more likely to respond to cape/tem. MGMT status appears to have no correlation with response. Keywords: ki67, mgmt, alt, chromosomal instability, temozolomide, capecitabine, pnet. 


\section{Imaging (Radiology, Nuclear Medicine, Endoscopy)}

\section{I1 \\ Characterisation, Measurement and Biodistribution of an Improved Formulation of [Lu-177]-Octreotate}

Bailey D. ${ }^{a}$, Willowson K. ${ }^{b}$, Hennessy T. ${ }^{\text {, }}$ Schembri G. ${ }^{a}$, Snowdon G. ${ }^{a}$, Aslani A. ${ }^{a}$, Roach P. ${ }^{a}$, Pavlakis N. ${ }^{a}$, Clarke S. ${ }^{a}$

${ }^{a}$ Royal North Shore Hospital, Sydney, Australia; bUniversity of Sydney, Sydney, Australia

Introduction: Lu-177 can be made with high specific activity and no other isotopes of lutetium present, referred to as 'no carrier added' (nca) Lu-177. We have radiolabelled DOTA-conjugated peptide DOTA-(Tyr3)-octreotate (Auspep, Melb., AUS) with nca Lu-177 ('nca-LuTATE') and used it in over 30 administrations in NETs patients. Aim(s): Initial report on the accuracy, biodistribution and dosimetry of nca-LuTATE from gamma camera 2D whole body (WB) and quantitative 3D SPECT (qSPECT) Lu-177 imaging. Materials and Methods: Ten patients received $>30$ nca-LuTATE injections made with an E\&Z PharmTracer automated module. Extensive WB planar and qSPECT imaging was validated in vivo at $0.5,4,24$ and $96 \mathrm{hrs}$ to permit estimates of radiation dose using MIRD-based methodology (OLINDA-EXM). Results: WB planar images estimated radioactivity to within $4.2 \pm 7.0 \%(\mathrm{~N}=25)$ of the known total administered amount. Bi-exponential modelling of clearance showed $t^{1} / 2$ for the fast \& slow components of $2.1 \pm 0.6 \mathrm{hr}$ and $58.1 \pm 7.2 \mathrm{hr}$ respectively. qSPECT-estimated radioactivity concentration was accurate to within $-6.1 \pm 2.7 \%(\mathrm{~N}=6)$. The average dose to kidneys was $3.1 \pm$ 1.0 Gy per cycle. In 5 patients completing all 4 treatment cycles the average total dose to kidneys was $12.0 \pm 3.9 \mathrm{~Gy}$. Conclusion: We have shown that nca-LuTATE has an acceptable radiation safety profile and is a suitable alternative to previous carrier-added Lu-177 formulations. In addition, it has less radioactive waste issues and requires less peptide per treatment. Keywords: lutetium, dosimetry, biodistribution, effective dose.

\section{2}

\section{Signs of Carcinoid Heart Disease on Staging CT: Which Predictors Are Helpful?}

Baur A., Kunz F., Pschowski R., Röpke T., Pavel M., Denecke T.

\section{Charité-Universitätsmedizin Berlin, Berlin, Germany}

Introduction: In patients with functionally active neueroendocrine neoplasms (NEN) carcinoid heart disease presenting primarily with tricuspid valve regurgitation (TR) is associated with high morbidity and mortality. Early diagnosis is crucial to improve progno- sis. Aim(s): To evaluate signs on CT imaging performed for tumor staging as predictors of TR in patients with functionally active NEN. Materials and Methods: Patients with functionally active NEN and different grades of TR were identified. Two readers independently reviewed contrast-enhanced staging CT performed shortly before or after echocardiography. Logistic regression and receiver operating analyses were used to asses the predictive value of right ventricle to left ventricle ratio (RV/LV ratio), ventricular septal bowing, retrograde contrast filling of the hepatic veins during contrast injection, and time to aortal enhancement $>100 \mathrm{HU}$ during bolus tracking (TAE $>100$ HU) for TR. Results: Forty-four examinations were evaluated (11 with $\mathrm{TR}=$ grade 0,16 with $\mathrm{TR}=1,9$ with $\mathrm{TR}=2$, and 8 with $\mathrm{TR}=3$ ). $\mathrm{RV} / \mathrm{LV}$ ratio was found to predict $\mathrm{TR} \leq 1$ versus $\mathrm{TR}>1 \quad(\mathrm{p}=0.0188)$ and $\mathrm{TR} \leq 1$ versus $\mathrm{TR}=2(\mathrm{p}=0.0082)$. A prolonged TAE $>100 \mathrm{HU}$ predicted $\mathrm{TR} \leq 1$ versus TR $>1(\mathrm{p}=0.0077)$. AUC for $\mathrm{RV} / \mathrm{LV}$ ratio was 0.86 when differentiating $\mathrm{TR} \leq 1$ versus $\mathrm{TR}=2$. With a cut-off of 1.07 sensitivity was 0.89 , and specificity was 0.7 . Conclusion: In patients with functionally active NEN RV/LV ratio can predict TR with a relatively high sensitivity and moderate specificity. Keywords: computed tomography, staging, carcinoid heart disease.

\section{3}

A Comparative Study among Several Imaging Methods for the Detection of Primary and Metastatic Sites of GEP-NETs

Dimitroulopoulos D., Demiri M., Kypreos D., Malahias A., Theodoropoulos L., Hatzinikolaou M., Nikaki M., Stavrinidis S., Saribegioglou N., Tsamakidis K., Xinopoulos D.

Agios Savvas Cancer Hospital, Athens, Greece

Introduction: GEP-NETs staging are essential for any therapeutic manipulation. Aim(s): To evaluate the sensitivity of several imaging methods in detection of GEP-NETs and their metastases. Materials and Methods: 62 patients (48 confirmed GEP-NETs/14 suspected disease) were enrolled. All were investigated for primary and metastatic sites with somatostatin receptor scintigraphy (SRS), chest $-\mathrm{X}$ ray, upper abdominal US, chest CT and upper and lower abdominal CT. Results: SRS visualized primary or metastatic sites in $44 / 62(71.0 \%)$ patients with already known $(32 / 48-66.7 \%)$ or highly suspected disease (12/14-87.5\%). Conventional imaging was positive in $38(61.3 \%)$ patients $(8 / 14-57.1 \%$ under investigation and $30 / 48-62.5 \%$ with known tumors). SRS provided additional detection sites vs. conventional imaging (71.0\% vs. $61.3 \%)$. Detection rate of primary sites was higher with SRS than that of conventional imaging $(30 / 42-71.4 \%$ vs. $38.1 \%, P=0.039)$. SRS failed to detect primary tumors in $12(28.6 \%)$ patients, 8 with known lesions and 4 under investigation. Only 1 of these lesions was diagnosed with conventional imaging. The positive detection rate in metastatic sites was similar for SRS and conventional imaging ( $48.4 \%$ and $51.6 \%, \mathrm{P}>0.05)$. In the 38 patients with metastases, SRS failed to visualize these in $8(21.1 \%)$, all in liver. Conventional imaging visualized metastases in $32(84.2 \%)$ patients. Conclusion: SRS imaging is a sensitive method for the detection of GEP-NETs but is less sensitive than conventional imaging in the detection of liver metastases. Keywords: gep-net, staging. 
14

\section{CT Imaging Findings of Gastric Neuroendocrine Neoplasm According to 2010 WHO Classification}

\author{
Jiang L.M., Liang Z.W., Li Y., Geng C.Y., Shi S.S.
}

Cancer Hospital/Institute Chinese Academy of Medical

Science and Peking Union Medical College, Beijing, China

Introduction: Gastric neuroendocrine neoplasm (G-NEN) is rarely detected at an early stage. Its prognosis varies based on grade and stage. Aim(s): To describe CT findings of G-NEN according to 2010 WHO classification. Materials and Methods: 33 patients with histologically or pathologically documented G-NEN were included in this study. The lesions'number, location, shape, size, density, enhancement patterns and other changes on contrast-enhanced CT images were observed. Contrast-enhanced CT images of the tumors were reviewed between tumors different grading (NET, NEC). Results: G1 tumors were found in 12 patients, G2 in 2 patients, G3 in 18 patients, and mixed adenoneuroendocrine carcinoma in 1 patient. Marked enhancements were observed on CT scan. Tumor located at the gastric fundus and cardia part in 9 cases $(8 / 9$, NEC), greater curvature of stomach body in 9 cases $(6 / 9$, NET), the lesser curvature in 13 cases $(9 / 13, N E C)$, and antrum in 3 cases $(2 / 3, N E T)$. The tumor size was less than $3 \mathrm{~cm}$ in diameter in 13 cases (13/13, NET), and larger than $3 \mathrm{~cm}$ in 21 cases $(18 / 21$, NEC). Ulceration was observed in 17 cases $(15 / 17, \mathrm{NEC}) .15$ tumors showed intra-luminal growth $(12 / 15, \mathrm{NET})$ and 19 cases involved both intra and extra-luminal $(19 / 19$, NEC). Metastases were observed in 14 cases $(14 / 14$, NEC) $(\mathrm{P}<0.005)$. Conclusion: G-NEN are commonly hypervascular. CT imaging features correlates to the pathological grade. Thus, CT imaging findings may be beneficial in the evaluation of the tumor grade prior to resection or biopsy. Keywords: gastric neuroendocrine neoplasm, ct, pathological grade.

\section{5}

\section{The Additional Value of Somatostatin Receptor Scintigraphy during Preoperative Staging in Patients with Non-Functioning Pancreatic Neuroendocrine Tumors}

Jilesen A., Hoefnage/ S., Busch O., Bennink R., Gouma D., Nieveen van Dijkum $E$.

Academic Medical Center, Amsterdam, The Netherlands

Introduction: During diagnostic workup for non-functioning pancreatic neuroendocrine tumors (NF-pNET) both computed tomography scan (CT-scan) and somatostatin receptor scintigraphy (SRS) are made. Aim(s): To determine the additional value of the SRS in pre-operative diagnostic workup as second test after CT-scan. Materials and Methods: All SRSs from January 2002-July 2013 made in the Academic Medical Center of Amsterdam, the Netherlands were selected. Patients with NF-pNET were included if both CT-scan/ SRS were made during pre-operative workup. Diagnostic accuracy of the CT-scan and SRS was analysed as well as the percentage of changed TNM classification after SRS. Results: Overall, 62 patients with NF-pNET were included. Sensitivity for the primary tumor for CT-scan and SRS were resp. 95 and $73 \%$. Sensitivity and specificity for the CT-scan and SRS in the detection for metastases were resp. $85 \%-80 \%$ and $85 \%-90 \%$. The positive predictive value and negative predictive value for the CT-scan and SRS in the detection for metastases were resp. $52 \%-95 \%$ and $69 \%-96 \%$. In $21 \%$ of the patients, the TNM classification changed after SRS. Conclusion: In nonmetastatic NF-pNET on CT scan, the SRS had no additional value in staging. However, additional SRS is indicated to confirm suspicious lesions for NET-metastases. Keywords: somatostatin receptor scintigraphy, pancreatic neuroendocrine tumor, preoperative staging.

\section{6 CT Evaluation of Gastric Neuroendocrine Neoplasm Based on WHO 2010 Classification

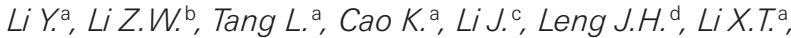
Sun Y.S. ${ }^{\mathrm{a}}$

aDepartment of Radiology, Peking University Cancer Hospital \& Institute, Beijing, China; 'bepartment of Pathology, Peking University Cancer Hospital \& Institute, Beijing, China; 'Department of Gastroenterology, Peking University Cancer Hospital \& Institute, Beijing, China; dDepartment of Hepato-Pancreatic Biliary Surgery, Peking University Cancer Hospital \& Institute, Beijing, China
\end{abstract}

Introduction: The 2010 WHO classification classifies GEPNEN into grade 1, 2 and 3. Aim(s): To investigate CT appearances in gastric neuroendocrine neoplasm (NEN) and their correlation with pathological grades based on WHO 2010 classifications. Materials and Methods: $\mathrm{CT}$ images of 34 patients with pathologically proven gastric NEN were retrospectively interpreted, including the detection rate, the shape, the thickness, the largest cross-sectional area, $\mathrm{T}$ and $\mathrm{N}$ stage of the lesion. The correlation between CT findings and pathological grades were analyzed. Results: Thirty six gastric NENs were detected in surgery and/or endoscopy. CT found 16 lesions (1 G1, $2 \mathrm{G} 2$ and $13 \mathrm{G} 3$ ) while missed 10 (4 G1 and $6 \mathrm{G} 2)$ in 26 neuroendocrine tumors/carcinomas (NET/NEC, 5 G1, 8 G2 and 13 G3). In 10 mixed adenoneuroendocrine carcinomas (MANEC, all G3), CT found 9 while missed 1. Gastric NEN appeared as small sessile polyps (3/26), ulcerations $(21 / 26)$ and diffuse thickening of the gastric wall $(1 / 26)$. The CT detection rate, thickness and the largest area, $\mathrm{T}$ stage and N stage in NEC (G3) group were significantly higher than those in NET (G1+G2) group (100\% vs. $23.1 \%, 20.23 \pm 13.46 \mathrm{~mm}$ vs. $0 \mathrm{~mm}, 1855.92 \pm 2658.28 \mathrm{~mm}^{2}$ vs. $18.33 \pm 2.08 \mathrm{~mm}^{2}$, T3 and T4 vs. T1, N1 vs. N0, respectively, $\mathrm{P}<0.05)$. Conclusion: The demonstration of gastric NEN on CT correlates with the pathological grades. $\mathrm{CT}$ can reveal the shape and size of the lesion, evaluate the $\mathrm{T}$ and $\mathrm{N}$ stage and may indicate the pathological grades, thus affect clinical management. Keywords: ct, gastric nen, pathological grade. 
17

\section{Role of Enteroclysis MDTC in the Diagnosis and Follow-Up of Ileal Neuroendocrine Tumors}

\author{
Maiettini D. a, Bellucci M.C. ${ }^{\text {a }}$,Valloncelli C. ${ }^{\text {a }}$ Scialpi M. ${ }^{\text {, }}$ \\ Rebonato A. ${ }^{\text {a }}$ Ferolla P. \\ aDepartment of Radiology, University of Perugia, \\ Santa Maria Della Misericordia Hospital, Perugia, Italy; \\ bDepartment of Medical Oncology, University of Perugia, \\ Santa Maria Della Misericordia Hospital, Perugia, Italy
}

Introduction: Among all the well differentiated neuroendocrine tumors (NET) those originating from ileum present the more relevant diagnostic difficulties and remain therefore occult in a relevant percentage of cases in all the stages. Aim(s): to evaluate the effectiveness of enteroclysis Multidetector-row Computed Tomography (E-MDCT) in the diagnosis of ileal NET. Materials and Methods: A consecutive series of 956 of E-MDCT and more than 850 NET patients were rewieved for the study. The clinical indications for E-MDCT were 1) presence of carcinoid syndrome 2) NET liver metastases 3) abdominal cramps; 4) bowel obstruction, recurrences; 5) GI bleeding. Results: CT-E identified 46 ileal NETs (43 new diagnosis and 3 recurrences). The primary tumor appeared as nodular or multifocal lesion $(n=27,59 \%)$ with an intense arterious contrast enhancement, with ulcers $(\mathrm{n}=16,35 \%)$ or hemorragic wall $(\mathrm{n}=11$, $24 \%$ ). Size range was 4 to $30 \mathrm{~mm}$. Liver and lymphnode metastasis were identified in $32(70 \%)$ and $43(93 \%)$ patients respectively. Other radiological signs were: 'hairpin turn' sign $(\mathrm{n}=33,72 \%)$; desmoplastic reaction $(\mathrm{n}=34,74 \%)$; 'sun burst' sign $(\mathrm{n}=19,41 \%)$ and lymph nodal calcification $(\mathrm{n}=24,52 \%)$. Carcinosis and ascites were identified in $7(15 \%)$ and $11(24 \%)$ patients, respectively. E-MDCT accuracy in identifying ileal NETs was 100 . Conclusion: E-MDCT is a reliable modality in the diagnosis and staging of small and multicentric carcinoids of the ileum, and in follow-up for suspected recurrence or metastatic disease. Keywords: ileal neuroendocrine tumor, enteroclysis ct.

\section{8 \\ Functional Imaging Tests versus Computed Tomography Scan: Detection of New Metastases and Clinical Usefulness in Digestive Neuroendocrine Neoplasms Follow-Up}

Merola E. a, Panzuto F. , Capurso G. ${ }^{\mathrm{a}}$, Kump P. ${ }^{\mathrm{b}}$, Lipp R. ${ }^{\mathrm{C}}$, Cicchese N. ${ }^{a}$, lannicelli E. ${ }^{\mathrm{d}}$, Prosperi D. ${ }^{\mathrm{e}}$, Pizzichini P. Rinzivillo M. ${ }^{a}$, Partelli S. ${ }^{\dagger}$, Rinke A. ${ }^{\mathrm{g}}$, Falconi M. ${ }^{\dagger}$, Delle Fave $G^{\text {a }}$

aDigestive and Liver Diseases Unit, Sant'Andrea Hospital (Sapienza University of Rome), Rome, Italy; 'Division of Gastroenterology and Hepatology, Department of Internal Medicine, Medical University of Graz, Graz, Austria; 'Division of Nuclear Medicine, Department of Radiology, Medical University of Graz, Graz, Austria; dDepartment of Radiology, Sant'Andrea Hospital (Sapienza University of Rome), Rome, Italy; eDivision of Nuclear Medicine, Sant'Andrea Hospital (Sapienza University of Rome), Rome, Italy; ${ }^{f}$ Pancreatic Surgery Unit, University Hospital of Ancona, Ancona, Italy; ' Department of Gastroenterology, University Hospital of Marburg, Marburg, Germany

Introduction: Digestive Neuroendocrine Neoplasms (DNENs) express in $80 \%$ of cases somatostatin receptors (SSTRs) detected by functional imaging tests (FITs). FITs are needed at diagnosis to define therapy but their role in follow-up (FU) is unclear. Aim(s): To compare accuracy in detecting new lesions (primary outcome) and clinical usefulness (secondary outcome) of FITs and Computed Tomography scan (CT) in stage IV DNENs FU. Materials and Methods: Retrospective analysis of stage IV DNENs expressing SSTRs with at least 24 month-FU. From 1995 to 2008 FIT was Octreoscan (OCT), from 2008 to 2013 68Ga-DOTANOC PET (GaPET). CT was repeated every 6-12 months, FIT yearly. FU time was divided in 12 month-'units' (in which pts had at least 1 CT and 1 FIT), analyzed separately and compared to each other. Gold standard was the result of imaging, surgical and pathology findings collected for each pt in FU. Clinical usefulness was defined as appropriate changes in management (new imaging, therapy, surgery, biopsy) due to CT or FITs. Results: 323 units (median 3 per pt, range 2-7) from 99 pts. New lesions were detected by CT in $86.4 \%$ of cases (PPV 93.3\%, NPV 91.9\%); adding OCT diagnostic yield was $10.34 \%$ (P < 0.05; PPV 100\%, NPV 98.8\%;), with GaPET 21.7\% (P < 0.05; PPV 100\%, NPV $100 \%$ ). Appropriate changes were due to CT in $84.9 \%$ of cases; adding OCT diagnostic yield was $11.6 \%(\mathrm{P}<0.05)$, with GaPET $33.3 \%$ $(\mathrm{P}<0.05)$. Conclusion: Addition of FIT to CT during DNENs FU significantly improves detection of new lesions and clinical management. Keywords: metastatic, dnens, follow-up, fit, ct. 
19

\section{Role of Combined 68Ga-DOTATOC and 18F-FDG PET-CT in the Diagnostic Workup of Well Differentiated Pancreas Neuroendocrine Tumors (PanNETs): A Surgical Series}

Ortolani S. ${ }^{a}$, Butturini G. ${ }^{\mathrm{b}}$, Malpaga A. ${ }^{\mathrm{b}}$, Cingarlini S. ${ }^{\mathrm{a}}$ Malfatti V. ${ }^{c}$, Capelli P. ${ }^{\mathrm{d}}$, Ruzzenente A. ${ }^{\mathrm{e}}$, Regi $\mathrm{P}^{\mathrm{f}}$, Vallerio P. ${ }^{\mathrm{g}}$, Grego E. ${ }^{a}$, Tortora G. ${ }^{\text {, }}$ Scarpa A. ${ }^{\mathrm{d}}$, Bassi C. ${ }^{\mathrm{b}}$, Salgarello M. ${ }^{\mathrm{c}}$

${ }^{a}$ Comprehensive Cancer Network, G.B. Rossi University Hospital-Oncology Department, Verona, Italy;

${ }^{b}$ Comprehensive Cancer Network, G.B. Rossi University Hospital-Pancreatic Surgery Department, Verona, Italy; 'Sacro Cuore Don Calabria Hospital-Nuclear Medicine Department, Negrar (VR), Italy; 'Comprehensive Cancer Network, G.B. Rossi University Hospital-Pathology Department, Verona, Italy; ${ }^{e}$ Comprehensive Cancer Network, G.B. Rossi University Hospital-Hepato-Biliary Surgery Department, Verona, Italy; ${ }^{f}$ Casa di Cura PederzoliPancreatic Surgery Department, Peschiera del Garda (VR), Italy; ' ${ }^{9}$ Comprehensive Cancer Network, G.B. Rossi University Hospital-Internal Medicine Department, Verona, Italy

Introduction: PanNETs have a broad spectrum of aggressiveness. Somatostatin-receptor based functional imaging, [68Ga] DOTATOC-PET/CT (GP), is recommended in the diagnostic workup of PanNETs; preliminary evidences show that [18F]FDG-PET/CT (FP) can provide prognostic information. Aim(s): To assess the role of combined GP and FP in the evaluation of G1-2 PanNETs and to test the correlation between FP positivity and tumor grade. Materials and Methods: Preoperative GP and FP of 35 patients with surgically resected G1-2 PanNETs were evaluated. Correlation between FP positivity (SUV $\max >3.5$ ) and tumor grade was assessed. Results: Of 35 surgically resected PanNETs, 10 (28.6\%) were G1 and 25 (71.4\%) were G2. GP showed high sensitivity (94.3\%) in detecting G1-2 PanNETs. FP resulted positive in $2 / 10(20 \%)$ and $19 / 25(76 \%) \mathrm{G} 1$ and G2, respectively. Therefore, FP was able to identify G2 pNETs with high positive predictive value (PPV, 90.5\%). Conclusion: The high sensitivity of GP in NET detection is well recognized. The high PPV of FP in the identification of G2 forms suggests its potential role in risk stratification for PanNETs. The complementary information provided by FP may be relevant in the decision-making for small $(<2 \mathrm{~cm})$ potentially resectable PanNETs. Given the reliability of FP correlation with tumor grade determined on surgical specimens, FP could be proposed in the diagnostic workup of PanNETs to target the bioptic sample on FP positive areas and to guide the interpretation of bioptic diagnosis. Keywords: pancreas neuroendocrine tumor, pet, tumor grade.
110

Perfusion Changes in Liver Metastases (LM)
from Pancreatic Neuroendocrine Tumors
(PanNETs) during Everolimus (E) Treatment:
Update of Perfusion CT (P-CT) Study

Ortolani S. ${ }^{a}$, De Robertis R. ${ }^{b}, D^{\prime}$ Onofrio M. ${ }^{b}$, Cingarlini S. ${ }^{a}$, Crosara S. ${ }^{\text {, }}$ Butturini G. ${ }^{c}$, Ruzzenente A. ${ }^{\text {d }, ~ D a v i ~ M . V . ~}{ }^{\mathrm{e}}$, Vallerio P. ${ }^{\mathrm{e}}$ Trentin C. ${ }^{\text {, }}$ Tortora G. ${ }^{\text {a }}$ Scarpa A. ${ }^{\dagger}$, Bassi C. ${ }^{\mathrm{c}}$, Pozzi Mucelli R. ${ }^{\mathrm{b}}$

${ }^{a}$ Comprehensive Cancer Network, G.B. Rossi

University Hospital-Oncology Department, Verona, Italy; ${ }^{b}$ Comprehensive Cancer Network, G.B. Rossi University Hospital-Radiology Department, Verona, Italy; ${ }^{\mathrm{C}}$ Comprehensive Cancer Network, G.B. Rossi University Hospital-Pancreatic Surgery Department, Verona, Italy; ${ }^{\mathrm{d} C o m p r e h e n s i v e ~ C a n c e r ~ N e t w o r k, ~ G . B . ~ R o s s i ~ U n i v e r s i t y ~}$ Hospital-Hepato-biliary Surgery Department, Verona, Italy; e Comprehensive Cancer Network, G.B. Rossi University Hospital-Internal Medicine Department, Verona, Italy; ${ }^{f}$ Comprehensive Cancer Network, G.B. Rossi University Hospital-Pathology Department, Verona, Italy

Introduction: P-CT can evaluate inner perfusional features of primary and metastatic PanNETs; preliminary evidences suggest its potential role in predicting early response to anti-angiogenic therapies. Perfusional modifications induced by E in LM are not completely understood. Aim(s): To evaluate modifications detected by P-CT in LM from PanNETs during E treatment. Materials and Methods: Seven patients with LM from G1-2 PanNETs were prospectively evaluated. P-CT was performed at baseline (T0), after 2 (T1) and 4 (T2) months of E. Lesion size, blood flow (BF), blood volume (BV), peak enhancement intensity (PEI) and time to peak (TTP) were calculated. Comparison between P-CT features at different time points was performed with 2-tailed unpaired Student's T test. Results: Eighteen LM were evaluated; $72.2 \%$ LM showed size stability during E treatment. Twelve LM (66.6\%) had a significant overall decrease in BF $(p=0.002)$, while the remaining $6(33.4 \%)$ had a significant increase $(\mathrm{p}=0.007)$. BV significantly increased from T0 to T2 in $10(55.5 \%)$ LM $(\mathrm{p}=0.002)$; the remaining $8 \mathrm{LM}$ showed a significant BV decrease $(\mathrm{p}=0.007) .83 .3 \%$ LM showed a significant PEI decrease (from T0 to T2, p = 0.001), even at an early stage (from $\mathrm{T} 0$ to $\mathrm{T} 1, \mathrm{p}=0.02$ ). TTP was not significantly modified by $\mathrm{E}$. Conclusion: E treatment seems to decrease BF and PEI and increase BV in PanNETs LM. Size and TTP are not modified by E. Longer follow up is needed to study the correlation between perfusional modifications and clinical outcomes. Keywords: perfusion ct, pancreas neuroendocrine tumor, everolimus. 
111

\section{Contrast Harmonic Endoscopic Ultrasonography (CH-EUS) Is Able to Predict Aggressivity in Pancreatic Neuroendocrine Tumors}

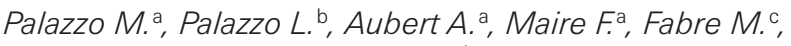

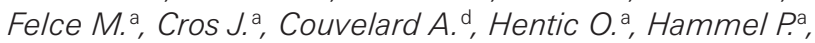 \\ Lévy P. ${ }^{a}$ Ruszniewski P.

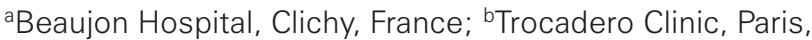 \\ France; 'Gustave Roussy Institute, Villejuif, France; \\ dBichat Hospital, Paris, France
}

Introduction: Pancreatic neuroendocrine tumors (PNET) are a heterogeneous group regarding malignancy. Aim(s): To assess the ability of CH-EUS to predict aggressivity in PNET. Materials and Methods: In a monocentric retrospective study, histologically proven PNET were included and classified in two groups: " lowly aggressive »/«highly agressive» tumors corresponding to T3-T4 and/ or $\mathrm{N}+$ and/or $\mathrm{M}+\mathrm{G} 1 / \mathrm{G} 2$, or G3. Uni- and multivariate analyses were performed to assess factors associated with aggressivity. Sensitivity (Se), Specificity (Sp), Positive Predictive Value (PPV) and Negative Predictive Value (NPV) were calculated. Results: 92 tumors (62 « lowly aggressive » and 30 " highly aggressive ») were included. 32 tumors had hypoenhanced areas at $\mathrm{CH}$-EUS. At univariate analysis, size, irregular margins, attenuation of the ultrasound beam, Ki67 proliferation index and hypoenhancement at CH-EUS were predictive of aggressivity. At multivariate analysis, only hypoenhancement remained significant. Se, Sp, PPV, NPV of hypoenhancement at CH-EUS was $93.3 \%, 93.5 \%, 87.5 \%$ and $96.7 \%$. Of $26 \mathrm{G} 1,<20$ $\mathrm{mm}$ non-functioning tumors, 8 were aggressive. In this subgroup, Se, $\mathrm{Sp}, \mathrm{PPV}, \mathrm{NPV}$ of hypoenhancement at CH-EUS for the diagnosis of aggressivity were $87.5 \%, 94.4 \%, 87.5 \%$ and $94.4 \%$. Conclusion: CH-EUS, showing hypoenhanced areas in PNET, is able to predict aggressivity especially in $\mathrm{G} 1,<20 \mathrm{~mm}$ non-functioning tumors, and could be helpful for treatment decision, where surgical resection has to be carefully weighted against surgical risk. Keywords: pancreatic neuroendocrine tumor, ch-eus.
112 Ga-68 Somatostatin Receptor PET/CT in the
Detection of Insulinoma

Prasad V. ${ }^{\mathrm{a}}$, Blankenstein O. ${ }^{\mathrm{b}}$, Plöckinger U. ${ }^{\mathrm{c}}$, Pape U.F. Pascher A. e, Pavel M. ${ }^{d}$

aDepartment of Nuclear Medicine, Charité

Universitätsmedizin Berlin, Berlin, Germany;

bInstitute for Experimenteal Pediatric Endocrinology,

Charité Universitätsmedizin Berlin, Berlin, Germany;

Interdisziplinäre Stoffwechsel-Centrum, Department

of Hepatology and Gastroenterology, Charité

Universitätsmedizin Berlin, Berlin, Germany; dDepartment of Hepatology and Gastroenterology, Charité

Universitätsmedizin Berlin, Berlin, Germany; ' Department of General, Visceral and Transplant Surgery, Charité

Universitätsmedizin Berlin, Berlin, Germany

Introduction: Presence of somatostatin receptors on beta cells of pancreas is a matter of debate. $\mathbf{A i m}(\mathbf{s})$ : This retrospective study aimed at analysing the role of Ga-68 somatostatin receptor PET/CT (3-phase) in insulinoma patients (pts). Materials and Methods: We looked at the Ga-68 somatostatin receptor (SR) PET/CT (SR-PET) examination in pts with either suspicion of or histologically confirmed insulinomas between 01/2008-11/2014. Diagnosis was based on either histopathology and/or biochemical assessment. Results: 17 pts (median age 51 years; Female: Male 12:5), were found to fullfill the analyses criteria. Based on pathology (13/17) as the gold standard and biochemical follow-up $(\mathrm{N}=4), 4$ pts had malignant insulinoma, 2 adult nesidioblastosis and 9 benign insulinomas. 4 pts had MEN-1 syndrome. Ki67 ranged between 1-20\%. SR-PET was positive for somatostatin receptor positive lesions in 14/15 (93.3\%) pts and false negative in $1 / 15$ patients $(6.7 \%)$. In the benign insulinoma pts and nesidioblastosis, SR-PET could correctly detect the focus for insulin production in 10/11 (91\%) pts and was false negative in $1 / 11(9 \%)$. Based on biochemical and clinical follow-up SR-PET was truly negative in 2 pts. SR-PET results alone lead to change in surgical management in 5/9 (55\%) of benign insulinoma pts. Conclusion: SR PET/CT appears to be a highly sensitive for the detection of insulinoma and PET may lead to changes in management of insulinoma pts. These results need to be confirmed in a higher number of patients. Keywords: somatostatin receptor pet/ct, insulinoma. 
113

\section{Prognostic Role of FDG-PET in Advanced Digestive Neuroendocrine Neoplasms}

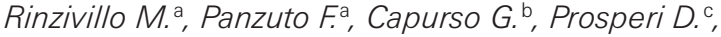 \\ Pizzichini P. , Cicchese N. a, Merola E. a, Schillaci O.d, \\ Scopinaro $F^{c}$, Delle Fave $G{ }^{b}$
}

aDigestive and Liver Disease Unit, Sapienza University of Rome, Rome, Italy; ${ }^{b}$ Digestive and Liver Disease Sapienza University of Rome, Rome, Italy; ' Department Nuclear Medicine, Rome, Sapienza University of Rome, Rome, Italy; 'Department Nuclear Medicine, Rome, Tor Vergata University of Rome, Rome, Italy

Introduction: The role of FDG-PET to predict clinical outcome in advanced digestive neuroendocrine neoplasms (dNENs) is still unclear. Aim(s): To determine the ability of FDG-PET to predict disease progression (DP) in patients with advanced dNENs. Materials and Methods: Retrospective analysis of prospectively enrolled consecutive patients with histologically confirmed advanced unresectable dNENs. PFS analysis was performed by Cox proportional hazards model. Results: 57 patients evaluated $(49.1 \%$ male; median age 59.5). Most frequent primary tumor sites were pancreas $(59.6 \%)$ and small bowel (33.5\%). Median Ki67 value was 7\% (24.5\% of patients had G1 tumors, $54.3 \%$ had G2 and 21\% had G3). $63.2 \%$ of patients $(\mathrm{n}=36)$ had positive FDG-PET, median SUV being 4.7. Of these, at time of FDG-PET examination, 56.1\% had a progressive disease, while $7 \%$ had a stable disease $(p=0.0001) .36 .8 \%$ of patients $(n=21)$ had negative FDG-PET; of these, at time of FDG-PET examination, $24.5 \%$ had a SD, while $12 \%$ had a PD $(p=0.05)$. Significantly longer median PFS was observed in FDG-PET negative patients, in comparison with FGD-PET positive patients (18 months vs. 9 months, respectively; $p<0.001$ ). Grading and FDG-PET positivity were independent predictors for tumor progression at multivariate analysis $(\mathrm{HR}=2.7$ $95 \%$ CI $1.55-4.7, \mathrm{p}=0.001$, and $\mathrm{HR}=3.595 \%$ CI $1.98-10.6, \mathrm{p}=$ 0.0003 respectively). Conclusion: FDG-PET should be considered in the diagnostic approach of patients with advanced dNENs in order to better predict the risk of disease progression. Keywords: fdg-pet, neuroendocrine neoplasms.

114

Small Bowel Neuroendocrine Tumors (NET): Relationship between Radiologic Features, Functionality and Ki-67 Classification

Rodriguez-Laval V., Tischer E., Pavel M., Wiedenmann B., Hamm B., Denecke T.

\section{Charité-Universitätsmedizin, Berlin, Germany}

Introduction: Since small bowel NETs are usually small tumors, detection and characterization of metastatic affection plays an important role. Aim(s): To analyze the correlation between small bowel NET radiological features with functionality and Ki-67. Materials and Methods: 75 consecutive pathologically proven small bowel NET were retrospectively studied with MRI/CT imaging. 2 expe- rienced radiologists in consensus analyzed radiological findings (metastatic pattern, tumor load, mesenteric vascular encasement and desmoplastic mass) and compared these with grading and functionality. Results: A dominant lymphatic or hepatic metastatic pattern was not associated with grading or functionality. $64 \%$ of the cases with medium or high hepatic metastatic load had G2-NET. 38\% of the patients with hepatic metastases had cystic metastatic lesions and featured also a high hepatic tumor load (84\%) and dissemination (71\%). There was not a significant difference between G1 and G2 in terms of lymphatic metastases with vascular encasement, but this was more frequently associated with liver metastases (with encasement $78 \%$ ) ( $p<0.05$ ); a desmoplastic mesenteric mass was in $82 \%$ (vs. $59 \%$ without fibrosis) associated with liver metastases and in $77 \%$ (vs. $38 \%)$ of the cases with carcinoid syndrome $(\mathrm{p}<0.05)$. Conclusion: G2-NETs tend to have cystic hepatic metastases, lymphatic metastases with vascular encasement and higher hepatic tumor load. A mesenteric desmoplastic mass was more frequent in functional NET, which suggests a causal correlation. Keywords: desmoplastic, ct, net, carcinoid.

\section{5 \\ Localisation of Insulinoma and Beta-Cells: Comparison of Glucagon-Like Peptide-1 Receptor (GLP1-R) SPECT/CT, PET/CT and MRI. Preliminary Results of a Prospective Clinical Study}

Rottenburger C. a, Antwi K. a, Heye T.a, Fani M. a, Nicolas G. a, Merkle E. a, Reubi J.C. ${ }^{b}$, Gloor B. ${ }^{c}$, Christ E. ${ }^{\text {, }}$ Wild D. ${ }^{\text {a }}$

aUniversity Hospital Basel, Clinic of Radiology \& Nuclear Medicine, Basel, Switzerland; 'bivision of Experimental Pathology, Department of Pathology, University of Bern, Bern, Switzerland; ' Department of Visceral Surgery, University Hospital of Bern, Inselspital, Bern, Switzerland; ${ }^{d}$ Division of Endocrinology, Diabetology and Clinical Nutrition, University Hospital of Bern, Bern, Switzerland

Introduction: SPECT/CT imaging with GLP1-R specific radiotracers like 111In-DOTA-exendin-4 have already proved to be a useful tool for the preoperative localisation of insulinomas. PET/CT has several advantages over SPECT/CT: lower radiation exposure, less time consumption and higher spatial resolution. $\operatorname{Aim}(\mathbf{s})$ : We aimed at prospectively comparing the detection rate of GLP1-R PET/CT vs. GLP1-R SPECT/CT vs. standardized contrast enhanced 3T MRI in patients with hyperinsulinemic hypoglycemia. Materials and Methods: Eleven adult Patients with neuroglycopenic symptoms due to endogenous hyperinsulinemic hypoglycemia were enrolled. Contrast media enhanced 3T MRI was performed. SPECT/CT imaging was performed with $111 \mathrm{In}-\mathrm{DOTA}$-exendin-4 and PET/CT with 68Ga-DOTA-exendin-4. Gold standard was the histological diagnosis. Results: Histology revealed benign insulinoma $(n=8)$ and adult nesidioblastosis $(n=1)$. In 1 patient all imaging modalities were negative and one patient refused surgery. MRI localised an insulinoma in 6, SPECT/CT in 7 patients. Only PET/CT identified one case of nesidioblastosis, 8 histologic proven cases of insulinoma and one case of focal uptake in a patient that refused surgery. Conclusion: In our 
study GLP1-R PET/CT performs better as standardized MRI imaging and GLP1-R SPECT/CT at lower irradiation doses and less time consumption, compared to SPECT/CT. The capability of GLP-1-R $\mathrm{PET} / \mathrm{CT}$ to localize adult nesidioblastosis needs further evaluation. Keywords: insulinoma, nesidioblastosis, glucagon-like peptide-1 receptor, pet/ct, spect/ct.

\section{6 \\ New Hybrid SPECT-CT Modality for Imaging Purposes in Patients with Medullary Thyroid Cancer (MTC)}

\author{
Sergieva S. ${ }^{a}$, Pandev R. ${ }^{\mathrm{b}}$, Robev B. ${ }^{\mathrm{a}}$, Fakirova A. ${ }^{\mathrm{c}}$ \\ aSofia Cancer Center, Sofia, Bulgaria; ' $U H^{\prime}$ Queen \\ Giovanna', Sofia, Bulgaria; 'Military Medical Academy, \\ Sofia, Bulgaria
}

Introduction: Loco-regional lymph node and distant metastases are found very often in patients with MTC of less than $1 \mathrm{~cm}$. There is not good MTC response to systemic chemotherapy or to external been radiotherapy. Radical thyroidectomy is the main method of therapy. Early diagnosis and correct N/M-staging are extremely important for the management of MTC. New hybrid SPECT-CT $\gamma$-cameras allow the fusion of anatomical and functional modalities. SPECT-CT is used in order to optimize somatostatin-receptor scintigraphic protocols. Aim(s): The aim of this study is to present advantages of hybrid somatostatin SPECT-CT images of MTC. Materials and Methods: 19 pts with MTC were studied: 5 for pre-treatment imaging and 14 with hypercalcitoninemia after surgical treatment for re-staging of the disease. SPECT-CT studies were performed 2-4 hrs post i.v.inj. of $740 \mathrm{MBq} 99 \mathrm{mTc}-$ Tektrotyd (Polatom), using SPECT-CT camera Symbia T2, Siemens. Results: In 5/5 pts for pre-treatment imaging, primary tumors were imaged -MTC. Loco-regional lymphadenopathy and distant pulmonary metastatic lesions were visualized in 2 of them. In the rest 14 pts SPECT-CT studies were used to evaluate the effect of surgical therapy: complete treatment response was obtained in 6 pts; progressive disease with local recurrence, cervical and bone metastases were established in 8 pts with hypercalcitoninemia. Conclusion: SPECT-CT studies have been reported to change clinical management in significant number of patients with MTC: surgery or radio/chemotherapy. Keywords: mtc, somatostatin, spectct image.
117

\section{Incremental Benefit of Preoperative Endoscopic Ultrasound for the Detection of Pancreatic Neuroendocrine Tumors: A Meta-Analysis}

\author{
Tsolakis A. ${ }^{\mathrm{a}}$, James P. ${ }^{\mathrm{b}}$, Zhang M. ${ }^{\mathrm{c}}$, Belletrutti P. ${ }^{\mathrm{c}}$, \\ Mohamed R. ${ }^{c}$, Roberts D. ${ }^{\mathrm{d}}$, Heitman S. ${ }^{\mathrm{c}}$ \\ aMedical Sciences, Uppsala University, Uppsala, Sweden; \\ bepartment of Medicine and The Ottawa Hospital \\ Research Institute, Ottawa, Canada; 'Department of \\ Medicine and Community Health Sciences, Calgary, \\ Canada; dDepartment of Surgery and Community Health \\ Sciences, Calgary, Canada
}

Introduction: Current guidelines recommend computed tomography (CT) scan or magnetic resonance imaging as the initial imaging modalities for the work-up of suspected pancreatic neuroendocrine tumors (PNETs). Aim(s): To determine the incremental benefit of preoperative endoscopic ultrasound (IBEUS) for the detection of suspected PNETs after other investigative modalities have been attempted. Materials and Methods: This systematic review searched MEDLINE, EMBASE, bibliographies of included articles, and conference proceedings for studies reporting original data regarding the preoperative detection of PNETs. Pooled IBEUS was calculated using random effects models. Heterogeneity was explored using stratified meta-analysis and meta-regression. Evidence of small study effects was assessed using funnel plots and Begg's test. Results: Among 4,505 citations identified, we included 17 cohort studies (612 patients). EUS identified PNETs in $97 \%$ of cases. Improved PNET identification with EUS was observed in all of the studies. After adjusting for small-study effects, meta-analysis showed that EUS alone could identify PNETs in approximately one in four patients (adjusted IBEUS 26\%, 95\% confidence interval 17-37\%). The pooled IBEUS varied based on the study design, study size, type of CT scan used and the number of modalities used prior EUS. Conclusion: Preoperative EUS is associated with an increase in PNET detection after other modalities are attempted. Keywords: pancreatic neuroendocrine tumor, radiological investigation, incremental benefit, endoscopic ultrasound. 
118

\section{Pre-PRRT Uptake on 68Ga-DOTATOC Predicts Good Prognosis in Neuroendocrine Tumors Patients}

Van Binnebeek S. a, Vanbilloen B. ${ }^{a}$, Baete K. ${ }^{a}$, Bogaerts K. ${ }^{\text {b, }}$ Koole M. ${ }^{\mathrm{a}}$, Mottaghy F. ${ }^{\mathrm{c}}$, Clement P. ${ }^{\mathrm{d}}$, Mortelmans L. ${ }^{\mathrm{a}}$, Haustermans K. ${ }^{\mathrm{e}}$, Nackaerts K. ${ }^{\dagger}$, Verbruggen A. g, Van Cutsem E. ', Verslype C. ${ }^{\mathrm{h}}$, Deroose C. $^{\text {a }}$

aNuclear Medicine, University Hospitals Leuven and Department of Imaging and Pathology, KU Leuven, Leuven, Belgium; 'b Department of Public Health and Primary Care (I-BioStat), KU Leuven, Leuven, Belgium; 'Department of Nuclear Medicine, University Hospital Aachen, Department of Nuclear Medicine, University Medical Center Maastricht, Aachen, Maastricht, Germany: dMedical Oncology, University Hospitals Leuven and Laboratory of Experimental Oncology, KU Leuven, Leuven, Belgium; 'Radiation Oncology, University Hospitals Leuven and Department of Oncology KU Leuven, Leuven, Belgium; fDepartment of Respiratory Diseases, Respiratory Oncology Unit, KU Leuven, University Hospitals Leuven, Leuven, Belgium; ' Laboratory of Radiofarmacy, KU Leuven, Leuven, Belgium; 'Division of Digestive Oncology, University Hospitals Leuven and Department of Oncology KU Leuven, Leuven, Belgium

Introduction: There is a growing need to identify PRRTresponders on an early time point during therapy. Aim(s): Can pretherapeutic uptake on 68Ga-DOTATOC PET predict outcome of PRRT? Materials and Methods: Forty-four NET patients were treated with 3 or 4 cycles of $1.85 \mathrm{GBq} / \mathrm{m}^{2} /$ cycle 90Y-DOTATOC up to a maximal kidney biological effective dose of $37 \mathrm{~Gy}$. A 68Ga-DOTATOC-PET/CT was performed before and 40 weeks after the first cycle of PRRT (PRRT1). Response was assessed by CT, 40 weeks after PRRT1; progression free survival (PFS) was based on follow up CT and clinical information. Three to six target lesions were prospectively chosen on PETbaseline and median SUVmax was assessed. The relationship between median SUVmax-baseline and PFS, Ki67-index and tumor grade was examined and ROC-analysis was performed among others. Results: At 40 weeks post PRRT1, stable and progressive disease were found in 23 and 21 patients, respectively. The median PFS was 14 months. A significant correlation was found between median SUVmax on PETbaseline and tumor grade $(\mathrm{p}=0.035, \mathrm{r}=-0.32)$; no significant correlation was found with Ki67-index ( $p=0.064, r=-0.29)$; PFS was significantly stratified using a median SUVmax of 12.4 on PETbaseline $(p=0.0235)$, a median PFS of 9.1 months (95\% CI, 1.7-13.9) was shown for the group with median SUVmax $\leq 12.4$ and 17.7 months $(95 \% \mathrm{CI}$, 9.3-21.4) if median SUVmax >12.4. ROC-analysis showed an area under the curve of 0.72. Conclusion: Median SUVmax of 12.4 on $68 \mathrm{Ga}$-DOTATOC-PETbaseline could be used as potential pre-therapeutic predictive factor for PRRT. Keywords: pet, response, prrt.
119

\section{An Intrapatient Comparison of 99mTc-HYNIC-TOC with 68Ga-DOTA-TATE for Imaging Abilities of Gastroenteropancreatic Neuroendocrine Neoplasms}

\author{
Yu J. ${ }^{\text {a }}$, Li J. ${ }^{\text {, }}$ Yang $Z$. $^{\text {a }}$ \\ aKey Laboratory of Carcinogenesis and Translational \\ Research (Ministry of Education), Department of \\ Nuclear Medicine, Peking University Cancer Hospital \& \\ Ins, Beijing, China; ' Key Laboratory of Carcinogenesis \\ and Translational Research (Ministry of Education), \\ Department of Internal Medicine of Digestive Tumor, \\ Peking Universit, Beijing, China
}

Introduction: Imaging with 68Ga-labled somatostatin analogs provides some advantages over SRS imaging, and has therefore recently become more poprlar. $\operatorname{Aim}(\mathbf{s})$ : The aim of this study was to compare the imaging abilities of the recently developed somatostatin analog, 99mTc-HYNIC-TOC with 68Ga-DOTA-TATE in gastroenteropancreatic neuroendocrine neoplasm patients. Materials and Methods: Approved by the Beijing cancer hospital ethics committee, thirty-four GEP-NEN patients (17 men, 17 women; age range, 23-75 y; middle age, $51 \mathrm{y}$ ) with histologically proven were enrolled in the study. The time interval between the studies using each tracer was less than two weeks. Results: Positive rate of 99mTc-HYNICTOC was $58.8 \%(20 / 34$, and it was not statisticallty different from 68Ga-DOTA-TATE $(70.6 \%, 24 / 34)$. But the latter was statistically higher than the former in the involed systems, especially lymph nodes, liver and skeleton. Minordiameter and SUVmax (Standardized Uptake Value, SUV) of 99mTc-HYNIC-TOC positive liver lesions were statistically higher than negative lesions. Conclusion: 68Ga-DOTATATE PET/CT imaging shows a significantly higher detection rate compared with $99 \mathrm{mTc}-\mathrm{HYNIC}-\mathrm{TOC}$ scintigraphy, especially small or structure-complex lesions. Keywords: neuroendocrine neoplasms, somatostatin receptor, octreotide, positron emission tomography.

\section{Medical Treatment - Chemotherapy}

\section{J1 \\ Efficacy of Topotecan in Pretreated Metastatic Neuroendocrine Carcinoma \\ Apostolidis L., Jäger D., Winkler E.C. \\ National Center for Tumor Diseases (NCT), Heidelberg, Germany}

Introduction: Therapeutic options for metastatic poorly differentiated extrapulmonary neuroendocrine carcinoma (NEC) after prior platinum-based chemotherapy are limited. Topotecan is an approved second line chemotherapy for small cell lung cancer (SCLC) which is often considered to show a biological behavior similar to NEC. 
$\operatorname{Aim}(\mathbf{s})$ : To analyze the efficacy of topotecan in pretreated metastatic NEC patients. Materials and Methods: We performed an updated retrospective analysis of all patients treated with topotecan for metastatic NEC who presented at our center between January 2005 and December 2013. Results: Out of 29 identified patients, best response to topotecan was a stable disease in 5 patients, partial remission in 2 , resulting in a disease control rate of $24.1 \%$. Of the remaining 22 patients, $13(44.8 \%)$ showed a progressive disease and $9(31.0 \%)$ died before radiologic response could be evaluated. Median progression free (PFS) and overall survival (OS) after start of topotecan was 2.1 and 3.9 months respectively. In the subgroup analysis, patients with unknown primary vs. those with known primary showed a significantly prolonged PFS of 4.2 vs. 1.9 months $(p=0.005)$ and OS of 6.4 vs. 2.6 months $(p=0.029)$. Conclusion: In this largest analysis of a single therapeutic regimen in pretreated metastasic NEC so far, topotecan shows only moderate antitumor activity, lower than reported for SCLC. However, activity of topotecan seems higher in patients with unknown primary. Keywords: neuroendocrine carcinoma, chemotherapy, topotecan.

\section{$\mathrm{J} 2$}

\section{First-Line Chemotherapy in Patients with Metastatic Gastroenteropancreatic Neuroendocrine Carcinoma (GEP-NEC): A Retrospective Analysis}

Bongiovanni A. ${ }^{a}$, Riva N. ${ }^{a}$, Monti M. ${ }^{\text {a }}$ lanniello A. ${ }^{\mathrm{b}}$, Pieri F. Oboldi D. ', Galassi R. ', Cavaliere D. ', Zaccaroni A. ', Tartaglia A. e , Lundedei V., Gardini A. ${ }^{\mathrm{d}}$, Amadori D. a', Ibrahim T.

a Osteoncology and Rare Tumors Center, Istituto Scientifico Romagnolo per lo Studio e la Cura dei Tumori (IRST) IRCCS, Meldola, Italy; ' Nuclear Medicine Unit, Istituto Scientifico Romagnolo per lo Studio e la Cura dei Tumori (IRST) IRCCS, Meldola, Italy; ' Pathology Unit, Morgagni-Pierantoni Hospital, Forli, Italy; ' Surgery Department Morgagni-Pierantoni Hospital, Forli, Italy; eEndocrinology Unit Morgagni-Pierantoni Hospital, Forli, Italy; ${ }^{f}$ Gastroenterology and Digestive Endoscopy Unit, Morgagni-Pierantoni Hospital, Forli, Italy

Introduction: Gastroenteropancreatic neuroendocrine neoplasia (GEP-NENs) are divided on the basis of Ki67 status into G1-G2 neuroendocrine tumors (NETs) (Ki67<20\%) and neuroendocrine carcinoma (NECs) $(\mathrm{Ki} 67>20 \%)$. NEC patients generally have a poor prognosis and the first choice treatment is chemotherapy. A cisplatin/etoposide combination is often used as NECs are considered clinically similar to small cell lung cancer (SCLC), but no literature data is available. There is some evidence to suggest that carboplatin may also be effective in metastatic SCLC. Aim(s): Assess the efficacy of these combination in mGEP-NEC patients. Materials and Methods: We retrospectively evaluated 20 cases of metastatic GEPNECs classified according to WHO 2010 and treated with cisplatin 25 $\mathrm{mg} / \mathrm{m}^{2}$ on days $1-3$ or carboplatin $300 \mathrm{mg} / \mathrm{m}^{2}$ on day 1 followed by etoposide $100 \mathrm{mg} / \mathrm{m}^{2}$ on days 1-3, every 21 days. Results: Of the 19 evaluable patients, 13 (68\%) obtained a partial response, 4 (21\%) had stable disease (SD), and 2 (11\%) showed disease progression. Median overall and progression-free survival was 13.5 months and 10.9 months, respectively, with a better outcome in patients with positive GA68PET/CT. G3-4 neutropenia was the most frequent side-effect. No difference in outcome was observed between patients treated with carboplatin or cisplatin. Conclusion: Platinum-based chemotherapy would appear to be active in this patient setting. Further prospective studies are needed to confirm these data and to identify predictive and prognostic factors. Keywords: gep-nec, chemotherapy.

J3

\section{Gemox or Alkylating Agents in Neuroendocrine Tumors? The Help of O6-Methylguanine-DNA Methyltransferase (MGMT) Status?}

\author{
Dussol A.S. a, Joly M.O. a, Hervieu V. , Forestier J. ", \\ Lombard-Bohas C. ${ }^{\text {a }}$ Cassier P. ${ }^{\mathrm{b}}$ Scoazec J.Y. ${ }^{\mathrm{c}}$, Walter T. ${ }^{\mathrm{a}}$

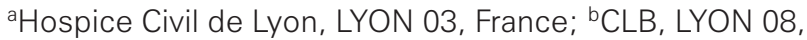 \\ France; 'IGR, Paris, France
}

Introduction: Alkylating agents are the backbone chemotherapy in neuroendocrine tumors (NET). However, gemcitabine associated to oxaliplatine (gemox) has showed interesting activity in a small study. $\operatorname{Aim}(\mathbf{s}):$ a) to confirm this data in a larger population, b) to compare its efficacy with alkylating agents, c) to explore if O6-methylguanineDNA methyltransferase (MGMT) status could help to choose between both types of chemotherapies. Materials and Methods: 96 patients with metastatic NET were treated with gemox (2004-2014), after a median of 2 other systemic treatments. Among these 96 patients, 57 also received an alkylating agent (ALK). Promoter gene methylation of MGMT was assessed by pyrosequencing in 23 patients treated with both chemotherapies. Results: After a median of 7 courses of gemox, 23\% had a partial response (PR). Median progression free survival (PFS) and overall survival (OS) were 7.9 months and 31.6 months respectively. Among the 57 patients who received both ALK and gemox, PR (23\% and 24\%) and PFS ( 7 and 8 months) were similar and $51 \%$ of patients had concordant response (PR/PR, stable/stable, or progressive/progressive) between both chemotherapies. There is a better outcome in methylated MGMT tumors of patients treated with ALK ( $p=0.03$ for PR and $p=0.08$ for PFS) but not with gemox. Conclusion: Gemox is effective in NET with similar activity than alkylating agents, but without influence of MGMT status. Prospective studies are needed, but ALK should be used in first-line against methylated MGMT tumors. Keywords: gemox, alkylating agent, mgmt. 


\section{J4 \\ TIM16 Inhibition Decreases Calcitonin Secretion and Enhances Sensitivity to Paclitaxel in a Human Medullary Thyroid Carcinoma Cells}

\author{
Gagliano T., Riva E., Tagliati F., Matteotti D., Brugnoli V., \\ Gentilin E., Guerrini R., Trapella C., Degli Uberti E., \\ Zatelli M.C. \\ University of Ferrara, Ferrara, Italy
}

Introduction: TIM 16 protein is part of the traslocase complex TIM 23 situated in the mitochondrial inner membrane, encoded by the Magmas gene, that is overexpressed in several tumors. Magmas silencing causes a greater sensitivity to apoptotic stimuli. We recently demonstrated that, in a human medullary thyroid carcinoma cell line (TT), compound 5, a TIM 16 inhibitor, was not cytotoxic but enhanced the proapoptotic effects of staurosporine. $\operatorname{Aim}(\mathbf{s})$ : To verify whether compound 5 influences calcitonin secretion and cytotoxic effect of paclitaxel. Materials and Methods: Cell viability and apoptotic activation were evaluated by ATPlite assay and Caspase 3/7 assay, respectively. ELISA test was used for calcitonin detection in cell culture medium. Results: $10 \mathrm{nM}$ paclitaxel was able to reduce cell viability by $40 \%$, while compound 5 alone had no effects. On the contrary, compound 5 was able to increase the effects of paclitaxel by nearly $14 \%$. Caspase $3 / 7$ activity was induced by paclitaxel $(+130 \%)$, but not by compound 5 , that, however, further potentiated the apoptotic effects of paclitaxel by $130 \%$. In addition we found that compound 5 was able to reduce basal and pentagastrin-induced calcitonin secretion. Conclusion: Compound 5 could represent a tool to increase the effects of chemotherapeutic agents and to control hypercalcitoninemia in medullary thyroid carcinoma. Keywords: tim16, magmas, paclitaxel, calcitonin, medullary thyroid carcinoma.

\section{J5 \\ Clinical Characteristics and Treatment Outcome of Six Advanced G3 Well-Differentiated Neuroendocrine Tumors}

\author{
Jia R., Chen H.E., Chen Y.L., Ge F.J., Liu R.R., Li S.S., \\ Zhao C.H., Liu L.J., Sun M.F., Zhang Y., Wang Y., Lin L., \\ Xu J.M.
}

Affiliated Hospital Cancer Center, Academy of Military Medical Sciences, Beijing, China

Introduction: According to 2010 WHO grading system, all neuroendocrine neoplasms were divided into G1/G2/G3 based on Ki-67 and mitotic count. However, differentiation was also very important for the biological behavior and treatment strategy. $\mathbf{A i m}(\mathbf{s})$ : This study focused on G3 well-differentiated neuroendocrine tumor (G3-WDNET), and demonstrated clinical characteristics and different treatment regimens for these patients. Materials and Methods: Differentiation of 43 G3 patients from January 2012 to November 2014 in our hospital were reexamined. Results: Six patients (14\%) with advanced disease were classified as G3-WDNET. All 6 patients were male and median age was 47 (25-59). Ki-67 was $25-60 \%$ and CgA was elevated in 2 out of 4 . NSE was elevated and SRS was positive in all 6 patients. Five patients had received chemotherapy including etoposide and cisplatin (EP) or temozolomide and capecitabine (CAPTEM), of them 4 had disease controlled for more than 7 months (the longest up to 2 years) including 2 partial response while one had disease progression after 2 cycles of EP and altered to CAPTEM; Three patients had received VEGFR TKIs in separate lines, of them 2 had disease progression after 1 cycle and the other had stopped treatment after 2 cycles due to various adverse events. Conclusion: G3-WDNET showed heterogeneous characters and varieties of treatment outcome. Patients who received chemotherapy might have better and longer disease control than those who received VEGFR TKIs. Keywords: neuroendocrine neoplasms, well differentiated g3, medical treatment.

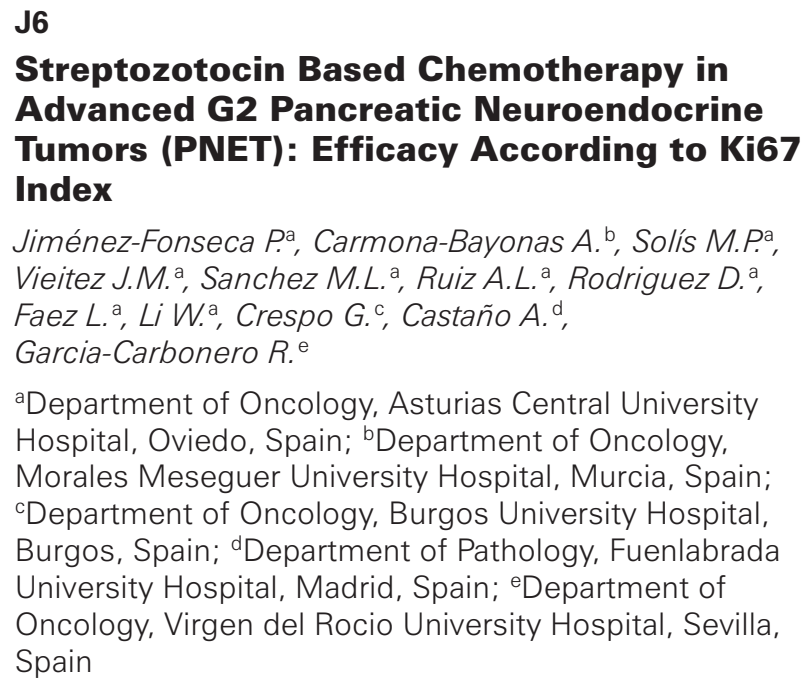

Introduction: Streptozotocin (STZ) with fluorouracil (FU) or doxorubicin (DOX) is approved in well differentiated advanced pancreatic neuroendocrine tumors (PNET). Aim(s): To evaluate the efficacy of STZ-FU and STZ-DOX in patients with grade 2 stage IV PNET according to Ki-67. Materials and Methods: We present a subset analysis from the RGETNE (registry of Spanish NET Group (GETNE)). Forty-five patients were recruited at a single Spanish centre from 2004-2012. Patients with Ki67 3-10\% received STZ-FU while those with Ki-67 11-19\% received STZ-DOX until progression. Response was evaluated according to RECIST v1.1 criteria every 3 months. Results: The median age was 66 years and $80 \%$ had ECOG 0 . Patients received a median of 13 cycles (range, 4-31). In patients treated with STZ-FU (Ki67 3-10\%) and STZ-DOX (Ki67 11-19\%), respectively, response rate was $21 \%$ and $56 \%$; disease control rate was $97 \%$ and $75 \%$; progression-free survival was 10 (95\% CI, 8.5-11.5) and 8.9 (95\% CI, 5.2-12.6) months; overall survival was 34 (95\% CI 26.9-41.1) and 25 (95\% CI 23.1-26.6) months. Grade 3-4 non-haematological toxicity was $6.6 \%(n=3)$. On Cox regression analysis, lower levels of Ki-67 (3-10\%) $(\mathrm{p}<0.01)$, obtaining a disease control $(\mathrm{p}=0.03)$ and longer time interval prior to progression $(\mathrm{p}=0.02)$ were predictors of survival. Conclusion: 
STZ-based chemotherapy is an effective and tolerable treatment in grade 2 PNET. Successful disease control was a predictor of overall survival together with the proliferation index Ki-67. Keywords: chemotherapy, streptozotocin, fluorouracil, pnet, ki67.

\section{J7 \\ Lack of Efficacy of Anthracycline-Containing Second Line Chemotherapy in Patients with GI-NEC after Cisplatin-Based Treatment}

Kiesewetter B. ${ }^{\text {, }}$ Koparek O. b, Mayerhoefer M.E. c, Niederle B. ${ }^{\mathrm{d}}$, Raderer M. ${ }^{\text {a }}$

aMedical University Vienna, Department of Medicine I/ Clinical Division of Oncology, Vienna, Austria; ${ }^{b}$ Medical University Vienna, Department of Pathology, Vienna, Austria; 'Medical University Vienna, Department of Radiology, Vienna, Austria; dMedical University Vienna, Department of Surgery, Division of General Surgery, Vienna, Austria

Introduction: Little is known about optimal 2nd-line treatment in patients with GI-NEC failing cisplatin-based therapy. Aim(s): As anthracycline-containing regimens including either doxorubicin or epi-doxorubicin plus vincristine and cyclophosphamide (ACO or EPICO) are commonly being used, patients have also been treated with these regimens at our institution. Materials and Methods: We have retrospectively analysed patients undergoing 2nd-line therapy after cis/eto. We identified 14 patients treated either with ACO $(\mathrm{n}=$ $2)$ or EPICO $(n=12)$. The majority of patients had metastatic NEC originating in the pancreas $(n=9)$, the colon $(n=2)$, and the stomach $(\mathrm{n}=1)$; two had unknown primary. The Ki-67 index was $25 \%$ 90\%; all patients had liver metastasis. Results: Tolerance of therapy was good, with only two patients experiencing haematological side effects grade III (leukocytopenia plus anemia and leukocytopenia, respectively), while treatment had to be delayed in one patient due to Pseudomonas infection occurring after two courses of therapy. Two patients developed mild vincristine associated peripheral neuropathy. However, no objective remissions were reported: 4 patients had stable disease lasting 3-7 m, while one patient progressed after one, two after two cycles, respectively, and 7 after 3 courses. Conclusion: Our retrospective analysis suggests that in spite of a low tox-profile, anthracycline-containing therapy is not active in patients with GI-NEC relapsing/progressing from 1st-line therapy with cis/eto, and should not be pursued further. Keywords: gi-nec.
J8

Unresectable Gastrointestinal
Neuroendocrine Liver Metastases
Treated by Liver-Directed Therapies

Liu L.J., Xin B., Ji Y., Yan Z., Lou W.

Fudan University Zhongshan Hospital, Shanghai, China

Introduction: Hepatic metastases develop in $85 \%$ of patients with GI neuroendocrine tumors. Liver-directed therapies allow for manageable microinvasive treatments able to improve the benefit and reduce the toxicity. $\mathbf{A i m}(\mathbf{s})$ : This study aimed to assess the feasibility, safety, efficacy and prognostic factors in patients with unresectable GI neuroendocrine liver metastases treated by liver-directed therapies including TACE and RFA. Materials and Methods: 24 patients (5 women) aged 28 to 77 years (mean age: $57 \mathrm{Y}$ ) histologically diagnosed GI NET-LM (14 cases were synchronous and 1 functioning tumor was gastrinoma) who were performed loco-regional therapies in our hospital from Jun 2004 to sep 2014 were analysed. Results: The tumors origin of prosogaster, midgut, hindgut were $45.8 \%, 16.7 \%, 37.5 \%$. The WHO standard G1, G2, G3 accounted for $16.7 \%, 37.5 \%, 45.8 \%$. $70.8 \%$ primary tumors were resected. The percentages of main vascular invasion, portal vein thrombus, lymph node metastases were $4.2 \%$, $12.5 \%, 41.7 \%$. All patients underwent $1-13$ TACE procedures (median 4) and $20.8 \%$ underwent 1 extra RFA. The rate of complete and partial response was $79.6 \%$. Overall survival rates of 1, 3, 5-year were $75 \%$, $66.7 \%, 62.5 \%$. The median survival time was 119-mo (3-204 mo). Higher grade $[\mathrm{P}=0.003, \mathrm{Ki}-67$ index $\geqq 10 \%(\mathrm{P}=0.025)$, the prosogaster origin $(\mathrm{P}=0.024)]$, and poor differentiation $(\mathrm{P}=0.008)$ were prognosis factors. Conclusion: The liver-directed therapies demonstrated its efficacy for unresectable metastatic hepatic GI-NETs. Keywords: neuroendocrine, tace, rfa, liver metastases, liver-directed therapy.

J9

Temozolomid-Based Therapy in GEP- and Bronchopulmonary NEN from a Multicentric Study from Germany

Maasberg S. ${ }^{a}$, Bacher M. ${ }^{a}$, Rinke A. ${ }^{b}$, Lahner H. ${ }^{c}$, Unger N. ${ }^{c}$, Quietzsch D. ${ }^{d}$, Begum N. ${ }^{\text {, Fottner C. }}$, Pavel M. ${ }^{\text {, }}$ Pape U.F. ${ }^{a}$

aMedical Department of Gastroenterology and Hepatology, University Medicine Charité, Berlin, Germany; 'bepartment of Gastroenterology and Endocrinology, University Hospital Gießen/Marburg, Campus Marburg, Marburg, Germany; ' Department of Endocrinology and Metabolism, University Hospital, Essen, Germany; ${ }^{d}$ Department of Internal Medicine, Clinical Centre of Chemnitz, Chemnitz, Germany; 'Department of Surgery, University Hospital Schleswig-Holstein, Campus Lübeck, Lübeck, Germany; fDepartment of Endcrinology and Metabolism, University Medicine, Mainz, Germany

Introduction: Chemotherapy (CTx) with temozolomid as mono (TEM) or combination therapy with capcitabine (TEM/CAP) is a new cytotoxic regimen with good efficiency in pancreatic and bronchopul- 
monary NET-G1/G2 and NEC-G3. Aim(s): Therefore TEM-based CTx was analyzed in a multicentric study from the German NETregistry. Materials and Methods: Patients (pts.) diagnosed since 1988 and treated with TEM or TEM/CAP were included, using SPSS 19.0 for analysis. Results: 66 pts. with median age of 54.5 years were included. 53 pts. received TEM/CAP and 13 pts. TEM. TEMbased CTx was given in pancreatic (p) NEN $(n=47)$, bronchopulmonary (b) NEN ( $=9)$, NEN with unkown primary $(\mathrm{n}=5)$, intestinal NEN $(n=3)$ and other NEN $(n=2) .2$ pts. had NET-G1, 48 pts. had NET-G2 and 16 pts. had NEN-G3 (6 pts. NET-G3, 10 pts. NECG3). TEM-based CTx was 1st-line therapy in $10 \%$, 2nd line in $26 \%$, 3rd line in $25 \%$, 4th line in $23 \%$, 5th line in $9 \%$ and 6 th line in $7 \%$. Best radiologic response (RR) was $24 \%$, while disease control rate (DCR) was $69 \%$ for the whole cohort. In NET-G1/2 and NET/C-G3 RR was $15 \%$ and $58 \%$, while DCR was $68 \%$ and $75 \%$ respectively. Median overall survival since start of therapy (OS) was 43 months (m), while median time to progression (mTTP) was $6 \mathrm{~m}$. In p-NEN mTTP was $6 \mathrm{~m}$, while $5 \mathrm{~m}$ in b-NEN. In NET-G1/2 median TTP was $5 \mathrm{~m}, 8 \mathrm{~m}$ in NEC-G3 and $11 \mathrm{~m}$ in NET-G3. Conclusion: TEM-based therapy showed good DCR of NET-G1/G2 and NEN-G3, while RR was moderate. TTP in NET-G1/G2 was lower than reported in the literature due to later usage in Tx-line. Keywords: temozolomide, capecitabine.

\section{J10 \\ Retrospective Comparison of Chemotherapy Treatment with Capecitabine Alone or Fluropirimidine Plus Oxaliplatin in Advanced and Pretreated NET}

\author{
Marconcini R. ${ }^{a}$, Ricci S. ${ }^{\mathrm{b}}$, Galli L. ${ }^{\mathrm{a}}$, Antonuzzo A. ${ }^{\mathrm{b}}$,

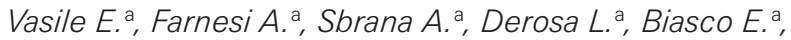 \\ Falcone $A{ }^{\text {a }}$ \\ aDivision of Medical Oncology 2, Azienda Ospedaliero- \\ Universitaria Pisana, IstitutoToscana Tumori, Pisa, Italy; \\ ${ }^{b}$ Division of Medical Oncology 1, Azienda Ospedaliero- \\ Universitaria Pisana, IstitutoToscana Tumori, Pisa, Italy
}

Introduction: Oxaliplatine plus fluoropirimidine and capecitabine treatment alone are considered investigational treatments in well-differentiated (WD) NET. Aim(s): We evaluate our experience in metastatic WD NET patients (pt) treated with Oxaliplatine based regimen (OxaliCT) and Capecitabine $(\mathrm{Cp})$. Materials and Methods: From October 2005 to September 2014, 56 pretreated WD NET pt with progressive disease were treated with $\mathrm{Cp}$ regimen (31 pt) or OxaliCT (25 pt) (2 pt exluded for toxicity after first cicle). $\mathrm{Pt}$ in $\mathrm{Cp}$ regimen received $\mathrm{Cp}$ only as chemotherapy, $\mathrm{pt}$ in OxaliCT regimen recived capecitabine/oxaliplatin (XELOX), or leucovorin/ fluorouracil/oxaliplatin (FOLFOX). The primary tumor site was pancreas, gastrointestinal tract, lung and unknown in 20,16, 9 and $12 \mathrm{pt}$ respectively. Treatment efficacy was evaluated by response rate and in terms of PFS. Results: In OxaliCT regimen, 8 (34\%) pt had a PR, 10 pt (43\%) showed SD, 5 (21\%) pt showed PD; G3-G4 toxicities were neurotoxicity and diarrhea. In Cp regimen, 1 (3\%) pt had a PR, 15 pt (48\%) showed SD, 15 (48\%) pt showed PD; G3-G4 toxicities were not reported. PFs for OxaliCT and $\mathrm{Cp}$ regimens were 8.6 and 5.57 months respectively (P 0.0629 HR 0.761 95\% CI 0.4305 to 1.346). Conclusion: $\mathrm{Cp}$ regimen and OxalCT regimen showed interesting activity and efficacy in pretreated pt with progressive WD NET, with no statistically different PFS even if clinical relevant. OxaliCT showed more PR and more G3 toxicities: these results could give a role to $\mathrm{Cp}$ regimen in selected pt. Keywords: capecitabine, oxaliplatine.

\section{J11 \\ Chemotherapy with Capecitabine Plus Temozolomide (CAP-TEM) in Patients with Advanced Neuroendocrine Neoplasms (NENs): An Italian Multicenter Retrospective Analysis}

Spada F., Antonuzzo L. ${ }^{\text {, }}$ Marconcini R. ${ }^{c}$, Gelsomino F. Fumagalli C. a, Messerini L. b, Di Costanzo F. ${ }^{\mathrm{b}}$, Ricci S. ${ }^{\mathrm{c}}$, Luppi G. ${ }^{\mathrm{a}}$, Radice D. ${ }^{\text {a }}$ Galdy S. ${ }^{\text {a }}$ Cella C. ${ }^{\text {a, Barberis M. }}$, Fazio N. ${ }^{a}$

${ }^{a}$ European Institute of Oncology, Milan, Italy; ${ }^{b} \mathrm{AOU}$ Careggi, Firenze, Italy; 'University Hospital, Pisa, Italy; dUniversity Hospital, Modena, Italy

Introduction: A combination of capecitabine (CAP) and temozolomide (TEM) has been successfully used as first-line treatment in pancreatic neuroendocrine neoplasms (pNENs). $\mathbf{A i m}(\mathbf{s})$ : This is an Italian multicenter retrospective analysis to evaluate activity and toxicity of CAP-TEM regimen in patients with advanced NENs and potential correlation with some biological factors. Materials and Methods: Clinical records of patients from 4 centers were reviewed. Objective response rate $(\mathrm{ORR}=\mathrm{PR}+\mathrm{SD})$, and toxicity were calculated. The MGMT methylation-status (MGMT-gene $>5 \%=$ responders) and TS-polymorphisms $(2 \mathrm{R} / 2 \mathrm{R}, 2 \mathrm{R} / 3 \mathrm{R}=$ responders $)$ in tumor tissue/peripheral-blood were evaluated by pyrosequencing. Results: 52 patients were consecutively treated. The primary tumor was: pancreas in 52\% patients, gastrointestinal in 13\%, unknown in $12 \%$, lung in $23 \%$. The vast majority were low/intermediate grade of malignancy and $12 \%$ were 'low G3' (Ki67 21-55\%). $83 \%$ patients were progressive on different therapies. ORR was evaluable in $73 \%$ patients. Partial-response (PR) occurred in 29\% patients (95\% CI: 17-44), stable-disease (SD) in 43\% (95\% CI: 29-58). 3 out of 6 'low G3' responded. Thrombocytopenia was the most frequent grade-3 toxicity, always temporary. Five out 15 PR (33\%) patients had genotype $2 \mathrm{R} / 3 \mathrm{R}$ or $2 \mathrm{R} / 2 \mathrm{R}$, and $46 \%$ MGMT-gene inactivation by epigenetic silencing. Conclusion: CAP-TEM therapy could be active and well tolerated in pre-treated patients with advanced NENs. A prospective study to validate the biologic methods would be justified. Keywords: nen, net, chemotherapy. 
$\mathrm{J} 12$

\section{Evaluation of the Combination Oxaliplatin and 5-Fluorouracil or Gemcitabine in Patients with Metastatic Lung Carcinoid Tumors}

\author{
Walter T. ${ }^{\mathrm{a}}$, Planchard D. ${ }^{\mathrm{b}}$, Bouledrak K. ${ }^{\mathrm{a}}$, Guigay J. ${ }^{\mathrm{b}}$, \\ Dussol A.S. ${ }^{a}$, Scoazec J.Y. ${ }^{\mathrm{b}}$, Hervieu V. ${ }^{\mathrm{a}}$, Ducreux M. ${ }^{\mathrm{b}}$, \\ Lombard-Bohas C. a, Baudin E. \\ aHospices Civils de Lyon, Hôpital Edouard Herriot, Lyon, \\ France; 'bustave Roussy, Villejuif, France
}

Introduction/Aim(s): The aim of this study was to retrospectively analyse the efficacy of gemox and folfox in the treatment of metastatic lung carcinoid tumors. Materials and Methods: 44 patients were included in two tertiary centres between January 1999 and January 2013 in this retrospective study. Typical, atypical carcinoids or not otherwise specified carcinoids were found in $20 \%, 54 \%$, and $26 \%$. Patients had synchronous $(41 \%)$ or metachronous $(59 \%)$ metastasis disease. $79 \%$ were progressive before start of chemotherapy. Treatment consisted of: gemcitabine $1000 \mathrm{mg} / \mathrm{m}^{2}$ and oxaliplatin $100 \mathrm{mg} / \mathrm{m}^{2}$ every 2 weeks (gemox regimen, $\mathrm{n}=21$ ) or 5 -fluorouracil and oxaliplatin $85 \mathrm{mg} / \mathrm{m}^{2}$ (folfox regimen, $\mathrm{n}=21$ ) every 2 weeks. Results: They received oxaliplatin-based chemotherapy in first-line (19\%), second-line (33\%), or post-second-line (47\%). The median number of cycles was 7 [1-12]. Seven $(17 \%)$ had a partial response, and $29(69 \%)$ had stable disease. Median progression free survival was 14 months and median overall survival was 35 months. No significant difference was observed in response, PFS, OS between both regimens. The two factors associated with better PFS after multivariate analysis were typical carcinoids according to WHO classification $(\mathrm{HR}=0.18(0.04-0.84), \mathrm{p}=0.03)$ and performance status $0-1(\mathrm{HR}=$ $0.12(0.02-0.61), p=0.01)$. Conclusion: Gemcitabine-oxaliplatin or 5-fluorouracil-oxaliplatin combination seems attractive in metastatic pulmonary carcinoid tumors. Keywords: gemox, folfox, lung, carcinoid.

\section{Medical Treatment - SMS Analogues, Interferon}

K1

\section{Population Pharmacokinetic (PK) Analysis of Lanreotide Autogel (LAN) in the Treatment of Patients (pts) with NETs: Pooled Analysis of Four Clinical Trials}

Buil-Bruna N. ${ }^{a}$, Jesús Garrido M. ${ }^{a}$, Dehez M. ${ }^{\mathrm{b}}$, Manon A. ${ }^{\mathrm{b}}$, Nguyen T.X.Q. ${ }^{\text {b }}$ Gomez-Panzani E. ${ }^{\text {b, Trocóniz I.F. }}{ }^{\mathrm{a}}$

aSchool of Pharmacy, University of Navarra, Pamplona, Spain; ' Ipsen, Les Ulis, France

Introduction: LAN provide antitumor effects and symptom control in NET pts. Aim(s): To characterize the PK of LAN 60, 90, or 120 mg SC injections every 4 wks in NET pts. Materials and Methods: 1541 serum concentrations were obtained from 290 pts and analyzed simultaneously using a population approach with NONMEM v7.2 software. The evaluated covariates included demographics, renal and hepatic function, and disease characteristics. Results: Serum profiles were described using a one-compartment disposition model and two parallel absorption pathways following first and zero order kinetics. Estimated apparent volume of distribution was $18.3 \mathrm{~L}$, and apparent total serum clearance for a typical $74 \mathrm{~kg}$ pt was $513 \mathrm{~L} /$ day. Most covariates had no statistically significant effects on PK. The only exception was a moderate effect of body weight on clearance $(+30 \%$ in weight resulted in $+25 \%$ in clearance); however, this was not clinically relevant as low - vs. high-weight pts (51 vs. $104 \mathrm{~kg}$ ) would still have overlapping PK profiles. The intra-pt variability (IPV) was low for clearance $(27 \%)$, moderate for absorption constant $(61 \%)$, but high for volume of distribution ( $150 \%$; due to a lack of PK timepoints around the Cmax). At steady state, mean \pm SD AUC was $239.0 \pm$ $64.8 \mathrm{ng}$.day $/ \mathrm{mL}$ and Cmax was $13.9 \pm 7.4 \mathrm{ng} / \mathrm{mL}$, showing moderate IPV. Conclusion: This analysis shows moderate IPV in PK of LAN and no clinically important factors impacting on PK in NET pts that would require a potential dose adjustment in clinical practice. Supported by Ipsen. Keywords: pk, dosing. 
K2

\section{Antitumor Treatment with Lanreotide Autogel $120 \mathrm{mg}$ (LAN) for Enteropancreatic (EP-)NET: Update from the CLARINET Open-Label Extension (OLE) Study}

\author{
Caplin M. ${ }^{\text {a }}$, Pavel M. ${ }^{\text {b }}$, Cwikla J.B. ${ }^{c}$, Phan A.T. ${ }^{\text {, }}$, \\ Raderer M. ${ }^{\text {e }}$ Sedláčková E. ${ }^{\dagger}$ Cadiot G. ${ }^{\mathrm{g}}$, Wolin E.M. ${ }^{\mathrm{h}}$, \\ Capdevila J.', Wall L.', Rindi G. ${ }^{k}$, Langley A.! \\ Gomez-Panzani E., Ruszniewski P.B. ${ }^{\mathrm{m}}$ \\ ${ }^{a}$ Royal Free Hospital, London, UK; ${ }^{b}$ Charité University \\ Medicine Berlin, Berlin, Germany; 'University of Varmia \\ and Masuria, Olsztyn, Poland; dThe Methodist Hospital, \\ Houston, Tex., USA; eUniversity Hospital, Vienna, Austria; \\ fFirst Faculty of Medicine and General Teaching Hospital, \\ Prague, Czech Republic; gRobert-Debré Hospital, Reims,

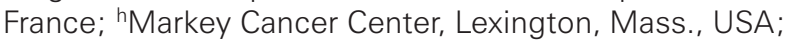 \\ 'Vall d'Hebron University Hospital and Vall d'Hebron \\ Institute of Oncology, Barcelona, Spain; Western General \\ Hospital, Edinburgh, UK; ' Università Cattolica del Sacro \\ Cuore, Rome, Italy; 'Ipsen, Les Ulis, France; MBeaujon \\ Hospital, Clichy, France
}

Introduction: In CLARINET, LAN had significant antitumour effects vs. placebo (PBO) on metastatic G1/G2 EP-NETs, liver burden $\leq$ and $>25 \%$, and $96 \%$ SD prior to LAN. Aim(s): To characterise LAN antitumour activity further in the OLE. Materials and Methods: Core study $(\mathrm{n}=204)$ : patients had metastatic well $/$ moderately differentiated non-functioning EP-NETs with Ki-67 $<10 \%$, no prior medical therapy within 6 mo, documented PD/SD status, and received LAN/PBO for 96 wks or until PD (RECIST 1.0) or death. OLE $(n=88)$ : patients entered the OLE if they had SD at end of core study or they had PD on PBO during core study; LAN is administered until (further) PD or death (NCT00842348). Results: Median PFS on LAN (time from randomisation to first PD in core or OLE) was 32.8 mo (midgut/pancreas: 42.8/29.7 mo; grade 1/2: 42.8/29.7 mo; HTL $\leq>>25 \%: 42.8 / 24.1 \mathrm{mo}$ ). Median time from first PD on PBO (core) to further PD on LAN (OLE): 14.0 mo (i.e. 2nd progression). During the OLE, $66 \%$ who continued LAN vs. $81 \%$ who switched to LAN had adverse events, most commonly diarrhoea. Safety profiles were similar in subgroups. No new safety concerns were identified in any population. Conclusion: Antitumour benefits continued in patients without PD on LAN for over 2 years and may also be achieved with LAN in patients having progressed on PBO. Supported by Ipsen. Keywords: lanreotide, antitumour effects.
K3

Health-Related Quality of Life (HRQoL) with Lanreotide Autogel (LAN) $120 \mathrm{mg}$ in Patients with Enteropancreatic (EP-)NETs: Post Hoc Analyses from the CLARINET Study

\author{
Caplin M. ${ }^{\text {a }}$, Pavel M. ${ }^{\mathrm{b}}$, Cwikla J.B. ${ }^{\mathrm{c}}$, Phan A.T. ${ }^{\mathrm{d}}$, \\ Raderer M. ${ }^{e}$, Sedláčková E. , Cadiot G. ${ }^{\mathrm{f}}$, Wolin E.M. ${ }^{\mathrm{h}}$, \\ Capdevila J.', Wall L.', Rindi G. ${ }^{\text {, }}$ Langley A. ', \\ Gomez-Panzani E. ', Ruszniewski P.B. ${ }^{\mathrm{m}}$ \\ ${ }^{a}$ Royal Free Hospital, London, UK; ${ }^{b}$ Charité University \\ Medicine Berlin, Berlin, Germany; 'University of Varmia \\ and Masuria, Olsztyn, Poland; 'The Methodist Hospital, \\ Houston, Tex., USA; eUniversity Hospital, Vienna, Austria; \\ ${ }^{f}$ First Faculty of Medicine and General Teaching Hospital, \\ Prague, Czech Republic; gRobert-Debré Hospital, Reims, \\ France; ' Markey Cancer Center, Lexington, Mass., USA; \\ 'Vall d'Hebron University Hospital and Vall d'Hebron \\ Institute of Oncology, Barcelona, Spain; Western General \\ Hospital, Edinburgh, UK; ' Università Cattolica del Sacro \\ Cuore, Rome, Italy; 'Ipsen, Les Ulis, France; mBeaujon \\ Hospital, Clichy, France
}

Introduction: In CLARINET, favourable efficacy and safety with LAN in metastatic EP-NETs was associated with stable HRQoL outcomes. Aim(s): To report exploratory post hoc analyses of treatment impact on specific aspects of HRQoL, and explore possible prognostic factors for changes in these HRQoL aspects. Materials and Methods: Patients $(\mathrm{n}=204)$ completed EORTC QLQ-C30 and QLQ-GI.NET21 every 12 wks. Analyses reported are: GI.NET21 endocrine (ENDO) and GI symptoms scales; QLQ-C30 physical functioning (PF) scale. Treatment effects assessed using logistic regression to determine relative odds of unchanged/improved vs. deteriorated HRQoL at last value within subgroups defined by baseline (BL) characteristics. Prognostic factors (BL characteristics) assessed for unchanged/improved vs. deteriorated HRQoL using exploratory logistic regression model $(\mathrm{p}<0.1$ for inclusion/exclusion). Results: Treatment effects - LAN vs. PBO for overall population: OR [95\% CI]: ENDO, 1.02 [0.55, 1.88]; GI, 0.93 [0.52, 1.65]; PF, $0.98[0.55,1.75]$; LAN vs. PBO changes generally similar across subgroups. Prognostic factors $(\mathrm{p}<0.05$ in final model) - patients with unchanged/improved HRQoL related to GI symptoms and PF more likely to have liver burden $\leq 25 \%$; other factors were lower BMI (for ENDO) and higher BL scores (for ENDO and GIHRQoL scores). Conclusion: LAN $120 \mathrm{mg}$ provided PFS benefits without adversely affecting HRQoL related to ENDO, GI symptoms or PF. Possible prognostic factors for HRQoL changes include BL liver burden. Supported by Ipsen. Keywords: lanreotide, quality of life. 
K4

\section{Chromogranin A (CgA) and PFS Outcomes in Lanreotide Autogel (LAN) in Patients with Metastatic Enteropancreatic (EP-) NETs: Data from the CLARINET Study}

\author{
Caplin M. ${ }^{\text {a }}$ Pavel M. ${ }^{\text {, }}$, Cwikla J.B. ${ }^{c}$, Phan A.T. ${ }^{\text {, }}$, \\ Raderer M. ', Sedláčková E. , Cadiot G. ${ }^{\mathrm{f}}$, Wolin E.M. ${ }^{\mathrm{h}}$, \\ Capdevila J.', Wall L.', Rindi G. ${ }^{\text {, }, ~ L a n g l e y ~ A . !, ~}$ \\ Gomez-Panzani E.', Ruszniewski P.B. ${ }^{\mathrm{m}}$

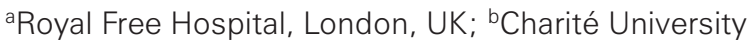 \\ Medicine Berlin, Berlin, Germany; 'University of Varmia \\ and Masuria, Olsztyn, Poland; 'The Methodist Hospital, \\ Houston, Tex., USA; eUniversity Hospital, Vienna, Austria; \\ ${ }^{f}$ First Faculty of Medicine and General Teaching Hospital, \\ Prague, Czech Republic; gRobert-Debré Hospital, Reims, \\ France; ' Markey Cancer Center, Lexington, Mass., USA; \\ 'Vall d'Hebron University Hospital and Vall d'Hebron \\ Institute of Oncology, Barcelona, Spain; Western General \\ Hospital, Edinburgh, UK; kUniversità Cattolica del Sacro \\ Cuore, Rome, Italy; 'Ipsen, Les Ulis, France; mBeaujon \\ Hospital, Clichy, France
}

Introduction: The CLARINET study showed LAN $120 \mathrm{mg}$ prolonged PFS vs. placebo (PBO) in patients with metastatic EP-NETs. $\operatorname{Aim}(\mathbf{s})$ : To explore the relationship between CgA and PFS outcomes. Materials and Methods: Tumour progression (CT scan) and plasma $\mathrm{CgA}$ were assessed regularly over 96 wks. PFS outcomes (RECIST 1.0) and tumour response (SD/PD) were evaluated according to change in $\mathrm{CgA}$ from baseline to wk 12 and last value (LV) in patients with baseline levels above normal. Results: A greater \% of LAN than PBO patients with elevated baseline $\mathrm{CgA}(\mathrm{n}=65$ and $\mathrm{n}=$ 64 respectively) had $\geq 30 \%$ reductions at $12 \mathrm{wks}$ ( 74 vs. $15 \%)$ and at LV (59 vs. $11 \%)$. More LAN patients with SD $(n=33)$ than with PD $(\mathrm{n}=18) \mathrm{had} \geq 30 \% \mathrm{CgA}$ reductions at $12 \mathrm{wks}(81 \mathrm{vs} .65 \%)$ and at $\mathrm{LV}$ (70 vs. $44 \%)$. In PBO patients, similar numbers with SD $(\mathrm{n}=11)$ or with $\mathrm{PD}(\mathrm{n}=39)$ showed reductions at $12 \mathrm{wks}(27 \mathrm{vs.} 17 \%)$ and at LV (18 vs. $10 \%$ ). Trends were similar for those with $\geq 50 \%$ reductions. There was a consistent treatment effect with LAN vs. PBO on PFS over the 96 weeks in patients with or without a $\geq 30 \% \mathrm{CgA}$ reduction (HR [95\% CI]: $0.44[0.19,1.04]$ vs. $0.63[0.26,1.52])$. Conclusion: This exploratory analysis suggests $\mathrm{CgA}$ response may be associated with progression of EP-NETs. Supported by Ipsen. Keywords: lanreotide, biomarker.

\section{K5}

\section{IFNa versus IFNa Plus Octreotide LAR in Treatment of NETs}

\section{Gorbunova V., Emelianova G., Orel G., Markovich A.,}

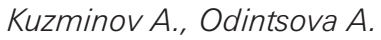

N.N.Blokhin Russian Cancer Research Center, Moscow, Russia

Introduction: It is still unknown whether the combination of IFN $\alpha$ with octreotide LAR has benefit compared with IFN $\alpha$ alone. $\operatorname{Aim}(\mathbf{s})$ : To compare efficacy and toxicyty of IFN $\alpha$ and IFN $\alpha$ plus Octreotide LAR in first line of therapy of patients with NETs. Materials and Methods: 50 pts with morphologically verified well differentiated advanced NETs (G1-2), 16 (32\%) men and 34 (68\%) women, mean age $55(19-80)$. IFN $\alpha(\mathrm{n}=19)$ : lung-11 (58\%); GEP-8 (42\%). Grade: G1-8 (42\%); G2-11 (58\%). IFN $\alpha+$ Octreotide LAR $(\mathrm{n}=31)$ : lung-6 (19\%); GEP-25 (81\%). Grade: G1-11 (35\%); G2-20

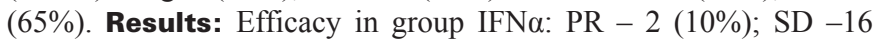
$(85 \%)$, PD $-1(5 \%)$. Efficacy in group IFNa+Octreotide LAR: PR 2 (6\%); SD - 26 (84\%); PD - 3 (10\%). Median TTP (MTTP) - 35 and 24 mo $(\mathrm{p}=0.11)$, respectively. MTTP in patients with lung NETs and GEP NETs was 35 and 23 mo $(\mathrm{p}=0.2)$, respectively. Toxicity did not differ. Of AEs: diarrhea II -2 (4\%), fever I-II-6 (12\%), thrombocytopenia I-III-3 (6\%), weakness I-II - $8(16 \%)$. Conclusion: There was trend to longer TTP in lung NETs pts comparing with GEP NETs pts treated with IFN $\alpha$. IFN $\alpha$ may be a therapy of choice in pts with well differentiated lung NETs as first line treatment. IFN $\alpha$ have a similar toxicity profile comparing with IFN $\alpha$ combined with Octreotide LAR. Further study needed. Keywords: ifn $\alpha$, octreotide lar, nets.

\section{K6}

\section{Somatostatin Analogues in Bronchial Neuroendocrine Tumors: Symptom Control and Anti-Proliferative Role}

Karra E. ${ }^{a}$, Polycarpou A. a , Tanaskovic N. ${ }^{\text {a, }}$ Garcia-Hernandez J. ${ }^{a}$, Mullan M. ${ }^{\text {, }}$ Caplin M. ${ }^{\text {, }}$ Toumpanakis $C$.

aENETS Centre Royal Free Hospital, London, UK; bENETS Centre Royal Free Hospital, UK

Introduction: There is no established fist-line medical treatment for advanced bronchial NETs. Aim(s): To assess the symptom-control \& antiproliferative efficacy (time-to-first-radiological-progression) of somatostatin-analogues (SA). Materials and Methods: Patients with histologically confirmed bronchial-NETs (typical/ atypical-carcinoids), with \& without primary resection, and with tumour-uptake in Octreoscan \&/or Ga-68-Octreotate PET were retrospectively reviewed. None had received any systemic treatment prior to SA-commencement. A symptom-score \& radiological assessment by RECIST were utilized. Results: 22-patients (age: mean = $59.1 \pm 2.9$ years, range $=33-75$ years $) ; 13$-male $/ 9$-female were included. $54.5 \%$ had undergone primary resection. $90.5 \%$ were typical and $9.5 \%$ atypical carcinoids. In $71.4 \% \mathrm{Ki} 67$ was $<2 \%$ and $28.6 \%$ 
between 2-20\%. Mean-time of SA-initiation, post-disease diagnosis, was 46.4 months. SA were commenced exclusively for symptomcontrol (carcinoid syndrome and/or respiratory symptoms) in $18 \%$, tumor-growth control in $41 \%$ and for dual purpose in $41 \%$ of patients. Symptom control was achieved in $95.5 \%$. Radiological progression (progressive-disease/PD), post-SA introduction, occurred in $45.5 \%$, whilst in $54.5 \%$ the disease remained stable (SD). In PD cases, mean-time-to radiological-progression was 16.5 months. Tumour grade negatively correlated with time-to-progression $(\mathrm{r}=-0.428, \mathrm{P}=$ $0.053)$. Conclusion: SA can delay disease progression and induce substantial symptomatic relief, mainly in typical bronchial carcinoids. Keywords: bronchial net.

\section{K7}

\section{High-Dose Treatment with Somatostatin Analogs in Neuroendocrine Tumors}

Modica R. ${ }^{a}$, Ramundo V. ${ }^{\text {, Marciello F. }}$, Marotta V. , Pizza G. a, Carratù A.C. a, De Luca di Roseto C. , Buonomano P. , Giordano C. b, Trimarchi F.', Colao A. a, Faggiano $A .^{\text {a }}$

a'Federico II' University, Naples, Italy; ' University of Palermo, Palermo, Italy; 'University of Messina, Messina, Italy

Introduction: Somatostatin analogs (SSA) effectively control symptoms in neuroendocrine tumours (NET), besides showing antiproliferative activity. In progressive or metastatic NET, increasing SSA dose or shortening the dosing interval is common clinical practice, though empirical. Aim(s): Aim of this study is to evaluate efficacy and safety of high-dose SSA treatment in pts with progressive disease under standard SSA dose. Materials and Methods: Twenty-one pts (median age 56.8 yrs) with NET of different origin were retrospectively identified among 118 pts under SSA therapy. All 21 pts were treated with SSA high dose schedule treatment, after disease progression under standard dose. The median follow-up was 22.3 months (range 4-76). High dose schedule included octreotide LAR in 15 pts (73\%) and lanreotide Autogel in 6 (27\%). Results: Progression free survival was significantly higher with high-dose treatment compared with standard dose (32 vs. 8 months, $\mathrm{p}<0.05$ ). Partial objective tumor response was recorded in 1 patient $(5 \%)$, stabilization in $10(47.5 \%)$ and progression in $10(47.5 \%)$. Among 16 pts who were symptomatic under standard dose, complete clinical response was obtained in $1(6 \%)$, partial response in $9(57 \%)$. Side effects were abdominal discomfort (5\%), asymptomatic gallstones (5\%) and type 2 diabetes mellitus (5\%). Conclusion: High-dose SSA treatment in progressive NET is still effective in pts refractory to standard SSA doses. No additional toxicity is observed compared with standard dose. Keywords: somatostatin analogs, neuroendocrine tumors.

\section{K8 \\ Efficacy of Lanreotide versus \\ Follow-Up in Early-Stage \\ Duodeno-Pancreatic Neuroendocrine Tumors (NETs) Related to Multiple Endocrine Neoplasia Type 1 (MEN1): Preliminary Data}

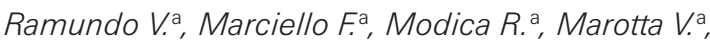 \\ Pizza G. ${ }^{a}$, Camera L. ${ }^{a}$, Napolitano V. ${ }^{\text {, }}$ De Luca L. ${ }^{\text {, }}$ \\ Colao A. ${ }^{\text {a }}$ Faggiano A. ${ }^{a}$ \\ aFederico II University, Naples, Italy; ${ }^{b}$ Second University of \\ Naples, Naples, Italy; ' Pellegrini Hospital, Naples, Italy
}

Introduction: Surgery is the only curative approach for NETs, representing the first-line therapy. As most pts with MEN1 have multiple duodeno-pancreatic NETs, cure is generally not possible, unless to adopt radical surgery. Somatostatin analogues (SSAs) represent one of the main therapeutic options in functioning well-differentiated NETs. There are no perspective studies focusing on MEN1-related NETs. Aim(s): To evaluate the effectiveness of lanreotide in pts with early-stage MEN1-related duodeno-pancreatic NETs. Materials and Methods: Since February 2012, all MEN1 pts with US-endoscopy and/or contrast-enhanced CT/MRI evidence of NETs within duodenum and pancreas $(\leq 10 \mathrm{~mm}$ of maximal diameter) were enrolled. Eighteen pts ( $9 \mathrm{M}$ and $9 \mathrm{~F}, 20-62 \mathrm{yrs}$ ) with NETs of $5.7 \mathrm{~mm}$ as average (range, $3-10 \mathrm{~mm}$ ) were randomized in 2 groups: group A receiving lanreotide autogel $120 \mathrm{mg} / 28$ days, group B without any treatment. All pts were evaluated with US-endoscopy plus CT/MRI every 6 months. At now, there are 6 pts in group A and 12 in group B. Mean follow-up time is 13.4 months (range 6-24). Results: In group A, 2 pts had minor response and 4 stable disease. In group B, 11 pts had stable disease and 1 had tumor progression after 16 months and started therapy with lanreotide $120 \mathrm{mg} / 28$ days. Conclusion: These preliminary data suggest that lanreotide could be effective as antiproliferative agent in pts with early-stage MEN1-related duodenopancreatic NETs. Keywords: multiple endocrine neoplasia type 1, lanreotide, duodeno-pancreatic nets. 
K9

\section{Treatment Satisfaction, Symptom Control and Quality of Life (QoL) with Lanreotide Autogel (LAN) in NET Patients with Carcinoid Syndrome (CS): Results from the SYMNET Study}

Ruszniewski Pa , Caplin M. ${ }^{\text {b }}$, Valle J. ${ }^{c}$, Lombard-Bohas C. ${ }^{d}$ Poston G. ${ }^{\mathrm{e}}$, Perros P. , Holubec L. ${ }^{\mathrm{f}}$, Delle Fave G. , Smith D. ${ }^{\mathrm{i}}$, Niccoli P. ${ }^{\mathrm{j}}$, Maisonobe P. , Atlan P.

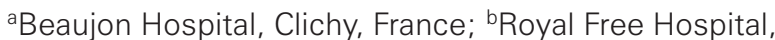
London, UK; ' ${ }^{c}$ Christie NHS Foundation Trust, Manchester, UK; dEdouard-Herriot Hospital, Lyon, France; eUniversity Hospital Aintree, Liverpool, UK; 'Freeman Hospital, Newcastle-upon-Tyne, UK; ${ }^{9}$ Teaching Hospital Plzen, Plzen-Bory, Czech Republic; ' Ospedale Sant'Andrea, Rome, Italy; iSaint André Hospital, Bordeaux, France; ' $\mathrm{CHU}$ Timone, Marseille, France; ${ }^{k}$ Ipsen, Boulogne-Billancourt, France

Introduction: CS associated with NETs can have a negative impact on patients' QoL. Aim(s): To evaluate QoL data from the SYMNET study (NCT01234168). Materials and Methods: Primary endpoint was patient satisfaction with diarrhoea control. Symptoms assessed from medical records. QoL was assessed using the EORTC QLQ-C30 and QLQ-GI.NET21 questionnaires, which assess functional dimensions (QLQ C30) and symptoms (QLQC30 and QLQ-GI.NET21; lower scores = better QoL). Overall QoL was evaluated according to satisfaction with CS symptom control. Results: Of 273 patients enrolled, $76 \%(95 \%$ CI: 70, 81) were 'completely' or 'rather' satisfied with diarrhoea control. There was a clinically significant reduction in stool number and statistically significant improvements in urgency, leakage and associated pain since LAN initiation. Scores for QLQ-C30 global health status were high (mean [SD] 65.5 [22.1], median [range] 66.7 [0-100]); scores for QLQ-GI.NET21 endocrine and gastrointestinal (GI) subscales were low (endocrine, mean [SD] 19.4 [21.3], median [range] 11.1 [0-100]; GI, mean [SD] 22.9 [17.8], median [range] 20.0 [0-87]). In total, 70\% of patients 'rather' or 'completely satisfied' had 'good', 'very good' or 'excellent' QoL. Conclusion: In a real-world setting, CS symptom control with LAN treatment translates into favourable levels of satisfaction among NET patients. Higher satisfaction levels were consistent with higher global health QoL scores. Supported by Ipsen. Keywords: quality of life.
K10

\section{Predictive Factors for Antiproliferative Activity of Octreotide LAR in Advanced Neuroendocrine Tumors}

Toumpanakis C. a Laskaratos Fa', Maragkoudakis E. ${ }^{a}$, Naik K. ${ }^{a}$, Oikonomopoulos N. ${ }^{a}$, Walker M. ${ }^{b}$, Grant L. ${ }^{\text {c, }}$ Meyer T. ${ }^{\mathrm{d}}$, Caplin M. ${ }^{\text {a }}$

${ }^{a}$ Neuroendocrine Tumour Unit, Centre for

Gastroenterology, Royal Free Hospital NHS Foundation

Trust, London, UK; ' ${ }^{\prime}$ mperial College London, London,

UK; ' Radiology Department, Royal Free Hospital NHS

Foundation Trust, London, UK; dUniversity College London

Cancer Institute, London, UK

Introduction: The antiproliferative activity of Octreotide LAR in Neuroendocrine Tumors (NETs) has been demonstrated by small retrospective studies and confirmed by a prospective phase III trial (PROMID). However, there are limited data about the duration and predictors of response. $\mathbf{A i m}(\mathbf{s})$ : The aim of our retrospective study was to determine the time to radiological progression (TTRP) of disease and the factors that were associated with better response. Materials and Methods: 254 patients with advanced NETs and positive somatostatin receptor scintigraphy were included. Radiological assessment was based on RECIST criteria. Univariate and multivariate analyses were used to identify predictive factors. Median TTRP and 95\% confidence intervals were calculated using Kaplan-Meier analysis. Results: The median TTRP was 37 months (95\% CI, 32-52 months). There was a statistically significant shorter TTRP in patients with pancreatic primary (p: 0.001), G2 tumors (p: 0.001), high hepatic tumor volume (p: 0.006), and baseline Chromogranin- $\mathrm{A}(\mathrm{CgA})$ levels $>10$ times the upper normal limit (p: 0.006). Mean time to progression was longer in patients with stable disease at presentation (53 months). Age, mesenteric mestastases, desmoplasia and previous resection of primary did not affect progression. Female sex and skeletal metastases had some negative, but no statistically significant, effect. Conclusion: Small bowel primary, G1 tumors, low hepatic tumor volume, lower $\mathrm{CgA}$ levels and stable disease at presentation are favourable predictive factors of response. Keywords: octreotide lar, nets.

\section{K11}

\section{Somatostatin Responsive ACTH and Precursor Excess in a Midgut Mesentery NET}

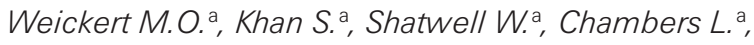

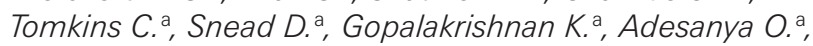
Dimitriadis G.K. ${ }^{a}$, Darby C. ${ }^{a}$, Randeva H.S. ${ }^{\text {, }}$, White A. ${ }^{\text {, }}$, Narasimha $M^{\text {a }}$

aUniversity Hospitals Coventry \& Warwickshire NHS Trust, Coventry, UK; bUniversity of Manchester, Manchester, UK

Introduction: ACTH production from a midgut mesentery NET is extremely rare (1). A 62 y old woman presented with hypokalaemia $(2 \mathrm{mmol} / \mathrm{L})$ and hyperpigmentation, $11 \mathrm{y}$ after surgery of a pT3N1Mx non-functional G1 NET with SRS positive, non-resectable but sta- 
ble, residual mass encasing mesenteric vessels. Serum cortisol (3261 $\mathrm{nmol} / \mathrm{L}), \mathrm{ACTH}(796 \mathrm{ng} / \mathrm{L}), \mathrm{CgA}(530 \mathrm{pmol} / \mathrm{L})$ and urine total cortisol metabolites $(33920 \mu \mathrm{g} / 24 \mathrm{~h})$ including 14 sub-products indicated change of biological behaviour into a functioning NET. Pituitary or pulmonary sources were excluded. Aim(s): To test somatostatin (SS) responsiveness of ACTH excess. Materials and Methods: A test dose of SS $100 \mu \mathrm{g}$ sc was applied. Results: SS resulted in rapid (300 min) improvement of ACTH (222.9 ng/L), cortisol $(1700 \mathrm{nmol} / \mathrm{l})$, urine cortisol metabolites $(620 \mathrm{~min} ; 4620 \mu \mathrm{g} / 24 \mathrm{~h})$ and $\mathrm{K}+(3.2 \mathrm{mmol} / \mathrm{L} ; 48 \mathrm{~h}$ ); followed by resistance after 3 wk (cortisol $6362 \mathrm{nmol} / \mathrm{L}$, ACTH $617 \mathrm{ng} / \mathrm{L}$; pro-ACTH and POMC 140; ULN $<40 \mathrm{pmol} / \mathrm{L}$ ), despite SS dose intensification and adding ketoconazole/metyrapone for cortisol excess; resulting in a L3 fracture, reduced mobility and subsequent fatal pneumonia. Conclusion: Considering the $1-10 \%$ cross-reaction of ACTH precursors in ACTH assays (2), precursors were not sufficiently elevated to account for the observed ACTH excess, suggesting production of ACTH precursors by the NET and processing them to ACTH. Treatment with somatostatin was efficient in this context, at least in the short term. (1) Fasshauer et al. BMC Cancer 2006 (2) Stovold et al Br J Cancer 2013. Keywords: net, acth precursors, pomc, somatostatin.

\section{Medical Treatment - Targeted Therapies}

\section{L1 \\ Experience with Bevacizumab in Neuroendocrine Tumors}

\author{
Apostolidis L., Jäger D., Winkler E.C. \\ National Center for Tumor Diseases (NCT), Heidelberg, \\ Germany
}

Introduction: Bevacizumab has shown promising activity in neuroendocrine tumors (NET) in combination with a multitude of other substances. Aim(s): To analyze the efficacy and toxicity of bevacizumab-containing protocols in NET. Materials and Methods: We performed a retrospective analysis of all patients treated with bevacizumab-containing protocols for metastatic NET who presented at our center between August 2008 and December 2014. Results: A total of 18 patients with different primaries and grading could be identified. Bevacizumab was combined with somatostatin analogues in $55.6 \%$ of cases and with chemotherapy in $38.9 \%$. As best response, 2 patients showed a partial remission, 5 a minor response and 7 a stable disease, resulting in a disease control rate of $77.8 \%$. Primary progressive disease was documented in 3 patients $(16.7 \%)$, 1 patient $(5.6 \%)$ was lost to follow-up. Median progression free (PFS) and overall survival (OS) after start of bevacizumab was 9.8 and 16.9 months respectively. Main toxicity was arterial hypertension in $22.2 \%$ of patients. Conclusion: Bevacizumab-containing protocols show promising antitumor activity and a well manageable mild toxicity profile in NET. Keywords: neuroendocrine tumor, targeted therapy, bevacizumab, anti-angiogenesis.

\section{L2 \\ Results of Sunitinib Treatment of Advanced Neuroendocrine Neoplasms: 'Real-Life' Outcome Data from the German NET-Register}

\author{
Bacher M. ${ }^{a}$, Maasberg S. ${ }^{\text {, }}$ Fottner C. b, Begum N. , \\ Quietzsch D. ${ }^{\mathrm{d}}$, Skrobek-Engel G. ${ }^{a}$, Pavel M. ${ }^{a}$, Rinke A. ${ }^{\mathrm{e}}$, \\ Lahner H. ${ }^{\dagger}$, Pape U.F. ${ }^{\mathrm{a}}$ \\ aMedical Department of Gastroenterology and \\ Hepatology, University Medicine Charité, Berlin, Germany; \\ ${ }^{b}$ Department of Endcrinology and Metabolism, University \\ Medicine, Mainz, Germany; 'Department of Surgery, \\ University Hospital Schleswig-Holstein, Campus Lübeck, \\ Lübeck, Germany; dDepartment of Internal Medicine, \\ Clinical Centre of Chemnitz, Chemnitz, Germany; \\ eDepartment of Gastroenterology and Endocrinology, \\ University Hospital Gießen/Marburg, Campus Marburg, \\ Marburg, Germany; fDepartment of Endocrinology and \\ Metabolism, University Hospital, Essen, Germany
}

Introduction: Among modern treatments (Tx) for neuroendocrine neoplasms (NEN) sunitinib (SUN) has impacted Tx of primarily pancreatic NEN (pNEN). Aim(s): This study provides recent data on routine SUN-Tx from 5 centres of the German NET-Register. Materials and Methods: Multicentric, retrospective analysis of pts. with histologically proven NEN, progression prior to Tx initiation with SUN and with initial diagnosis from 1988-2013; statistical analysis used SPSS 19.0. Results: 36 pts. with a median age of 56 years at initial diagnosis were analyzed. Primaries were pancreatic (pNEN, $33 / 92 \%$ ), intestinal (iNEN 1/3\%), or unknown (2/5\%). Previous Tx included surgery, chemotherapy, somatostatin analogues, PRRT and ablative Tx. SUN was initiated as 1 st-line Tx in 5\%, 2nd-line in $8 \%$, 3rd-line in $20 \%$ and even later Tx in 67\%. Radiomorphologic best response (BR) was PR in $23 \%$, SD in $60 \%$ and PD $17 \%$; no CR was observed. BR in 27 pNEN was PR in $26 \%$, SD in 56\% and PD in $18 \%$. BR according to Ki67-grading was SD in one NET-G1, PR in $25 \%$, SD in $58 \%$ and PD in $17 \%$ in 24 NET-G2 and PR in $25 \%$, SD in $50 \%$ and PD in $25 \%$ in 4 NEN-G3. SUN-Tx was terminated due to adverse events in $14 \%$. Median overall survival was 44 months; median time-to progression (TTP) was 8 months with SUN-Tx for all, 8.5 months for pNEN and 8 months for NET-G2. Conclusion: These data from 5 German centres demonstrate acceptable response and tumor growth control rates with SUN given the fact that it was mostly used in pts. at a later Tx-line than during the approval clinical trial. Keywords: sunitinib, outcome. 
L3

\section{Clinico-Biological and Histological Predictive Factors and Markers of Response to Everolimus in Neuroendocrine Tumors}

\author{
Benslama N. ${ }^{a}$, Bollard J.b , Vercherat C. ${ }^{b}$, Roche C. ${ }^{b}$, \\ Hervieu V. ${ }^{c}$, Lombard-Bohas C. ${ }^{a}$, Scoazec J.Y. ${ }^{c}$, Walter T. ${ }^{a}$ \\ aHospices Civils de Lyon, Hôpital Edouard Herriot, \\ Service d'Oncologie Digestive, Lyon, France; ' INSERM, \\ UMR 1052, Lyon Cancer Research Center, Lyon, France; \\ "Hospices Civils de Lyon, Hôpital Edouard Herriot, Service \\ Central d'Anatomie et Cytologie Pathologiques, Lyon, \\ France
}

Introduction: Several systemic treatments are available for metastatic neuroendocrine Tumors (NETs), including everolimus, but there is no predictive factor of response to these treatments. $\operatorname{Aim}(\mathbf{s})$ : Our aim was to identify clinico-biological and histological markers of response to everolimus. Materials and Methods: We retrospectively reviewed 53 patients with NETs treated by everolimus. Markers of mTOR pathway (p-p70S6K) and angiogenesis (endoglin, CD34) were studied by immunohistochemistry. Prognostic factors of PFS were studied by Kaplan Meier analysis. Good responders (PFS $>12$ months) were compared to poor responders (PFS $<6$ months). Results: All 53 patients had progressive and metastatic disease, 40 had a pancreatic NET. A functional tumor $(\mathrm{HR}=0.32, \mathrm{p}=0.01)$, no bone metastasis $(\mathrm{HR}=8.07, \mathrm{p}=0.0008)$, chromogranin $\mathrm{A}<5 \mathrm{~N}$ before treatment $(\mathrm{HR}=3.42, \mathrm{p}=0.01)$, occurrence of high cholesterol $(\mathrm{HR}=$ $0.06, \mathrm{p}<0.0001)$ and low tumor expression of $\mathrm{p}-\mathrm{p} 70 \mathrm{~S} 6 \mathrm{~K}(\mathrm{HR}=2.51$, $\mathrm{p}=0.02)$ were associated with better PFS in multivariate analysis. A single metastatic site $(p=0.02)$, no bone metastasis $(p=0.03)$, occurrence of high cholesterol $(\mathrm{p}=0.003)$ and grade 3 lymphopenia $(\mathrm{p}=0.03)$, low expression of $\mathrm{p}-\mathrm{p} 70 \mathrm{~S} 6 \mathrm{~K}(\mathrm{p}=0.04)$ and endoglin $(\mathrm{p}=$ 0.05 ) were more frequent in good responders. Conclusion: Classic markers are not useful to predict response to everolimus. Occurrence of hypercholesterolemia and lymphopenia may be early markers of response, p-p70S6K and endoglin immunostaining may be prognosis or predictive markers of response. Keywords: neuroendocrine tumors, everolimus, markers, p-p70s6k.
L4

Pancreatic Location, High Proliferation and Low p-mTOR Expression Levels Are More Frequent in Neuroendocrine Tumor Patients Responsive to mTOR Inhibitors: Results from a Preliminary Study

Birocco N. ${ }^{a}$, Brizzi M.P. , Airoldi M. ' , De Angelis C. ${ }^{\text {, }}$ Ciuffreda L. ${ }^{a}$, Maletta F. ${ }^{\mathrm{e}}$, Piovesan A. ${ }^{\dagger}$, Rapa I. ${ }^{\mathrm{g}}$, Sapino A. ${ }^{\mathrm{e}}$, Scaldaferri M. h, Scagliotti G. ${ }^{\mathrm{g}}$, Papotti M. ${ }^{\mathrm{g}}$, Volante M. ${ }^{\mathrm{g}}$

aMedical Oncology 1, Città della Salute e della Scienza, Turin, Italy; ' Medical Oncology, San Luigi Hospital, Orbassano, Turin, Italy; 'Medical Oncology 2, Città della Salute e della Scienza, Turin, Italy; ' Gastroenterology, Città della Salute e della Scienza, Turin, Italy; ' Department of Medical Sciences, University of Turin, Turin, Italy; ¡Oncological Endocrinology, Città della Salute e della Scienza, Turin, Italy; 9Department of Oncology, University of Turin, Orbassano, Turin, Italy; hPharmacy Unit, Città della Salute e della Scienza, Turin, Italy

Introduction: MTOR inhibitors are approved for the treatment of advanced neuroendocrine tumors. However, scarce data are available on clinical or pathological predictors of response to these agents. $\operatorname{Aim}(\mathbf{s})$ : To test in a pilot series the presence of clinical and pathological predictors of response to mTOR inhibitors. Materials and Methods: Clinical and pathological characteristics (sex, age, location of the primary tumor, tumor grade and Ki-67 index) and the expression of phosphorylated forms of mTOR and p70S6K were correlated with response to therapy in 20 neuroendocrine tumor patients treated with everolimus. Results: Seven patients had partial response (PR), 10 were stable (SD) and three progressed (PD) under everolimus treatment. Pancreatic location and higher mean Ki-67 index were significantly associated with a better response $(\mathrm{p}=0.03$ and $\mathrm{p}=$ 0.04 , respectively). Phospho-mTOR and p-S6K levels were significantly correlated each other $(\mathrm{p}<0.0001)$. No differences in $\mathrm{p}-\mathrm{mTOR}$ and p-p70S6K expression levels were observed in tumors segregated according to tumor site. In paired matched samples, the expression of p-mTOR was increased in 5 out of 9 metastatic tissues, compared to the corresponding primary tumors. p-mTOR and p-p70S6K were not significantly correlated with response to treatment. However, all PD and a minority of PR/SD cases had high expression levels of p-mTOR. Conclusion: Some specific tumor characteristics seem to be associated with response to mTOR inhibitors and should be validated in larger series. Keywords: mtor inhibitor, biomarker. 


\section{L5}

\section{A Phase II Study of Axitinib in Advanced Carcinoid Tumors: Preliminary Results}

Cives M. ${ }^{a}$, Strosberg J. ${ }^{a}$, Campos T. ${ }^{\mathrm{a}}$, Weber T. ${ }^{\mathrm{b}}$, Nickerson M. ${ }^{\mathrm{b}}$, Atreya C. ${ }^{\mathrm{b}}$, Venook A. ${ }^{\mathrm{b}}$, Kelley R. ${ }^{\mathrm{b}}$, Valone T. ${ }^{\mathrm{a}}$, Coppola D. ${ }^{\mathrm{a}}$, Bergsland E. ${ }^{\mathrm{b}}$

${ }^{a} \mathrm{H}$. Lee Moffitt Cancer Center and Research Institute,

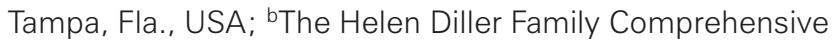
Cancer Center, University of California, San Francisco, Calif., USA

Introduction: NETs are highly vascularized neoplasms overexpressing VEGF as well as VEGFR. Axitinib is a TKI with selective picomolar potency against VEGFR-1, -2 and -3. Aim(s): To evaluate the safety and efficacy of axitinib in patients with advanced carcinoid tumors. Materials and Methods: We performed a phase II trial of axitinib $5 \mathrm{mg}$ BID in patients with advanced low to intermediate grade carcinoid tumors. Prior antiangiogenic therapy with a dedicated VEGF pathway inhibitor was not permitted. The primary endpoints were PFS and 1-year PFS rate. Results: 30 patients were enrolled and assessable for toxicity; 22 were assessable for response. Primary sites included small intestine (19), lung (3), unknown (3), colon (2), rectum (2), and thymus (1). 21 patients had low-grade and 9 patients had intermediate-grade tumors. Median TTF was 8.99 months (SD \pm 7.18 ) and the 12 -month PFS rate was $65 \%(\mathrm{SD} \pm 13 \%)$. The 1 -year OS rate was $93 \%$ ( $\mathrm{SD} \pm 4.9 \%$ ). Median PFS not yet determined due to small number of events. Best radiographic response was PR in $1 / 30(3.3 \%)$ and SD in $21 / 30(70 \%)$. Axitinib treatment was associated with a $90 \%$ rate of hypertension. Grade 3 and 4 hypertension were seen in $18(60 \%)$ and $2(7 \%)$ patients respectively and led to treatment discontinuation in two cases. However, axitinib interruption prompted a fast recovery without sequelae. Conclusion: The 12 mo PFS rate associated with Axitinib in advanced carcinoid tumors is promising. Although rates of hypertension were high, axitinib was overall well tolerated. Keywords: axitinib, anti-angiogenesis, vegfr.

L6

Gly388Arg FGFR4 Polymorphysm Is Not Predictive of Everolimus Efficacy in Gastrointestinal Well Differentiated Neuroendocrine Tumors (NET)

Cros J. ${ }^{a}$, Moati E. ${ }^{\text {, }}$ Raffenne J. ${ }^{a}$, Hentic O. ${ }^{b}$, Surcek M. ${ }^{c}$, Sbidian E. ', Bedossa P. , Paradis V.a, Sauvanet A. ',

Ruszniewski Pa, Couvelard A. ${ }^{\text {e }}$ Hammel Pa

aBeaujon Hospital-U1149 Paris Diderot University, Clichy, France; 'beaujon Hopital, Clichy, France; 'Saint Antoine Hospital, Paris, France; dHenri Mondor Hospital, Creteil, France; 'Bichat Hospital-U1149 Paris Diderot University, Paris, France

Introduction: Preclinical data suggest that single nucleotide polymorphism substituting an arginine $(R)$ for glycine $(G)$ in codon 388 of the FGFR4 transmembrane domain may increase proliferation of xenografted neuroendocrine cell lines and decrease their sensitivity to everolimus by modulating STAT3 signaling and the mTOR pathway. Aim(s): To evaluate the prognostic and predictive values of this polymorphism on everolimus efficacy in patients treated for a digestive NET. Materials and Methods: This monocentric retrospective cohort included patients with small bowel NET (SBNET) and pancreatic NET (PNET) treated by everolimus (2006-2013). Patients were genotyped by classical sequencing and mTOR pathway activity assessed by immunochemistry on FFPE samples (PTEN/ pPTEN/pAKT/pmTOR/pS6/p4EBP1). Results: Forty-one patients (21 males, median age $57 \mathrm{y})$ with a PNET $(\mathrm{n}=28)$ or a SBNET $(\mathrm{n}=$ $12)$, grade $1(n=6)$ or $2(n=37)$, were studied. At least one 388Arg allele was found in 14/23 PNET and 10/11SBNET. Progression free survival in the whole population and the PNET subgroup were not modified by the presence of one or two 388Arg alleles [HR $=1.31$ $(0.58-2.99) \mathrm{p}=0.52$ and HR $=1.11(0.45-2.73) \mathrm{p}=0.82$ respectively]. Similarly, overall survival was not influenced. Finally, mTOR pathway molecules expression was not modified by the presence of the 388Arg allele. Conclusion: The Gly388Arg FGFR4 polymorphism does not seem to have a prognostic value in digestive NETs. In addition, it neither predicts the response to everolimus nor modifies the activation of the mTOR pathway. Keywords: n.

\section{L7 \\ Prognostic Role of Diabetes Mellitus (DM) and Metformin (MET) Therapy in Patients (pts) with Advanced G1-G2 Neuroendocrine Tumors (NETs) Treated with Everolimus (EVE) \\ Custodio A. a, Jiménez-Fonseca P. ${ }^{\mathrm{b}}$, Alonso-Orduña V. López López C. ${ }^{d}$, Alonso T. ${ }^{\text {e }}$, Crespo G. ${ }^{\dagger}$, \\ Carmona-Bayonas A. ${ }^{9}$, Álvarez-Escolá C. ${ }^{\text {h, }}$ Solís M.P. Capdevila J.', Feliu J. a, Aller J.', Grande E. e, Barriuso J. ${ }^{a}$ \\ ${ }^{a}$ Medical Oncology Department Hospital Universitario La Paz, Madrid, Spain; bMedical Oncology Department Hospital Universitario Central de Asturias, Oviedo, Spain; 'Medical Oncology Department Hospital Universitario Miguel Servet, Zaragoza, Spain; ${ }^{d}$ Medical Oncology Department Hospital Universitario Marqués de Valdecilla, Santander, Spain; eMedical Oncology Department Hospital Universitario Ramón y Cajal, Madrid, Spain; ${ }^{\dagger}$ Medical Oncology Department Complejo Asistencial Universitario de Burgos, Burgos, Spain; 'Medical Oncology Department Hospital Universitario Morales Meseguer, Murcia, Spain; hEndocrinology Department Hospital Universitario La Paz, Madrid, Spain; 'Medical Oncology Department Hospital Universitari Vall d'Hebron, Barcelona, Spain; 'Endocrinology Department Hospital Universitario Puerta de Hierro, Majadahonda, Spain}

Introduction: MET has shown antitumorigenic effects on DM cancer pts, which are mediated by decrease of insulin/IGF-1 levels and activation of the AMPK pathway leading to mTOR inactivation. $\operatorname{Aim}(\mathbf{s})$ : To assess the potential prognostic impact of MET administered during EVE therapy among DM pts with advanced NETs. 
Materials and Methods: We retrospectively identified pts with advanced G1-G2 gastroenteropancreatic and lung NETs treated with EVE at 7 Spanish University hospitals. Cox proportional hazard models controlling for primary tumour site, grade and previous therapies were used to compare progression-free survival (PFS) across non-DM pts, DM pts on MET and DM pts on anti-DM agents other than MET. Results: Between June-2009 and May-2014, 92 pts were evaluated, $25(27.2 \%)$ of whom had previous DM (11 treated with MET and 14 with non-MET agents). Median PFS was 14 months (mo) $(95 \%$ CI: 11.4-16.6) in the overall population. In the multivariate analysis, DM pts treated with non-MET agents had poorer outcome than nonDM pts (median PFS: 8.2 and 14.1 mo, respectively; HR: 1.91; 95\% CI: $0.92-3.99 ; \mathrm{p}=0.084)$, while it was significantly better for DM pts on MET (median PFS: 34.1 mo; HR: 0.16; 95\% CI: 0.04-0.68; $\mathrm{p}=0.013$ ). DM pts treated with MET showed improved PFS relative to those receiving non-MET agents (HR: 0.12 ; 95\% CI: 0.02-0.57; $\mathrm{p}=0.008$ ). Conclusion: Our results suggest potential anticancer effects of MET among DM pts with advanced G1-G2 NETs treated with EVE and support further prospective testing in larger cohorts. Keywords: net, diabetes, metformin, everolimus.

\section{L8}

\section{Everolimus (EVE)-Induced Hyperglycemia (HG) in Patients (pts) with Advanced G1-G2 Neuroendocrine Tumors (NETs): Clinical Relevance and Predictive Value}

\author{
Custodio A. a Jiménez-Fonseca P. ${ }^{\mathrm{b}}$, Alonso-Orduña V. ${ }^{\mathrm{c}}$, \\ López López C. ', Alonso T. ${ }^{\mathrm{e}}$, Guillermo C. ${ }^{\dagger}$, \\ Carmona-Bayonas A. ${ }^{\mathrm{g}}$, Álvarez-Escolá C. ${ }^{\mathrm{h}}$, Solís M.P. ${ }^{\mathrm{b}}$, \\ Capdevila J.', Grande E. e, Barriuso J. a, Feliu J. ', Aller J.j \\ ${ }^{a}$ Medical Oncology Department Hospital Universitario \\ La Paz, Madrid, Spain; ' ${ }^{b}$ Medical Oncology Department \\ Hospital Universitario Central de Asturias, Oviedo, Spain; \\ cMedical Oncology Department Hospital Universitario \\ Miguel Servet, Zaragoza, Spain; dMedical Oncology \\ Department Hospital Universitario Marqués de Valdecilla, \\ Santander, Spain; eMedical Oncology Department Hospital \\ Universitario Ramón y Cajal, Madrid, Spain; ' Medical \\ Oncology Department Complejo Asistencial Universitario \\ de Burgos, Burgos, Spain; ${ }^{9}$ Medical Oncology Department \\ Hospital Universitario Morales Meseguer, Murcia, Spain; \\ hEndocrinology Department Hospital Universitario La \\ Paz, Madrid, Spain; 'Medical Oncology Department \\ Hospital Universitari Vall d'Hebron, Barcelona, Spain; \\ 'Endocrinology Department Hospital Universitario Puerta \\ de Hierro, Majadahonda, Spain
}

Introduction: EVE-induced HG can be explained by a decrease in insulin release or increase in peripheral insulin resistance. It has been documented in $12-13 \%$ of NET pts included in phase III clinical trials based on investigators reports. $\operatorname{Aim}(\mathbf{s})$ : To evaluate the clinical relevance and predictive value of EVE-induced HG among pts with advanced NETs. Materials and Methods: Retrospective study of pts with advanced G1-G2 gastroenteropancreatic and lung NETs treated with EVE at 7 Spanish University hospitals. The highest blood sugar level during EVE therapy was considered. A multivariate Cox model controlling for previous diabetes (DM), primary tumour site, grade and prior treatments was used to compare progression-free survival (PFS) across HG CTC grades (gr). Results: Between June-2009 and May-2014, 92 pts were evaluated (25 with previous DM). Any gr HG was observed in $66(71.6 \%)$ pts $(62.1 \%$ gr $1,30.3 \%$ gr $2,7.6 \%$ gr 3). $32(48.5 \%)$ pts needed HG treatment (13 insulin, 20 oral hypoglycemic agents), 25 (78.1\%) of whom experienced improvement to gr $\leq 1$. Neither dose modifications nor discontinuations were required. In the multivariate analysis, median PFS in pts developing $\geq$ gr $2 \mathrm{HG}$ was 39 months (mo) (95\% CI: 13.9-64.2) (HR: 0.28; 95\% CI: 0.09-0.79; $\mathrm{p}=0.017)$, significantly better than in those with gr $1(13 \mathrm{mo}, 95 \% \mathrm{CI}$ : 10.2-15.8) or no HG (11 mo; 95\% CI: 5.1-16.9). Conclusion: Our results confirm that EVE-induced $\mathrm{HG}$ is a common and manageable toxicity and suggest that it may represent a new predictive factor of efficacy. Keywords: net, everolimus, hyperglycemia.

L9

\section{Everolimus in Progressive Metastatic Pancreatic Neuroendocrine Tumors: Analyses of Median Progression Free Survival and Safety in the Real Life Setting}

Del Prete M. ${ }^{\text {a }}$, Malka L. ${ }^{\text {, }}$ Borget I. ${ }^{\text {, }}$ Berdelou A. ${ }^{\text {, }}$ Boige V. ${ }^{\mathrm{b}}$, Leboulleux S. ${ }^{\mathrm{d}}$, Burtin P. ${ }^{\mathrm{b}}$, De Martino M.C. , Faggiano A. ${ }^{a}$, Gianoncelli L. ${ }^{\text {, }}$, Planchard D. ${ }^{f}$, Scoazec J.Y. ${ }^{\mathrm{g}}$, Ducreux M. ${ }^{\mathrm{b}}$, Baudin E. ${ }^{\mathrm{d}}$

aDepartment of Clinical Medicine and Surgery, Section of Endocrinology, Federico II University, Naples, Italy; bDepartment of Medicine, Institut Gustave Roussy, Villejuif, France; 'Department of Biostatistic and Epidemiology, Institut Gustave-Roussy and University Paris-Sud, Villejuif, France; dDepartment of Nuclear Medicine, Institut Gustave-Roussy, Villejuif, France; eDivision of Oncology and Hematology, Humanitas Clinical and Research Center, Rozzano, Milan, Italy; fThoracic Group, INSERM U981, Gustave Roussy; Drug Development Department, Gustave Roussy, Villejuif, France; 'Department of Pathology, Institut Gustave Roussy, Villejuif, France

Introduction: Everolimus is an oral inhibitor of mammalian target of rapamycin (mTOR) with antitumor activity in patients (pts) with pancreatic neuroendocrine tumor (pNET)s. Aim(s): We performed a retrospective analysis to evaluate medians(m) of progression free survival (PFS) and safety in metastatic pNET pts treated with everolimus in the real life setting. Materials and Methods: Consecutive pts with progressive metastatic pNET treated at Gustave Roussy (Villejuif, France) from January 2006 to August 2014 with everolimus were analyzed. Primary end-points were mPFS. Secondary objective were mOS, best response according to Response Evaluation Criteria in Solid Tumor (RECIST v.1.1) and safety according to the National Cancer Institute Common Toxicity Criteria (CTCAE v.4.0). Results: Sixty-six pts were included (27 male-39 female, m: 51 years; range: 22-78). After a m follow-up of 33 months mPFS was 11.1 months (95\%; CI8.6; 18.0) and mOS was 41 months (95\%; CI26.4;66.3). 
Best RECIST response was stabilization ( $76 \%$ pts). Grade $3-4$ toxicity occurred in $31(47 \%)$ pts. Of these $16(24.2 \%)$ experienced more than one grade 3 . Most common grade $3 \mathrm{AE}$ were: hypertension $(\mathrm{n}=$ $15.23 \%)$, hyperglycemia $(n=15.23 \%)$, weight loss $(n=13.20 \%)$, stomatitis $(n=7.10 \%)$, hypertransaminasemia $(n=6.9 \%)$, non-infectious pneumonitis $(\mathrm{n}=5.8 \%)$ leading to drug decrease or discontinuation in 26 or $41 \%$ of cases. Conclusion: We confirm everolimus antitumor activity in metastatic pNET pts. Safety profile of everolimus may constitute a limiting factor in the real world setting. Keywords: pancreatic neuroendocrine tumor, everolimus, safety.

\section{L10 \\ A Randomized Open-Label Phase II Study of Everolimus Alone or in Combination with Pasireotide LAR in Advanced, Progressive Pancreatic Neuroendocrine Tumors (pNET): COOPERATE-2 Trial}

Kulke M.H. ${ }^{\text {a }}$ Ruszniewski P. , Van Cutsem E. c, Valle J. ${ }^{\text {, }}$ De Herder W. ${ }^{\text {, Pavel M. }}$, Degtyarev E. g, Lam D. ${ }^{\text {h, }}$

Salazar R.', Borbath I.j, Fazio N. k, Lombard-Bohas C.!, Yao J.C. ${ }^{\mathrm{m}}$

aDana-Farber Cancer Institute, Boston, USA; bUniversity of Paris VII and Beaujon Hospital, Paris, France; 'University Hospitals Gasthuisberg/Leuven and KULeuven, Leuven, Belgium; dUniversity of Manchester/The Christie Hospital, Manchester, UK; 'Erasmus University Medical Center, Rotterdam, The Netherlands; ${ }^{\dagger}$ Charité Universitätsmedizin, Berlin, Germany; ' Novartis Pharma AG, Basel,

Switzerland; hNovartis Pharmaceuticals Corporation, East Hanover, USA; 'Institut Català d'Oncologia. L'HospitaletBarcelona, Barcelona, Spain; 'Cliniques universitaires Saint-Luc, Brussels, Belgium; kEuropean Institute of Oncology, Milan, Italy; 'Hôpital Edouard Herriot, Lyon, France; mM.D. Anderson Cancer Center, Houston, USA

Introduction: Pasireotide is a somatostatin analog (SSA) that targets somatostatin receptor subtypes 1-3 and 5. An earlier phase I study showed antitumor activity of everolimus + pasireotide LAR (EVE+PAS) in NET. Aim(s): To assess the efficacy and safety of EVE+PAS compared to EVE alone in patients (pts) with advanced, progressive pNET. Materials and Methods: We performed a randomized, open-label, phase II study of EVE \pm PAS in pts with advanced G1/G2 pNET; stratified by prior SSA use, baseline CgA and/or NSE. Primary endpoint was PFS. Secondary endpoints included RECIST-defined tumor response rate, overall survival (OS), and safety. Results: 160 pts were randomized $1: 1$ to EVE $(10 \mathrm{mg} / \mathrm{d}$, oral $)+\operatorname{PAS}(60 \mathrm{mg} / 28 \mathrm{~d}, \mathrm{IM} ; \mathrm{n}=$ $79)$ or EVE $(10 \mathrm{mg} / \mathrm{d}$, oral; $\mathrm{n}=81)$. Pts characteristics: median age $=$ $59 \mathrm{yrs} ;$ males $=54 \%$; WHO PS 0:1 $=64 \%: 34 \%$, prior SSA treatment $=$ $33 \%$. Median PFS was $16.8(12.1-19.6)$ vs. $16.6(11.1-19.5)$ mo in EVE+PAS vs. EVE; HR $=0.99,95 \%$ CI $0.64-1.54$. Partial response was seen in $20.3 \%$ and $6.2 \%$ pts and stable disease in $57 \%$ and $77 \%$ pts treated with EVE+PAS and EVE, respectively. Median OS was not reached in either group. Median treatment duration was 52.1 wks in EVE+PAS and 48.3 wks in EVE arm. Most common adverse events of any grade (EVE+PAS vs. EVE) were hyperglycemia (76\% vs. $27 \%$ ), stomatitis ( $59 \%$ vs. $63 \%$ ), and diarrhea ( $63 \%$ vs. $53 \%)$; grade $3 / 4$ fasting hyperglycemia was seen in $37 \%$ vs. $11 \%$ of pts. Conclusion: In pts with advanced, progressive pNET, addition of PAS to EVE did not significantly improve PFS when compared to EVE alone. Keywords: everolimus, pnet, pasireotide lar.

\section{L11 \\ CBEZ235Z2401: Randomized Phase II Study of BEZ235 or Everolimus (EVE) in Patients with Advanced Pancreatic Neuroendocrine Tumors (pNET)

\author{
Libutti S. ${ }^{\text {a }}$ Garcia-Carbonero R. ${ }^{\mathrm{b}}$, Wolin E.M. ${ }^{\mathrm{c}}$, \\ Custodio A. ${ }^{\text {d }}$, Yao J.C. ${ }^{\mathrm{e}}$, Hendifar A.E. ${ }^{\mathrm{f}}$, Tavorath R. ${ }^{\mathrm{g}}$, \\ Mukherjee N. ${ }^{\text {h }}$, Herbst F., Sulovski J. ${ }^{\text {g, Salazar R. }}{ }^{\mathrm{j}}$
} \\ ${ }^{a}$ Albert Einstein College of Medicine of Yeshiva University, Montefiore Medical Center, New York, N.Y., USA; ${ }^{b}$ Medical Oncology Department, Hospital Universitario Virgen del Rocio, Sevilla, Spain; 'Markey Cancer Center, University of Kentucky, Lexington, Mass., USA; dMedical Oncology Department, Hospital Universitario La Paz, Madrid, Spain; 'Department of Gastrointestinal Medical Oncology, University of Texas M.D. Anderson Cancer Center, Houston, Tex., USA; ${ }^{\dagger}$ Cedars-Sinai Samuel Oschin Comprehensive Cancer Institute, West Hollywood, Calif., USA; 9 Novartis Pharmaceuticals Corporation,

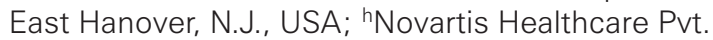 Ltd., Hyderabad, India; 'Novartis Pharma AG, Basel, Switzerland; 'Institut Català d'Oncologia-IDIBELL, L'Hospitalet de Llobregat, Barcelona, Spain}

Introduction: EVE is an mTOR inhibitor (mTORi) approved for the treatment of advanced pNETs. BEZ235, a dual mTOR/PI3K inhibitor, may provide greater PI3K pathway inhibition and enhanced antitumor effects. Aim(s): To present selected secondary endpoints (safety and overall response rate [ORR; RECIST v1.0]) from this open label, randomized, ph II trial (NCT01628913; cut-off Oct 2014). Materials and Methods: Eligible pts had advanced, well-differentiated pNETs, no prior mTORi, and disease progression within the past year. Randomization (1:1) was stratified by somatostatin analog use and baseline chromogranin A and/or neuron specific enolase levels. Pts received BEZ235 (400 mg bid) or EVE (10 mg qd). Results: 62 pts were randomized to BEZ235 or EVE ( $n=31$ each). Pt characteristics were well balanced between arms. At cut-off, $87 \%$ and $71 \%$ of pts in BEZ235 and EVE arms, respectively, had discontinued, mainly due to disease progression (BEZ235: 36\%; EVE: 45\%) and AEs (BEZ235: 39\%; EVE: 16\%). Median duration of treatment was BEZ235: 23 wks and EVE: 39 wks. ORR (95\% CI) was 10\% (2.0-25.8) in both arms. More Gr 3/4 AEs occurred with BEZ235 (84\%) than EVE (71\%). Most common all-grade AEs: diarrhea $(90 \%)$, stomatitis $(74 \%)$, nausea $(55 \%)$, vomiting $(48 \%)$ and asthenia (42\%) with BEZ235; stomatitis (65\%), diarrhea (55\%), asthenia, rash and reduced appetite (42\% each) with EVE. Conclusion: BEZ235 showed some clinical activity in pNET, but due to the drug's safety profile, the study was terminated early. Supported by Novartis Pharma AG. Keywords: pnet, bez235, everolimus. 
L12

\section{A Phase II Study of Everolimus in Patients with FDG-PET Positive Intermediate Grade (Ki67 3-20\%) Pancreatic Neuroendocrine Tumor}

\author{
Michael M. ${ }^{\text {a }}$ Pavlakis N. ${ }^{\mathrm{b}}$, Wyld D. ${ }^{\mathrm{c}}$, Link E. ${ }^{\mathrm{a}}$, Liauw W. ${ }^{\mathrm{d}}$, \\ Kuru N. ${ }^{\text {a }}$ Hicks R. ${ }^{\text {a }}$ \\ apeter MacCallum Cancer Centre, Melbourne, Australia; \\ ${ }^{b}$ Royal North Shore Hospital, Sydney, Australia; 'Royal \\ Brisbane Hospital, Brisbane, Australia; 'St Georges \\ Hospital, Sydney, Australia
}

Introduction: The RADIANT-3 trial showed Everolimus (EV), as active in patients (pts) with progressive well/intermediate grade PNETs. Intermediate/grade 2 (Ki67 3-20\%) PNETs have variable biology. FDG-PET avidity is an independent negative factor for survival, consistent with aggressive disease biology- but such imaging is not used to select treatment as in the case of the RADIANT 3 trial. $\operatorname{Aim}(\mathbf{s})$ : Assess the activity of EV as first-line therapy in patients with FDG-avid intermediate/grade 2 PNETs. Materials and Methods: Eligible pts had newly diagnosed FDG-PET-avid grade 2 advanced PNET with measurable/evaluable disease. ECOG PS 0-2. Adequate organ function. No prior systemic therapy for PNETs. Pts received EV $10 \mathrm{mg}$ po daily until disease progression. Pts were staged every 12 weeks with structural imaging/FDG and Gal-DOTA PET. Primary endpoint was PFS at 6 months. PET/structural imaging response rate, response duration, toxicity and QOL were also measured. Results: 9 pts accrued from Dec 2012. M:F $=5: 4$. Median age $=57$ yrs $(27-$ 66). Median treatment duration $66 \mathrm{wks}$ (range 12-87 wks). 4 pts had PD at 12, 16, 61 and 72 wks. Overall grade (G) 3/4 AEs $\mathrm{N}=7$ pts. EV-related; hyperglycaemia $(\mathrm{G} 3, \mathrm{~N}=2)$ and hypertriglyceridemia $(\mathrm{G} 3, \mathrm{~N}=2)$. EV-related $\mathrm{G} 2$ interstitial pneumonitis requiring dose reduction in $1 \mathrm{pt}$. Best response on $\mathrm{CT} / \mathrm{MRI}$ was $\mathrm{SD}$ in $7 \mathrm{pts}$ and partial FDG-PET response in 2 pts. PFS data to follow. Conclusion: EV in FDG-avid Grade 2 PNETs is tolerable, active with prolonged disease control and requires further evaluation. Keywords: everolimus, fdg-pet.
L13

\section{Clinical Outcome of Everolimus Treatment of Advanced Neuroendocrine Neoplasms: 'Real-Life' Data from the German NET-Register}

Pape U.F.a, Bacher M. ${ }^{\text {a }, ~ F o t t n e r ~ C . ~}{ }^{\text {b }, ~ L a h n e r ~ H . ~}{ }^{c}$, Begum N. ${ }^{\text {, }}$ Quietzsch D. ${ }^{e}$, Skrobek-Engel G. ${ }^{a}$, Pavel M. ${ }^{a}$, Rinke A. ${ }^{\dagger}$, Maasberg S. ${ }^{\text {a }}$

a Medical Department of Gastroenterology and Hepatology, University Medicine Charité, Berlin, Germany; 'bepartment of Endcrinology and Metabolism, University Medicine, Mainz, Germany; ' Department of Endocrinology and Metabolism, University Hospital, Essen, Germany; dDepartment of Surgery, University Hospital Schleswig-Holstein, Campus Lübeck, Lübeck, Germany; ' Department of Internal Medicine, Clinical Centre of Chemnitz, Chemnitz, Germany; 'Department of Gastroenterology and Endocrinology, University Hospital Gießen/Marburg, Campus Marburg, Marburg, Germany

Introduction: Everolimus (EVE) has importantly impacted the treatment (Tx) of mostly pancreatic NEN. Aim(s): This study analyzed recent results of routine EVE-Tx from 5 centres of the German NET-Register. Materials and Methods: Multicentric, retrospective analysis of pts. with histologically proven NEN progressive prior to Tx initiation with EVE (initial diagnosis 1988-2013); statistical analysis used SPSS 19.0. Results: 76 pts. with a median age of 54 years at initial diagnosis were analyzed. Primaries were pancreatic (pNEN, $47 / 62 \%$ ), intestinal (iNEN 14/18\%), bronchopulmonary (bNEN 9/12\%) or unknown (6/8\%). Tx prior to EVE included surgery, chemotherapy, somatostatin analogues, PRRT and ablative Tx. EVE was initiated as 1st-line Tx in 9\%, 2nd-line in $29 \%$, 3rd-line in $20 \%$ and later Tx in $49 \%$. Radiological disease control rate (DCR, i. e. PR and SD) was $72 \%$; no CR was observed. EVE Tx was terminated due to adverse events in 5\%. DCR in 43 pNEN was $65 \%$, in 14 iNEN $100 \%$, in 9 bNEN $78 \%$ and in 6 unknown primaries 50\%. According to Ki67-grading DCR was $83 \%$ in 6 NET-G1, $76 \%$ in 54 NET-G2 and $40 \%$ in 10 NEN-G3. Median overall survival with EVE-Tx was 43 months, while median time-to progression (TTP) was 6, 5.5 and 7 months for all, pNEN and NET-G2 respectively. Conclusion: This analysis shows a reasonable DCR with EVE considering particularly that many pts. (incl. some NEN-G3) had advanced disease with multiple pretreatments ( $>3$ in $49 \%$ ). EVE is now widely used for NEN-Tx although with shorter TTP in later-line Tx. Keywords: everolimus, outcome. 
L14

\section{Recist versus Choi Criteria Response Assesment in Patients with Advanced Pancreatic Neuroendocrine Tumor (PNET) Treated with Sunitinib}

Solis Hernandez M.P. ${ }^{a}$, Calvo-Temprano D. ${ }^{\mathrm{b}}$, Jiménez-Fonseca P. ${ }^{a}$, Faez L. ${ }^{a}$, Ruiz A.L. ${ }^{a}$, Rodriguez D. ${ }^{\text {, }}$ Sanchez M.L. a , Li W. ${ }^{\text {a }}$ Fernandez Arrojo S. ${ }^{a}$, Rodriguez A. , Uriol E. ', Carmona-Bayonas A. ${ }^{c}$, Menendez Prieto M.D. ${ }^{\text {, }}$ Vieitez J.M. ${ }^{\text {a }}$

aDepartment of Oncology. Asturias Central University Hospital, Oviedo, Spain; 'Department of Radiology. Asturias Central University Hospital, Oviedo, Spain; 'Department of Oncology. Morales Meseguer University Hospital, Murcia, Spain

Introduction: Classically, the response assessment to systemic treatment is based on RECIST. In PNET treated with antiangiogenic targeted therapy as sunitinib there is no good correlation between response rate and progression free survival (PFS). Choi criteria might be an alternative to RECIST to evaluate the effect of sunitinib in patients with advanced PNET. Aim(s): To explore Choi as an appropriate response assessment method in PNET treated with sunitinib. Materials and Methods: 17 patients with advanced PNET treated with sunitinib were evaluated by Computed Tomography (CT) using CHOI and RECIST, every 3 months. According to Choi criteria, partial response (PR) was defined as $\geq 10 \%$ decrease in size or $\geq 15 \%$ decrease in attenuation. Results: At 3 months $(n=17)$, according to RECIST 2 patients had PR, 14 stable disease (SD), and 1 progressive disease (PD), whereas according to Choi criteria 12 had PR, 3 $\mathrm{SD}$ and 2 PD. At 6 months $(\mathrm{n}=14)$, according to RECIST 3 had PR, 9 SD, and 2 PD, whereas according to Choi 10 had PR, 2 SD and 2 PD. After progression in 9 patients, PFS was 10.35 vs. 7.50 months in patients who found PR by Choi and RECIST, respectively. Comparing responders and no responders by Choi, PFS was 6.5 vs. 10.3 months, with no statistical significance. Conclusion: Assessment of metastatic PNET target lesions on CT by Choi criteria seems to be more accurate than RECIST. Nevertheless, in PNET treated with sunitinib, the value of Choi for early discriminate patients with better outcome still need to be validated. Keywords: sunitinib, pnet, choi, recist.
L15

\section{Long-Term Follow-Up and Survival Data in Progressing Advanced Midgut Neuroendocrine Tumors Following Peptide Receptor Radionuclide Therapy}

Yalchin M. ${ }^{\text {a }}$ Oliveira A. ${ }^{\mathrm{b}}$, Pencharz D. ${ }^{\mathrm{c}}$, Navalkissoor $S{ }^{\mathrm{c}}$

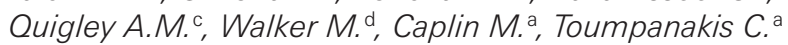

aNeuroendocrine Tumour Unit, ENETS Centre of Excellence, Royal Free Hospital, London, UK; ${ }^{b}$ Care of the Elderly Department, University College Hospital, London, UK; ' Nuclear Medicine Department, Royal Free Hospital, London, UK; 'Faculty of Medicine, St Mary's Campus,

Imperial College London, London, UK

Introduction: Peptide Receptor Radionuclide Therapy (PRRT) is an effective treatment option for advanced Neuroendocrine Tumours (NET), however long term survival data is lacking. Aim(s): To determine, whether there is a difference in long-term survival in patients exhibiting partial response or stable disease, post-PRRT, and to identify prognostic factors for overall (OS) and progression-free survival (PFS). Materials and Methods: Retrospective analysis of OS and PFS (based on radiological RECIST criteria) among a cohort of 133 patients with progressing (despite previous treatments) advanced midgut NET (who received Y-90 or Lu-177 DOTATATE). Univariate Kaplan-Meier techniques and multivariate Cox regression models were used. Results: No statistically significant difference noted in OS of patients with disease stabilization or partial response to PRRT, with median OS of 35 and 47 months respectively (From the date of 1st cycle). Those with progressive disease (one year following PRRT completion) had a statistically significant lower OS, with a median 24 months. More cycles of PRRT (maximum 6) are associated with a statistically significant increase in patient OS, whilst patients having undergone resection of liver metastases and chemotherapy had lower OS. Tumour grade, hepatic load, and carcinoid heart disease affect both PFS and OS. Conclusion: Disease stabilization seems to be a favourable outcome for OS in progressing midgut NET. More studies with larger number of patients are needed to identify definite prognostic factors. Keywords: prrt, pfs, os. 
L16

\section{Everolimus (EVE) for Advanced, Progressive Pancreatic Neuroendocrine Tumors (pNET): Final Overall Survival (OS) from a Randomized, Double-Blind, Placebo (PBO)-Controlled, Multicenter Phase 3 RADIANT-3 Study}

Yao J.C. , Pavel M. ${ }^{\text {b }}$ Lombard-Bohas C. ${ }^{\text {, }}$, Van Cutsem E. ${ }^{\text {, }}$ Lam D. ${ }^{\mathrm{e}}$, Kunz T. ${ }^{\mathrm{e}}$, Brandt U. ${ }^{\mathrm{f}}$, Capdevila J. ${ }^{\mathrm{g}}$, De Vries E. Tomassetti P., Hobday T. ${ }^{\mathrm{j}}$, Pommier R. ${ }^{\mathrm{k}}$

aDepartment of Gastrointestinal Medical Oncology, The University of Texas MD Anderson Cancer Center, Houston, Tex., USA; 'bepartment of Hepatology and Gastroenterology, Charité Universitätsmedizin Berlin/ Campus Virchow Klinikum, Berlin, Germany; ' Department of Medical Oncology, Hôpital Edouard Herriot, Hospices Civils de Lyon, Lyon Cedex 03, Lyon, France; dDigestive Oncology, University Hospitals Gasthuisberg/Leuven and KULeuven, Leuven, Belgium; ' Novartis Pharmaceuticals Corporation, East Hanover, N.J., USA; ' Novartis International AG, Basel, Switzerland; ' ${ }^{\text {Department of }}$ Medical Oncology, Vall d'Hebron UniversityHospital, Barcelona, Spain; ' Department of Medical Oncology at the UMCG, Groningen, The Netherlands; 'Department of Medical and Surgical Sciences University of Bologna, Bologna, Italy; 'Department of Oncology, Mayo Clinic College of Medicine, Rochester, N.Y., USA; kDivision of Surgical Oncology, Oregon Health \& Science University, Portland, Oreg., USA

Introduction: EVE showed a significant improvement of $6.4 \mathrm{mo}$ in median progression-free survival (PFS) vs. PBO (HR 0.35, 95\% CI $0.27-0.45 ; \mathrm{P}<0.001$ ) in patients (pts) with pNET in RADIANT-3. $\operatorname{Aim}(\mathbf{s})$ : To report final OS and safety updates from RADIANT-3. Materials and Methods: 410 pts with advanced, progressive, low-/ intermediate-grade pNET were randomized to EVE $10 \mathrm{mg} / \mathrm{d}(\mathrm{n}=$ $207)$ or PBO $(n=203)$ both with best supportive care. Crossover from PBO to open-label EVE upon disease progression was allowed in double-blind phase. At the end of core phase, ongoing patients from both arms were switched to open-label EVE at investigator's discretion after unblinding. After 256 events, OS was analyzed by stratified log-rank test. OS by baseline CgA levels (low $[\leq 2 x U L N]$ vs. high [ $>2 \mathrm{xULN}]$ ) was compared. Results: 225 pts received open-label EVE; $172(85 \%)$ switched from PBO and 53 (26\%) from EVE arm. Median OS (95\% CI) was 44.0 (35.6-51.8) mo for pts initially randomized to EVE and 37.7 (29.1-45.8) mo for those randomized to PBO (HR 0.94, 95\% CI 0.73-1.20; P=0.30). Low baseline CgA was a prognostic marker for OS irrespective of treatment (HRlow/high $0.54 ; 95 \%$ CI, 0.42-0.70). Stomatitis (47\%), diarrhea (44\%), and rash (40\%) were most common AEs (open-label phase). Conclusion: Median OS of 44 mo with EVE is noteworthy in advanced, progressive pNET. Survival benefit of 6.3 mo with EVE vs. PBO is consistent with PFS improvement reported earlier. Crossover of majority of pts $(85 \%)$ may have confounded OS. Safety profile was consistent. Keywords: everolimus, pnet, os.

\section{Medical Treatment - Others}

\section{M1 \\ Niacin (vitamin B3) Suppletion in Patients with Serotonin Producing Neuroendocrine Tumors}

Bouma G., Van Faassen M., Kats-Ugurlu G., De Vries E., Kema I., Walenkamp A.

University Medical Center Groningen, Groningen, The Netherlands

Introduction: Tryptophan is the precursor of serotonin and niacin (vitamin B3), critical for normal cellular metabolism. Tryptophan and niacin can be deficient in patients with serotonin producing neuroendocrine tumors (NETs). Niacin deficiency can lead to symptoms including pellagra. Aim(s): To assess niacin status before and after suppletion in these patients. Materials and Methods: We identified serotonin producing NET patients who had received oral niacin suppletion for tryptophan deficiency and/or pellagra associated symptoms. Pre-suppletion plasma tryptophan levels and niacin status based on the urinary niacin metabolite N1-methylnicotinamide (N1$\mathrm{MN})$ before $(\mathrm{n}=42)$ and after start suppletion (in 34 paired samples) were assessed. Results: The mean pre-suppletion plasma tryptophan level was $31.8 \pm 9.7 \mu \mathrm{mol} / \mathrm{L}$ (reference value $40.0-70.0 \mu \mathrm{mol} / \mathrm{L}$ ). Presuppletion urinary N1-MN levels were lower in patients (median 17.9 $\mu \mathrm{mol} / 24$-hour (24-h), range 2.6-70.3) compared to healthy controls (median $43.7 \mu \mathrm{mol} / 24-\mathrm{h}$, range 9.5-169.3, $\mathrm{P}<0.0001$ ) and below normal in $45 \%$ of the patients. Niacin suppletion increased urinary N1-MN levels to high normal levels (median $55.5 \mu \mathrm{mol} / 24-\mathrm{h}$, range $7.4-489.0)$ in $86 \%$ of the niacin deficient patients. Conclusion: In serotonin producing NET patients niacin deficiency is prevalent. Therefore, urinary N1-MN deserves to be included in their standard biochemical evaluation. Niacin suppletion normalizes niacin status in most niacin deficient serotonin producing NET patients. Keywords: net, serotonin, niacin, n1-methylnicotinamide.

\section{M2 \\ Revalidation of QLQC30 and GINET21 Module in Patients with Neuroendocrine Tumors Using Online Questionnaire}

\author{
Kaupp-Roberts S. ${ }^{\text {a }}$ Gray D. ${ }^{\text {, }}, O^{\prime}$ Donnell K. ${ }^{\text {a }}$, Bouvier C. ${ }^{\text {, }}$ \\ Ramage J. ${ }^{\text {a }}$ \\ aHampshire Hospitals NHS Foundation Trust, Basingstoke, \\ UK; bUniversity of Winchester, Winchester, UK; 'NET \\ Patient Foundation, Hockley Heath, UK
}

Introduction: The GINET21 module measures PROMS for patients with NETs and was validated from 2006, since when there have been rapid changes in cancer therapeutics. Current module revalidation methodology a time and resource-intensive process, 
which may lag behind these treatment. $\operatorname{Aim}(\mathbf{s})$ : Online questionnaires may be an efficient method of achieving similar results in a shorter time-frame. Materials and Methods: 205 participants completed an online questionnaire between October 2013 and February 2014. Patients were asked to gauge the relevance of all QLQ C30 and GINET21 questions on a Likert scale of 1 to 4 , and to complete 3 free text questions inviting them to raise issues not explored by the QLQ C30 and GINET21. Questions were assigned a mean relevance score (s), and ranked by relevance. Results: C30 Q5 failed to attain the EORTC cut-off of $\mathrm{s}=2.00$. All GINET21 questions scored above 2.00, the lowest being Q21 ( $\mathrm{s}=2.14)$. There were no significant differences between pancreatic NET $(\mathrm{n}=36)$ and non-pancreatic NET $(n=169)$ patients. Analysis of the free text items indicated that patients felt that their condition was poorly understood. Conclusion: Results suggest that the QLQ C30 and GINET21 remain relevant and valid, with only one question meeting exclusion criteria. There appears to be no requirement for a separate pancreatic NET module. The online questionnaire managed to reach a large number of patients in a short timeframe, and has generated similar results to previous studies. It warrants consideration as a method for future research. Keywords: qol prom.

\section{M3 \\ Is There an Additional Value of Somatostatin Receptor Subtype 2A Immunohistochemistry Over Somatostatin Receptor Scintigraphy in Predicting Gastroenteropancreatic Neuroendocrine Tumor Response?}

Van Adrichem R., Kamp K., Van Deurzen C., Biermann K., Feelders R.A., Kwekkeboom D., Hofland L.J., De Herder W.

Erasmus Medical Center, Rotterdam, The Netherlands

Introduction: It is not known whether tumoral somatostatin receptor subtype 2a (sst2a) immunohistochemistry (IHC) has additional value over OctreoScan ${ }^{\circledR}$ uptake in predicting response to PRRT in patients with gastroenteropancreatic neuroendocrine tumors (GEP-NETs). Aim(s): The aims were to: 1) establish sst2a positivity in GEP-NET samples of patients who had been treated with PRRT using 177Lu-octreotate, 2) determine the relationship between GEPNET response to PRRT and tumoral sst2a expression, and 3) compare patient's characteristics with sst2a negative and positive tumors. Materials and Methods: We have selected 75 consecutive GEPNET patients who had undergone PRRT using 177Lu-octreotate and who had follow-up by computed tomography (CT). Tumoral sst2a expression was determined by IHC. Tumor response to CT scans was scored according to Response Evaluation Criteria in Solid Tumors (RECIST) criteria. Results: Sst2a positivity was observed in $93 \%$ of tumor samples. No statistically significant relationship was found between sst2a expression and GEP-NET response to PRRT with 177Lu-octreotate. Patients characteristics [sex, age at diagnosis, primary tumor, disease stage, TNM classification, Ki-67 index, highest serum chromogranin-A (CgA) level, and highest neuron specific enolase level (NSE)] were not significantly different between patients with sst2a negative and positive tumors. Conclusion: In conclusion, there is no additional value of determining tumoral sst2a expression over OctreoScan ${ }^{\circledR}$ uptake in predicting GEP-NET response after PRRT. Keywords: sst2a.

M4

Telotristat Etiprate in a Subset of Carcinoid Syndrome Patients Who Have High Levels of Urinary 5-Hydroxyindoleacetic Acid and Frequent Flushing

\author{
Wheeler D. ${ }^{a}$, Hörsch D. ${ }^{\mathrm{b}}$, Valle J. ${ }^{\mathrm{c}}$, Lapuerta P., \\ Zambrowicz B. ${ }^{a}$, Sands A. ${ }^{\text {a }}$ Fleming $D$. $^{\text {a }}$ \\ aLexicon Pharmaceuticals, Inc., The Woodlands, USA \\ bZentralklinik Bad Berka, Bad Berka, Germany; ${ }^{\text {cTTe }}$ \\ University of Manchester/The Christie NHS Foundation \\ Trust, Manchester, UK
}

Introduction: Serotonin is a key mediator of carcinoid syndrome (CS). CS patients (pts) with high levels of urinary 5-hydroxyindoleacetic acid (u5-HIAA, a serotonin metabolite) and $>3$ flushing episodes/day are at increased risk of developing carcinoid heart disease (CaHD). Aim(s): Telotristat etiprate (TE) is an oral inhibitor of serotonin synthesis in Phase 3 development for the treatment of CS. There is limited information on the potential benefits of serotonin reduction in CS pts who may be at higher risk for CaHD. Materials and Methods: We retrospectively reviewed data on all pts who received TE in a Phase 2 trial for CS. In pts with elevated baseline u5-HIAA and $>3$ flushing episodes/day, we assessed reductions in u5-HIAA, bowel movements (BMs) and flushing frequency while on TE. Results: 5 (33\%) of the 15 enrolled pts met the criteria, including a patient diagnosed with CaHD 1 month prior to study entry. All pts had elevated baseline u5-HIAA levels (mean $97 \mathrm{mg} / 24 \mathrm{hrs}$, range 11-282) and inadequately controlled CS ( $\geq 4$ daily BMs and $\geq 3$ flushing episodes/day), despite use of somatostatin analogs (3/5 patients). At week 12, u5-HIAA levels were reduced (mean 67\%, range 44-97\%). There was also reduction in BM frequency (mean $52 \%$, range $32-72 \%$ ) and daily flushing (mean $45 \%$, range 3-86\%). There was $1 \mathrm{SAE}$ of gastroenteritis, not considered drug-related. Conclusion: In CS pts who may be at risk for developing CaHD, u5-HIAA levels, flushing, and BM frequency all decreased after 12 weeks of TE treatment. Keywords: biomarker, carcinoid heart disease. 
M5

\section{Transcatheter Arterial Chemoembolization for Unresectable Metastatic Hepatic Lesions of Pancreatic Neuroendocrine Tumors}

\author{
Xin B. ${ }^{a}$, Lou W. ${ }^{a}$, Ji Y. ${ }^{b}$, Liu L.X. ${ }^{c}, X u X^{a}{ }^{a}$ \\ aDepartment of Pancreatic Surgery, Zhongshan Hospital, \\ Fudan University, Shanghai, China; 'bepartment of \\ Pathology, Zhongshan Hospital, Fudan University, \\ Shanghai, China; 'Department of Interventional Radiology, \\ Zhongshan Hospital, Fudan University, Shanghai, China
}

Introduction: The blood supply of metastatic hepatic neuroendocrine tumors is mainly supplied by hepatic arteries. For unresectable metastatic NETs, selective embolisation of the hepatic arteries may be therapeutic. Aim(s): To operate transcatheter arterial chemoembolization for metastatic hepatic NETs of pancreas origin and observe its value. Materials and Methods: The clinical and pathological datas of 27 patients who were performed TACE in our hospital from Sep 2004 to Dec 2013 were retrospectively analysed. Results: The cohorts contain 14 males and 13 females, the average age was 52.8-year-old. Pancreatectomy was implemented on 15 patients. Morphologically, well-, intermediately and poor differentiation ratios were $18.5 \%, 44.4 \%$ and $37 \%$, respectively. According to the WHO standard, G1, G2 and G3 accounted for $14.8 \%, 51.9 \%$ and $29.6 \%$, respectively, whereas 1 case was unknown. The mean of Ki-67 index was $15.82 \%$. The percentages of main vascular invasion, portal veinemboli and lymph node metastases were $18.5 \%$, respectively. $26 \%$ patients relapsed and the mean relapse time was 23-mo. Overall survival rates of 3 -year and 5 -year were $74 \%$ and $52 \%$. The median survival time was 46.5 -mo. Age $\geqq 60$-year, higher grade, Ki-67 index $\geqq 10 \%$ and poor differentiation were dismal predictors $(\mathrm{P}<0.05$ ). Patients who underwent TACE more than four sessions survived longer $(\mathrm{P}=0.007)$. Conclusion: TACE demonstrated its effictiveness for unresectable metastatic hepatic NETs. Patients with higher age, higher Ki-67 index may hint the poor outcome. Keywords: pnet, hepatic lesion, tace.

\section{M6 \\ The Role of External Beam Radiotherapy in the Management of Advanced Neuroendocrine Tumors}

Yalchin M. ${ }^{a}$, Tharmalingam $H{ }^{b}$, Owen $C^{b}{ }^{b}$, Walker M. ${ }^{c}$, Caplin M. ${ }^{\text {, Stewart G. }}{ }^{\mathrm{b}}$, Toumpanakis C. $^{\mathrm{a}}$

aNeuroendocrine Tumour Unit, ENETS Centre of Excellence, Royal Free Hospital, London, UK; ' Oncology Department, Royal Free Hospital, London, UK; 'Faculty of Medicine, St Mary's Campus, Imperial College London, London, UK

Introduction: Palliative external beam radiotherapy (EBRT) is routinely used to control symptoms and disease progression in metastatic malignancies, but there is limited data in metastatic neuroendocrine tumours (NET). Aim(s): Compare symptom-free (SFS) and progression-free survival (PFS) of patients with advanced NET who received EBRT and identify prognostic factors. Materials and Methods: Forty five patients were included and analysed retrospectively. Symptoms were assessed via a score and radiological assessment was based on cross-sectional imaging. Univariate KaplanMeir techniques and multivariate regression models were utilized. Results: EBRT was used mainly for symptom control (pain/neurological signs). $75 \%$ of target lesions were skeletal metastases and $25 \%$ soft tissue masses. $42 \%$ of tumours were foregut origin, $29 \%$ unknown primary, $18 \%$ midgut, $9 \%$ hindgut, and $2 \%$ other. Overall, median SFS and PFS were 4 and 4 months respectively. SFS and PFS were statistically significantly shorter in patients with liver metastases compared to those without (median 3 vs. $5 \& 7$ months). Consistent with other series, a statistically significant association between higher radiotherapy (RT) doses per fraction and reduced SFS and PFS was noted. In patients who had post-EBRT Peptide Receptor Radionuclide Therapy (PRRT) SFS and PFS was longer (7 \& 10 months) vs. 4 months in patients who did not have PRRT. Conclusion: EBRT seems to control temporarily symptoms and tumour growth. Hepatic metastases, RT dose and post-EBRT PRRT can affect SFS and PFS. Keywords: ebrt, net.

\section{PRRT-Ablative Therapies-Endoscopic Treatment}

N1

Radioembolization with 90Y-Labelled
Resin Microspheres in Patients with Liver
Metastases from Neuroendocrine Tumors

Ebeling-Barbier C. ${ }^{a}$, Garske-Roman U. ${ }^{\text {, }}$, Antonodimitrakis $P^{c}$, Sandström M. ${ }^{\mathrm{d}}$, Nyman R. ${ }^{\mathrm{a}}$, Granberg D. ${ }^{c}$

aDepartment of Radiology, Uppsala, Sweden; 'bepartment of Nuclear Medicine, Uppsala, Sweden; ' Department of Medical Sciences, Department of Endocrine Oncology, Uppsala, Sweden; dDepartment of Medical Physics, Uppsala, Sweden

Introduction: Hepatic arterial embolization with 90Y-labelled microspheres is a method of delivering radioactivity to liver tumors, that has been used to treat primary hepatocellular carcinomas and liver metastases from colorectal carcinomas and neuroendocrine tumors. $\operatorname{Aim}(\mathbf{s})$ : To evaluate the efficacy and safety of radioembolization in patients with neuroendocrine tumors. Materials and Methods: 38 patients with progressing neuroendocrine liver metastases underwent 51 embolizations with 90Y-labelled resin microspheres (SIR-Spheres ${ }^{\circledR}$ ). 31 embolizations were performed in the right, 11 in the left and 9 in both liver lobes. Mean activity was 1.3 GBq. Evaluation was performed with CT or MRI according to modified RECIST criteria. Results: At the first evaluation after 49 embolizations, after mean 3 months (2-7), PR was seen in 26 lobes (53\%), $\mathrm{SD}$ in $21(43 \%)$ and $\mathrm{PD}$ in 2 lobes (4\%). At the final evaluation mean 
19 months after the first embolization, 1 patient (3\%) had CR, 13 (33\%) had PR, 13 (33\%) showed SD and 12 (31\%) had PD. In the 11 patients with initial PR or SD and PD at the final evaluation, mean time-to-progression was 16 months (7-30). Adverse effects were mild, except that one patient, who did not comply to abstaining from alpha-interferon after the embolization, died from radiation induced hepatic failure. Conclusion: Liver embolization with 90Y-labelled resin microspheres (SIR-Spheres ${ }^{\mathrm{TM}}$ ) is an effective, well-tolerated therapy for patients with progressing neuroendocrine liver metastases. Keywords: radioembolization, neuroendocrine, liver metastases.

\section{N2 \\ Selective Internal Radiation Therapy (SIRT) Radioembolisation with 90 Yttrium in Patients with Unresectable Liver Neuroendocrine Metastases in a Specialized Center. An Initial Report}

García Mónaco R. ${ }^{a}$, Peralta O. ${ }^{a}, O^{\prime}$ Connor J.M. ${ }^{b}$, Pesce V. ${ }^{c}$, Bestani C. ${ }^{c}$, Roca E. ${ }^{\mathrm{c}}$

aHospital Italiano Buenos Aires, CABA, Argentina;

${ }^{b}$ Alexander Fleming Institute, CABA, Argentina;

${ }^{\circ}$ Gastroenterology Hospital B. Udaondo, CABA, Argentina

Introduction: Most patients with neuroendocrine tumors present liver metastases at some point of the clinical course. Liver directed treatment, such as 90 Yttrium is a method of treatment used in patients with non-resectable liver lesions, which is effective in the control of symptoms and tumor growth. $\operatorname{Aim}(\mathbf{s})$ : To describe clinical outcome of patients treated with 90 Yttrium embolisation, in term of toxicity and efficacy. Materials and Methods: A prospectively collected database of 68 patients treated with SIRT spheres, with different etiologies, was analysed. We reviewed 11 patients with liver neuroendocrine metastases, who underwent 90Yttrium embolisation procedures. Results: neuroendocrine tumors originated from the small bowell $54 \%$ were included. The median age was 62 years at treatment. According to WHO criteria all were well-differentiated neuroendocrine tumors. Clinical response was assessed using WHO and RECIST criteria. Seventy-two percent were clinical responders (partial response and stable disease) and more than $60 \%$ of the cases showed control of symptoms. There was no acute toxicity during procedures. No radiation liver failure occurred. Fatigue and increase of liver enzymes were the most frequent toxicities. Conclusion: This first report showed that this treatment is feasible, with low morbimortality. Radioembolisation 90 Yttrium is an effective therapeutic option in patients with unresectable liver metastases. Keywords: 90yttrium, radioembolization, neuroendocrine tumors.

\section{N3 \\ Efficacy of PRRT in Metastatic Neuroendocrine Tumors; A Retrospective Large Single Institutional Study}

\author{
Jan H., Wyld D., Burge M.
}

Royal Brisbane and Women's Hospital, Brisbane, Australia

Introduction: PRRT is a well-documented and well-established treatment for metastatic neuroendocrine tumours. However retrospective studies detailing the efficacy of PRRT are lacking. Aim(s): This study aims to retrospectively analyse factors that affect increased survival and improve QOL in patients who receive PRRT. Materials and Methods: The study includes a total of 57 patients who were treated at the Royal Brisbane and Women's Hospital in the last 5 years with PRRT. Data were collected by studying individual patient records and charts. The factors studied include presence or absence of liver metastases, GE vs. pancreatic histology, Ki 67, number of cycles and dose of PRRT, adverse effects, delays in treatment and improvement of QOL and PFS. In addition OS is being calculated using historical controls. Results: Of the 57 patients $42 \%$ were pancreatic neuroendocrine and the rest were GI primary. $90 \%$ of patients who received PRRT had symptomatic liver metastases. $80 \%$ of patients received 4 cycles of Lutetium and $20 \%$ received only three cycles of treatment. There was objective response in terms of tumour dimensions in just under $80 \%$ of patients. The PFS is 42 months as compared to historical controls. $10 \%$ of patients had repeat treatment with PRRT. Grade 3-4 Hematologic toxicity occurred in $3.5 \%$ of patients. The Overall Survival is being calculated. Conclusion: PRRT is an efficacious treatment for metastatic neuroendocrine tumours with large improvements in objective response and PFS. Keywords: lutetium, somatostatin receptor, prrt, neuroendocrine tumors.

\section{N4 \\ 177Lu Dotatate vs. Sequential 177Lu Dotatate and 90Y Dotatate: Treatment Outcomes for PRRT Patients at the Christie - A Clinical Audit}

\author{
Page E., Tipping J., Birch E., Hamilton D., Manoharan P. \\ The Christie NHS Foundation Trust, Manchester, UK
}

Introduction: The traditional treatment regime of somatostatin receptor positive NETs by molecular radiotherapy was based on single radionuclides (e.g. 90 Y Dotatate or $177 \mathrm{Lu}$ Dotatate), delivered as multiple cycles to allow normal tissue recovery. However a newer practice of alternating tcycles of $177 \mathrm{Lu}$ and Y90 Dotatate was implemented at the Christie following introduction of this regime in some European centres. Subsequent follow up studies have indicated that longer overall survival and reduced loss of kidney function is found in patient cohorts treated with combined $177 \mathrm{Lu}$ and $90 \mathrm{Y}$ regimes to either $177 \mathrm{Lu}$ or $90 \mathrm{Y}$ alone. Aim(s): An audit was conducted at our centre of patient responses to PRRT to determine whether the combined radionuclide regime was delivering equivalent outcomes to the all Lutetium regime given to the earlier patients, without any addi- 
tional toxicities. Materials and Methods: Comparisons were made of haematological toxicity, renal toxicity and other side effect such as nausea, lethargy, diarrhoea, carcinoid symptoms. Also red marrow dose was estimated from blood samples for all patients and related to blood count response. Results: In each case attempts have been made to relate the responses seen not only to the current treatment but to also consider the disease state and patient pre-treatment characteristics which may predict blood and renal toxicity. Conclusion: Finally (where data exists) the long term disease response was evaluated in terms of morphological, symptomatic and biochemical response. Keywords: prrt, toxicity, audit, rm dose.

\section{N5 \\ Evaluation of Features and Predictors for Long Term Hematologic Toxicity in Neuroendocrine Patients Treated with Peptide Receptor Radionuclide Therapy}

Richter S., Xenocostas A., Sanatani M., Davidson R., Novak K., Quan D., Mujoomdar A., Gray D., Laidley D., Bryanton M., Ernst S., Kocha W., Reid R.H.

London Health Sciences Centre, London, Canada

Introduction: Peptide receptor radionuclide therapy (PRRT) for neuroendocrine tumors (NET) may offer partial or complete objective response in up to $30 \%$ of patients. To deliver treatment in a safe and effective manner, long term adverse events should be considered. $\operatorname{Aim}(\mathbf{s})$ : Our objective was to evaluate the clinical characteristics of PRRT patients who developed secondary hematologic malignancies (HM) as a long-term toxicity. Materials and Methods: Patients (pts) eligible for evaluation received PRRT for unresectable NETs between April 1997-December 2010. Parameters including age, gender, time interval from diagnosis to hematologic malignancy, total cumulative radiolabel dose $(\mathrm{GBq})$, and treatments were analyzed by descriptive statistics. Comparative analysis was performed using t- and z-tests. Results: A total of 172 pts were eligible for evaluation. All pts received at least one cycle of MIBG, Indium-111 or Lutetium-177. Of those, 3.5\% $(n=6)$ pts had HM (4 acute myeloid leukemias and 2 myelodysplastic syndromes). Mean interval from NET diagnosis to HM was 7.5 years. Mean age of NET diagnosis was $36.0 \mathrm{HM}(\mathrm{p}=0.02)$. The male:female ratio was lower for patients with HM (1:5) compared to those without $(85: 87)(\mathrm{p}=0.057)$. Patients with HM received a mean cumulative dose of 59.4 GBq (32.1-96.6 $\mathrm{GBq})$. Two pts received concurrent chemotherapy with epirubicin. Conclusion: The rate of HM was higher than in phase I study but similar to rates reported in case series. This finding is compounded by concurrent use of chemotherapy. Keywords: prrt.
N6

\section{FDG PET as a Prognostic Factor in Pancreatic Neuroendocrine Tumors (P-NET) after Peptide Receptor Radionuclide Therapy with 177Lu-Dotatate (Lu-PRRT)}

\author{
Sansovini M. ${ }^{a}$, Stefano S. ${ }^{a}$, lanniello A. ${ }^{a}$, Nicolini S. ${ }^{\text {, }}$ \\ Fantini L. a, Bongiovanni A. b, Di lorio V. ', Sarnelli A. d, \\ Monti M. e, Paganelli G. ${ }^{\text {a }}$
}

aNuclear Medicine Unit, Istituto Scientifico Romagnolo per lo Studio e la Cura dei Tumori (IRST) IRCCS, Meldola (FC), Italy; ' Osteoncology Unit, Istituto Scientifico Romagnolo per lo Studio e la Cura dei Tumori (IRST) IRCCS, Meldola (FC), Italy; ' Pharmacy Unit, Istituto Scientifico Romagnolo per lo Studio e la Cura dei Tumori (IRST) IRCCS, Meldola (FC), Italy; dPhysics Unit, Istituto Scientifico Romagnolo per lo Studio e la Cura dei Tumori (IRST) IRCCS, Meldola (FC), Italy; eBiostatistics Unit, Istituto Scientifico Romagnolo per lo Studio e la Cura dei Tumori (IRST) IRCCS, Meldola (FC), Italy

Introduction: The best-known altered metabolism in cancer is an increased glycolysis. FDG-PET allows us to obtain in vivo imaging of this metabolic abnormality which is an hallmark of tumor aggressiveness. Aim(s): Investigated the role of FDG PET as a prognostic factor P-NET treated with Lu-PRRT. Materials and Methods: A total of 65 G1-G2 P-NET patients (pts), with documented radiological progression, were treated with 2 different activities of $18.5 \mathrm{GBq}$ or $27.8 \mathrm{GBq}$ according to the pts's kidney function and bone marrow reserve. 31 received a total activity (TA) of $27.8 \mathrm{GBq}$ and 34 a TA of 18.5GBq. TA was divided into 5 cycles, $6-8$ weeks apart. 56 of 65 pts underwent a FDG PET before Lu-PRRT. Results: 32 pts were FDG positive, the remaining 24 pts were FDG negative. Regardless the TA of Lu-PRRT, in FDG positive group the Disease Control Rate (DCR), was $84 \%$, with $25 \%$ partial response (PR) and $54 \%$ stable disease (SD). Whereas, the DCR in pts FDG negative was equal to $100 \%$, with $12.5 \%$ of CR, $21 \%$ of PR and $66.5 \%$ of SD. Of note, the median PFS in the FDG positive group was 23 mo (19-29), while in the negative FDG group was 69 mo $(49-69)(p<0.0001)$. Conclusion: FDG PET is a strong prognostic factor in pts with advanced well differentiated P-NET. PRRT is a valid and safe therapeutic approach in P-NET even at the TA of $18.5 \mathrm{GBq}$ in 5 cycles in pts at higher risk for toxicity. Accordingly, FDG positive pts should be treated with a more aggressive therapeutic approach, based on the association of Lu-PRRT with chemotherapy. Keywords: prrt, neuroendocrine, fdg-pet. 
N7

\section{Lu-Dotatate Re-Treatment in G1-G2 Gastro-Entero-Pancreatic (GEP) NETs}

Stefano S. ${ }^{a}$, Maddalena S. ${ }^{a}$, lanniello A. ${ }^{a}$, Nicolini S. ${ }^{\text {, }}$ Caroli P. ${ }^{\mathrm{a}}$ Ibrahim T. ${ }^{\mathrm{b}}$, Donati C. ${ }^{\mathrm{c}}, D^{\prime}$ Errico V. ${ }^{\mathrm{d}}$, Monti M. ${ }^{\mathrm{e}}$, Paganelli G. $^{\text {a }}$

aNuclear Medicine Unit, Istituto Scientifico Romagnolo per lo Studio e la Cura dei Tumori (IRST) IRCCS, Meldola

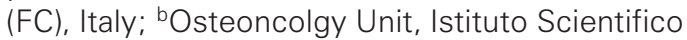
Romagnolo per lo Studio e la Cura dei Tumori (IRST) IRCCS, Meldola (FC), Italy; 'Pharmacists Unit, Istituto Scientifico Romagnolo per lo Studio e la Cura dei Tumori (IRST) IRCCS, Meldola (FC), Italy; dPhysics Unit, Istituto Scientifico Romagnolo per lo Studio e la Cura dei Tumori (IRST) IRCCS, Meldola (FC), Italy; eBiostatistics Unit, Istituto Scientifico Romagnolo per lo Studio e la Cura dei Tumori (IRST) IRCCS, Meldola (FC), Italy

Introduction: PRRT is a valid and well tollerated therapy for G1-G2GEP-NETs. A median PFS of $>22$ mo. has been reported after PRRT and OS of $>5$ years is frequent. Aim(s): Evaluation of toxicity and objective response (OR) of re-treatment with $177 \mathrm{Lu}$-dotatate (Lu-PRRT) in GEP NET responders to 90Y-Dotatoc (Y-PRRT). Materials and Methods: 26 pts were consecutively enrolled into a phase II study of re-treatment with Lu-PRRT. All had previously undergone treatment with Y-PRRT and overall outcome was $1 \mathrm{CR}, 3$ PR and 22 SD. PFS was 28 mo. (95\% CI 20-36). After confirmation of disease progression, pts received $14.8-18.5 \mathrm{GBq}$ of Lu-PRRT in $4 / 5$ cycles. All had conserved kidney and hematological parameters before starting Lu-PRRT. Results: Pts received a median total activity of $16.5 \mathrm{GBq}$ in 5 cycles. ORs were $1 \mathrm{CR}, 1 \mathrm{PR}, 20 \mathrm{SD}$ and 4 PD. Median PFS was 22 mo. (95\% CI 16-not reached). There was no significant difference in PFS between Y-PRRT and Lu-PRRT re-treatment. Toxicity was very mild after Lu-PRRT, only 2 pts experiencing grade 2 and 1 pt grade 3 bone marrow toxicity. One pt reported grade 2 and another grade 3 renal toxicity. Tumor burden and number of liver metastases were important predictive markers for OS. Conclusion: G1-G2 GEP NET responders to Y-PRRT are candidates for Lu-PRRT re-treatment after progression. In our pts low-dose Lu-PRRT was safe and obtained OR and PFS rates comparable with those of Y-PRRT. Keywords: prrt, gep-net, re-treatment.

\section{Surgical Treatment}

\section{1 \\ Risk Factors for Survival from Neuroendocrine Neoplasia with Unknown Primary Tumor Site}

Begum N. ${ }^{a}$, Wellner U. ${ }^{\text {a }}$, Thorns C. ${ }^{\text {, }, H o f f m a n n ~ M . ~}{ }^{\text {a }}$ Bürk C. ${ }^{\mathrm{c}}$, Brabant G. ${ }^{\mathrm{d}}$, Lehnert H. ${ }^{\mathrm{d}}$, Keck T. ${ }^{\mathrm{a}}$

aUKSH Lübeck, Department for General Surgery, Lübeck, Germany; 'UKSH Lübeck, Institute for Pathology, Lübeck, Germany; 'Hospital Stadthagen, Stadthagen, Germany; dUKSH Lübeck, Department for Medicine I, Lübeck, Germany

Introduction: Neuroendocrine neoplasia (NEN) with unknown primary site (NEN-CUP) may have a poor prognosis. We evaluated the clinical presentation, therapy, outcome, and risk factors for adverse outcomes in patients with NEN-CUP. Aim(s): Role of surgery in NEN-CUP. Materials and Methods: In 243 patients who had NEN, a retrospective review was performed in 38 patients who had NEN-CUP tumors. The 38 patients who had NEN-CUP were evaluated in 3 groups: group 1 (surgery; primary tumor detected; $\mathrm{n}=$ 10); group 2 (surgery; no primary tumor detected; $\mathrm{n}=10$ ); and group 3 (no surgery; $n=18$ ). Risk factors were evaluated with univariate and multivariate analysis. Results: Most patients who had NENCUP tumors (32 patients [84\%]) had World Health Organization (WHO) performance score of 0 or 1 , and most tumors (24 patients [63\%]) were well differentiated (WHO grade, G1 or G2; Ki-67 index, $\leq 20 \%$ ). Univariate analysis showed that greater survival was significantly associated with lower patient age, lower WHO performance score, lower WHO grade, lower number of metastatic sites, treatment with surgery, and no treatment with chemotherapy. Multivariate analysis showed that low WHO performance score and treatment with surgery were significant independent predictors of improved survival. Conclusion: In patients with NEN-CUP, surgical treatment is an independent predictor of better survival. Therefore, surgical treatment may be indicated in patients with good general health status and well differentiated NEN-CUP tumors. Keywords: outcome, nen-cup, surgery, survival. 


\section{2}

\section{Surgical Management of Carcinoid Valve Disease-Mechanical versus Tissue Valves}

Glasberg S. ${ }^{a}$, Atlan J. ${ }^{a}$, Korach A. ${ }^{a}$, Dabah A. ${ }^{a}$, Atlan K. ${ }^{a}$, Rudis E. a, Wald O. a, Elami A. ${ }^{a}$, Blau L. ${ }^{\text {, }}$ Gross D.J. ,

Reardon M.J. ${ }^{\text {, }}$ Shapira O.M. ${ }^{\text {a }}$

aHadassah-Hebrew University Medical Center, Jerusalem, Israel; ' The Methodist Debakey Heart and Vascular Institute, Huston, Tex., USA; 'The Methodist Debakey

Heart and Vascular Institute, Huston, Tex., USA

Introduction: The prosthetic valve of choice in patients with carcinoid valve disease (CVD) remains controversial due to the limited life expectancy of patients with advanced-stage neuroendocrine tumors (NETs) on one hand, and concerns regarding structural valve deterioration (SVD) on the other. Aim(s): We reviewed the records of 17 patients with right heart failure due to CVD. All patients received somatostatin analogues perioperatively. Data collected included baseline characteristics, procedural profiles and clinical outcomes. Materials and Methods: The primary NET site was ileum 12, lung 2 and stomach, colon, appendix-1 each. Preoperative urine levels of 5-HIAA $(61 \pm 36 \mathrm{mg} / 24 \mathrm{~h})$ and serum levels of chromogranin A $(2926 \pm 4057 \mathrm{ng} / \mathrm{ml})$ were 10 and 50 times greater than normal. Twenty two valves were implanted - tricuspid valve (TVR) -5 (tissue -4 , mechanical -1 ), TVR and pulmonary valve (PVR) -3 (tissue), PVR-1 (mechanical), TVR and mitral valve replacement (MVR) - 1 (mechanical). 30 -day mortality was $11 \%$. Mean follow up was $22 \pm 21$ months (range 1-81 months). Four patients developed SVD at $12,14,15$ and 20 months after surgery. Actuarial 4-year survival and freedom from SVD were $23 \pm 14 \%$ and $43 \pm 15 \%$, respectively. Results: The main advantage of tissue valve prostheses-avoiding lifelong, intense anticoagulation, might be offset by accelerated SVD. Conclusion: The use of mechanical valves should be considered in CVD patients with a large primary tumor mass, and persistent high levels of 5-HIAA despite therapy. Keywords: carcinoid valve disease.

\section{3 \\ Optimal Surgical Treatment of Pancreatic Neuroendocrine Tumors}

\author{
Jilesen A., Van Eijck C., Busch O., Van Gulik T., Gouma D., \\ Nieveen van Dijkum $E$.
}

Academic Medical Center, Amsterdam, The Netherlands

Introduction: For pancreatic neuroendocrine tumors (pNET), different types of pancreatic resections are available. Aim(s): The aim was to compare short and long-term outcomes after surgical resection in patients with pNET to select the optimal surgical treatment. Materials and Methods: All resected patients from two academic hospitals between 1992-2013 were included. Primary outcome was the short-term postoperative outcome. Secondary outcome was the long-term functional and oncological outcome. Two groups were compared; enucleation in the head vs. pancreatoduodenectomy
(PD) and enucleation in corpus/tail vs. distal pancreatectomy (DP). Risk factors for overall complications were identified in multivariable analyses. Results: 205 patients were included; enucleation in 60 , PD in 65, DP in 72. In-hospital mortality was $1.5 \%$, all after PD. The complication rate after enucleation from the pancreatic head (69\%) did not significantly differ compared to PD (80\%). The same yields for complication rates after enucleation and resection of the pancreatic tail (36 vs.58\%). Tumor located in the pancreatic head and $\mathrm{BMI} \geq 25 \mathrm{~kg} / \mathrm{m}^{2}$ were independent risk factors for complications after enucleation. During follow up, incidence of pancreatic insufficiency and recurrent disease was comparable between enucleation and DP. Conclusion: Complication rates were high but comparable for all procedures with a moderate risk for tumor recurrence after enucleation. Enucleation is therefore not a priori better and safer than standard resection. Keywords: enucleation, resection, pnet.

\section{4}

\section{Clinicopathological Characteristics and Long Term Survival Analysis of Pancreatic Neuroendocrine Incidentalomas: An Experience of 39 Cases from a Single Chinese Institute}

\author{
Leng J.H., Wei M., Li Y., Chen Z., Zheng O., Hao C. \\ Beijing Cancer Hospital, Beijing, China
}

Introduction: Pancreatic incidentaloma was found by coincidence without specific presentation like pancreatogenic abdominal pain, palpable mass, obstructive jaundice and endocrine symptoms. The characteristics and prognosis of the tumor were rarely discussed. $\operatorname{Aim}(\mathbf{s})$ : The study was to describe the clinicopathological characteristics and evaluate the prognosis of pancreatic neuroendocrine incidentalomas. Materials and Methods: The clinical data of 39 cases of pNETs who underwent surgical treatment between Aug 1996 and Sep 2011 were enrolled. The patients were divided into nonincidentaloma group and incidentaloma group. Kaplan-Meier method and $\log$ rank analysis was used to analyze the prognosis. Results: There were 15 patients in non-incidentaloma group, including abdomen or back pain in 8 cases, obstructive jaundice in 5 cases and hypoglycemia in 2 cases. The other 8 cases, presenting non-specific epigastric discomfort, together with the rest 16 cases without any symptom, were assigned to incidentaloma group. The mean size in incidentaloma group was $5.8 \mathrm{~cm}$, significantly larger than $4.2 \mathrm{~cm}$ in non-Incidentaloma group $(\mathrm{p}<0.05)$. The median survival duration of incidentaloma was 50.7 months, significantly shorter than 57.5 months in non-incidentaloma group $(\mathrm{p}<0.05)$. Conclusion: The pancreatic neuroendocrine incidentaloma has a relatively poor prognosis due to its non-specific presentation and delayed surgical intervention. More efforts should be paid to confirmation of the diagnosis and treatment for these patients. Keywords: incidentaloma, neuroendocrine, pancreas, survival. 
05

\section{Discrepancy between Radiologic and Pathologic Measurements in Pancreatic Neuroendocrine Tumors: A Retrospective Study on 199 Patients}

\author{
Malpaga A. ${ }^{a}$, Impellizzeri H. ${ }^{\text {a }}$, Miotto M. ${ }^{\text {, }}$ Cingarlini S. ${ }^{b}$, \\ Manfredi R. ', Capelli P. , Marchegiani G. ${ }^{\text {a }, ~ L a n d o n i ~ L . ~}{ }^{\text {, }}$ \\ Bassi C. ${ }^{\text {a }}$ Butturini $G$. ${ }^{\text {a }}$ \\ aPancreas Institute, Department of Surgery, Verona, Italy; \\ bPancreas Institute, Department of Oncology, Verona, \\ Italy; 'Pancreas Institute, Department of Radiology, \\ Verona, Italy; dPancreas Institute, Department of \\ Pathology, Verona, Italy
}

Introduction: Preoperative radiologic size of pancreatic neuroendocrine tumors (PNET) is a crucial parameter in selection of appropriate treatment especially for incidentally discovered lesions $(<20 \mathrm{~mm})$. The current guideline bases the therapeutic recommendations on the pathologic size. $\operatorname{Aim}(\mathbf{s})$ : Evaluate the discrepancy between radiologic size (RS) and pathologic size (PS) of PNET. Materials and Methods: Patients resected for PNET between January 2004 and November 2014 at our Institution were retrieved from a electronic database and analyzed retrospectively. We excluded functioning and cystic PNET and R2 resections. RS is defined as the mean of the largest diameters measured on CT, EUS and MRI, and PS as the largest diameter of the tumor on the histological report of the surgical specimen. Results: Study populations consisted of 199 patients (97 females/102 males), with a median age of 56 years (23-83). Overall the RS $(32.7 \pm 22.8 \mathrm{~mm})$ and PS $(31.1 \pm 23.4 \mathrm{~mm})$ did not significantly differ $(p=0.477)$. In the subgroup analysis, the size difference was statistically significant for tumor size of less then $30 \mathrm{~mm}(\mathrm{p}=0.0006)$. In this last group $(\mathrm{n}=133)$, RS overestimated PS in $66 \%(n=88)$ and RS underestimated PS in $21 \%(n=28)$. Furthermore, we selected patients with PS $<20 \mathrm{~mm}$ but with a RS $>20 \mathrm{~mm}(\mathrm{n}=23)$, and 13 of these were incidentalomas. Conclusion: Radiologic preoperative imaging overestimates the size of tumor especially in PNET $<30 \mathrm{~mm}$. The overestimation could be of paramount importance in the management of incidentally diagnosed small $(<20 \mathrm{~mm})$ PNET. Keywords: size, net.
06

\section{Long-Term Follow-Up of Resected Small $(<2 \mathrm{~cm})$ Asymptomatic Sporadic Non-Functioning Pancreatic Neuroendocrine Tumors: Correlation between Type of Resection, Histopathologic Features, and Outcome}

\author{
Malpaga A. ${ }^{a}$, Impellizzeri H. ${ }^{a}$, Ficial M. ${ }^{\text {, }}$ Miotto M. ${ }^{\text {a }}$ \\ Boninsegna L. ${ }^{c}$, Cingarlini S. ${ }^{\mathrm{d}}$, Capelli P. ${ }^{\mathrm{b}}$, Malleo G. , \\ Landoni L. ${ }^{a}$, Scarpa A. ${ }^{\text {b }}$, Bassi C. ${ }^{a}$, Butturini G. ${ }^{\mathrm{e}}$ \\ aPancreas Institute, Department of Surgery, Verona, Italy; \\ bPancreas Institute, Department of Pathology, Verona, \\ Italy; 'Department of General Surgery, Negrar, Italy; \\ dPancreas Institute, Department of Oncology, Verona, \\ Italy; ePancreas Institute, Department of Surgery, Verona, \\ Italy
}

Introduction: The surgical management of small, incidentally found sporadic non-functioning pancreatic neuroendocrine tumors (NF-PNET) is controversial. Aim(s): Evaluate the long-term outcome after surgical resection (SR) of sporadic, asymptomatic NF-PNET $<2 \mathrm{~cm}$, stratifying the results by SR type and histopathologic features. Materials and Methods: Patients resected between June 1997 and November 2009 at our Institution (minimum followup of 60 months) were retrieved from an electronic database and analyzed retrospectively. Results: Study population consisted of 56 patients $(31 \mathrm{~F} / 25 \mathrm{M})$, with a median age of 57 years (range 23-83). 37 patients underwent parenchyma-sparing SR (18 enucleations, 17 middle pancreatectomies, 2 spleen-preserving distal pancreatectomies), while 19 patients underwent formal SR ( 9 pancreaticoduodenectomies, 10 distal pancreatectomies with splenectomy). Mean lymph node yielding was $1.32(0-14)$ in atypical SR and 20.5 (9-41) in formal SR. The median tumor size was $14 \mathrm{~mm}$. Lymph node metastases were found in 3 patients. 54 tumors were G1, 2 was G2, none was G3. The final pathologic report showed microscopic local invasion (perineural, lymph vascular or fat invasion) in 9 patients. At a median follow-up of 80 months (60-193), 5 patients died of other causes, 5 were lost to follow-up and 46 patients did not develop recurrence. Conclusion: Long-term outcome after SR of asymptomatic sporadic NF-pNET $<2 \mathrm{~cm}$ is excellent. Microscopic local invasion and lymph node metastases did not impact on the natural history of the disease. Keywords: small.

07

\section{Pathological Factors Predicting Recurrence of the Non-Functioning Pancreatic NET after Resection}

Masui T., Sato A., Takaori K., Uemoto S.

Kyoto University, Kyoto, Japan

Introduction: The usefulness of the WHO 2010 classification for surgeons has not been elucidated, since the criteria is defined by cell growth with Ki67 and may not represent invasiveness of the tumor 
as well as the recurrence type (liver metastasis or lymph node metastasis). Aim(s): The purpose of the study is to elucidate if the WHO 2010 classification is appropriate for the estimation of the tumor malignancy and is applicable to the estimation of recurrence after tumor resection especially for nonfunctioning NET. Materials and Methods: From January 2000 to June 2013, we had 98 pancreatic NET patients who underwent resection without distant metastasis. We analyzed 41 non-functioning PNET patients for WHO 2010 classification and other pathological factors with survivals and recurrent locus. Results: There are 26 G1 NET patients, 13 G2 NET patients and 2 NEC patients. (1) WHO2010 predicted disease free survival (DFS) $(p=0.0041)$. (2) Pathological lymphatic invasion(ly), vein invasion (v) and lymphnode metastasis affected disease free survival $(\mathrm{p}=0.0003$, $\mathrm{p}=0.0001$ ) but tumor peripheral invasion did not. (3) Ly, v, peripheral invasion did not correlate with recurrence type. (4) WHO2010 status correlated with invasiveness such as Ly, v. (5) Lymphnode metastasis was an independent factor for recurrence $(p=0.0025)$. Conclusion: WHO2010 criteria represented tumor invasiveness. Of note, nodal status predicted DFS independently, suggesting that the examination of the nodal metastasis is highly recommended even when enucleation. Keywords: who2010, invasiveness, resection.

\section{8 \\ Aggressive Multi-Modality Treatment of Pancreatic Neuroendocrine Tumors (pNETs): Outcomes in Patients with Advanced Disease}

Mayo D., Armstrong T., Cave J., Nolan L., Steadman B., Connor H., Sundram F., Abu-Hilal M., Takhar A., Pearce N.

Southampton General Hospital, Southampton, UK

Introduction: pNETs carry the worst prognosis of all NETs, particularly when hepatic metastasis are present. We try to actively treat all patients referred. Aim(s): We aimed to determine survival at 1,3 \& 5 years for pNETs treated with modern aggressive multimodal management and compare outcomes to previously published data. Materials and Methods: Data was analysed from a single centre, prospective database. 95 of 396 NET patients were identified as pNETs. 3 patients had incomplete records and were excluded. Outcomes were analysed using Kaplan-Meier, with death as the endpoint. Results: 92 patients with a median follow up 38 months. 35 patients stage 1-3 disease (median follow up 42 months) and 2 deaths. Kaplan-Meier survival curve showed $97 \%$ at 1 year and $93 \%$ at 3 \& 5 years. 57 patients had stage 4 disease. Pattern of disease was liver metastasis $n=42$, liver and distant metastasis $n=13$, distant metastasis only $n=2.3$ patients were palliative care, one refused treatment but all included in statistical analysis. 53 stage 4 patients had uni/multimodal treatment comprising; somatostatin analogues $(n=34)$, surgery $(n=35)$, TACE $(n=21)$, PRRT $(n=4)$ and chemotherapy $(n=$ $40)$. For stage 4 disease: Mean survival $=83.7$ months. Survival rate estimates were, $1 \mathrm{yr}=91 \%, 3 \mathrm{yr}=74 \%, 5 \mathrm{yr}=60 \%$. Conclusion: Aggressive multimodal treatment is likely to confer a survival advantage in stage 4 pNET patients compared to historical studies, which variably report 5 year survival at $13.7-36 \%$ at 5 years in patients with stage 4 disease. Keywords: pnet, multimodal, neuroendocrine.
09

\section{Experience of Two European Centers on Central Pancreatectomy for NeuroEndocrine Tumors of the Pancreas}

\author{
Milanetto A.C. ${ }^{\text {a }}$ Pasquali C. ${ }^{a}$, Lico V.a , Büchler M.W. ${ }^{\text {, }}$ \\ Hackert T. ${ }^{\text {b }}$ \\ aPancreatic and Digestive Endocrine Surgical Unit, \\ Padua, Italy; ' Klinik für Allgemein-, Viszeral-und \\ Transplantationschirurgie, Heidelberg, Germany
}

Introduction: Central pancreatectomy (CP) is a parenchimasparing operation that enables the removal of benign or low-grade malignant lesions from the neck of the pancreas. Aim(s): We reported our experience of two high-volume pancreatic centers (Padua-I, and Heidelberg-D), regarding outcome and follow up (FU) of 26 neuroendocrine tumors (NET) patients submitted to CP in the last 15 years. Materials and Methods: Data of patients operated from 2000 to 2014 were retrieved retrospectively, and evaluated in terms of complications, and post-operative pancreatic function. FU to July 2014. Results: From March 2000 to January 2014 in our Centers, out of 129 patients submitted to CP, 26 had a NET. Average 52 years (range 37-74), $9 \mathrm{M} / 17 \mathrm{~F}$. Median tumor size $1.3 \mathrm{~cm}$ (range 0.7-4.0). Histology: 2/26 malignant NET (lymph node metastasis), 24 were G1 and 2 G2 (Ki67 <4\%), 21 were nonfunctioning (NF) and 5 insulinoma. 16/26 patients had a post-operative pancreatic fistula (POPF), half were grade $\mathrm{C}$, treated by interventional radiology. Median hospital stay 15.5 days (range 8-63). 2/5 patients with a post-operative pancreatic hemorrhage ( $\mathrm{PPH}$ ) needed surgery; one patient died. About $8 \%$ of patients had new onset or worsening mellitus diabetes; no exocrine insufficiency. After a median FU of 88 months (range 6-169) there was no evidence of disease recurrence. Conclusion: $\mathrm{CP}$ is a safe alternative to the standard pancreatic operations for NETs; Morbidity rate is superior but CP preserves better endocrine and exocrine pancreatic function. Keywords: net, central pancreatectomy. 


\section{0}

Pancreatic Neuroendocrine Incidentalomas: When Is Surgery in the Patient's Best Interests?

\author{
Mills L. ${ }^{a}$, Ramage J. ${ }^{\mathrm{b}}$, Prachalias A. c, Srinivasan P., \\ Menon K. c, Quaglia A. ', Vivian G. a, Heaton N. ', Sarker D. a, \\ Ross P. ${ }^{a}$ Basuroy R. ${ }^{\text {a }}$ Srirajaskanthan R. ${ }^{\text {e }}$ \\ aENETS Centre of Excellence, Institute of Liver Studies, \\ King's College Hospital, London, UK; b Department of \\ Gastroenterology, Hampshire Hospitals NHS Trust, \\ Basingstoke, UK; ' Hepatopancreatobiliary Surgery, \\ Institute of Liver Studies, King's College Hospital, \\ London, UK; d Department of Histopathology, Institute \\ of Liver Studies, King's College Hospital, London, UK; \\ eDepartment of Gastroenterology, University Hospital \\ Lewisham, London, UK
}

Introduction: Small PNETs, many of which are diagnosed incidentally, pose a management dilemma. Whilst resection offers curative outcomes, the risks of surgery have led many to adopt surveillance strategies. $2 \mathrm{~cm}$ is often used as a threshold for malignancy, but reports suggest that this value may not be valid. $\mathbf{A i m}(\mathbf{s})$ : To investigate the predictors of malignancy in small PNETs. Materials and Methods: The notes of 233 patients with PNETs of all stages were reviewed for pathology reports. Tumours were defined as malignant if displaying local invasion, vascular invasion, nodal involvement or distant metastasis (metachronous or synchronous). Predictors of malignancy were considered using logistic regression. Results: Malignant characteristics were shown to correlate with tumour diameter $(\mathrm{r} 2>0.8)$. However, of 68 tumours $<2 \mathrm{~cm}, 21$ (31\%) developed local or vascular invasion, 18 (28\%) nodal metastasis and $16(24 \%)$ distant metastasis. 164 tumours displayed malignant characteristics, 64 had no evidence of malignancy and data was unavailable for 5. Significant univariate predictors of malignancy included diameter (mean $21 \mathrm{~mm} \mathrm{vs.} 47 \mathrm{~mm}$, $\mathrm{p}<0.001)$, symptomatic presentation vs. asymptomatic $(\mathrm{p}<0.001)$, familial syndromes $(\mathrm{p}<0.022)$ and elevated serum CgA $(p<0.001)$. Multivariate analysis showed that diameter, symptomatic presentation and elevated $\mathrm{CgA}$ were independent predictors of malignancy $(p<0.001, p=0.001, p=0.019)$. Conclusion: $40 \%$ of PNETs $<2 \mathrm{~cm}$ display malignant characteristics. Diameter, $\mathrm{CgA}$ and symptoms can be used to predict the likelihood of malignancy. Keywords: pnet.

\section{1}

\section{Long-Term Outcomes of Surgical Management of Pancreatic Neuroendocrine Tumors with Synchronous Liver Metastases}

Partelli S. ${ }^{a}$, Inama M. ${ }^{\text {, }}$ Rinke A. ${ }^{c}$, Begum N. ${ }^{d}$, Valente R. ${ }^{b}$, Fendrich V. ${ }^{\mathrm{c}}$, Tamburrino D. ${ }^{\mathrm{b}}$, Keck T. ${ }^{\mathrm{d}}$, Caplin M. ${ }^{\mathrm{b}}$,

Bartsch D. ${ }^{c}$, Fusai G. ${ }^{\text {b }}$ Falconi M. ${ }^{\mathrm{a}}$

a University Hospital Ancona, Ancona, Italy; ${ }^{\text {bRoyal Free }}$ Hospital, London, UK; ' University Hospital Marburg, Marburg, Germany; dUniversity of Lubeck, Lubeck, Germany

Introduction: The value of surgical resection in the management of pancreatic neuroendocrine tumor (PNET) with liver metastases (LM) is still debated. Aim(s): The aim of the study was to evaluate the outcomes of surgery of PNET with LM. Materials and Methods: Patients with PNET with LM (2000-2011) from 4 Institutions were included. Patients were divided into 3 groups (curative resection, palliative resection, and no resection). Results: 169 patients were included. Nineteen patients (11\%) underwent curative resection, 74 (44\%) underwent palliative resection, and 76 (45\%) underwent conservative treatment. The median overall survival (OS) from was 73 months. Patients who underwent curative resection had a significantly better median OS from diagnosis compared with those who underwent palliative resection and those who were not resected (97 versus 89 vs. 36 months, $\mathrm{P}=0.0001$ ). The median OS from diagnosis in those patients who underwent radical or palliative resection was 96 months. On multivariate analysis, factors associated with OS from diagnosis were bilobar metastases and curative resection in a first model. On a second model, curative or palliative surgery was a predictor of OS. Among 93 patients who underwent surgery, the presence of PNEC-G3 was the only factor independently associated with a poorer survival after surgery (median OS: 35 months versus 97 months, $\mathrm{P}<0.0001$ ). Conclusion: Patients with LM from PNET benefit from surgery although it should be reserved to well- or moderately differentiated forms. Keywords: surgery, metastases, net, survival.

\section{2 \\ Initial Experience with Robot-Assisted Distal Pancreatectomy for Pancreatic Neuroendocrine Neoplasms

\author{
Partelli S. ${ }^{a}$, Cirocchi R. ${ }^{b}$, Crippa S. ${ }^{a}$, Desiderio J. ${ }^{\text {, }}$ \\ Belfiori G. ${ }^{a}$, Parisi A. ${ }^{\text {b }}$, Falconi M. ${ }^{\text {a }}$ \\ ${ }^{a}$ Ancona University Hospital, Ancona, Italy; ${ }^{b}$ Hospital of \\ Terni, Terni, Italy
}

Introduction: Robot-assisted distal pancreatectomy (RADP) for pancreatic neuroendocrine neoplasms (pNEN) has not been investigated so far. $\mathbf{A i m}(\mathbf{s})$ : To compare short-term outcomes of RADP with open distal pancreatectomy (ODP) for pNEN. Materials and Methods: This was a retrospective case-control study of 10 patients undergoing RADP using the da Vinci ${ }^{\circledR}$ Surgical System (Intuitive 
Surgical, Inc., Sunnyvale; Calif., USA) for PNEN at two institutions (Ospedali Riuniti Ancona and Ospedale S.Maria Terni) between January 2012 and June 2014. Patients who underwent RADP were compared with a case-matched group of 20 patients who underwent ODP. Results: Two groups were similar as regards of age, BMI, ASA score, tumor size and tumor grading. The median of examined lymph nodes in the ODP group was 14.5 (range 5-32) compared with 11.5 $(6-20)$ in the RADP group $(p=0.091)$. Overall mortality was nil. Robot-assisted procedures were significantly longer compared to ODP (257.5 minutes versus 200 minutes, $\mathrm{p}=0.030)$. The median estimated blood loss was lower during RADP $(115 \mathrm{ml}$ versus $350 \mathrm{ml}, \mathrm{p}=$ 0.030). Rate of postoperative complications did not differ between the two types of procedures. Patients in the RADP group had a significantly shorter length of hospital stay (LOS) compared with the ODP group ( 5.5 days versus 10.5 days, $p=0.001)$. Conclusion: Although the operative time is significantly longer, RADP for pNEN seems to guarantee a lower blood loss and a shorter LOS. Keywords: robot-assisted pancreatectomy, pancreatic neuroendocrine neoplasm, surgery.

\section{3 \\ How Manage Multiple Small Bowel Neuroendocrine Tumors?}

Pasquer A., Walter T., Hervieu V., Forestier J., Scoazec J.Y., Lombard-Bohas C., Poncet G.

Hospices Civils de Lyon, Hopital E Herriot, Lyon, France

Introduction: Multiple small bowel neuroendocrine tumors (SB-NET) represent $30 \%$ of SB-NET. Aim(s): This retrospective study aimed to compare multiple versus unifocal SB-NET and to describe surgical management of SB-NET. Materials and Methods: All consecutives patients with SB-NET who underwent the resection of at least one primary SB-NET, between 01/01/2000 and 01/01/2013 were analyzed. All morphological workup done before surgery, histological classifications and the lymph node (LN) ratio (LN involved/removed) were recorded. Results: 107 patients underwent a resection of SB-NET: $70 \%$ had metastatic disease, $43 \%$ had carcinoid syndrome, 90\% had lymph nodes (LNs) involved. Median number of nodes removed was 12; the LNs ratio (LNs involved of LNs removed) was 0.3 . Thirty-five (33\%) patients had multiple SB-NET [mean of 3.1 (1-44) tumors]. All preoperative imaging and surgeon missed $61 \%$ and $33 \%$ of SB-NET. Characteristics between multiple and unifocal SB-NET are similar except occurrence of higher carcinomatosis, serum Chromogranin A levels, and angioinvasion in multiple SB-NET. $12 \%$ of patients had local lymph nodes recurrence. Local recurrence free survival on $\mathrm{LN}$ at 5 years was $85 \%$. Median overall survival was 128 (91-165) months. After multivariate analysis, high $\mathrm{CgA}$ levels and histological carcinomatosis were significantly associated with worse overall survival. Conclusion: Palpation of the whole SB detects more multiple NET than preoperative imaging. Extensive LN resection prevents SB complications. Keywords: small bowel, surgery, prognosis, multiple.

\section{4}

100 Cases of Pancreatic Resections for Pancreatic Neuroendocrine Tumors. The Royal Free Hospital Experience

\author{
Tamburrino D. a, Valente R. a, Inama M. a, Toumpanakis $C$. , \\ Thirlwell C. ', Luong T. , Davidson B. a, Imber C. ,

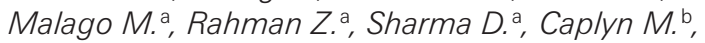 \\ Fusai G.K. ${ }^{a}$ \\ aRoyal Free Hospital NHS Foundation Trust, HPB and Liver \\ Transplant Surgery, London, UK; ' Royal Free Hospital \\ NHS Foundation Trust, Neuroendocrine Tumour Unit, \\ ENETS Centre of Excellence, London, UK; ' Department \\ of Oncology UCL of London, London, UK; ${ }^{d}$ Royal Free \\ Hospital NHS Foundation Trust, Department of Patology, \\ London, UK
}

Introduction: Pancreatic neuroendocrine tumours (pNETs) are rare neoplasms of variable grade of malignancy. Surgery is the only curative treatment. $\mathbf{A i m}(\mathbf{s})$ : To analyse the morbidity and mortality rate, the disease free survival (DFS) and overall survival (OS) following resection for $\mathrm{p}$-NETs in a single high-volume centre. Materials and Methods: From April 1998 until May 2014, 100 patients underwent pancreatic resection for p-NET at Royal Free Hospital. Morbidity was defined based on Dindo-Clavien postoperative classification, mortality rate was defined as related to surgery until 90th post-operative day. Results: One-hundred cases were included. Tumours were non-functioning in $79 \%$ of the patients. Forty-two $(42 \%)$ of patients underwent pancreaticoduodenectomy (PD). Thirty one were ENETS stage I, 29 stage II, 24 stage III, 16 stage IV. No perioperative mortality was recorded. Pancreatic fistula occurred in 24 (25\%) patients, only 2 patients (2\%) had grade C fistula. ClavienDindo (CD) postoperative morbidity was recorded in $40(40 \%)$, among these 22 patients (55\%) had CD grade 2. OS was $94.1 \%$ at 5 -years. Median DFS was $38.1 \%$, and $23.8 \%$ at 5 and 10 years respectively. Conclusion: In our experience, primary surgical resection for p-NETs had no postoperative mortality. Morbidity rate is comparable to largest series reported in literature. Pancreatic resections for NET disease should be preformed in a high volume multidisciplinary centre to achieving a good postoperative outcome and prolonged overall survival. Keywords: pancreatic neuroendocrine tumor, surgery.

015

\section{Development of Effective Prophylaxis Against Intraoperative Carcinoid Crisis}

Woltering E. ${ }^{a}$, Diebold A. ${ }^{a}$, Stevens M. ${ }^{\text {a }}$ Wang Y.Z. ${ }^{\text {, }}$ Boudreaux J. ${ }^{a}$, Mamikunian G. ${ }^{\text {, }}$ Riopelle J. ${ }^{a}$, Kaye A. ${ }^{a}$

aLSUHSC-NO, New Orleans, USA; 'InterScience Institute, Inglewood, USA

Introduction: Patients with foregut and midgut neuroendocrine tumors (NETs) can experience life-threatening carcinoid crises during anesthesia. The prophylactic use of a pre-, intra- and post-operative high dose continuous octreotide infusion was evaluated for its ability 
to prevent carcinoid crises during cytoreductive surgeries. Aim(s): To develop an effective anesthesia protocol to prevent intraoperative carcinoid crises. Materials and Methods: Anesthesia and surgical records of 188 patients who underwent a total of 228 cytoreductive surgeries for stage IV, small bowel NETs were reviewed. All patients received a 500 microgram/hour infusion of octreotide pre-, intra- and post-operatively. Patients that experienced intraoperative hyper- or hypotension, profound tachycardia, or a 'crisis' according to the operative note were also reviewed. Results: Two-hundred and seventeen $(217 / 228,95 \%)$ patients had normal anesthesia courses. The charts of 11 patients were further investigated for a potential intraoperative crisis using the listed criteria. Following critical review by surgeon and anesthesiologist, six patients were determined to have had an intraoperative crisis. The final incidence of intraoperative crisis using a continuous octreotide infusion was 3\% (6/228). Conclusion: A continuous preoperative, intraoperative and postoperative high-dose octreotide infusion prevents carcinoid crisis. We believe that the low cost and excellent safety profile of octreotide warrants the use of this therapy during major surgical procedures. Keywords: carcinoid crisis.

\section{6 \\ Liver Transplantation for Metastatic Hepatic Neuroendocrine Tumors of Unknown Origin

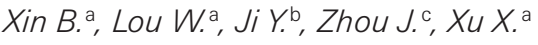 \\ aDepartment of Pancreatic Surgery, Zhongshan Hospital, Fudan University, Shanghai, China; 'Department of \\ Pathology, Zhongshan Hospital, Fudan University, Shanghai, China; ' Liver Cancer Institute, Zhongshan \\ Hospital, Fudan University, Shanghai, China}

Introduction: Liver transplantation can be implemented on patients with diffuse hepatic tumors, but for hepatic NETs its role and value are still unclear. $\mathbf{A i m}(\mathbf{s})$ : To operate liver transplantation for appropriate patients with NETs and observe its efficacy. Materials and Methods: The clinical and pathological datas of 3 patients who were performed LT in our hospital from Dec 2007 to Jul 2014 were retrospectively analysed. Results: All of them were male and the age range was 56 59 years old. No extrahepatic lesions were found and the primary tumors were unknown in the preoperation, but after surgery one case was confirmed duodenum origin. Up to now, one patient was dead, whereas the other two survived well. The dead underwent combined liver-kidney transplantation (no ki-67 data) without any obvious complications. 32-month later, tumor metastasized to the lumbar (PFS = 32-month) and he died at 58-month after CLKT (OS $=58$-month). Another one $(\mathrm{Ki}-67=20 \%)$ readmitted after a month due to fever. He recovered himself through anti-infection treatment, and now he has survived 23-month. The third one once underwent the left liver resection $(\mathrm{Ki}-67=20 \%)$, whereas 18 -month later, he was discovered diffuse lesion in his right liver (Ki-67 soared to $40 \%$ ). He took the examination of octrotide scanning post-LT, then the primary tumor of duodenum was discovered. To date, he lives well for 3-month. Conclusion: For selected patients of NETs, LT can prolong their survival time and the efficacy is good. Keywords: hepatic nets, lt.

\section{Non Digestive NETs (Bronchial, MTC, Pheochromocytoma)}

\section{P1 \\ A National Pathology Registration Analysis of Applied Terminology in Pulmonary Neuroendocrine Tumors/Carcinomas: Is the WHO 2004 Classification in Line with Clinical Practice?}

Derks J. ${ }^{\text {, }}$ Dingemans A.M.C. a, Van Suylen R.J. ${ }^{\text {, }}$ Thunnissen E. ', Den Bakker M.A. ${ }^{d}$, PALGA: Dutch Pathology Registration ${ }^{e}$, Speel E.J. ${ }^{\dagger}$

aDepartment of Pulmonary Diseases, GROW School for Oncology \& Developmental Biology, Maastricht University Medical Centre, Maastricht, The Netherlands; bDepartment of Pathology, Jeroen Bosch Hospital, s'Hertogenbosch, The Netherlands; 'Department of Pathology, VU Medical Center, Amsterdam, The Netherlands; dDepartment of Pathology, Maasstad Hospital, Rotterdam, The Netherlands; 'PALGA, The Nationwide Network and Registry of Histo- and Cytopathology in The Netherlands, Houten, The Netherlands; 'Department of Pathology, GROW School for Oncology \& Developmental Biology, Maastricht University Medical Centre, Maastricht, The Netherlands

Introduction: Nomenclature of the WHO 2004 should be used for pulmonary neuro-endocrine tumors (NET) and carcinomas (NEC) classification. Aim(s): To analyze if current practice is in line with the WHO 2004 terminology (TI), conclusions (CN) of pathology reports of NET/NEC diagnosed in the Netherlands were analyzed. Materials and Methods: CN of NET/NEC diagnosed from 2003-2012 were selected from the Dutch Pathology Registry through 4 queries of SNOMED codes (e.g. NET grade I, M8242_) and 'free text' (e.g. carcinoma + endocrine). All CN were screened; $\mathrm{CN}$ describing non-lung, non-NET/NEC, small cell cancer, or autopsy were excluded. CN were clustered into biopsy/cytology $(\mathrm{Bx})$ or resection $(\mathrm{Rx})$ and compared with current TI. Results: A total of $7989 \mathrm{CN}$ were retrieved of which $4092 \mathrm{CN}$ of 2656 patients ( $36 \%$ had $\geq 2 \mathrm{CN}$, range $1-7$ ) were eligible for analysis. Of these $\mathrm{CN}, 1086 / 975(\mathrm{Bx} / \mathrm{Rx})$ reported NET, 1111/677 (combined) NEC and 222/23 were inconclusive. CN in $10 \%$ of NET and $22 \%$ of NEC, mostly established on Bx (74\%), did not comply with current TI. The diagnosis of typical/atypical carcinoid and large cell neuroendocrine carcinoma was established on $\mathrm{Bx}$ in $40 \%$ (448 $\mathrm{CN})$ and $58 \%(632 \mathrm{CN})$, respectively. Conclusion: A discrepancy exists between clinical practice and the advised WHO 2004 TI of pathology report CN describing NET (10\%) and NEC (22\%). This occurred more often on Bx than on Rx specimens. Whether this is due to personnel preferences or to difficulties with fitting the current classification on small samples, remains to be examined. Keywords: net, diagnosis. 


\section{P2}

\section{Clinical Characteristics and Overall Survival of Large Cell Neuroendocrine Carcinoma (LCNEC) Compared to Other Subtypes of Lung Cancer: Results of a Population-Based Registry}

Derks J. ${ }^{a}$, Hendriks L.E. ${ }^{\text {a }}$ Damhuis R.A. ${ }^{\text {b }}$, Speel E.J. ${ }^{c}$, Dingemans A.M.C. ${ }^{\text {a }}$

aDepartment of Pulmonary Diseases, GROW school for Oncology \& Developmental Biology, Maastricht University Medical Centre, Maastricht, The Netherlands; bDepartment of Registry and Research, Comprehensive Cancer Centre, Rotterdam, The Netherlands: 'Department of Pathology, GROW School for Oncology \& Developmental Biology, Maastricht University Medical Centre, Maastricht, The Netherlands

Introduction: LCNEC is a rare non-small cell lung cancer subtype and clinical behavior is unclear. $\mathbf{A i m}(\mathbf{s})$ : To analyze incidence, patient (pt) characteristics, metastatic pattern and overall survival (OS) of LCNEC and compare data with small-cell (SCLC), squamous cell ( $\mathrm{SqCC}$ ) and adenocarcinomas ( $\mathrm{AdC})$. Materials and Methods: Histologically confirmed LCNEC, SCLC, SqCC and AdC cases diagnosed between 2003-2012 were selected from the Netherlands Cancer Registry. Cohorts of stage I-II, III and IV disease were compared for pt characteristics, metastasis at diagnosis $(\geq 2006)$ and OS. Multivariate Cox regression was performed; if non-proportional, time dependent Hazard ratios (HR) were reported. Results: Data from 952 LCNEC, 11,844 SCLC, 19,633 SqCC and 24,253 AdC were extracted. Incidence of LCNEC increased with $255 \%$ from 56 to 143 pts (2003-2012), particularly in stage IV. LCNEC pts had metastasis in liver $47 \%$, bone $32 \%$, brain $23 \%$, adrenal gland $19 \%$, extra thoracic lymph node $16 \%$, lung $14 \%$ and pleura $7 \%$. LCNEC significantly differed with SCLC in 2/7 involved organs, whereas SqCC and AdC differed in 5/7 organs. Median OS of stage I-II, III and IV LCNEC was 32.4 (22.0-42.9), $12.6(10.3-15.0)$ and 4.0 (3.5-4.6) months, respectively. Stage IV OS ( $\leq 3 \&>3$ months) resembled SCLC (HR $0.92 \&$ HR 0.92, p $>0.05)$ yet was worse than $\mathrm{SqCC}(\mathrm{HR}=0.74 \& \mathrm{HR} 0.84$, $\mathrm{p}<0.01$ ) and $\mathrm{AdC}(\mathrm{HR} 0.75 \& \mathrm{HR} 0.78, \mathrm{p}<0.01$ ). Conclusion: Over 10 years' time LCNEC incidence increased 2.5 fold. In stage IV LCNEC the metastatic pattern and OS resembled SCLC more than SqCC and AdC. Keywords: lcnec, lung.

\section{P3}

\section{Atypical Bronchial Carcinoid: Different Therapeutical Approachs}

\author{
Geraldo Roig L. \\ Universität Innsbruck, Innsbruck, Austria
}

Introduction: Choosing an adequate treatment for atypical bronchial carcinoid can improve quality of life and survival. Aim(s): We present 2 examples of different therapeutic decisions in metastatic disease. Materials and Methods: 74 years old patient was diagnosed with a right pulmonar nodule as well as ipsilateral hiliar lymphadenopathy, liver and disseminated bone metastases. Histology demostrated a Ki67:12\%. Two years after diagnosis the patient underwent chemotherapy with Everolimus. Afterwards, Peptide Receptor Radionuclide Therapy (PRRT) with 90Yttrium-DOTATOC was started, achieving a disease stabilisation. Therapy control was performed with 68GaDOTATOC PET-CT showing no significant changes. An additional 18FFDG PET was negative. At present the patient is treated by octeotride therapy. 20 years old patient with atypical bronchial carcinoid in the left lung showed a Ki67:3\%. Initially he underwent surgery with lobectomy and local lymphadenectomy. Two years later 68GaDOTATOC PET-CT controls demonstrated diseminated liver metastases. A stabilisation was finally achieved with octreotide. Results: In both cases a systemic treatment with somatostatin analogs was well tolerated, reduced clinical symptoms and stabilized the disease. Conclusion: In the decision for the therapeutic use of radiolabeled somatostatin analogs also patient age and renal function was taken into account. Moreover, the tumor proliferation played a role in treatment decision. Keywords: 68 gadotatoc pet-ct, neuroendocrine tumor, atypical bronchial carcinoid, octreotide, prrt.

P4

Everolimus in Patients (pts) with Advanced Lung Carcinoids: Evaluation of Median Progression Free Survival (PFS) and Median Time to First Dose Decrease or Discontinuation (DDD)
Gianoncelli L. ${ }^{a}$, Guigay J. ${ }^{\text {, }}$ Caramella C. ${ }^{c}$, Borget I. , Mir O. ${ }^{e}$, Del Prete M. ${ }^{\dagger}$, Ducreux M. ${ }^{9}$, Santoro A. ${ }^{\text {, }}$ Finocchiaro G. ${ }^{a}$, Scoazec J.Y. ${ }^{\mathrm{h}}$, Baudin E. i, Planchard D. ${ }^{\mathrm{j}}$
aDepartment of Medical Oncology and Hematology, Humanitas Clinical and Research Center, Rozzano, Milan, Italy; ${ }^{b}$ Centre Antoine-Lacassagne, Nice, France; 'Department of Diagnostic Imaging, Gustave Roussy, Villejuif, France; dDepartment of Biostatistics and Epidemiology, Gustave Roussy, Villejuif, France; eDepartment of Clinical Pharmacology, Gustave Roussy, Villejuif, France; fDepartment of Clinical Medicine and Surgery, Section of Endocrinology, Federico II University, Naples, Italy; 9Department of Medicine, Gustave Roussy, Villejuif, France; ' Department of Pathology, Gustave Roussy, Villejuif, France; 'Department of Nuclear Medicine and Endocrine Tumors, Gustave Roussy, Villejuif, France; jDepartment of Medical Oncology, Thoracic Group,
Gustave Roussy, Villejuif, France

Introduction: Treatment of well-differentiated (WD) lung neuroendocrine tumors (NETs) is challenging. Everolimus (mTOR inhibitor) has shown encouraging results in digestive NETs but data about its activity on lung carcinoid are sparse. Aim(s): The aim of our study was to assess antitumoral efficacy and tolerability of everolimus in lung NETs. Materials and Methods: Consecutive pts with progressive advanced WD lung NETs treated with everolimus at Gustave Roussy between Sep 2007 and Feb 2014 were analyzed. Treatment regimen with oral everolimus was $10 \mathrm{mg}$ daily. Primary end-points 
were median PFS and median time to first DDD. Secondary objective were overall survival (OS), best response (RECIST v1.1) and safety. Results: 35 pts were enrolled, there were 18 male and 17 female, the mean age was 60 years (range 23-86). Most pts (63\%) had a typical carcinoid. With a median follow-up of $42 \mathrm{~m}$, the median PFS was $10.6 \mathrm{~m}$ (95\% CI 7.3-12.6). Stable disease was observed as best response in 33 pts $(94 \%)$. Median time to first drug DDD for toxicity was $4.9 \mathrm{~m}$ (95\% CI 2.2-7.8). Drug related adverse events lead to drug withdrawal in $34 \%$ of pts. The most common AE resulting in treatment withdrawal were asthenia (14.3\%), non-infectious pneumonitis (8.5\%) and stomatitis (5.7\%). Median OS was $30.8 \mathrm{~m}$ (95\% CI 21.4-52.4). Conclusion: A median PFS of $10.6 \mathrm{~m}$ with a median time to first DDD of $4.9 \mathrm{~m}$ were observed, suggesting that in lung NETs everolimus dose drug intensity might be suboptimal leading to insufficient clinical activity. Keywords: lung carcinoid, everolimus, mtor inhibitor.

\section{P5 \\ Clinical and Pathologic Characteristics of Small Cell Neuroendocrine Carcinoma of the Bladder}

\author{
Perez-Casanova L., Taco M.D.R., Vidal A., Goma M., \\ Condom E. \\ Hospital Universitari de Bellvitge, Barcelona, Spain
}

Introduction: Small cell carcinoma of the bladder (SCC) is a poorly differentiated neuroendocrine (NE) neoplasm accounting less than $1 \%$ of all bladder tumors, $1 / 3$ are admixed with conventional urothelial carcinoma and hematuria is the most common clinical presentation. The unknown etiology and natural history of SCC represent a challenge for its diagnosis and treatment. $\operatorname{Aim}(\mathbf{s})$ : Study the clinical and pathologic characteristics of SCC of the urinary bladder. Materials and Methods: Clinicopathological data from 12 cases of pure or mixed SCC diagnosed in our center with immunohistochemical stains (IHC) to confirm NE differentiation. Results: Histological samples were transurothelial resection biopsies (TUR) in 10 cases and radical cystectomy in 2 cases. Two of the 10 cases with TUR underwent surgical resection. Six cases were diagnosed as pure SCC and 6 cases as mixed SCC with other subtypes. Final patient staging was 1 pT4, 2 pT3, 7 pT2, 1 pT1 and 1 not specified. Adjuvant treatment was chemotherapy (CT) alone in 2 cases, radiotherapy (RT) alone in 2 cases, CT and RT in 2 cases and BCG in 1 case. Nine patients died in an average of 4 months. All cases were positive for synaptophisin, other NE staining (chromogranin, NSE and CD56) showed heterogeneous results. Conclusion: Bladder SCC is a highly malignant disease, associated with poor prognosis. The morphology as well as the use of IHC is important to identify this neoplasm. The identification of co-existing urothelial carcinoma or in situ component supports the bladder primary. Keywords: small cell, bladder.
P6

Characterization and Prognosis of Patients with Metastatic Lung Carcinoid Tumors

Walter T. ${ }^{\mathrm{a}}$, Planchard D. ${ }^{\mathrm{b}}$, Bouledrak K. ${ }^{\mathrm{a}}$, Guigay J. ${ }^{\mathrm{c}}$, Dussol A.S. a, Scoazec J.Y. ${ }^{\mathrm{b}}$, Hervieu V. ${ }^{\mathrm{a}}$, Ducreux M. ${ }^{\mathrm{b}}$, Lombard-Bohas C. a, Baudin E.

aHospices Civils de Lyon, Hôpital Edouard Herriot, Lyon, France; ${ }^{b}$ Gustave Roussy, Villejuif, France; ' Gustave

Roussy, Lyon, France

Introduction: Data on metastatic lung carcinoid tumors are limited. Aim(s): The study aimed to describe characterization and prognostic factors of metastatic carcinoid lung tumors (CLT). Materials and Methods: All consecutive patients with a metastatic (synchronous or metachronous) CLT referred to two tertiary centers between January 1999 and January 2013 were included in this retrospective study. Results: 117 patients (59\% male, aged of 58 (20-84) years) were included. Metastases were metachronous in $55 \%$ of patients, occurring 51 (2-224) months from initial diagnosis. At metastatic diagnosis, $92 \%$ were PS $0-1,46 \%$ had functioning tumors (48 carcinoid syndrome, 3 Cushing, 1 PTHrp, 1 GHRH). The median number of metastatic sites was 2 (1-6), mainly in the liver (87\%) and bones $(52 \%)$. 18F-FDG TEP scan and Octreoscan ${ }^{\circledR}$ were positive in 24/27 (89\%) and 56/70 (80\%). Pathological data showed: $21 \%$ of typical carcinoid, $46 \%$ of atypical carcinoid and $33 \%$ of well differentiated NET on metastatic biopsy. Tumors grades were $46 \%, 36 \%$ and $18 \%$ for grade $\mathrm{G} 1, \mathrm{G} 2$, and G3, respectively. The median overall survival from metastatic diagnosis was 6.6 (5.3-7.9) years. After univariate analysis, high performance status, brain metastasis, bone metastasis, and atypical carcinoids were associated with worse OS. $24 \%$ underwent surgery of their metastases, $34 \%$ a transarterial liver embolization, and $75 \%$ received somatostatin analogs. Conclusion: Metastatic CLT have some similarities with gastro-intestinal carcinoids tumors but with worse outcome. Keywords: lung, metastatic, prognosis. 


\section{Clinical Cases/Reports}

\section{Q1 \\ Use of Everolimus in a Patient with Metastatic Bronchial Carcinoid Receiving Hemodialysis}

\author{
Brizzi M.P. ${ }^{a}$ Sonetto C. ${ }^{\text {a }}$ Bitossi R. ${ }^{\text {a }}$ Baratelli C. ${ }^{\text {, }}$ \\ Volante M. ${ }^{\text {b }}$ Tampellini M. ${ }^{\text {a }}$, Scagliotti G. V. ${ }^{\text {a }}$ \\ a Medical Oncology, Department of Oncology, University \\ of Turin at San Luigi Hospital, Orbassano, Turin, Italy; \\ bPhatology, Department of Oncology, University of Turin at \\ San Luigi Hospital, Orbassano, Turin, Italy
}

Introduction: Renal impairment may alter the normal pharmacokinetic and pharmacodynamic behavior of chemotherapy drugs, thus increasing the risk of side effects. Everolimus was reported to have anti-tumor activity in bronchial neuroendocrine tumors (NET). Safety and toxicity profile of everolimus has been provided in hemodialyzed patients with renal cell carcinoma. Only a report describes the effect of everolimus in a patient with metastatic ileac carcinoid in hemodialysis. Aim(s): To test the feasibility of everolimus in a patient with metastatic atypical bronchial carcinoid receiving hemodialysis. Materials and Methods: A 47 year-old man suffering from a metastatic atypical bronchial carcinoid, in hemodialysis, had radiologic and clinical progressive disease after treatment with somatostatin analogues. Patient received oral everolimus. Results: The drug was administered at $10 \mathrm{mg} /$ day only for 10 days, due to the occurrence of grade II mucosites. The treatment was carried on the dose of $5 \mathrm{mg}$ daily. Everolimus was discontinued after six months of treatment for disease progression. Other CTCAE (Common Terminology Criteria for Adverse Events) grade I adverse events included hiporexia and cholesterol level increase. No grade III-IV toxicities occurred. Conclusion: Oral everolimus is feasible regimen in the management of patient with metastatic well differentiated bronchial neuroendocrine, receiving hemodialysis. Renal impairment may require a drug dose adjustment to achieve an excellent tolerance. Keywords: bronchial carcinoid, everolimus, hemodialysis.
Q2

\section{Mixed Adenoneuroendocrine Carcinomas (MANECs) of the Gastrointestinal Tract. ARGENTUM Group}

\author{
Cabanne A. a, Bestani C. ${ }^{\text {a }}$ Pesce V. ${ }^{\text {a }}$ O'Connor J.M. ${ }^{\text {b }}$ \\ Kujaruk M. ${ }^{a}$, Domenichini E. ${ }^{b}$, Belli S. ${ }^{c}$, Rosales C. , \\ Roca E. \\ ${ }^{a}$ Gastroenterology Hospital, B. Udaondo, Buenos Aires, \\ Argentina; 'bleming Institute, Buenos Aires, Argentina; \\ 'Durand Hospital, Buenos Aires, Argentina; ${ }^{\mathrm{d}}$ Marie Curie \\ Hospital, Buenos Aires, Argentina
}

Introduction: Mixed adenoneuroendocrine carcinomas of the gastrointestinal tract are rare neoplasms. In the 2000 WHO classification of endocrine tumors, such neoplasms were defined as mixed exocrine-endocrine tumors when each component represents at least $30 \%$ of the lesion. In the most recent WHO classification, such neoplasms are called 'mixed adenoneuroendocrine carcinomas' (MANECs). They are a challenge for the diagnostic and treatment. $\operatorname{Aim}(\mathbf{s})$ : To evaluate the clinical and histopathological characteristics of MANECs. Materials and Methods: We review all cases of MANECs of database Argentum group. The MANEC diagnosis was confirmed by conventional histopathology on surgical specimen. Ki-67 analysis was done on paraffin-embedded sections using immunohistochemistry. Grading was according to ENETS recommendations. Results: We identified 6 MANECs (primary tumor: 3 colon, 2 rectum and 1 esophagus). M:F (3:3), age 19-69 years (mean:43.5). Pts $66.7 \%$ had localized or regional disease, and $33.3 \%$ metastatic disease. Histologically, they were adenocarcinoma or squamous carcinoma with a NET component (G:1/6; G2:3/6; G3:2/6). 3 were resected according to oncological criteria for adenocarcinoma; the others performed chemotherapy \pm radiotherapy. Conclusion: MANECs of the of the gastrointestinal tract are rare neoplasms and the optimal strategy of management of MANECs is largely unknown. The prognosis is determined by the most aggressive tumor component. The treatment recommendations should be according to the adenocarcinoma or NECs. Keywords: manec, nets.

\section{Q3}

\section{Gastric Neuroendocrine Neoplasms and Long-Term Proton Pump Inhibitors: Need for a Revision of Classifications?}

\author{
Cavalcoli F. ${ }^{\mathrm{a}}$, Zilli A. ${ }^{\mathrm{a}}$, Conte D. ${ }^{\mathrm{b}}$, Ciafardini C. ${ }^{\mathrm{a}}$, \\ Massironi a $^{\text {a }}$ \\ 'Fondazione IRCCS Ca' Grande-Ospedale Maggiore \\ Policlinico, Gastroenterology and Endoscopy Unit, Milan, \\ Italy; 'bepartment of Pathophysiology and Transplant, \\ Università degli Studi di Milano, Milan, Italy
}

Introduction: Gastric neuroendocrine neoplasm (gNENs) include three types: type 1 and 2 related to hypergastrinemia due to chronic atrophic gastritis (CAG) or Zollinger-Ellison syndrome (ZES) respectively, and type 3 normogastrinemic and more aggres- 
sive. Few cases of gNENs in patients taking proton pump inhibitors (PPI) have been reported. Aim(s): To describe two cases of gNENs in patients on long-term PPI and not classifiable according to current criteria. Materials and Methods: From January 2005 to July 2014, 30 gNENs were referred to our Unit. Out of these, $24(80 \%)$, one $(3.3 \%)$ and three $(10 \%)$ had type 1,2 and 3 respectively. Two cases $(6.7 \%)$ were undetermined according to current classifications. Results: Both patients had long-term PPI intake due to gastroesophageal reflux disease. Patient 1: a 58-year-old man with a $6 \mathrm{~mm}$, well differentiated gNEN (Ki-67 $=4 \%$ ), limited to muscolaris mucosae. Patient 2: a 78 -year-old man, with a $4 \mathrm{~mm}$, gNEN (Ki$67<1 \%)$. This patient presented marked hypergastrinemia $(1250 \mathrm{pg} /$ $\mathrm{ml}$; r.v. <108), which normalized after PPI discontinuation. In both cases CAG, H.pylori infection and ZES were excluded. At histology ECL-hyperplasia was observed in both patients, in the first case cystic polyps were reported. PPI were discontinued and thr patients had endoscopic polypectomy, follow-up was negative for recurrence or CAG. Conclusion: Our data indicate the epidemiological relevance of gNENs associated with PPI. These neoplasms escape from current classification, suggesting the need for its revision. Keywords: ppi, gnen, classification, cag.

\section{Q4 \\ Second Primary Malignancies in Patients with Neuroendocrine Tumors}

Clift A., Drymousis P., Al-Nahhas A., Wasan H., Martin J., Frilling $A$.

Imperial College London, London, UK

Introduction: Numerous studies have demonstrated an elevated incidence of second primary malignancies (SPM) in patients with neuroendocrine tumours (NET). Aim(s): To review our institutional experience of SPM in NET patients. Materials and Methods: Individual patient notes were obtained: data regarding gender, age, date and type of diagnoses were extracted. Patients with any tumour pre-disposition syndrome were excluded. SPM were classed as previous, synchronous, or metachronous relative to NET diagnosis. A person-year methodology was used to compare the observed frequencies of SPM against expected values generated using age- and sexspecific incidence tables, with standardised incidence ratios (SIRs) and 95\% confidence intervals (CI) calculated. Results: 169 patients were included, with 29 SPM observed in 26 (15.38\%) patients. Colorectal $(n=6)$, renal and breast cancers (both $n=5)$ were the commonest SPM. There were 19 previous SPM in 16 patients $(9.47 \%)$, synchronous SPM in $8(4.73 \%)$ and metachronous SPM in $2(1.18 \%)$. SIRs $(95 \% \mathrm{CI})$ for previous, synchronous, and metachronous SPM were: 0.4 (0.241 to 0.624$), 2.732$ (1.177 to 5.382), and 0.36 (0.044 to $1.051)$, respectively. For synchronous colorectal SPM: SIR $=13.079$ (95\% CI: 4.238 to 30.474 ). Conclusion: We demonstrate a statistically significant increased risk of synchronous SPM, particularly colorectal carcinomas. We recommend that routine colonoscopy be incorporated into the diagnostic work-up of NET patients in anticipation of a colorectal SPM. Keywords: neuroendocrine, neoplasm, second, cancers.
Q5

\section{A Novel Approach for a Metastatic Gut-Derived Neuroendocrine Tumour: Simultaenous Intestinal and Vascularised Sentinel Forearm Flap Transplantation}

\author{
Clift A. ${ }^{\mathrm{a}}$, Giele H. ${ }^{\mathrm{b}}$, Vrakas G. ${ }^{\mathrm{b}}$, Reddy S. ${ }^{\mathrm{b}}$, Macedo R. ${ }^{\mathrm{b}}$, \\ Al-Nahhas A. ', Wasan H. ${ }^{\text {a }}$ Gondolesi G. ', Vianna R. ${ }^{\mathrm{d}}$, \\ Friend . $^{\mathrm{b}}$, Vaidya A. ${ }^{\mathrm{b}}$, Frilling $A{ }^{\mathrm{a}}$ \\ amperial College London, London, UK; ${ }^{\text {b Oxford }}$ \\ University NHS Trust, Oxford, UK; ' Instituto de Trasplante \\ Multiorganico, Buenos Aires, Argentina; ${ }^{\mathrm{d} M i a m i}$ \\ Transplant Institute, Miami, Fla., USA
}

Introduction: Non-resectable tumours involving the mesenteric root are an indication for intestinal and multivisceral transplantation (MVT). Vascularised sentinel forearm flaps (VSFF) offer possibilities for monitoring graft rejection and tailoring immunosuppression (IS) regimens. Aim(s): We present the first documented case of using MVT for a metastatic neuroendocrine tumour (NET) whilst implementing a VSFF. Materials and Methods: A 44 year old male was diagnosed with a well-differentiated Grade $1($ Ki67 $<1 \%)$ NET. Serum chromogranin A was $395 \mathrm{pmol} / \mathrm{L}$ (reference range 0-60), chromogranin B was $349 \mathrm{pmol} / \mathrm{L}$ (reference range 0-150), and 5-hydroxyindoleacetic acid in $24 \mathrm{hr}$ urine was $643 \mu \mathrm{mol} / \mathrm{L}$ (reference range 0-40). 68Ga-DOTATATE PET/CT showed significant uptake in mesenteric tumour bulk and in an aorto-caval lymph node. At laparotomy, stage IV tumour bulk encasing the mesenteric root, numerous enlarged lymph nodes near the small bowel loops, and 7 sub-centimetre tumours scattered over the ileum were observed. Results: After 4 cycles of 177Lu-PRRT, he underwent simultaneous VSFF and a modified MVT involving exenteration of the stomach, pancreas, spleen, small bowel and colon (left hemicolon spared) with extirpation of an aorto-caval lymph node. Disease was staged as pT3 N1 M0 L1 V0 R0. Conclusion: 17 months post-transplant, he is asymptomatic on IS and back to full physical activity. One mild rejection reaction in the VSFF was treated. There has been no rejection in the bowel, and no disease recurrence. Keywords: neuroendocrine, tumor, transplantation. 
Q6

\section{Regression of Paraneoplastic Optic Neuropathy Associated with Glucagonoma after Surgical Resection}

\author{
Defour L. a, De Mestier L. a, Diebold M.D. ${ }^{\text {b }, ~ A r n d t ~ C . ~}{ }^{\text {, }}$, \\ Kianmanesh R. ${ }^{\mathrm{d}}$, Cadiot G. ${ }^{\mathrm{a}}$ \\ ${ }^{a}$ Department of Hepato-Gastroenterology and Digestive \\ Oncology Hôpital Robert-Debré, Reims, France; \\ bDepartment of Pathology Hôpital Robert-Debré, Reims, \\ France; 'Department of Ophtalmology, Reims, France; \\ ${ }^{\mathrm{d} D e p a r t m e n t}$ of Digestive and Endocrine Surgery Hôpital \\ Robert-Debré, Reims, France
}

Introduction: Glucagonomas-associated ocular manifestations have been exceptionally reported. Aim(s): To report the case of a patient with a glucagonoma associated with paraneoplastic optic neuropathy $(\mathrm{PON})$ and to review literature. Materials and Methods: A 65-year-old man presented with bilateral visual loss and central scotoma. Later, the occurrence of diarrhoea, necrolytic migratory erythema evocated a glucagonoma syndrome, which was advocated by elevated chromogranin A and glucagon levels. CT scan showed a pancreatic tumor, which was confirmed to be a well-differentiated neuroendocrine tumor at pathological examination of biopsies. Surgical resection was performed and led to the dramatic improvement of bilateral visual acuity, and complete resolution of scotomas. Hence, the retained diagnosis was a pancreatic glucagonoma associated with a PON. Results: The association of PON with pancreatic neuroendocrine tumors in general, and glucagonomas in particular, has been specifically described 6 and 4 times in the literature, respectively. In larger series, glucagonomas-associated ophtalmological manifestations were reported in up to $14 \%$ of cases. Although never described in the setting of glucagonomas, the anti-CRMP5 IgG can be found in the serum of patients affected with paraneoplastic neurological syndromes in general, and those with PON in particular. Conclusion: Practicians should be aware of the possible association of glucagonomas with PON and regression of PON after tumour resection. Keywords: paraneoplastic syndrome, glucagonoma, optic neuropathy.

\section{Q7 \\ Clinicopathologic Characteristics of Gastric Amphicrine Carcinoma at a Single Institution}

Huang D., Sheng W.

Fudan University Shanghai Cancer Center, Shanghai, China

Introduction: Amphicrine carcinoma that shows evidence of duel neuroendocrine and epithelial differentiation occurs in the same cell is rare. Aim(s): We report 4 cases of amphicrine carcinoma in the stomach with their clinicopathologic findings. Materials and Methods: Clinical and pathologic feature were collected and analyzed. Mucin-stains and immunohistochemistry, Cytokeratin, Synaptophysin (SYN), Chromogranin A (CGA) and Somatostatin Receptor 2 (SSTR2), were performed. Results: All four patients with amphicrine carcinoma were male. The median age at presentation was 59 years (range, 56-61 years). The size of the tumors ranged from $2 \mathrm{~cm}$ to $5.5 \mathrm{~cm}$ (median, $3.3 \mathrm{~cm}$ ). Histopathological examination showed the tumours were composed of small, rounded nests of signet ring like cells resembling normal intestinal goblet cells, except for the nuclear compression. The cells displayed mild-to-moderate atypia with low mitotic activity. Mucin staining is intensely positive within goblet cells and extracellular mucin pools. CGA, SYN and SSTR2 immunoreactivity showed diffuse strong staining throughout the sections in all cases. Late stage was present in all patients, and the overall survival ranged from 16 months to 38 months. Conclusion: Rigorous interpretation of dual immunohistochemical expression of neoplastic cells is useful in distinguishing the amphicrine carcinoma from others with similar morphological characteristics. However, in this entity, the behavior as well as the treatment currently remains in dispute. Keywords: amphicrine carcinoma, stomach, pathology.

Q8 A Novel MEN1 Gene Variant in a Sporadic
Case of Multiple Endocrine Neoplasia Type 1

lacovazzo D. ${ }^{a}$, Piacentini S. ${ }^{a}$, Lugli Fa , Bianchi A. ${ }^{a}$, Gabrovska P. , Persiani R. ${ }^{\mathrm{c}}$, Rindi G. ${ }^{\mathrm{d}}$, Ellard S. ${ }^{\mathrm{e}}$, Korbonits M. ${ }^{b}$, De Marinis L. ${ }^{a}$

aEndocrinology-Università Cattolica del Sacro Cuore, Rome, Italy; ${ }^{b}$ Endocrinology-Barts and The London School of Medicine, London, UK; ' General SurgeryUniversità Cattolica del Sacro Cuore, Rome, Italy; dPathology-Università Cattolica del Sacro Cuore, Rome, Italy; 'Molecular Genetics-Royal Devon and Exeter NHS Foundation Trust, Exeter, UK

Introduction: Multiple endocrine neoplasia type 1 (MEN1) is an autosomal dominant disorder characterised by the occurrence of hyperparathyroidism, pituitary adenomas and duodeno-pancreatic neuroendocrine tumours (NETs). Germline mutations of the MEN1 gene are identified in up to $87 \%$ of MEN1 families and $82 \%$ of isolated MEN1 cases. Aim(s): To report a novel variant of the MEN1 gene. Materials and Methods: We describe a typical sporadic MEN1 patient. Mutational analysis of the MEN1 gene (exons 2-10) has been performed by Sanger sequencing. Results: A 61-year old male patient presented with melena due to a multifocal neuroendocrine tumour of the stomach, later found to be a type 2 gastric carcinoid associated with a duodenal gastrinoma. A non-functioning subcentimetric pancreatic NET was also found. Biochemistry showed hypercalcaemia due to primary hyperparathyroidism. Pituitary MRI identified a non-functioning microadenoma. The patient underwent total gastrectomy and duodeno-cephalo-pancreatectomy. Sub-total parathyroidectomy was later performed. Mutational analysis of the MEN1 gene revealed a novel heterozygous in-frame deletion of 15 base pairs in exon 2 (c.286_300del, p.Q96_A100del). This variant causes the loss of 5 highly-conserved aminoacids in the Smad3 binding domain of menin and is predicted in silico to be deleterious. Conclusion: The clinical picture and in silico prediction strongly suggest the pathogenic effect of this novel MEN1 gene variant. Keywords: men1, mutation, gastric carcinoid. 
Q9

Fifty-Three Year-Old-Male with a Symptomatic lleocecal Carcinoid: An Individual Follow-Up of 14 Yrs with 5 Different Anti-Proliferative Therapies

Kump P., Suppan C., Spindelböck W., Krejs G., Lipp R.

Medical University Graz, Graz, Austria

Introduction: Only limited reports exist about sequence of different anti-tumor therapies in patients with neuroendocrine tumors (NETs) after primary surgery. $\mathbf{A i m}(\mathbf{s})$ : The present case summarizes a 13 years history of various therapies in a patient with NET. Materials and Methods: In 2001 a 53-year-old patient presented with carcinoid syndrome due to an ileocecal NET (pT3 N2 G1). 4 years after hemicolectomy. bilobular liver metastases were resected due to stage IV disease. Results: Despite palliative surgery liver metastasis progressed and Sandostatin LAR $20 \mathrm{mg}$ was initiated in 2005. The disease was stable for 39 month. In 2008, therapy was extended with 3 cycles of $90 \mathrm{Y}-$ and $177 \mathrm{Lu}$-octreotate after progression of liver mets and newly presented lymph nodes, and stabilized disease for another 33 month. In 2011, progression of liver mets, lymph nodes, and soft tissue involvement led to an inrease of Sandostatin LAR to $30 \mathrm{mg}$, but 3 months later new lesions in bone and peritoneum were shown. Everolimus (Affinitor $10 \mathrm{mg}$ ) was now added to Sandostatin and stabilized tumor growth for $37 \mathrm{mo}$. When the tumor progressed again in 2013, targeted therapy was switched to sunitinib (Sutent $50 \mathrm{mg}$ ), however, the disease progressed further, Sandostatin LAR was maximized to $40 \mathrm{mg}$, and targeted therapy changed to chemotherapy with capecitabine and temozolomide (CAPTEM) 7/2014. Conclusion: This case shows the promising sequel of 5 different therapies in intestinal G1 NET. Keywords: targeted therapy, sequence therapy, neuroendocrine tumor, gep-net.

\section{Q10}

Pancreatic Neuroendocrine Tumor and lleal Carcinoid in Acromegaly. Pluriglandular Association in Non-MEN 1 Patient: A Case Report

Lico V. ${ }^{\text {a, }}$ Milanetto A.C. ${ }^{\text {a }}$ Moletta L. ${ }^{\text {, }}$ Scaroni C. ${ }^{\text {, }}$, Alaggio R. ${ }^{\mathrm{c}}$, Pasquali $C$. $^{\text {a }}$

apancreatic and Digestive Endocrine Surgical Unit, Padua, Italy; ${ }^{b}$ Endocrinology, Padua, Italy; 'Pathology, Padua, Italy

Introduction: Pluriglandolar endocrine disease in patients without familial inherited disease (non-MEN 1) is very rare. Primary Pancreatic Neuroendocrine Tumors (pNET) and ileal carcinoid tumors have different embryologic origin (foregut and midgut respectively). Aim(s): To report the clinical and pathological features of a patient with syncronous GH secreting pituitary adenoma, primary pNET and ileal (midgut) carcinoid. Materials and Methods: A 66 years old man with acromegaly due to a GH-secreting pituitary adenoma, was referred to our center because of the incidental finding at CT-scan of a $8.7 \mathrm{~cm}$ round cystic lesion at the pancreatic tail. It was detected also a $3 \mathrm{~cm}$ solid hypervascular lesion in the mesenteric context and a hypervascular lesion in the small gut suggesting a midgut carcinoid with lymph-node metastasis. 68Ga-DOTA-NOC PET/ CT showed focal uptake in all abdominal lesions confirming their NE nature. High serum 5HT levels were found while urinary 5-HIAA was normal. Results: The patient underwent distal splenopancreatectomy, ileal resection and excision of the mesenteric lesion. The histology and immunohistochemical examinations confirmed that the cell pattern of the pancreatic NET $(5$-HT negative, Ki-67 <1\%) was different from the ileal carcinoid with lymph node metastases. The patient is well 26 months after surgery without recurrence. Conclusion: Syncronous neuroendocrine tumor of pancreas (pNET), midgut carcinoid and pituitary adenoma is a very rare association and in this case was MEN 1 unrelated. Keywords: acromegaly, carcinoid, pnet.

\section{1 \\ 68 Yr Female with a Growing Inoperable Myocardial Metastase}

\author{
Linder Ekberg $K$. \\ Karolinska University Hospital, Stockholm, Sverige
}

Introduction: Two recent studies shows that cardiac metastases occur rather frequently, $13 \%$ in a population of serotoninproducing NETs (Noordzij 2014) and 4\% in our own patient kohort of ileal NETs (Calissendorff 2014). Aim(s): Present an unusual case of NET metastase. Materials and Methods: This woman presented 2009 at the age of 63 with episodes of vertigo and headaches. A CT scan of the head was normal but transthoracic echocardiography performed in search of an embolic source showed a $5 \mathrm{~cm}$ structure in the vicinity of the right ventricle. MRI demonstrated that that the structure was lodged within the myocardium and with the same signal intensity pattern as myocardium. Results: Open heart biopsy revealed a NET with a Ki-67-index of $<1 \%$. The patient's tumor markers were elevated, she had frequent flushes but no diarrhea. SRS and Gallium68DOTATOC-PET/CT showed the cardiac metastase, an ileal primary tumor and a few small skeletal metastases but no liver engagement. The cardiac metastase was judged inoperable. The primary tumor was resected dec 2010 together with mesenteric metastasis and showed a $\mathrm{Ki}-67$ of $<0.1 \%$. Conclusion: She was stable with somatostatin analogues and interferon until spring 2014 when she hade neutropenia and interferon treatment was closed. During spring 2014 she develops rightsided heart failure and echocardiography shows no obstruction but a reduced right ventricular function due to tumor growth in the wall. She is now referred for PRRT. We search experience of similar case. Keywords: myocardiac heart metastase, net, ileal net. 
Q12

\section{Neuroendocrine Pancreatic Tumor Associated with a Cerebral Neurofibroma. A Case Report}

\author{
Moletta L. ${ }^{a}$, Milanetto A.C. ${ }^{\text {a }}$ Lico V. ${ }^{\text {, }}$ Farinati F. ${ }^{\text {, }}$ \\ Alaggio R. ${ }^{c}$, Pedrazzoli S. ${ }^{a}$, Pasquali C. ${ }^{a}$ \\ aPancreatic and Digestive Endocrine Surgical Unit, Padua, \\ Italy; 'bepartment of Gastroenterology, Padua, Italy; \\ cDepartment of Pathology, Padua, Italy
}

Introduction: Neuroendocrine pancreatic tumors (pNET) are rare in young patients. They usually occur in the setting of genetic syndromes. Aim(s): To report the clinical and pathological features of a young patient with pNET associated with cerebral neurofibroma. Materials and Methods: A 25-years old man with a long history of chronic abdominal pain and diarrhoea was observed in May 2006. A cerebral (right frontal) $1 \mathrm{~cm}$ lesion, stable in site, was known since 1996. A duodenal biopsy of a $2.5 \mathrm{~cm}$ lesion close to the papilla showed NE tissue. Octreoscan scintigraphy was positive in pancreatic head/duodenum. EUS confirmed a $3 \mathrm{~cm}$ pancreatic head mass infiltrating the duodenum with main duct dilation and stenosis of common bile duct. Exocrine pancreatic insufficiency with low fecal elastase $(98 \mathrm{ug} / \mathrm{g})$ was present. At laparotomy was showen a jejunal invagination due to Meckel's diverticulum and a pancreatic head lesion. A pancreaticoduodenectomy and Meckel resection were performed. Results: Histology showed a G2 pNET with one lymph-node metastasis $(\mathrm{Ki}-67=2.2 \%$, MI $=7 / 10 \mathrm{HPF})$ with positive Somatostatin immunostaining. Three years later the cerebral lesion was removed as it grew up to $5 \mathrm{~cm}$ and was found to be a neurofibroma. A neurofibromatosis type 1 (NF-1) was suspected but the patient refused genetic tests. He is still alive, free from disease, 91 months after surgery. Conclusion: pNET arising in NF-1 patients is a rare occurrence and may behave malignant. NF-1 should be taken in account in young patients. Keywords: pnet, cerebral neurofibroma, nf-1.

\section{Q13 \\ Case Report of Recurrent VIPoma \\ Mulcahy V., McStay M., Sizer B. \\ Colchester General Hospital, Colchester, UK}

Introduction: $51 \mathrm{yr}$ presented with 7 month diarrhoea\&2 stone weight loss. All tests negative, only abnormality hypokalaemia. FGH profile: VIP raised $>400$, peptide histidine methionine $>1000$, GAWK of 250. Diagnosis of VIPoma. CT: $5 \mathrm{~cm}$ mass tail \& body of pancreas, no hepatic or adrenal lesions. Octreotide treatment started. Bowel frequency improved, hypokalaemia corrected. Octreotide scan showed uptake only in pancreas. Patient readmitted 5 months later hypokalaemic myopathy, K 1.7, surgical distal pancreatectomy \& splenectomy completed. Histology: NET within pancreas, moderate cellular differentiation, variable mitotic rate. Aim(s): Tumour enclosed in capsule, showed capsular invasion. Patient discharged, followed-up $9 \mathrm{yr}$, no signs of recurrence. Materials and Methods: $11 \mathrm{yr}$ on patient referred on cancer pathway with diarrhoea, no elec- trolyte changes. Colonoscopy: normal. CT: tumour recurrence in pancreatic bed. CgA120, CgB89, VIP130, other FGH normal. Ocreotide scan: uptake left upper quadrant \& epigastrium. USS biopsy: NET minimal cellular differentiation, no evidence mitotic activity, positive chromogranin \& synaptophysin, low Ki67 <5\%. Results: Surgical resection of reoccurrence completed, no signs of peritoneal spread. Histology: tumour in thin fibrous capsule, invasion into the capsule. Cells positive for NE markers. Negative for $\mathrm{Cg}$. Proliferation marker patchy distribution: $5 \%-15 \%$. Patient under close surveillance. Conclusion: Distinction of differentiation of NETs is one of most important pathologic assessment in planning treatment. Keywords: vipoma.

Q14

\section{Simultaneous Large Cell Neuroendocrine Carcinoma and Adenocarcinoma of the Stomach: A Case Report and Literature Review}

Nakazuru S. ", Matsumura Y. , Harada Y.b , Iwasaki R. ${ }^{\text {a }}$ Iwasaki T. ${ }^{a}$, Nishio K. ${ }^{a}$, Hasegawa H. ${ }^{a}$, Sakakibara Y.' Yamada T. ${ }^{a}$, Nishikawa K. ${ }^{b}$, Ishida H. ${ }^{a}$, Mori K. ${ }^{c}$, Mita E. ${ }^{a}$

aDepartment of Gastroenterology and Hepatology, Osaka National Hospital, Osaka, Japan; 'Department of Surgery, Osaka National Hospital, Osaka, Japan; 'Department of

Pathology, Osaka National Hospital, Osaka, Japan

Introduction: Large cell neuroendocrine carcinoma (LCNEC) of the stomach is rare. Gastric NEC occasionally has another component, such as adenocarcinoma, in the same tumor. However, the concurrent occurrence of LCNEC and adenocarcinoma at different sites in the stomach is extremely rare. $\mathbf{A i m}(\mathbf{s})$ : We present a case of simultaneous LCNEC and tubular adenocarcinoma of the stomach, and review the literature. Materials and Methods: An 80-yearold man was previously admitted to a hospital for tarry stools. EGD showed an ulcerative lesion on the greater curvature of the lower body of the stomach. The pathological diagnosis based on the biopsy was moderately differentiated adenocarcinoma. He was referred to our hospital for gastrectomy. Results: Distal gastrectomy with lymph node dissection was performed. The resected stomach had a type 2 tumor and a type 0 -IIa tumor. Histological examination of the resected specimen confirmed the diagnosis of LCNEC and well differentiated tubular adenocarcinoma at different sites in the stomach. The Ki-67 labeling index for LCNEC was 39\%. Ten cycles of irinotecan plus cisplatin were administered as adjuvant chemotherapy. After 30 months, CT showed 2 liver metastases. Partial hepatectomy was performed. Conclusion: To our knowledge, only one case of simultaneous LCNEC and adenocarcinoma of the stomach has been published to date. This report presents a rare surgical case of synchronous LCNEC and early adenocarcinoma of the stomach. Keywords: stomach, large cell neuroendocrine carcinoma, tubular adenocarcinoma, simultaneous. 


\section{5}

\section{Two Cases of Ectopic ACTH-Secreting Neoplasm of Occult Origin Becoming Overt after Bilateral Adrenalectomy}

\author{
Piacentini S. a, lacovazzo D. a, Lugli Fa, Giampietro A. a, \\ Bianchi A. a, Tartaglione L. ${ }^{a}$, Mormando M. ${ }^{\text {, }}$ Chiloiro S. ${ }^{\text {, }}$ \\ Raffaelli M. ${ }^{\mathrm{b}}$, De Marinis L. ${ }^{\mathrm{a}}$ \\ ${ }^{a}$ Endocrinology-Università Cattolica del Sacro Cuore,

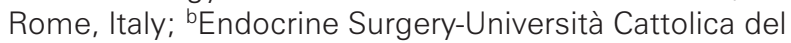 \\ Sacro Cuore, Rome, Italy
}

Introduction: Cushing's syndrome (CS) is an endocrine disorder associated with serious consequences if not adequately treated. Nearly $80 \%$ of the cases of endogenous hypercortisolism are adrenocorticotropic hormone (ACTH) dependent, the ACTH source being pituitary in about $70-80 \%$ and ectopic in $10-15 \%$. The ACTH source may remain occult in few cases for many years in spite of extensive investigations. Aim(s)/Materials and Methods: we describe a retrospective series of 13 patients affected by ectopic ACTH dependent CS. The origin of ectopic ACTH secretion was: bronchial carcinoid for 8 patients, 1 atypical thymic carcinoid and in 4 cases the origin remained occult. Results: in $9 / 13$ cases the ectopic origin was found ( 8 bronchial carcinoid and 1 thymic carcinoid), while in 4 patients the source of ACTH secretion remained unknown. Regarding the latter 4 patients with unknown origin of disease, all of them underwent bilateral adrenalectomy after several years of medical treatment with poor control of the clinical syndrome. In 2 patients, about one year after the adrenalectomy, the ACTH-secreting neoplasm became overt (1 pancreatic neuroendocrine tumor and 1 breast cancer), and in both cases the neoplasms positively stained for ACTH. The remaining 2 patients are still in follow up and the primary site of ACTH secretion is still hidden. Conclusion: these patients need to be closely followed up, as the source of ACTH secretion might rapidly be come overt after the adrenalectomy. Keywords: cushing's syndrome, ectopic acth, bilateral adrenalectomy.

\section{Q16}

\section{Hypoglycaemia in a Patient with Glucagonoma-Coincidence of a Glucagonoma and Insulinoma in a Patient with Multiple Endocrine Neoplasia Type I}

Ploner F. , Plank J. ${ }^{\mathrm{b}}$, Humer-Fuchs U. ${ }^{c}$, Lax S. ${ }^{c}$, Noggler P. Rosanelli G. ${ }^{\text {, Stepan V. }}$

aDepartment of Internal Medicine/Oncology, Medical University Graz, Graz, Austria; 'b Department of Internal Medicine/Endocrinology, Medical University Graz, Graz, Austria; 'Institut of Pathology, LKH West, Graz, Austria; dDepartment of Surgery, Elisabethinen Hospital Graz, Graz, Austria; eDepartment of Internal Medicine, Elisabethinen Hospital Graz, Graz, Austria

Introduction: We report the case of a 54 year old female with a known MEN1 syndrome with primary Hyperparathyroidism, a Prolactinoma and a pancreatic tumor. The patient complains about episodes of fatigue and sweating, both symptoms improve immediately after a meal. Aim(s): Lab tests show a fasting glucose of $45 \mathrm{mg} /$ $\mathrm{dl}$, (without hypoglycaemic symptoms). A MRI of the pancreas shows a tumor with typical radiologic characteristics of a pancreatic neuroendocrine Tumor (PNET) with an increasing diameter from 22 to 27 $\mathrm{mm}$ (compared to one year ago). Further lab tests show an elevated glucagon level with $942 \mathrm{pg} / \mathrm{ml}(-250 \mathrm{pg} / \mathrm{ml})$. Insulin and C-peptide values were in the lower normal range. Materials and Methods: Due to the clinical symptoms and the increase in tumor size a left sided pancreatectomy and splenectomy was performed. Results: Histological and immunohistochemical examination shows a 2.9 cm PNET (pT-2, G-1, pN-0) with a highly positive reaction against Insulin-, and no reaction with Glucagon-antibodies. Next to this tumor another PNET (4 mm) was detected, which was highly positive against Glucagon-, but showed no reaction to Insulin-antibodies. After surgery the patient had no more hypoglycaemic episodes, and showed normal fasting glucose levels. Conclusion: There are only very few reports about the coexistence of a glucagonoma and an insulinoma. Our patient showed the suprising combination of a clinical apparent Insulinoma with hypoglycaemic episodes and low normal Insulin values, but significantly elevated glucagon levels. Keywords: men1, glucagonoma.

\section{Q17 \\ Staged Percutaneous Treatment of Severe Carcinoid Heart Disease by Transfemoral Pulmonary Valve Replacement and Novel Vena Cava Valve Implantation}

Pschowski R. ${ }^{\text {, }}$ Laule M. ${ }^{\mathrm{b}}$, Baur A. ${ }^{\mathrm{c}}$, Pape U.F. ${ }^{\mathrm{a}}$, Pavel M. ${ }^{\mathrm{d}}$ Stang/ V. ${ }^{\mathrm{b}}$, Wiedenmann B. ${ }^{\mathrm{a}}$, Stang/ K. ${ }^{\mathrm{b}}$

aDepartments of Hepatology and Gastroenterology, Charité, CCM, Berlin, Germany; 'bepartment of Cardiology and Angiology, Charité, CCM, Berlin, Germany; Institute of Radiology, Charité, CVK, Berlin, Germany; 'Departments of Hepatology and Gastroenterology, Charité, CVK, Berlin, Germany

Introduction: Carcinoid heart disease with severe valve destruction represents the major cause of high morbidity and mortality in patients with carcinoid syndrome. $\mathbf{A i m}(\mathbf{s})$ : In this case report, we present a patient suffering advanced carcinoid heart disease at whom a two-stage minimally invasive percutaneous approach for management of severe pulmonary and tricuspid valve regurgitation was applied for the first time. Materials and Methods: A patient with an ileal neuroendocrine tumour (G2, Ki67: 5\%) presented with right heart failure (NYHA IV). Echocardiography disclosed advanced carcinoid heart disease affecting both the pulmonary and the tricuspid valves. A balloon-expandable 29-mm Edwards SAPIEN XT was successfully implanted percutaneously into the pulmonary valve. Since no catheter-based techniques were available for the replacement of the native tricuspid valve, we designed a novel approach to reduce the abdominal congestion: for the first time we implanted an Edwards SAPIEN 26-mm valve into the ICV between the right atrium and the ostium of the hepatic veins, which completely prevented regurgitation into the VCI and abdominal veins. Results: After this proce- 
dure, the patient's clinical condition improved significantly, and she achieved near-normal exercise tolerance (VO2 max: $24.4 \mathrm{ml} \mathrm{O} / \mathrm{kg}$ / min, NYHA II). Conclusion: We demonstrated that percutaneous valve implantation may offer a novel, minimally invasive option in hig risk patients with severe carcinoid heart disease. Keywords: carcinoid heart disease, percutaneous.

\section{Q18 \\ Staged Percutaneous Treatment of Severe Carcinoid Heart Disease by Transfemoral Pulmonary Valve Replacement and Novel Vena Cava Valve Implantation}

\author{
Pschowski R. ${ }^{\text {, Laule M. }}{ }^{\mathrm{b}}$, Baur A. ${ }^{\mathrm{c}}$, Pape U.F. , Pavel M. ${ }^{\mathrm{d}}$, \\ Stang/ V. ${ }^{\mathrm{b}}$, Wiedenmann B. ${ }^{\mathrm{a}}$, Stang/ $K^{\mathrm{b}}$ \\ aDepartments of Hepatology and Gastroenterology, \\ Charité, CCM, Berlin, Germany; 'bepartment of \\ Cardiology and Angiology, Charité, CCM, Berlin, Germany; \\ Institute of Radiology, Charité, CVK, Berlin, Germany; \\ dDepartments of Hepatology and Gastroenterology, \\ Charité, CVK, Berlin, Germany
}

Introduction: Carcinoid heart disease with severe valve destruction represents the major cause of high morbidity and mortality in patients with carcinoid syndrome. $\mathbf{A i m}(\mathbf{s})$ : In this case report, we present a patient suffering advanced carcinoid heart disease at whom a two-stage minimally invasive percutaneous approach for management of severe pulmonary and tricuspid valve regurgitation was applied for the first time. Materials and Methods: A patient with an ileal neuroendocrine tumour (G2, Ki67: 5\%) presented with right heart failure (NYHA IV). Echocardiography disclosed advanced carcinoid heart disease affecting both the pulmonary and the tricuspid valves. A balloon-expandable 29-mm Edwards SAPIEN XT was successfully implanted percutaneously into the pulmonary valve. Since no catheter-based techniques were available for the replacement of the native tricuspid valve, we designed a novel approach to reduce the abdominal congestion: for the first time we implanted an Edwards SAPIEN 26-mm valve into the ICV between the right atrium and the ostium of the hepatic veins, which completely prevented regurgitation into the VCI and abdominal veins. Results: After this procedure, the patient's clinical condition improved significantly, and she achieved near-normal exercise tolerance (VO2 max: $24.4 \mathrm{ml} \mathrm{O} 2 / \mathrm{kg}$ / min, NYHA II). Conclusion: We demonstrated that percutaneous valve implantation may offer a novel, minimally invasive option in hig risk patients with severe carcinoid heart disease. Keywords: carcinoid heart disease, percutaneous.
Q19

\section{Targeted Therapies as First Line Option in Very Advanced Unresectable Pancreatic Neuroendocrine Tumors}

\author{
Rho Y.S., Gilabert M., McLean J., Kavan P. \\ Jewish General Hospital McGill University, Montreal, \\ Canada
}

Introduction: Neuroendocrine tumors are an entity of rare, heterogeneous group of tumors that can arise from different sites within the body. Although number of different treatment types and modalities exist, chemotherapy remains as the first line option in the management of advance pancreatic NETS (pNETS); two targeted therapies, sunitinib and everolimus remains available only for second line and beyond in Canada. Aim(s): Case series illustrating the possible use of targeted therapies in the first line treatment of advanced pNETS. Materials and Methods: We describe case of 4 chemo-naïve patients (age at diagnosis 46-71; 3 male, 1 female) with advanced pNETs. These patients were initially unresectable, deemed palliative or refused chemotherapy as first line. Targeted therapy was commenced between 2011-2012. Results: All 4 patients achieved significant disease control (18 month and beyond), while maintaining acceptable quality of life. Conclusion: This case series illustrates the complexity involved in the management advanced pNETS, stressing the need for open dialogue in an attempt to practice patient centred medicine based on best available options. In addition, it highlights the role of targeted therapies which in the future may have a much more mainstream role. Keywords: targeted therapy, sunitinib, everolimus, pnet.

\section{Q20 \\ Cowden Syndrome and Concomitant Pulmonary Neuroendocrine Tumor: A Presentation of Two Cases}

Ringholm L., Langer S., I Dali C., Petersen R.H., Rasmussen A.K., Gerdes A.M., Federspiel B., Knigge U.P.

Rigshospitalet, Copenhagen University Hospital, Copenhagen, Denmark

Introduction: Cowden Syndrome is a multiorgan disorder with germline mutations in the tumor suppressor gene PTEN leading to dysfunctional cell growth and risk of neoplasms in e.g. breast and thyroid, but no association with NET has been described. Aim(s): To present two cases. Materials and Methods: Two patients with Cowden Syndrome and pulmonary NET are presented. Results: Case 1: 55-year old woman with previous resection of a follicular thyroid adenoma and bilateral mastectomy due to invasive ductal breast carcinomas. FDG PET/CT revealed lung tumors, which were resected thoracoscopically. Immunohistochemical staining showed typical carcinoids positive for chromogranin and synaptophysin. Breast carcinoma markers were negative. Mitotic count was $<2 / 2$ $\mathrm{mm}^{2}$. Tumor necrosis was absent. Plasma chromogranin A was normal postoperatively. Case 2: 61-year old man with Lhermitte-Duclos 
disease, previous resection of a follicular thyroid carcinoma and skin melanoma. 68Galium-DOTATOC PET/CT revealed moderate uptake at the left lung hilus and metastases in lymph nodes, liver and bones. Fine needle aspiration of a lymph node revealed an atypical carcinoid tumor positive for chromogranin and synaptophysin. Mitotic count was $2 / 2 \mathrm{~mm}^{2}$. Tumor necrosis was absent. Plasma chromogranin A was elevated 3-fold. Conclusion: Long-term follow-up is warranted to establish if patients with Cowden Syndrome are at increased risk of NET. In the meantime, we recommend annual chromogranin A measurements to screen for NET in these patients. Keywords: cowden syndrome, pulmonary net.

\section{Q21 \\ Optimal Clinical Effect of Sandostatin LAR Treatment for Gastroenteropancreatic (GEP) Carcinoid}

Robev B., Sergieva S.

Sofia Cancer Center, Sofia, Bulgaria

Introduction: The incidence of carcinoid is $2 / 100000$ with a consistent trend grow due to improved diagnosis of these tumors in recent years. There are two peaks of carcinoid incidence - the first between the 15th and 25th years and the second-between the 65th and 75th years. Aim(s): This clinical case is about 65 year old lady with carcinoid of ileum operated in 1990 stage T2N0M0. Omental and hepatal metastases of the same tumor with increased level of Chromogranin A and expressed flush syndrome were diagnosed in this patient 10 years later. Materials and Methods: Surgery treatment was not considered in this patient due to disease extension, therapy with Sandostatin LAR was indicated and was performed since 1999. Treatment monitoring was carried out by CT studies, serum level of Chromogranin A assessment, and recently using SPECT-CT somatostatin-receptor scintigraphy. Results: The patient was treated with Sandostatin LAR in dose $20 \mathrm{mg} / 28 \mathrm{~d}$ from 1999th to 2011th, when the disease progression was established. Patient treatment continued with increased dose of Sandostatin LAR-30 mg/28 d. In this case we observed 13-year progression free survival and successfully controlled disease for 2 years and 5 months with higher dose of Sandostatin LAR. Conclusion: This case report demonstrates the effective management of a patient with GEP carcionoid with Sandostatin LAR to enable optimal outcome and a significant prolongation of overall survival with an efficiently control the disease symptoms. Keywords: carcinoid, sandostatin lar.

\section{Q22 \\ Malignant Insulinoma in a Patient with Diabetes: Response to Treatment and a Return to Insulin Injections}

Sherriff D., Drake $B$.

Derriford Hospital, Plymouth, UK

Introduction: A 64 year old male with a fourteen year history of diabetes had been treated with insulin for eight years. Over a four week period he noticed that his insulin requirements were reducing and he experienced episodes of symptomatic hypoglycaemia and upper abdominal pain. Following referral a CT scan was performed which revealed numerous liver metastases and a 8.4 by $5.2 \mathrm{~cm}$ mass replacing the pancreas. Liver biopsy diagnosed a neuroendocrine tumour with a MIB-1 index of up to $18 \%$. The tumour was somatostatin receptor positive as evidenced by an octreotide scan. Aim(s): An effective treatment was required as Everolimus and chemotherapy were not successful. Materials and Methods: Following discontinuation of Everolimus due to toxicity and unsuccessful treatment with chemotherapy (Streptozocin and Capecitabine) the patient was treated with PRRT (Peptide Receptor Radionuclide Therapy). He received 7,400 MBq Lu177 DOTATATE as a single treatment. Results: Whole body nuclear medicine imaging demonstrated extensive tracer uptake in the known disease. Two days following treatment the glucose infusion was discontinued and the patient required insulin again. Conclusion: Insulinoma is a rare pancreatic neuroendocrine tumour which can present with severe hypoglycaemia. Peptide Receptor Radionuclide Therapy has been demonstrated to be very successful in controlling this. Interestingly, our patient with preexisting diabetes required the reintroduction of insulin following a single treatment with Lu177 DOTATATE. Keywords: insulinoma, diabetes, prrt.

\section{Q23 \\ Antiproliferative Effect of Lanreotide in Low Grade Neuroendocrine Neoplasms: Two Case Reports}

Vaslamatzis M., Tegos T., Alevizopoulos N., Stathopoulos C., Argyrakos T., Kapou T., Patila E., Vlontzou E., loannidou S., Rontogianni D.

Evangelismos Hospital, Athens, Greece

Introduction: Somatostatin analogs are used for control of symptoms due to hormonal hypersecretion by neuroendocrine neoplasms (NEN). Their antiproliferative effect only recently became evident. Data on pancreatic NEN (pNEN) are limited. Aim(s): We present patients with $\mathrm{pNEN}$ that received Lanreotide. Materials and Methods: 2 case reports. Results: CASE 1: Male 78 yo, weight loss (6 kg), diarrhea (8-10/d), body rash (last 3/12). ECOG/PS: 3, CgA: $433 \mathrm{mmol} / \mathrm{l}, 5$-HIAA $903 \mathrm{mg} / 24 \mathrm{~h}$, NSE $27 \mathrm{ng} / \mathrm{ml}$, VIP 7 pmol/1, gastrin $15 \mathrm{pg} / \mathrm{ml}$. Octreoscan: lesion at the body of the pancreas, multiple liver lesions ( $\max 10 \mathrm{~cm}$ by $\mathrm{U} / \mathrm{S}$ ), lesions in thoracic spine and femur and cervical area. Liver biopsy: pNEN metastasis, grade 1, 
Ki-67 $<1 \%$, absent mitoses. Treatment was started with Lanreotide gel $120 \mathrm{mg} / 28 \mathrm{~d}$. Diarrhea and lab tests improved, rash disappeared, weight gain, ECOG/PS: 1-2. Liver lesions diminished (max $6.3 \mathrm{~cm}$ ). Grade $\geq 3$ AE: none. PFS: 10 months. CASE 2: Female 43 yo, epigastric pain, weight loss (4 kg), ECOG/PS: 1 . Lab tests: CgA $17 \mathrm{mmol} / 1$, 5-HIAA $7 \mathrm{mg} / 24 \mathrm{~h}$, NSE $15 \mathrm{ng} / \mathrm{ml}$, gastrin: $98 \mathrm{pg} / \mathrm{ml}$. Octreoscan: lesion at the tail of the pancreas, multiple liver lesions ( $\max 8.2 \mathrm{~cm}$ by $\mathrm{U} / \mathrm{S}$ ). Liver biopsy: pNEN metastasis, grade 1 , Ki-67 $<3 \%$, mitoses 1-2/10 hpf. Treatment was started with Lanreotide gel $120 \mathrm{mg} / 28 \mathrm{~d}$. Liver lesions diminished in size after 3/12. Grade $\geq 3$ AE: none. PFS: 6 months. Conclusion: In conclusion, first line treatment with Lanreotide $120 \mathrm{mg}$ in patients with grade $1 \mathrm{pNEN}$ had an antiproliferative effect without toxicity and warrants further study. Keywords: nen, lanreotide.

\section{Q24 \\ Salvage Treatment with Peptide Receptor Radionuclide Therapy (PRRT) in Patients with Advanced VIPomas and Severe Verner-Morrison Syndrome}

\author{
Yalchin M. ${ }^{a}$, Gertner J. ${ }^{a}$, Navalkissoor S. ${ }^{b}$, Quigley A.M. ${ }^{b}$, \\ Meyer T. ${ }^{\mathrm{c}}$, Caplin M. ${ }^{\mathrm{a}}$, Toumpanakis $\mathrm{C}^{\text {a }}$ \\ aNeuroendocrine Tumour Unit, ENETS Centre of \\ Excellence, Royal Free Hospital, London, UK; bNuclear \\ Medicine Department, Royal Free Hospital, London, UK; \\ 'Oncology Department, Royal Free Hospital, London, UK
}

Introduction: PRRT is an effective treatment option for advanced Neuroendocrine Tumours. Aim(s): Demonstrate its salvage role in 2 patients with advanced VIPomas with ongoing symptoms and progressive disease, despite other treatments. Materials and Methods: Two patients (63 and $59 \mathrm{yrs)} \mathrm{included.} \mathrm{Results:} \mathrm{They} \mathrm{presented} \mathrm{with}$ diarrhoea, hypokalaemia, weight loss, raised VIP and had pancreatic primaries (2nd patient already had hepatic and paraaortic LN metastases). Initial treatments [resection of primary and commencement on long-acting somatostatin analogues (SST), respectively] resulted in total symptom resolution. Tumours were well-differentiated, G2. 1 st patient developed hepatic, LN and skeletal metastases and relapse of diarrhoea, 3 years after diagnosis, and was commenced on SST. The 2nd patient had symptomatic and radiological progression, 6 yrs after diagnosis and had chemotherapy (also given to the 1st patient), because of ongoing symptoms. None of the patients responded and so, were commenced on everolimus and sunitinib, respectively, with no response. Both patients were commenced on PRRT [3 cycles Y-90 DOTATATE and 4 Lu-177 DOTATATE, respectively] which resulted in normalization of bowel movements, VIP, and radiological stabilization of their disease (48 and 24 months after the 1st cycle respectively). Conclusion: PRRT seems to be effective in controlling symptoms and tumour growth in advanced/progressing VIPomas. Studies with larger amount of patients are required to establish its earlier role in treatment of these patients. Keywords: prrt vip.

\section{Q25}

Role of Cytoreductive Surgery (CRS) and Intraoperative Hyperthermicintraperitoneal Chemotherapy (HIPEC) for High Grade Neuroendocrine Tumour

\author{
Yalchin M. ${ }^{\text {a }}$ Cecil T. ${ }^{\mathrm{b}}$, Eldeep H. ${ }^{\mathrm{c}}$, Toumpanakis C. ${ }^{\mathrm{a}}$, \\ Caplin M. ${ }^{a}$ \\ ${ }^{a}$ Neuroendocrine Tumour Unit, ENETS Centre \\ of Excellence, Royal Free Hospital, London, UK; \\ bBasingstoke Colorectal Unit, Hampshire Clinic Basing \\ Road, Basingstoke, UK; ' Oncology Department, Milton \\ Keynes Hospital, Milton Keynes, UK
}

Introduction: Managing patients with omental and peritoneal Nueroendocrine tumour is challenging. $\operatorname{Aim}(\mathbf{s})$ : Demonstrate role of cytoreductive surgery and intraoperative chemotherapy in selected patients. Materials and Methods: 1 patient (42 year old male) included. Results: Presented in December 2013 with 5 days lower abdominal pain and fever, but no syndromic features. Examination revealed tender mass in the left iliac fossa, performance status 0 . CT showed necrotic mass, $15 \mathrm{~cm}$ in left iliac fossa arising from the small bowel, with nodules present in the omentum and peritoneum. US guided biopsy of mesenteric mass showed poorly differentiated grade 3 NET, Ki67 35\%. FDG PET scan highlighted ileal primary, with multiple omental/peritoneal metastases with high SUV. Tumours only mildly avid on Gallium 68 octreotate PET. There was no other disease. Patient received 6 cycles of Etoposide and Cisplatin chemotherapy between February-May 2014 with partial response. In view of his young age, performance status, and 'limited disease' we referred him to the specialist Surgical Unit at the Basingstoke in June 2014. He had excision of omentum, transverse colon, small bowel mass, plus appendicectomy and peritectomy. Intraoperative Cisplatin and Doxorubicin was given at $41 \mathrm{C}$ Histology of the mass showed NEC, Ki67 50\%. Post operative FDG PET CT and contrast CT scan demonstrated no evidence of residual disease. Conclusion: CRS and HIPEC have been used successfully in selected cancers and this case suggests a possible role in intra-abdominal NET. Keywords: crs, hipec, net. 


\section{Q26}

\section{Subtype Classification and Clinicopathological Characteristics of 78 Cases with Gastric Neuroendocrine Neoplasms}

\author{
Zhang C. ${ }^{a}$, Tan H.Y.a, Liu J.X. ${ }^{\mathrm{b}}$, Luo J. ${ }^{\mathrm{c}}$, Yu L.L. ${ }^{\mathrm{a}}$, Fang L. ${ }^{\mathrm{b}}$, \\ Wang M. ${ }^{\text {, }}$, Deng B. ${ }^{\text {a }}$, Jia L. Q. ${ }^{\text {a }}$ \\ aDepartment of Intergrative Oncology, China-Japan \\ Friendship Hospital, Beijing, China; 'bepartment of \\ Gastroenterology, China-Japan Friendship Hospital, \\ Beijing, China; 'Department of Pathology, China-Japan \\ Friendship Hospital, Beijing, China
}

Introduction: Gastric NENs are a group of neoplasms with diverse clinicopathological features. $\mathbf{A i m}(\mathbf{s})$ : To investigate the subtype classification and clinicopathological characteristics of g-NENs in Chinese pts. Materials and Methods: All g-NEN pts treated at our hospital between Jun 2012 to Oct 2014 were subtyped with the criteria: well-differentiated g-NENs classified into 3 types: type 1, with hypergastrinemia and achlorhydria, associated with autoimmune gastritis; type 2, with hypergastrinemia and ZES, associated with gastrinoma or MEN-1; type 3, sporadic disease with normal gastrin and gastric acid secretion; type 4, poorly-differentiated NEC or MANEC. The clinicopathological features were analysed. Results: A total of 78 g-NENs were studied: 37 type 1 (47.4\%), 4 type 2 (5.1\%), 26 type 3 (33.3\%) and 11 type 4 (14.1\%). Type 1 were mostly multiple and small polypoid lesions of fundus or body, with NET G1 (89.2\%), while 24 of $25 \mathrm{pts}$ receiving $24 \mathrm{~h}$ intragastric $\mathrm{pH}$ monitoring had achlorhydria. All of 37 pts had no metastasis. Type 2 mostly showed multiple and small polyps of fundus or body, 3 cases with gastrinoma and one with MEN-1. Type 3 contained NET G1 (50.0\%), NET G2 (42.3\%) and NET G3 (7.7\%) in pathologic grading. 17 pts $(65.4 \%)$ had metastases. All of type 4 had metastases at diagnosis. Conclusion: Each subtype of g-NENs has distinguished features. The serum gastrin testing and the $24 \mathrm{~h}$ intragastric $\mathrm{pH}$ monitoring are the significant methods for g-NENs subtype classification. Keywords: gastric neuroendocrine tumors, serum gastrin, $24 \mathrm{~h}$ intragastric ph monitoring.

\section{Q27}

\section{Clinicopathological Features and Prognostic} Factors of Gastric Neuroendocrine Tumors:

\section{A Retrospective Analysis of 69 Cases}

\author{
Zhao C.H., Li Y. \\ Cancer Hospital, Chinese Academy of Medical Sciences, \\ Beijing, China
}

Introduction: Gastric neuroendocrine tumors (GNETs) are rare tumors, but the incidence has increased in most countries. Aim(s): To analyze the clinicopathological features and their relationship with prognosis in GNET patients. Materials and Methods: 69 GNET patients were surgically treated and confirmed pathologically. Their clinical records were retrospectively analyzed. Results: Of the 69 patients, $29(42 \%)$ were diagnosed as type I GNETs, 33 (48\%) were diagnosed as type III, and $7(10 \%)$ were diagnosed as type IV. Of the 29 type I GNET patients, 9 underwent gastrectomy and the others were treated with EMR. 2 type III GNET patients were treated with palliative gastrectomy, 2 with exploration only due to distant metastasis. The others were treated with radical resection. All the type I GNETs were classified as G1 or G2 according to the WHO grading system. 30 type III patients were G3, 2 were G1 and 1 was G2. Type IV GNETs were all G3. There were statistically significant differences between type I and type III or IV GNETs in the sex, tumor number, location, size, serosa invasion and the lymph node metastasis ( $<<0.05)$. 3-year survival rates of type I, III, and IV GNETs were $100 \%, 49 \%$ and $67 \%$, respectively. Univariate analysis revealed tumor size $(>2 \mathrm{~cm})$, serosal invasion, lymph node metastasis, WHO grading and classificasion were all significant factors affecting the survival $(p<0.05)$. Conclusion: GNETs should be treated differently based on their subtypes. The clinicopathological features are helpful in predicting prognosis. Keywords: gnet, clinicopathological features, prognosis.

\section{Q28 \\ The Challenge of Differential Diagnosis of Solid Pancreatic Lesions in Von Hippel-Lindau. Case Report

\author{
Zilli A. ${ }^{\mathrm{a}}$, Cavalcoli Fa, Maddalena P. ${ }^{\mathrm{b}}$, Conte D. ${ }^{\mathrm{a}}$, \\ Massironi a $^{\text {a }}$ \\ ${ }^{a}$ Gastroenterology and Endoscopy Unit, Department of \\ Pathophysiology and Transplantation, Fondazione IRCCS \\ Cà Granda Ospedale Maggiore Policlinico, Milan, Italy; \\ bUniversità degli Studi di Milano, Milan, Italy
}

Introduction: Von Hippel-Lindau (VHL) is an hereditary syndrome, characterized by a predisposition to develop benign and malignant tumors. Pancreatic involvement is present in $35-70 \%$ of cases and include cystic (simple cyst, serous cystoadenoma, mucinous tumor or IPMN) and solid lesions (neuroendocrine neoplasm (NEN), metastatic mass or pancreatic adenocarcinoma). Finally, a solid-type serous cystoadenoma (SSCA) has been reported. Differential diagnosis of these lesions may be difficult. $\mathbf{A i m}(\mathbf{s})$ : To present a case of an unclear solid pancreatic lesion in a patient affected by VHL. Materials and Methods: In a 32-year-old VHL man a CT scan, performed for follow up of pheocromocytoma, detected a contrastenhanced lesion of $16 \mathrm{~mm}$ in the uncinate process without necrosis or calcifications. Results: This lesion was negative at Ga68 PET and hypercaptant at 18 FDG-PET. EUS showed two hypoechogenic solid lesions of $3 \mathrm{~mm}$ and $16 \mathrm{~mm}$, with rapid enhancement and a hypoechogenic ring, features consistent with NEN or SSCA. Clusters of acinar $\mathrm{CgA}+$ cells without atypias were evident at cytology. Pancreatic hormones were normal. Conclusion: Pancreatic lesions could be a diagnostic challenge in VHL. Differential diagnosis between NEN and SSCA may be difficult due to their rich vascolarization and dense appearance on non-functional imaging. Moreover metastatic lesion from previous pheochromocytoma or renal cell carcinoma should have been considered. Discrepancy between PET and EUS results indicated a clear-cut need for surgical approach. Keywords: solid pancreatic lesions, vhl. 
... None of the Below

\section{R1 \\ Mesenteric Ischemia Due to Neuroendocrine Tumors of the Digestive Tract}

Corcos O. a, Hentic O. a, Nuzzo A. a, Stan-luga R. ,

Becq A. ${ }^{a}$, Sauvanet A. ${ }^{a}$, Cazals-Hatem D. ${ }^{a}$, Couvelard A. ${ }^{\text {, }}$

Bouhnik Y.a , Joly F.a , Panis Y.a, Ruszniewski P.a

aeaujon Hospital, Clichy, France; 'bichat Hospital, Paris, France

Introduction: Mesenteric ischemia (MI) is a poorly known complication of neuroendocrine tumors (NETs). $\boldsymbol{A i m}(\mathbf{s})$ : To better define MI occuring in the setting of NETs. Materials and Methods: Pts $>18$ yo, digestive NETs, chronic (CMI) or acute (AMI) MI between 2001 and 2014 consecutively enrolled. Results: 13 patients included (5 men, 6 CMI, 11 AMI). NETs characteristics: ileum $(n=8) /$ pancreas $(\mathrm{n}=5)$, non functional $(\mathrm{n}=10)$, WHO grade $1(\mathrm{n}=6)$ or $2(\mathrm{n}=$ $7)$, well differentiated $(\mathrm{n}=13)$, MIB $<10 \%(\mathrm{n}=13)$, metastatic $(\mathrm{n}=$ 11). NETs treatment: surgical resection $(n=8)$, octreotide $(n=9)$, chemotherapy $(n=5)$, embolization $(n=3)$, sunitinib $(n=2)$, everolimus $(n=5)$. MI occurred $5(0-19)$ y after the diagnosis of NET. In 3 pts MI preceded NETs. In 8 pts, AMI occured in the setting of a surgical procedure and/or shock $(n=3)$. MI was a consequence of vascular compression or tumoral invasion by a tumoral lymph node $(n=5) .9$ patients required intestinal resection. One pt underwent revascularization without resection. Amongst 5 pts with CMI, a dedicated multimodal and multidisciplinary management allowed discontinuation of parenteral nutrition in 4 pts without intestinal resection. Overall survival was $92 \%$. Conclusion: Digestive NETs may lead up to MI, in the setting of surgery or as a consequence of vascular invasion. One of the challenges is to preserve both vessels and the bowel. A specialized multimodal and multidisciplinary management, focusing on intestinal viability, can result in minimal intestinal resection, with high rates of survival. Keywords: mesenteric ischemia.

\section{R2}

\section{Identification of Novel and Pathological Feature of Angiostatic Microvasculature in Liver Metastatic Pancreatic Neuroendocrine Tumors and Its Clinical Implication}

\author{
Han X. ${ }^{a}$, Hexige S. ${ }^{\text {b }}$ Lou W. ${ }^{a}$ \\ aDepartment of General Surgery, Zhongshan Hospital, \\ Fudan University, Shanghai, China; 'btate Key Laboratory \\ of Genetic Engineering, School of Life Sciences, Fudan \\ University, Shanghai, China
}

Introduction: Microvasculature of liver metastatic pancreatic neuroendrocrine tumors (LM-PNETs) are unknown. Aim(s): To visualize in $3 \mathrm{D}$ the neoplastic associated neovasculature in
LM-PNETs and reveal its novel features. Materials and Methods: An adapted thick human tumor sections immunostaining and 3D construction imaging method was performed. Results: We identified an undiscovered distinctive feature of the mature and angiostatic microvasculature in LM-PNETs, which had long, intensive, hairy-like projections on the luminal surface of the basal membrane of microvessels that we refer to as 'basal microvilli' or 'basal endocilia'. Functionally, these basal microvilli extending from LM-PNETs microvasculature had low levels of VEGFR2 expression and activation. In addition, microvessels with decreased VEGFR2 expression and activation had more intensive and longer basal microvilli and they appeared in an obvious negative correlation. Besides, western blot and RT-PCR results also revealed that LM-PNETs tissue had decreased phosphoVEGFR2 expression compared with primary PNETs and normal pancreas. These evidences suggest basal microvilli from LM-PNETs may not depend on this signaling pathway for their growth and sustenance. Conclusion: In contrast with human primary PNETs, LM-PNETs contain a mature, angiostatic, neoplastic-associated neovasculature on which there are hairy-like basal microvilli with unknown property of pathological vascular projections in metabolism and metastasis. Keywords: liver metastatic pnet, microvessels, basal microvilli, vegfr2, pvegfr2.

R3

The Neuroendocrine Tumor (NET) Patient (pt) Perspective: Results from the First Global NET Pt Survey - A Collaboration between the International Neuroendocrine Cancer Alliance (INCA) and Novartis Pharmaceuticals

\author{
Leyden J. ${ }^{\text {a }}$ Sissons M. ${ }^{\mathrm{b}}$, Goldstein G. ${ }^{\mathrm{c}}$, Kolarova T. , \\ Bouvier C. ${ }^{\text {b }}$ \\ aThe Unicorn Foundation, Mosman, Australia; ${ }^{\text {'NET }}$ \\ Patient Foundation, Hockley Heath, UK; ${ }^{\mathrm{C}}$ The Carcinoid \\ Cancer Foundation, White Plains, USA; 'APOZ \& Friends, \\ Sofia, Bulgaria
}

Introduction: Despite a rising incidence of NETs, few studies document the NET pt experience. We present data on quality of life (QoL) and the global NET pt perspective. Aim(s): To raise awareness of the NET-related burden and share pts' perspectives on NET, medical team (MT) interactions, and educational needs. Materials and Methods: Anonymous survey of NET pts from $>12$ countries conducted by INCA/Novartis in 2014 (Americas, Asia, Europe, Oceania) on the NET pt experience, including disease impact, MT interactions, knowledge/awareness, and information needs. This NET patient survey was a collaboration between INCA and Novartis and the survey was funded by Novartis. Results: Of 1928 respondents, $71 \%$ reported a moderate to significant negative impact of NETs on QoL. Pts felt well supported by their MT, especially nurses/nurse practitioners (NPs) $(78 \%)$, but only $28 \%$ listed nurses/NPs as part of their MT vs. 70\% for oncologists. Pts made several lifestyle/work changes following diagnosis and 54\% felt only somewhat or not at all knowledgeable about NETs. Information on treatment side effects $(80 \%)$, appropriate management $(78 \%)$, and resources for talking 
with MTs (78\%) was seen as most useful. Key improvements to help pts live with NETs included expanded access to NET MT/treatments $(43 \% / 46 \%)$ and increased NETs awareness (42\%). The majority of pts $(86 \%)$ received NET-related information from NET websites and most $(60 \%)$ desired clearer knowledge of NETs long-term impact. Conclusion: This NET pt survey showed a substantial NET-related burden and identified improvements to enhance pt care, including NET MT/treatment access. Keywords: quality of life.

\section{R4 \\ Health-Related Quality of Life in Patients after Diagnosis of Nonfunctioning Asymptomatic Pancreatic Neuroendocrine Neoplasms (PNEN) $\leq \mathbf{2} \mathbf{~ c m}$}

Maurizi A. ${ }^{a}$, Partelli S. ${ }^{a}$, Pezzilli R. ${ }^{\mathrm{b}}$, Crippa S. ${ }^{\mathrm{a}}$, Falconi M. ${ }^{\mathrm{a}}$

aUniversity Hospital, Pancreatic Surgery Unit, Ancona, Italy; ' Sant'Orsola-Malpighi Hospital, Bologna, Italy

Introduction: Pancreatic neuroendocrine neoplasms (pNEN) $\leq 2 \mathrm{~cm}$ can be treated conservatively. Aim(s): To evaluate the physical, mental and psychological status of patients with non-functioning, asymptomatic pNEN $\leq 2 \mathrm{~cm}$, who underwent wait and see follow-up protocol as compared to the quality of life in the general Italian population. Materials and Methods: 31 patients with asymptomatic pNEN $\leq 2 \mathrm{~cm}$ who underwent wait and see follow-up protocol were included. All these patients were invited to participate to a survey consisting of 2 standardized health-related quality of life (HRQL) measures, which were the Italian version of the SF-12 Questionnaire and the General Health Questionnaire. Results: There were 17 males and 14 females with a median age of 65 years. At a median follow-up of 14 months, all patients were alive without evidence of disease progression. Regarding the SF-12 questionnaire, the physical component scores (PCS) and the mental component scores (MCS) were $50.0 \pm$ 8.2 and $47.0 \pm 10.5$, that were no statistically different from those of reference Italian population. Regarding the psychological status, 6 subjects $(19.4 \%)$ had a similar PCS-12 score whereas the MCS$12(36.1 \pm 8.5)$ was significantly lower $(\mathrm{P}=0.003)$ as compared to the 25 subjects no non-psychotic-psychiatric problems $(49.6 \pm 9.3)$. Conclusion: HRQL scores of patients with non-functioning, asymptomatic pNEN $\leq 2 \mathrm{~cm}$ were similar to the general population's and a small fraction presented psychological disorders possibly related to the presence of the neoplasm. Keywords: pnen, quality of life.

\section{R5 \\ Appendiceal Neuroendocrine Tumor: Correlation between Histopathological Factors}

\author{
Nada H., Anania M., Luong T., Garcia-Hernandez J., \\ Caplin M., Tumpanakis C., Varcada $M$. \\ Royal Free Hospital, London, UK
}

Introduction: Neuroendocrine tumours (NET) of the appendix are rare histopathological neoplasm's detected following operation for appendicitis. Clinical decisions focuses on different pathological factors for further management. With the aim of defining contemporary patterns of care in surgical practice, a critical review of the records is carried to establish a correlation between different histopathological parameters. Aim(s): to establish a correlation between pathological different pathological factors. Materials and Methods: Review of the records of all patients referred to the neuroendocrine tumour unit and pathology department at Royal free Hospital identified 199 patients with a confirmed histological diagnosis of appendix NET lesions from January 2004 to July 2014. Results: All 199 patients treated for acute appendicitis One hundred and sixteen underwent appendicectomy while 83 had colectomy operations. Tumours were located at the appendix tip in $39.2 \%$ of cases, while $34.7 \%$ in the body. Seventy $(70 \%)$ of tumour were low grade and the majority were T2 (29\%) tumours. Correlation was found between both the stage of the tumour, T-stage and N-stage, and the anatomical site of the tumour. Conclusion: This study highlights a correlation between anatomical site of the tumour and the histopathological factors in patients with NET tumours of the appendix. Consensus guidelines should ideally be developed based on histopathological factors for post surgical management. Keywords: appendicectomy, appendix, carcinoid tumour(s), neuroendocrine tumors.

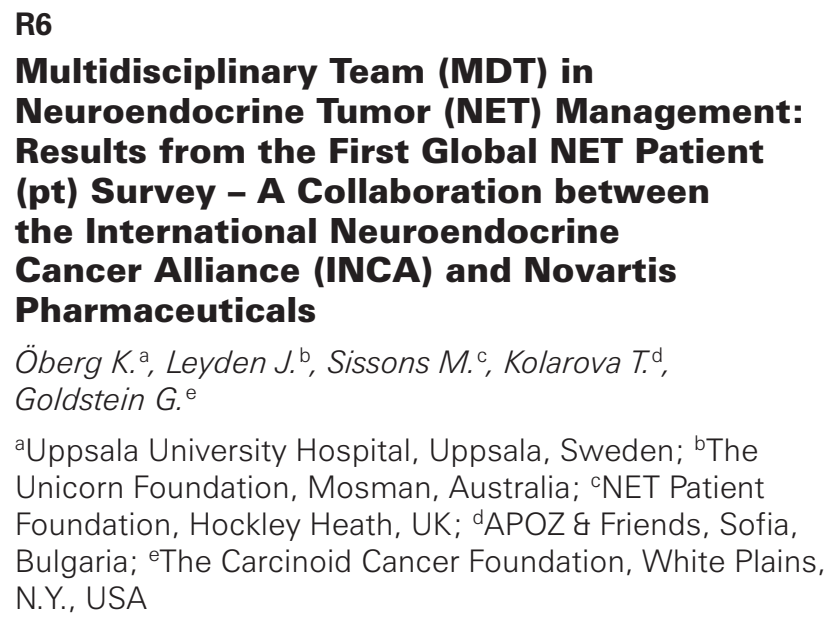

Introduction: Guidelines support an MDT approach to NETs. We present data on the global NET pt perspective regarding MDT care. $\operatorname{Aim}(\mathbf{s})$ : To assess the pt perspective on NET management and interactions with healthcare professionals (HCPs). Materials and 
Methods: Anonymous survey of NET pts from $>12$ countries conducted by INCA/Novartis in 2014 (Americas, Asia, Europe, Oceania) on the NET pt experience, including NET management and interactions with HCPs. All comparisons are significant at the $\mathrm{P}<0.05$ level. This NET patient survey was a collaboration between INCA and Novartis and the survey was funded by Novartis. Results: 1928 NET pts responded. Pts saw a median of 3 different HCPs for ongoing NET care. Pts who visited a NET specialist center (sc) $\geq 1 \times /$ year $(53 \%)$ saw a wider range of HCPs, had more tests/year (median 4 vs. 3), and discussed a broader range of NET-related topics vs. non-visitors. Compared with non-visitors, sc visitors reported better HCP relationships, $60 \%$ felt their HCPs functioned as a well-coordinated team (vs. 36\%), and 57\% wanted to work in partnership with their medical team (vs. 48\%). More sc visitors vs. non-visitors rated NET treatments quality good/very good ( $66 \%$ vs. $46 \%$ ), had access to a wider range of treatments, and were more likely to believe they received the best possible care ( $85 \%$ vs. $67 \%)$. Pt feelings regarding NETs also improved with sc visits: less frustrated/isolated $(26 \% / 15 \%$ vs. $30 \% / 22 \%)$ and more optimistic/motivated ( $24 \% / 20 \%$ vs. $18 \% / 15 \%)$. Conclusion: This NET pt survey demonstrated the benefits of an MDT, including improved satisfaction with care and interactions with HCPs. Keywords: multidisciplinary team.

\section{R7 \\ The Chicken Chorioallantoic Membrane Assay as a Preclinical Model for the Research of Rare Gastrointenstinal Neuroendocrine Tumours}

\author{
Passegger C. a, Kump P. , Haybäck J. c, Lipp R. , \\ Niederle B. ${ }^{\text {, }}$ Pfragner R. ${ }^{\dagger}$, Sadjak A. ${ }^{\dagger}$, Schwach G. ${ }^{\dagger}$, \\ Spindelböck W. ${ }^{\mathrm{b}}$, Ghaffari Tabrizi-Wizsy N. ${ }^{\text {a }}$ \\ anstitute of Pathophysiology and Immunology, SFL \\ Chicken CAM Lab, Medical University of Graz, Graz, \\ Austria; bDivision of Gastroenterology and Hepatology, \\ Department of Internal Medicine, MUG, Graz, Austria; \\ 'Department of Neuropathology, Institute of Pathology, \\ MUG, Graz, Austria; dDivision of Nuclear Medicine, \\ Department of Radiology, MUG, Graz, Austria; 'Section \\ of Endocrine Surgery, Department of Surgery, Medical \\ University Vienna, Vienna, Austria; ${ }^{\mathrm{f}}$ Institute of \\ Pathophysiology and Immunology, MUG, Graz, Austria
}

Introduction: Preclinical trials of cancer therapeutics require both in vitro and in vivo evaluations. For neuroendocrine tumours (NET) studies are limited to cell lines, thus xenograft models are needed for screening and evaluation of anti-cancer drugs. The chick chorioallantoic membrane (CAM) assay is a well-established model system to investigate tumour growth, angiogenesis and metastasis. $\operatorname{Aim}(\mathbf{s})$ : We present CAM as a suitable model for in vivo studying of gastrointestinal neuroendocrine tumours (GI-NET). Materials and Methods: Two small intestine GI-NET cell lines (P-STS and KRJ-I) and 4 patient derived GI-NET tumour tissues were grafted on CAM for $72-120$ hours at $37^{\circ} \mathrm{C}$. Xenografts were observed by light microscopy and tissues were stained for NET specific markers as chromogranin A and synaptophysin. Mitogenic activity was assessed by Ki-67.
Results: After 72 hours NET xenografts formed macroscopically visible tumours and became well vascularised. The GI-NET cell lines were mitogenic active; grew invasive within the CAM and remained to express NET specific markers. The patient derived xenografts were also viable on CAM and the immunohistochemical characteristics of the original tumour tissues were maintained. Conclusion: Our preliminary results suggest the CAM assay as a promising in vivo tool to investigate biological behaviour and tumor growth of GI-NETs. Keywords: gastrointestinal neuroendocrine tumour, chicken chorioallantoic membrane assay, patient derived tumour xenografts.

R8

Time to Diagnosis of Neuroendocrine Tumors (NETs): Results from the First Global NET Patient (pt) Survey - A Collaboration between the International Neuroendocrine Cancer Alliance (INCA) and Novartis Pharmaceuticals

\author{
Pavel M. ${ }^{\mathrm{a}}$, Goldstein G. ${ }^{\mathrm{b}}$, Sissons M. ${ }^{\mathrm{c}}$, Kolarova T. ${ }^{\mathrm{d}}$, \\ Leyden J. ${ }^{\mathrm{e}}$ \\ ${ }^{a}$ Charité Universitätsmedizin Berlin, Berlin, Germany; ${ }^{\text {b}}$ The \\ Carcinoid Cancer Foundation, White Plains, N.Y., USA; \\ cNET Patient Foundation, Hockley Heath, UK; dAPOZ

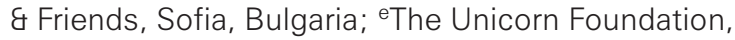 \\ Mosman, Australia
}

Introduction: Diagnosis of NETs often occurs late in the disease course, which may have an impact on pt survival. We present pt-reported data on time to diagnosis in global NET pts. Aim(s): To raise awareness of the NET pt perspective and challenges faced while seeking a diagnosis. Materials and Methods: Anonymous survey of 1928 NET pts from $>12$ countries conducted by INCA Novartis in 2014 (Americas, Asia, Europe, Oceania) on the NET pt experience, including diagnosis. This NET patient survey was a collaboration between INCA and Novartis and the survey was funded by Novartis. Results: $60 \%$ of pts reported being diagnosed $<5$ years ago; median time from symptom onset to NET diagnosis was 24 months. While time to diagnosis has improved by 10 months in pts diagnosed $<5$ years ago vs. $\geq 5$ years ago, $29 \%$ of pts still required $\geq 5$ years for a NET diagnosis and $58 \%$ of pts had metastatic disease at diagnosis. Pts saw a median of 4 health care professionals (HCPs), with a median of $6 \mathrm{HCP}$ visits prior to a NET diagnosis. Few pts (18\%) were diagnosed at a NET specialist center. For almost half of the pts (45\%), NET was not the initial diagnosis; the most common early diagnosis was digestive disorders. A quarter of the pts reported being diagnosed during testing for another condition. Most pts (76\%) did not suspect their symptoms were cancer related. $71 \%$ of pts felt there was a lot of room for improvement in NET diagnosis. Conclusion: This pt survey demonstrated a substantial delay in NET diagnosis, consistent with findings in the literature, and identified areas for improvement in the NET diagnostic process. Keywords: diagnosis. 
R9

\section{Neuroendocrine Tumor (NET) Patient (pt) Experience and Disease Burden: Results from the First Global NET Pt Survey - A Collaboration between the International Neuroendocrine Cancer Alliance (INCA) and Novartis Pharmaceuticals}

\author{
Ruszniewski P. ${ }^{\mathrm{a}}$ Yao J.C. ${ }^{\mathrm{b}}$, Kolarova T. ${ }^{\mathrm{C}}$, Goldstein G. ${ }^{\mathrm{d}}$, \\ Sissons M. ${ }^{e}$, Leyden J. ${ }^{\dagger}$ \\ aUniversity of Paris VII and Beaujon Hospital, Paris, France; \\ bThe University of Texas MD Anderson Cancer Center, \\ Houston, Tex., USA; ' APOZ \& Friends, Sofia, Bulgaria; \\ dThe Carcinoid Cancer Foundation, White Plains, N.Y., \\ USA; eNET Patient Foundation, Hockley Heath, UK; ${ }^{\text {fThe }}$ \\ Unicorn Foundation, Mosman, Australia
}

Introduction: Global data on impact of NETs on pts' lives, including differences by disease location, are poorly described. We present global data on pt experience and disease burden by NET type. $\operatorname{Aim}(\mathbf{s})$ : To raise awareness of the burden of NETs on pts' daily lives by tumor location. Materials and Methods: Anonymous survey of NET pts from $>12$ countries conducted by INCA/Novartis in 2014 (Americas, Asia, Europe, Oceania) on the NET pt experience, including impact of NETs on pts' lives. All comparisons are significant at the $\mathrm{P}<0.05$ level. This NET patient survey was a collaboration between INCA and Novartis and the survey was funded by Novartis. Results: Most pts reported having gastrointestinal (GI) NETs (1045/1928; $54 \%)$, followed by pancreatic NETs (pNETs) $(22 \%)$ and lung NETs $(12 \%)$. More pts with GI (73\%) than lung NETs (66\%) reported a negative impact on daily life. Most pts experienced NET-related symptoms daily, often associated with NET location. Breathlessness/ wheezing occurred more with lung (59\%) than GI $(25 \%)$ or pNETs $(13 \%)$. Diarrhea occurred more with GI $(60 \%)$ than pNETs $(37 \%)$ or lung NETs $(25 \%)$. Pts with GI $(62 \%)$ or pNETs $(59 \%)$ more often made dietary changes than lung NET pts (39\%). Likewise, more pts with GI $(84 \%)$ or pNETs $(83 \%)$ stopped working due to NETs than lung NET pts (69\%). Of key improvements to live better with NETs, lung NET pts were more likely to desire better access to NET-specific treatments/medical team (MT) and a more knowledgeable MT. Conclusion: This NET pt survey demonstrated a substantial impact of NETs on pts' daily lives across tumor types and identified improvements to enhance pt care. Keywords: symptom burden.
R10

\section{Epirubicin Loaded Polyphosphazene; A New Agent against Small Intestinal Neuroendocrine Tumors and Medullary Thyroid Carcinomas}

\author{
Schwach G. ${ }^{a}$, Pfragner R. ${ }^{a}$, Hoeger H. ${ }^{b}$, Schoefberger W. \\ Wilfert S. ${ }^{\text {, Brueggemann O. }}{ }^{d}$, Teasdale I. ${ }^{\mathrm{d}}$ \\ alnstitute of Pathophysiology and Immunology, Medical \\ University of Graz, Graz, Austria; 'bivision of laboratory \\ Animal Science and Genetics Himberg, Medical University \\ Vienna, Himberg, Austria; 'Institute of Inorganic \\ Chemistry, Johannes Kepler University Linz, Linz, Ausria; \\ dInstitute of Polymer Chemistry (ICP), Johannes Kepler \\ Universität Linz, Linz, Austria
}

Introduction: Small intestinal neuroendocrine tumors (SI-NETs) represent a group of rare neoplasms, derived mainly from enterochromaffine cells. Medullary thyroid carcinoma (MTC) is a calcitonin-producing neuroendocrine tumor arising from the parafollicular C-cells. SI-NETs as well as MTCs are known for their poor response to standard therapy. Therefore, there is a substantial need to establish new therapeutic options in the clinical treatment of these tumors. Aim(s): Earlier studies have demonstrated antiproliferative and tumoristatic effects of epirubicin. In this study we investigated the effects of a novel water soluble drug carrier agent, polyphosphazene on small intestinal neuroendocrine tumors and medullary thyroid carcinomas. Materials and Methods: SI-NET and the MTC cell lines were incubated with unloaded and epirubicin loaded polyphosphazenes, and cells were analysed using cell counting and WST-1 cytotoxicity assay. Fibroblasts served as contol cells. In addition, MTC xenografts were treated with unloaded, epirubicin loaded polyphosphazene, and epirubicin intra venous. Results: Treating cells with epirubicin loaded polyphosphazene resulted in antiproliferative effects of SI-NET cell lines, KRJ-I, P-STS, L-STS, H-STS and the MTC cell lines MTC-SK and SHER-I. An inhibition of cell proliferation, as well as a decrease in cell viability was noted. Conclusion: This study provides a promising basis for further research regarding the use of polyphosphazenes in the treatment of SI-NETs and MTC. Keywords: epirubicin, polyphosphazene, neuroendocrine tumor. 


\section{R11}

\section{Usefulness of a Combination of a 48-Hour Fasting and a Glucagon Tolerance Test}

Ueda K. ${ }^{a}$, Lee L. ${ }^{a}$, Hijioka M. ${ }^{a}$, Igarashi H. ${ }^{a}$, Ito T. , Jensen R.T. ${ }^{\mathrm{b}}$

aDepartment of Medicine and Bioregulatory Science, Graduate School of Medical Sciences, Kyushu University, Fukuoka, Japan; 'bigestive Diseases Branch, National Institutes of Diabetes, Digestive and Kidney Diseases, National Institutes of Health, Bethesda, Md., USA

Introduction: The 72-hour fasting test is the gold standard for the diagnosis of insulinoma. However, the test can become a burden for both patients and medical staff and, shortening the duration of fast has been attempted. There are several reports on the high sensitivity of fasting test but only a few reports on its specificity. An insulinoma can be localized using invasive tests such as endoscopic ultrasonography and selective arterial secretagogue injection test. Therefore, insulinoma detection with high specificity may be needed to avoid unnecessary examinations. Aim(s): The aim of this study is to evaluate the effectiveness of a combination of a 48-hour fasting and a glucagon tolerance test. Materials and Methods: Data were retrospectively collected from the inpatients who were suspected insulinoma between September 1992 and July 2014. These patients underwent a 48-hour fasting test followed by a glucagon tolerance test. Results: We identified 15 patients with insulinoma confirmed histologically. Insulinoma was excluded in 20 patients. The sensitivity and specificity of the 48-hour fasting test alone and glucagon tolerance test alone were $100 \%$ and $80 \%$, and $93.3 \%$ and $75 \%$, respectively. When the patients with positive results for both tests were tested using the 48-hour fasting glucagon tolerance test, the sensitivity and specificity were $93.3 \%$ and $95 \%$, respectively. Conclusion: The 48 -hour fasting test combined with a glucagon tolerance test was effective in detecting insulinoma. Keywords: insulinoma, fasting test, glucagon tolerance test. 
Aagnes B. D3

Abbassi R. C5

Abu-Hilal M. O8

Adesanya O. K11

Airoldi M. L4

Alaggio R. E8, Q10, Q12

Alaimo D. H1, H6, H12

Albarello L. G7

Albers M. E12

Alevizopoulos N. Q23

Alexandraki K. F1, H7

Aller J. L7, L8

Al-Nahhas A. Q4, Q5

Alonso T. L7, L8

Alonso-Orduña V. L7, L8

Álvarez-Escolá C. L7, L8

Alwani R.A. E5

Amadori D. J2

Ambrosini V. H5

Ambrosio M.R. C3

Anania M. R5

Anderco D. G5

Andriulli A. B1

Anlauf M. A7

Antonodimitrakis P. N1

Antonuzzo A. J10

Antonuzzo L. J11

Antwi K. I15

Apostolidis L. J1, L1

Argyrakos T. Q23

Arion A. D4

Armstrong T. E10, O8

Arndt C. Q6

Arsenic R. A7, E3, H11

Aslani A. I1

Aslanian H. H12

Atlan J. O2

Atlan K. O2

Atlan P. K9

Atreya C. L5

Aubert A. I11

Avniel-Polak S. A1

Bacher M. J9, L2, L13

Baete K. I18

Bai C.M. B5, B7

Bailey D. I1

Balboni F. A6, C1

Barata P. E9

Baratelli C. Q1

Barberis M. G7, J11
Bardan R. G5

Barriuso J. L7, L8

Bartsch D. E12, O11

Bassi C. I9, I10, O5, O6

Basuroy R. G8, H2, H14, O10

Batarda L. E9

Baudin E. J12, L9, P4, P6

Baum R. H5, H6

Baur A. I2, Q17, Q18

Beck S. B2

Becq A. R1

Bednarek-Papierska L. H10

Bedossa P. G2, L6

Begum N. J9, L2, L13, O1, O11

Beichler C. B4

Belfiori G. O12

Belletrutti P. I17

Belli S. F6, H3, Q2

Bellio M. C1

Bellucci M.C. I7

Benfini K. A2, A3, A4, A6, C3

Bennink R. I5

Benslama N. L3

Benten D. C6

Berardi R. H4

Berdelou A. L9

Bergestuen D.S. D3

Bergsland E. L5

Bestani C. F6, H3, N2, Q2

Bianchi A. Q8, Q15

Biasco E. J10

Biermann K. M3

Birch E. N4

Birocco N. L4

Bisschop P. E4

Bitossi R. Q1

Black M. H16

Blank A. G1

Blankenstein O. I12

Blau L. O2

Bock E. B4

Bockhorn M. C6

Bodei L. H1, H5, H6, H12

Bogaerts K. I18

Boggi U. B1

Boige V. L9

Bollard J. A13, L3

Bols A. D1

Bongiovanni A. J2, N6

Boninsegna L. O6

Boos L. G1
Borbath I. D1, L10

Borget I. L9, P4

Boudreaux J. O15

Bouhnik Y. R1

Bouki A.E. F1

Bouledrak K. J12, P6

Bouma G. A11, M1

Boutzios G. F1, H7

Bouvier C. D2, M2, R3

Boyar Cetinkaya R. D3

Brabant G. O1

Brandt U. L16

Brelsford M. H16

Briest F. A5

Brizzi M.P. L4, Q1

Brueggemann O. R10

Brugnoli V. J4

Bryanton M. N5

Bucau M. G2

Büchler M.W. B1, O9

Bugalho M.J. F7

Buil-Bruna N. K1

Buonomano P. K7

Burge M. N3

Bürk C. O1

Burtin P. L9

Busch O. E4, I5, O3

Busse A. H11

Butturini G. E2, I9, I10, O5, O6

Cabanne A. Q2

Cadiot G. K2, K3, K4, Q6

Callahan S. H1, H6, H12

Calvo-Temprano D. L14

Cambiaghi V. A14

Camera L. K8

Cameron S. H8

Campa D. B1

Campana D. F5

Campos T. L5

Caneva E. A6

Canzian F. B1

Cao K. I6

Capatina C. H9

Capdevila J. K2, K3, K4, L7, L8, L16

Capella C.R. G7

Capelli P. I9, O5, O6

Caplin M. B2, B6, E12, F9, K2, K3, K4, K6, K9, K10, L15, M6, O11, Q24, Q25, R5
Caplyn M. O14

Capoccacia R. D7

Capurso G. B1, F5, I8, I13

Caramanti M. H4

Caramella C. P4

Carmona-Bayonas A. E1, J6, L7, L8, L14

Carnaghi C. A14, G7

Caroli P. N7

Carratù A.C. K7

Carsote M. H9

Cascinu S. H4

Cassier P. J3

Castaño A. E1, J6

Catarino A.L. F7

Cavalcoli F. Q3, Q28

Cavaliere D. J2

Cave J. E10, O8

Cazals-Hatem D. R1

Cecil T. Q25

Cella C. J11

Chakrabarty B. E6

Chambers L. K11

Chardon L. H15

Chatzellis E. F1, H7

Chen H.E. J5

Chen J. B5, B7

Chen Y.J. B5, B7

Chen Y.L. J5

Chen Z. O4

Chiloiro S. Q15

Christ E. I15

Christen F. A5

Christensen C.U. F2

Chrysochoou M. F1

Chua Y.J. F4

Ciafardini C. E7, Q3

Cicchese N. F5, I8, I13

Cingarlini S. E2, I9, I10, O5, O6

Cipri C. F3

Circelli L. A12

Cirocchi R. O12

Ciuffreda L. L4

Cives M. G3, H16, L5

Clarke S. I1

Claro I. F7

Clement P. I18

Clift A. Q4, Q5

Colao A. A12, K7, K8

Colombé M. A13 
Colombo P. A14

Condom E. P5

Connor H. O8

Conte D. E7, Q3, Q28

Coppola D. G3, L5

Corcos O. R1

Cordier-Bussat M. A13

Cornea R. G5

Cosaro E. E2

Costa L. E9

Cotelli F. C2

Couvelard A. G2, I11, L6, R1

Crespo G. E1, J6, L7

Crippa S. O12, R4

Cros J. G2, I11, L6

Crosara S. I10

Custodio A. L7, L8, L11

Custodio P. E9

Cwikla J.B. K2, K3, K4

Dabah A. O2

Dam G. D5

Damhuis R.A. P2

Daminescu L. G5

Darby C. K11

Davì M.V. E2, I10

Davidson B. O14

Davidson R. N5

De Angelis C. L4

De Herder W. B8, E5, L10, M3

De Krijger R. A10

De Luca di Roseto C. K7

De Luca L. K8

De Marinis L. Q8, Q15

De Martino M.C. L9

De Mestier L. Q6

De Robertis R. I10

De Vries E. A11, L16, M1

Defour L. Q6

Degli Uberti E. A2, C3, J4, A3, A4, A6, C1

Degtyarev E. L10

Dehez M. K1

Del Basso De Caro M.L. A12

Del Prete M. L9, P4

Delektorskaya V. G4

Delle Fave G. B1, F5, I13, K9, I8

Dema A. G5

Demey W. D1

Demidov L. G4

Demiri M. I3

Demolin G. D1

Den Bakker M.A. P1

Denecke T. E3, I2, I14

Deng B. Q26

Derks J. P1, P2

Deroose C. I18

Derosa L. J10

D'Errico V. N7
Desiderio J. O12

Di Costanzo F. J11

Di Iorio V. N6

Di Pasquale C. A2, A3, A4, $\mathrm{C} 3, \mathrm{~A} 6$

Dibra H. B2

Dicitore A. C2

Diebold A. O15

Diebold M.D. Q6

Dieren S. E4

Dimitriadis G.K. K11

Dimitroulopoulos D. I3

Dingemans A.M.C. P1, P2

Dogan F. B8

Domenichini E. F6, H3, Q2

Donati C. N7

D’Onofrio M. I10

Drake B. Q22

Dresse D. D1

Drozdov I. H1, H5, H6, H12, H13

Drymousis P. Q4

Ducreux M. J12, L9, P4, P6

Dumitrascu A. H9

Dussol A.S. J3, J12, P6

Ebeling-Barbier C. N1

Elami A. O2

Eldeep H. Q25

Ellard S. Q8

Emelianova G. K5

Ernst S. N5

Evaggelatou A. H7

Fabre M. I11

Faez L. E1, J6, L14

Faggiano A. A12, K7, K8, L9

Fahl M. C6

Fakirova A. I16

Falcone A. J10

Falconi M. A3, A4, E12, F5, H4, I8, O11, O12, R4

Falletta S. A2, A3, A4, A6, C3

Fang L. Q26

Fani M. I15

Fantini L. N6

Farinati F. Q12

Farnesi A. J10

Farrell W.E. B8

Fazio N. F5, G7, J11, L10

Feber A. B2

Federspiel B. Q20

Feelders R.A. E5, M3

Felce M. I11

Felder S. E3

Feliu J. L7, L8

Fendrich V. O11

Feo C. C1

Fernandes I. F7

Fernandez Arrojo S. L14
Ferolla P. I7

Ferone D. C2

Ficial M. O6

Filipe F. E9

Finocchiaro G. P4

Fleming D. M4

Forestier J. J3, O13

Fottner C. J9, L2, L13

Francia G. E2

Francis J. B2

Freitag H. A5

Friemel J. A9

Friend P. Q5

Frilling A. Q4, Q5

Fuchs R. C4

Fulford P. E6

Fumagalli C. J11

Fusai G. E12, O11

Fusai G.K. O14

Füzesi L. H8

Gabrovska P. Q8

Gachramanov A. D4

Gadot N. A13

Gagliano T. A2, A3, A4, A6, $\mathrm{C} 1, \mathrm{C} 3, \mathrm{~J} 4$

Galassi R. J2

Galdy S. J11

Galli L. J10

García Mónaco R. N2

Garcia-Carbonero R. E1, J6, L11

Garcia-Hernandez J. F9, K6, R5

Gardini A. J2

Garske-Roman U. N1

Gatta G. D7

Gaudenzi G. C2

Gazouli M. B1

Ge F.J. J5

Geboes K. D1

Gelsomino F. J11

Geng C.Y. I4

Gentilin E. A2, A3, A4, A6, C1, $\mathrm{C} 3, \mathrm{~J} 4$

Geraldo Roig L. P3

Gerdes A.M. Q20

Gerlach U. E3

Gertner J. Q24

Ghaffari Tabrizi-Wizsy N. B4, C4, R7

Ghayouri M. H16

Giampietro A. Q15

Giandomenico V. B6

Gianoncelli L. L9, P4

Giele H. Q5

Gilabert M. Q19

Gimenez-Roqueplo A.P. A10

Giordano C. K7

Glasberg S. O2

Glaser B. A1

Glinicki P. H10
Gloor B. I15

Goddard I. A13

Goichot B. H15

Goldstein A. H9

Goldstein D. F4

Goldstein G. R3, R6, R8, R9

Goma M. P5

Gomes F. E9

Gomes L. F7

Gomez-Panzani E. K1, K2, $\mathrm{K} 3$, K4

Gondolesi G. Q5

Gopalakrishnan K. K11

Gorbunova V. K5

Gouma D. E4, I5, O3

Grabowski P. A5, H11

Granberg D. N1

Grande E. L7, L8

Grant L. K10

Grass I. A5

Gray D. M2, N5

Grego E. I9

Gress T. A7, A9, C5

Griesmann H. C5

Grimaldi F. F3

Grønbæk H. D5, F2

Gross D.J. A1, O2

Grozinsky-Glasberg S. A1

Guadagno E. A12

Gudlaugsson E. D6

Guerrini R. J4

Guigay J. J12, P4, P6

Guillermo C. L8

Guixia W. B9

Haas O.A. B4

Hackert T. O9

Hamilton D. N4

Hamm B. I14

Hammel P. I11, L6

Han X. R2

Hansen S. D3

Hao C. O4

Hao J. F8

Harada Y. Q14

Hasegawa H. Q14

Hatzinikolaou M. I3

Haustermans K. I18

Haybäck J. R7

Heaton N. G8, O10

Heitman S. I17

Hendifar A.E. L11

Hendriks L.E. P2

Hennessy T. I1

Hentic O. G2, I11, L6, R1

Herbst F. L11

Hervieu V. A13, H15, J3, J12, L3, O13, P6

Hexige S. R2

Heye T. I15 
Hicks R. F4, L12

Hijioka M. R11

Hobday T. L16

Hoefnagel S. I5

Hoeger H. B4, R10

Hoffmann M. O1

Hoffmeister M. A7, A8, A9

Hofland L.J. B8, M3

Holubec L. K9

Hölzl M. C4

Hommann M. A8

Hörsch D. M4

Hortopan D. H9

Hu J.H. E10

Huang D. Q7

Hubner R. E6

Humer-Fuchs U. Q16

Hummel M. H11

I Dali C. Q20

Iacovazzo D. Q8, Q15

Iannicelli E. I8

Ianniello A. J2, N6, N7

Ibrahim T. J2, N7

Igarashi H. R11

Iliadis A. G6

Imber C. O14

Impellizzeri H. O5, O6

Inama M. O11, O14

Ioannidou S. Q23

Ishida H. Q14

Ito T. R11

Iwasaki R. Q14

Iwasaki T. Q14

Izbicki J. C6

Izzo F. A12

Jäger D. J1, L1

James P. I17

Jan H. N3

Jann H. E3

Jaynes E. E10

Jensen R.T. R11

Jeske W. H10

Jesús Garrido M. K1

Ji Y. J8, M5, O16

Jia L.Q. Q26

Jia R. J5

Jiang L.M. I4

Jilesen A. E4, I5, O3

Jiménez-Fonseca P. E1, J6, L7, L8, L14

Joehrens K. H11

Joly F. R1

Joly M.O. J3

Jonzzon S. H1

Jung K. B3

Kaemmerer D. A8, E11, H11

Kaltsas G. F1, H7
Kaltsatou M. F1

Kamp K. E5, M3

Kang W. B3

Kapou T. Q23

Karapetis C. F4

Karayannopoulou G. G6

Karpathakis A. B2

Karra E. K6

Kats-Ugurlu G. M1

Kaupp-Roberts S. M2

Kavan P. Q19

Kaye A. O15

Keck T. O1, O11

Kelley R. L5

Kema I. A11, M1

Khan M. B6

Khan S. K11

Kianmanesh R. Q6

Kidd M. H1, H5, H6, H12, H13

Kiesewetter B. J7

Kim S. B3

Kitsou E. H7

Kleinegger F. C4

Klumpen H.J. E4

Knigge U.P. Q20

Kocha W. N5

Kolarova T. R3, R6, R8, R9

Koletsa T. G6

Komarova L. D4

Koole M. I18

Koparek O. J7

Korach A. O2

Korbonits M. Q8

Kornerup L.S. D5

Krejs G. Q9

Krenning E. H5, H6

Krug S. C5

Krump C. C4

Kujaruk M. Q2

Kulke M.H. L10

Kump P. I8, Q9, R7

Kunz F. I2

Kunz T. L16

Kuru N. L12

Kuzminov A. K5

Kvaløy J.T. D6

Kwekkeboom D. H5, H6, M3

Kypreos D. I3

La Rosa S. G7

Lacheretz-Bernigaud A. A13

Lahner H. J9, L2, L13

Laidley D. N5

Lam D. L10, L16

Lamarca A. E6

Lamberts S.W.J. B8

Lambrechts G. D1

Landi S. B1

Landoni L. O5, O6
Langer S. Q20

Langley A. K2, K3, K4

Lania A. A14

Lapuerta P. M4

Laskaratos F. K10

Laule M. Q17, Q18

Laurent-Bellue A. G2

Lax S. Q16

Leboulleux S. L9

Lebtahi R. G2

Lee J. B3

Lee L. R11

Lee S. B3

Lehnert H. O1

Leibowitz G. A1

Leitinger G. B4

Leng J.H. I6, O4

Lepensky C. H1, H6, H12

Lévy P. I11

Lewens F. A5

Leyden J. R3, R6, R8, R9

Li J. I6, I19

Li S.C. B6

Li S.S. J5

Li W. E1, J6, L14

Li X.T. I6

Li Y. I4, I6, O4, Q27

Li Z.W. I6

Liang Z.W. I4

Liauw W. L12

Libutti S. L11

Lico V. E8, O9, Q10, Q12

Lim H. B3

Lin L. J5

Linder Ekberg K. Q11

Link E. L12

Lipp R. I8, Q9, R7

Liu J.X. Q26

Liu L.J. J5, J8

Liu L.X. M5

Liu R.R. J5

Lohse A. C6

Loiarro F. A14

Lombard-Bohas C. H15, J3, J12, K9, L3, L10, L16, O13, P6

Lopes J.M. F7

Lopez C. E12

Lopez Escola C. E6

López López C. L7, L8

Losito N. A12

Lou W. J8, M5, O16, R2

Lu C.M. B5, B7

Lugli F. Q8, Q15

Lundedei V. J2

Luo J. Q26

Luong T. B2, O14, R5

Lupp A. A8, E11

Luppi G. J11

Luz R. E9
Maasberg S. J9, L2, L13

Macedo R. Q5

Maddalena P. Q28

Maddalena S. N7

Maiettini D. I7

Maire F. I11

Maisonneuve P. G7

Maisonobe P. K9

Malago' M. O14

Malahias A. I3

Malecka-Panas E. B1

Maletta F. L4

Malfatti V. I9

Malka L. L9

Malleo G. O6

Malpaga A. E2, I9, O5, O6

Mamikunian G. O15

Mandair D. B2

Manfredi R. O5

Mannelli M. A10

Manoharan P. N4

Manon A. K1

Mantovani G. A14

Manzoni M.F. G7

Maragkoudakis E. K10

Marchegiani G. O5

Marciello F. K7, K8

Marconcini R. J10, J11

Marinoni I. A15, G1

Markovich A. K5

Marmissolle F. F6, H3

Marotta V. A12, K7, K8

Marques A.P. F7

Martin J. Q4

Martini C. E8

Mascarenhas L. E9

Massironi S. E7, Q3, Q28

Massoma-Peh P. A13

Masui T. O7

Matsumura Y. Q14

Matteotti D. J4

Maurizi A. R4

Mayerhoefer M.E. J7

Mayo D. O8

Mazzaferro V. G7

McLean J. Q19

McStay M. Q13

Meeker A. H16

Meier-Allard N. C4

Meijer C. A11

Menendez Prieto M.D. E1, L14

Menon K. G8, O10

Merkle E. I15

Merola E. I8, I13

Messerini L. J11

Meyer T. B2, B6, K10, Q24

Meyerson M. B2

Michael M. F4, L12

Michl P. C5 
Milanetto A.C. E8, E12, O9, Q10, Q12

Milione M. G7

Mills L. G8, H14, O10

Miotto M. O5, O6

Mir O. P4

Mita E. Q14

Moati E. L6

Modica R. K7, K8

Modlin I. H1, H5, H6, H12, H13

Mohamed R. I17

Mohmaduvesh M. F9

Moletta L. Q10, Q12

Moll R. A7

Monti M. J2, N6, N7

Moons V. D1

Morgese F. H4

Mori K. Q14

Mormando M. Q15

Morris T. B2

Mortelmans L. I18

Moschouris P. F1, H7

Motta C. F3

Mottaghy F. I18

Mujoomdar A. N5

Mukherjee N. L11

Mulcahy V. Q13

Mullan M. K6

Nackaerts K. I18

Nada H. R5

Naik K. K10

Nakazuru S. Q14

Napolitano V. K8

Narasimha M. K11

Navalkissoor S. L15, Q24

Neumayer B. A9

Nguyen T.X.Q. K1

Niccoli P. K9

Nickerson M. L5

Nicolas G. I15

Nicolini S. N6, N7

Niederle B. B4, J7, R7

Nieveen van Dijkum E, E4

Nieveen van Dijkum E. I5, O3

Nikaki M. I3

Nikolopoulos G. F1

Nishikawa K. Q14

Nishio K. Q14

Noggler P. Q16

Nolan L. E10, O8

Nonaka D. E6

Normand L. A9

Novak K. N5

Nozière C. H15

Nuzzo A. R1

Nyman R. N1

Öberg K. B6, R6

Oboldi D. J2
O’Connor J.M. F6, H3, N2, Q2

Odintsova A. K5

O’Donnell K. M2

Oikonomopoulos N. K10

Oliveira A. L15

Oliveira S. E9

Oneto A. H3

Onofri A. H4

Oosting S. A11

Orel G. K5

Orlova K. G4

Ortolani S. E2, I9, I10

Oudijk L. A10

Owen C. M6

Paganelli G. H5, H6, N6, N7

Page E. N4

Pagliaretta S. H4

Palazzo L. I11

Palazzo M. I11

PALGA: Dutch Pathology Registration, P1

Pandev R. I16

Panis Y. R1

Panzuto F. F5, I8, I13

Papaoikonomou E. F1

Papathomas T. A10

Pape U.F. A7, E3, I12, J9, L2, L13, Q17, Q18

Papotti M. A10, L4

Paradis V. L6

Parisi A. O12

Park J. B3

Park S. B3

Park Y. B3

Partelli S. A3, A4, E12, H4, I8, O11, O12, R4

Pascher A. E3, I12

Pasquali C. B1, E8, E12, O9, Q10, Q12

Pasquer A. O13

Pasquini S. H4

Passegger C. C4, R7

Patila E. Q23

Patyutko Y. D4

Paun D. H9

Pavel M. E3, I2, I12, I14, J9, K2, K3, K4, L2, L10, L13, L16, Q17, Q18, R8

Pavlakis N. F4, I1, L12

Pearce N. E10, O8

Pedrazzoli S. E8, Q12

Peeters M. D1

Pellegrini M.A. F3

Pencharz D. L15

Peracchi M. E7

Peralta O. N2

Pereira B. F7

Perez D. C6

Perez-Casanova L. P5
Perren A. A9, A15, G1

Perros P. K9

Persani L. C2

Persiani R. Q8

Pesce V. F6, H3, N2, Q2

Peters M. A11

Petersen K. A7

Petersen R.H. Q20

Petris R. H9

Pezzilli R. R4

Pezzullo L. A12

Pfragner R. B4, C4, R7, R10

Phan A.T. K2, K3, K4

Piacentini S. Q8, Q15

Pieri F. J2

Piovesan A. L4

Pipinikas C.. B2

Piussi S. F3

Pizza G. K7, K8

Pizzichini P. I8, I13

Planchard D. J12, L9, P4, P6

Plank J. Q16

Plöckinger U. I12

Ploner F. Q16

Poiana C. H9

Polenta V. A3, A4, H4

Polus M. D1

Polycarpou A. K6

Pommier R. L16

Poncet G. O13

Poston G. K9

Pozzi Mucelli R. I10

Prachalias A. G8, O10

Prasad V. E3, H5, I12

Preto J. F7

Price T. F4

Prosperi D. I8, I13

Pschowski R. E3, I2, Q17, Q18

Purinan A. F3

Pusceddu S. G7

Qiao X.W. B5, B7

Quaglia A. G8, H14, O10

Quan D. N5

Quietzsch D. J9, L2, L13

Quigley A.M. L15, Q24

Raderer M. J7, K2, K3, K4

Radice D. J11

Raffaelli M. Q15

Raffenne J. L6

Rahman Z. O14

Ramage J. G8, H2, H14, M2, O10

Ramundo V. A12, K7, K8

Randeva H.S. K11

Rapa I. A10, L4

Rasmussen A.K. Q20

Reardon M.J. O2

Rebonato A. I7
Rebours V. G2

Reddy S. Q5

Regi P. I9

Reid R.H. N5

Reimann C. E11

Reubi J.C. I15

Rezai Kalantari H. D1

Rho Y.S. Q19

Rhotert C. C6

Ribeiro C. E9

Ricci S. J10, J11

Richards-Taylor S. E10

Richter S. N5

Rinaldi S. H4

Rindi G. K2, K3, K4, Q8

Ringholm L. Q20

Rinke A. C5, I8, J9, L2, L13, O11

Rinner B. B4

Rinzivillo M. F5, I8, I13

Riopelle J. O15

Riva E. A6, J4

Riva N. J2

Rizzato C. B1

Roach P. I1

Roberts D. I17

Robev B. I16, Q21

Robledo M. A10

Roca E. F6, H3, N2, Q2

Roche C. A13, L3

Rodrigues F. F7

Rodriguez A. L14

Rodriguez D. E1, J6, L14

Rodriguez-Laval V. I14

Rontogianni D. Q23

Röpke T. I2

Rosales C. Q2

Rosanelli G. Q16

Rosłonowska E. H10

Ross P. G8, O10

Rossi R.E. E7

Rottenburger C. I15

Rudis E. O2

Ruiz A.L. E1, J6, L14

Ruszniewski P. G2, I11, K9, L6, L10, R1, R9

Ruszniewski P.B. K2, K3, K4

Ruzzenente A. I9, I10

Sadjak A. R7

Sakakibara Y. Q14

Salazar R. L10, L11

Salgarello M. I9

Sanatani M. N5

Sanches A.C. F7

Sanchez M.L. E1, J6, L14

Sands A. M4

Sandström M. N1

Sandvik O. D6

Sänger J. A8, E11 
Sansovini M. N6

Santoro A. P4

Santos A.P. F7

Santos D. F6

Sapino A. L4

Saribegioglou N. I3

Sarker D. G8, O10

Sarnelli A. N6

Sato A. O7

Sauvanet A. L6, R1

Sbidian E. L6

Sbrana A. J10

Scagliotti G. L4

Scagliotti G.V. Q1

Scala S. A12

Scaldaferri M. L4

Scaroni C. Q10

Scarpa A. B1, E2, I9, I10, O6

Scheibenbogen C. H11

Schembri G. I1

Schillaci O. I13

Schmidt L. E11

Schmitt A. G1

Schoefberger W. R10

Schrader J. C6

Schulz S. A8, E11

Schwach G. B4, R7, R10

Scialpi M. I7

Sciammarella C. A12

Scoazec J.Y. A13, H15, J3, J12, L3, L9, O13, P4, P6

Scopinaro F. I13

Sedláčková E. K2, K3, K4

Semedo M. E9

Sergieva S. I16, Q21

Severi S. H5, H6

Shapira O.M. O2

Sharma D. O14

Shatwell W. K11

Sheng W. Q7

Sherriff D. Q22

Sherwood R. H2

Shi H. B6

Shi S.S. I4

Siegmund B. A5

Siesling S. D7

Simons S. G3

Singhal N. F4

Sipos B. A7, A8, A9

Sissons M. D2, R3, R6, R8, R9

Sizer B. Q13

Skrobek-Engel G. L2, L13

Skupin J. H8

Smid M. A10

Smirnova E. G4

Smith D. K9

Snead D. K11

Snowdon G. I1

Soli M.P. E1

Solis Hernandez M.P. L14
Solís M.P. J6, L7, L8

Sonetto C. Q1

Song Y.L. B5, B7

Søreide J.A. D6

Søreide K. D6

Sousa M. E9

Spada A. A14

Spada F. F5, G7, J11

Spaggiari P. G7

Specht E. E11

Speel E.J. P1, P2

Speicher M.R. B4

Sperveslage J. A7

Spindelböck W. Q9, R7

Srinivasan P. G8, O10

Srirajaskanthan R. D2, G8, H2, $\mathrm{H} 14, \mathrm{O} 10$

Stangl K. Q17, Q18

Stangl V. Q17, Q18

Stan-Iuga R. R1

Stathopoulos C. Q23

Stavrinidis S. I3

Steadman B. O8

Stefano S. N6, N7

Stepan V. Q16

Stevens M. O15

Stewart G. M6

Strobl H. B4

Strosberg J. G3, H16, L5

Sulovski J. L11

Sun M.F. J5

Sun Y.S. F8, I6

Sundram F. O8

Suppan C. Q9

Svrcek M. L6

Taban S. G5

Taco M.D.R. P5

Tafuto S. A12

Tagliati F. J4

Takaori K. O7

Takhar A. O8

Tam Amersdorfer C. B4

Tamburrino D. E12, O11, O14

Tampellini M. Q1

Tan H.Y. Q26

Tanaskovic N. K6

Tang L. B5, B7, I6

Tartaglia A. J2

Tartaglione L. Q15

Tatangelo F. A12

Tavorath R. L11

Teasdale I. R10

Tegos T. Q23

Teixeira N. H1, H12

Terzea D. H9

Tharmalingam H. M6

Theodoropoulos L. I3

Thiis-Evensen E. D3
Thirlwell C. B2, O14

Thorns C. O1

Thunnissen E. P1

Tilley C. E10

Tipping J. N4

Tischer E. I14

Tomassetti P. F5, L16

Tomkins C. K11

Tonutti L. F3

Tortora G. I9, I10

Toumpanakis C. B2, E12, F9, K6, K10, L15, M6, O14, Q24, Q25

Träger T. A8

Trama A. D7

Trapella C. J4

Trentin C. I10

Tretli S. D3

Trifanescu R. H9

Trimarchi F. K7

Trocóniz I.F. K1

Tsamakidis K. I3

Tschan M. A15

Tsolakis A. I17

Tsoli M. F1

Tumpanakis C. R5

Tzioufa V. G6

Ueda K. R11

Uemoto S. O7

Unger N. J9

Uriol E. E1, L14

Vaidya A. Q5

Valente R. E12, O11, O14

Valle J. E6, K9, L10, M4

Vallerio P. I9, I10

Valloncelli C. I7

Valone T. L5

Van Adrichem R. M3

Van Asselt S. A11

Van Binnebeek S. I18

Van Cutsem E. D1, L10, L16, I18

Van der Zwan J.M. D7

Van Deurzen C. M3

Van Eeden S. E4

Van Eijck C. E4, O3

Van Faassen M. A11, M1

Van Gulik T. O3

Van Hazel G. F4

Van Kemenade F. E4

Van Koetsveld P.M. B8

Van Laethem J.L. D1

Van Suylen R.J. P1

Vanbilloen B. I18

Vanoli A. G7

Varcada M. R5

Vasile E. J10

Vaslamatzis M. Q23
Veenstra M.J. B8

Velidaki A. H7

Venook A. L5

Verbruggen A. I18

Vercherat C. A13, L3

Verheij J. E4

Verslype C. D1, I18

Vescini F. F3

Vianna R. Q5

Vidal A. P5

Vieitez J.M. E1, J6, L14

Vitale G. B8, C2

Vitali E. A14

Vivian G. G8, O10

Vlontzou E. Q23

Volante M. A10, L4, Q1

Vrakas G. Q5

Wald O. O2

Walenkamp A. A11, M1

Walker M. K10, L15, M6

Wall L. K2, K3, K4

Walter T. A13, H15, J3, J12, L3, O13, P6

Wang J. F8

Wang M. Q26

Wang X. F8

Wang Y. J5

Wang Y.H. B5

Wang Y.Z. O15

Wasan H. Q4, Q5

Weber T. L5

Wehweck L. G1

Wei M. O4

Weickert M.O. K11

Wellner U. O1

Wheeler D. M4

White A. K11

Wiedenmann B. E3, I14, Q17, Q18

Wiedmer T. A15

Wild D. I15

Wilfert S. R10

Willowson K. I1

Winkler E.C. J1, L1

Wirtz R.M. E11

Wolin E.M. K2, K3, K4, L11

Woltering E. O15

Wyld D. L12, N3

Xenocostas A. N5

Xiao Y. B5

Xiaokun G. B9

Xin B. J8, M5, O16

Xinopoulos D. I3

Xu J.M. J5

Xu X. M5, O16

Yalchin M. F9, L15, M6, Q24, Q25 
Yamada T. Q14

Yan Z. J8

Yang Z. I19

Yao J.C. L10, L11, L16, R9

Yeend S. F4

Yoo K. B3
Yu J. I19

Yu L.L. Q26

Yu R. B5, B7

Zaccaroni A. J2

Zalcberg J. F4

Zambrowicz B. M4
Zatelli M.C. A2, A3, A4, A6, $\mathrm{C} 1, \mathrm{C} 3, \mathrm{~J} 4$

Zeitlhofer P. B4

Zerbi A. A14

Zgliczyński W. H10

Zhang C. Q26

Zhang M. I17
Zhang Y. J5

Zhao C.H. J5, Q27

Zheng Q. O4

Zhou J. O16

Zhu L.M. B5

Zilli A. E7, Q3, Q28 